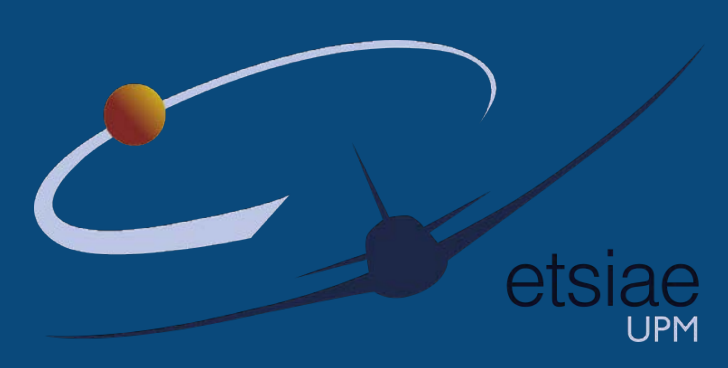

\title{
Efficient Space and Time Solution Techniques for High-Order Discontinuous Galerkin Discretizations of the 3D Compressible Navier-Stokes Equations
}

Doctoral Thesis

By

Andrés Mauricio Rueda-Ramírez

Mechanical Engineer 

Departamento de Matemática Aplicada a la Ingeniería Aeroespacial Escuela Técnica Superior de Ingeniería Aeronáutica y del Espacio

\section{Efficient Space and Time Solution Techniques for High-Order Discontinuous Galerkin Discretizations of the 3D Compressible Navier-Stokes Equations}

by

Andrés Mauricio Rueda-Ramírez

Mechanical Engineer

Advisors:

Eusebio Valero Sánchez

Ph.D. in Aeronautical Engineering

Esteban Ferrer Vaccarezza

Ph.D. in Engineering

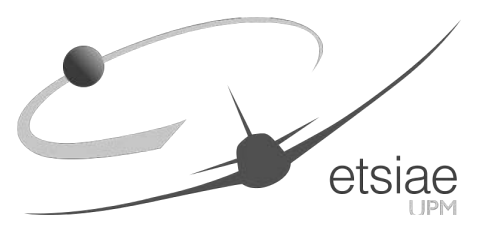

Madrid, September 2019 
This thesis has received funding from the European Union's Horizon 2020 Research and Innovation Program under the Marie Skłodowska-Curie grant agreement No 675008 .

Esta tesis ha recibido financiación del programa Horizon 2020 de investigación e innovavión de la Unión Europea bajo el acuerdo de beca número 675008 del programa Marie Skłodowska-Curie. 


\section{POLITÉCNICA}

El tribunal nombrado por el Sr. Rector Magnífico de la Universidad Politécnica de Madrid, el día de de $20 \ldots . .$.

Presidente: Dr. Javier de Vicente Buendía

Vocal: Dr. David A. Kopriva

Vocal: Dra. Sonia Fernández-Méndez

Vocal: Dr. Oriol Lehmkuhl

Secretario: Dr. Gonzalo Rubio Calzado

Suplente: Dr. François Fraysse

Suplente: Dr. Antonio Pascau

Realizado el acto de defensa y lectura de la Tesis el día 25 de octubre de 2019 en la Escuela Técnica Superior de Ingeniería Aeronáutica y del Espacio (ETSIAE-UPM).

Calificación.

EL PRESIDENTE

LOS VOCALES

EL SECRETARIO 

A mi abuelita, la Machita,

$y$, por supuesto, a Tati. 



\title{
Efficient Space and Time Solution Techniques for High-Order Discontinuous Galerkin Discretizations of the 3D Compressible Navier-Stokes Equations
}

\begin{abstract}
In this thesis, we develop space and time solution techniques for high-order Discontinuous Galerkin (DG) methods. Even though the main focus is the accurate and computationally efficient solution of the 3D compressible Navier-Stokes equations using the Discontinuous Galerkin Spectral Element Method (DGSEM), the methods developed here can be applied to other systems of nonlinear conservation laws and other DG methods. The solution techniques can be classified into two groups: (i) improved solution techniques for temporal discretizations, and (ii) local $p$-adaptation techniques that enhance the spatial discretization.

The first group of techniques decreases the computational time for a given spatial discretization with a fixed number of degrees of freedom (NDOF) by improving the linear and nonlinear solver algorithms. In that regard, special attention is paid to the development of efficient implicit time-integration solvers and to multigrid methods (both explicit and implicit in time). We derive a methodology to obtain the analytical Jacobian of DG discretizations of nonlinear advectiondiffusion equations. Furthermore, we show that time-implicit DGSEM discretizations on Gauss-Lobatto points can be formulated as Schur complement problems and solved using the static-condensation method. This enables one to solve smaller, better-conditioned problems, which yields shorter computation times for moderate polynomial orders.

The second group of techniques modifies the spatial discretization by reducing the NDOF while maintaining similar accuracy. To do that, we develop a novel truncation error estimator that uses the $\tau$-estimation method for the $p$-anisotropic DGSEM. The new method is more accurate and computationally cheaper than previous approaches. The error estimator is then successfully applied to perform local anisotropic $p$-adaptation for steady and unsteady flows. We show that the truncation error estimator can be readily coupled with multigrid techniques to obtain further speed-ups. Moreover, we develop two $p$-adaptation strategies for unsteady flows. One of them is a dynamic $\tau$-based anisotropic $p$-adaptation method. The other one is a static $\tau$-based anisotropic $p$-adaptation method, which may be computationally cheaper in statistically steady flows.
\end{abstract}

Keywords: High-order Discontinuous Galerkin, Implicit time-integration, Staticcondensation, Error estimation, Anisotropic $p$-adaptation, Compressible flows. 



\section{Efficient Space and Time Solution Techniques for High-Order Discontinuous Galerkin Discretizations of the 3D Compressible Navier-Stokes Equations}

\section{Resumen}

En esta tesis se presentan varias técnicas de solución para métodos de Galerkin discontinuo (DG, p.s.s.e.i. ${ }^{1}$ ) de alto orden. Si bien el objetivo principal es el desarrollo de técnicas precisas y computacionalmente eficientes para resolver las ecuaciones de Navier-Stokes de flujo compresible en 3D por medio del método Discontinuous Galerkin Spectral Element Method (DGSEM), todos los métodos desarrollados en esta tesis se pueden aplicar a otras ecuaciones de conservación no lineales y a otros esquemas DG. Las técnicas de solución acá presentadas pueden ser clasificadas en dos grupos: (i) las que están orientadas a acelarar la integración temporal, y (ii) las que mejoran la discretización espacial con adaptación-p local.

El primer grupo de técnicas disminuye el tiempo de cálculo dada una discretización espacial con un número fijo de grados de libertad (NDOF, p.s.s.e.i.) mediante la mejora de los algoritmos de solución. Por ese motivo, se presta especial atención al desarrollo de algoritmos eficientes para integración temporal implícita y de métodos multimalla. También se detalla una metodología para obtener el Jacobiano analítico DG para ecuaciónes de advección-difusión. Además, se demuestra que la discretización implícita del DGSEM en nodos de Gauss-Lobatto puede ser resuelta eficientemente mediante el método static condensation. Lo anterior permite resolver el problema con sistemas lineales más pequeños y mejor condicionados, lo cual disminuye los tiempos de cálculo para órdenes moderados.

El segundo grupo de técnicas consiste en modificar localmente la discretización espacial para reducir NDOF sin deteriorar la precisión del método. Para lograr este obetivo, en esta tesis se desarrolla un nuevo estimador de error para el método DGSEM $p$-anisitrópico basado en el método $\tau$-estimation, el cual es más preciso y requiere menos recursos computacionales que enfoques previos. Posteriormente, este estimador se usa con éxito para realizar adaptación-p anisotrópica en casos estacionaros y no estacionarios, y se demuestra que éste se puede acoplar con métodos multimalla para acelerar más las simulaciones. Adicionalmente, se desarrollan dos técnicas de adaptación- $p$ para casos no estacionarios: un algoritmo dinámico y un algoritmo estático, que es computacionalmente más eficiente en algunos casos estadísticamente estacionarios.

Keywords: Galerkin discontinuo de alto orden, Integración temporal implícita, Static condensation, Estimación de error, Adaptación- $p$ anisotrópica, Flujo compresible.

\footnotetext{
${ }^{1}$ P.s.s.e.i.: por sus siglas en inglés.
} 



\section{Acknowledgements}

The development of this thesis would not have been possible without the support and help of many people who accompanied me during these years.

First of all, I would like to thank my two thesis advisors, Eusebio Valero and Esteban Ferrer, for the immense patience and dedication they have shown during these three years. I thank Eusebio for the trust he placed in me from the beginning, when he hired me in his research group, for always providing me with the necessary means to carry out my research, and for his advice in difficult times. I thank Esteban for being aware of my progress at all times, for being always willing to talk about research, and other topics, and for being ready to advise me what to do when the results were not as expected.

I would also like to thank Gonzalo Rubio for spending so much time explaining his work to me, for helping me find bugs in the code and, in general, for helping me structure my ideas.

I do not have enough words to thank David Kopriva, both for kindly receiving me in San Diego, as well as for the detailed comments and recommendations he gave me during the preparation of this document. David's book and codes were the starting point of my doctoral research, and thanks to him I have been able to find future opportunities. Thank you.

I also thank Vincent Couaillier, Marta de la Llave and Fabio Naddei for hosting me in Paris and for sharing with me their knowledge of the simulation of turbulent flows.

To all the people of the Department of Applied Mathematics in Aerospace Engineering, you have my sincere gratitude. Tere, Alex, Yinzhu, Binghua, Jesus, Juan, Carlos, Diego, Miguel, Marta, Mariola, Vicky, Ignacio, Javi, José, Mario, Bartolo, Jorge, Iván, Fer, you have created an ideal work environment. Thanks to all of you I have managed to grow a lot, not only professionally, but also personally. I am also very grateful to Patricia Alcanda, María Jesús, Inga Dreville, and Carmen Gascó, who have supported me from the administrative point of view.

I want to thank my family, without which this long road would have been impossible. I thank my parents for their unconditional support long before the start of my Ph.D. studies, and for believing in me from the beginning. I also thank my sisters, Tami and Oncia, who have always inspired my love for knowledge, have always been there to listen to me, and have given me their own love.

I also thank my new and old friends. I am really lucky to have you. I especially thank Julio, who despite the distance was always willing to support me and listen to me; Matías, with whom I shared good times in the ghetto; Alex, for the good times in Paris, for his good advice and for sharing with me different points of view, always appreciated; Yinzhu, for sharing a lot with me, from the trips to see auroras, to the construction of model planes; and Jesús, for his kindness, the chocolates and his critical views. 
I am eternally grateful to Tati, who was by my side in good and bad times during these years, who spent sleepless nights just accompanying me to work on my research, and who shared my sorrows and my glories. Without your unconditional support, understanding and infinite love, I would have never been able to complete this process successfully. I will try to give you back the weekends, evenings, nights and vacations that we have stopped enjoying together because of this thesis.

Finally, I would like to thank the Universidad Politécnica de Madrid (UPM) for hosting me during these three years, the Supercomputing and Visualization Center of Madrid (CeSViMa) for providing me with computing resources, and the European Commission for funding this work with the Marie Skłodowska-Curie grant No 675008. 


\section{Agradecimientos}

El desarrollo de esta tesis no habría sido posible sin el apoyo y la ayuda de muchas personas que me acompañaron durante estos años.

En primer lugar, me gustaría agradecer a mis dos directores de tesis, Eusebio Valero y Esteban Ferrer, por la inmensa paciencia y dedicación que han mostrado durante estos tres años. A Eusebio le agradezco la confianza que depositó en mí desde el comienzo al traerme a su grupo de investigación, la diligencia con la que procuró que yo siempre tuviera todos los medios necesarios para realizar mi investigación y sus consejos en momentos difíciles. A Esteban le agradezco por estar pendiente de mis avances en todo momento, por permanecer siempre dispuesto a hablar conmigo sobre temas de investigación, y otros, y por estar presto a aconsejarme qué hacer cuando los resultados no eran los esperados.

Me gustaría agradecer también a Gonzalo Rubio por dedicarle tanto tiempo a explicarme su trabajo, por ayudarme a encontrar errores en el código y, en general, por ayudarme a estructurar mis ideas.

No tengo palabras suficientes para agradecer a David Kopriva, tanto por recibirme amablemente en San Diego, como también por los detallados comentarios y recomendaciones que me dio durante la preparación de este documento. El libro y los códigos de David fueron el punto de partida de mi investigación doctoral, y gracias a él he podido encontrar oportunidades futuras. Gracias.

Agradezco también a Vincent Couaillier, Marta de la Llave y Fabio Naddei por acogerme en París y por compartir conmigo su conocimiento en la simulación de flujos turbulentos.

A todas las personas del Departamento de Matemática Aplicada a la Ingeniería Aeroespacial tengo mucho que agradecerles. Tere, Álex, Yinzhu, Binghua, Jesús, Juan, Carlos, Diego, Miguel, Marta, Mariola, Vicky, Ignacio, Javi, José, Mario, Bartolo, Jorge, Iván, Fer, ustedes han creado un espacio ideal para trabajar. Gracias a todos ustedes he logrado crecer mucho, no sólo en lo profesional, sino también en lo personal. Asimismo, estoy muy agradecido con Patricia Alcanda, María Jesús, Inga Dreville y Carmen Gascó, que desde lo administrativo me han apoyado en este camino.

Quiero dar mil gracias a mi familia, sin la cual este largo camino habría sido imposible. A mis padres por su apoyo incondicional, desde mucho antes de comenzar el doctorado, y por creer en mí desde el comienzo. A mis hermanas, Oncia y Tami, que siempre me han inspirado amor por el conocimiento, siempre me han escuchado cuando las necesito, y me han dado su cariño.

También agradezco inmensamente a mis amigos, los de antes y los nuevos. Soy muy afortunado de tenerlos. En especial les agradezco a Julio, que a pesar de la distancia siempre estuvo dispuesto a apoyarme y escucharme; a Matías, con el que compartí buenos momentos en el ghetto; a Álex, por los buenos momentos en París, sus buenos consejos y por compartir conmigo distintos puntos de vista, siempre 
apreciados; a Yinzhu, por compartir mucho conmigo, desde los viajes a ver auroras, hasta la construcción de aeromodelos; y a Jesús, por su amabilidad, los chocolates y su visión crítica.

Estoy eternamente agradecido con Tati, que estuvo a mi lado en buenos y malos momentos durante estos años, que trasnochó conmigo mientras yo trabajaba, y compartió mis penas y mis glorias. Sin tu apoyo incondicional, comprensión y cariño infinito, yo jamás habría logrado culminar este proceso con éxito. Intentaré devolverte los fines de semana, las tardes, las noches y las vacaciones que hemos dejado de disfrutar juntos por esta tesis.

Por último, quiero agradecer a la Universidad Politécnica de Madrid por acogerme durante estos tres años, al Centro de Supercomputación y Visualización de Madrid (CeSViMa) por proveerme recursos de cálculo, y a la Comisión Europea por financiar este trabajo con la beca número 675008 del programa Marie SkłodowskaCurie. 


\section{Contents}

$\begin{array}{ll}\text { Contents } & \text { XV }\end{array}$

Nomenclature $\quad$ xix

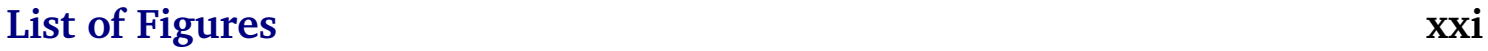

$\begin{array}{ll}\text { List of Tables } & \text { xxv }\end{array}$

1 Introduction 1

1.1 Motivation ......................... 1

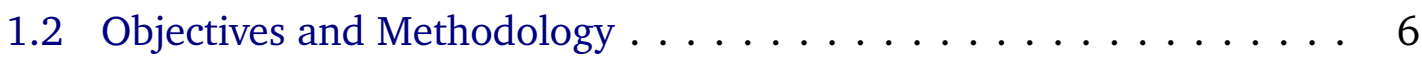

1.3 Outline ........................ 7

1.4 Highlights and Novel Contributions . . . . . . . . . . . . . 8

1.5 Notation . . . . . . . . . . . . . . . . . . . . . 11

I Background: Discretization Methods 17

2 Spatial Discretization $\quad 19$

2.1 The Discontinuous Galerkin Discretization . . . . . . . . . . . . . . 19

2.2 The Discontinuous Galerkin Spectral Element Method (DGSEM) . . . 25

3 Temporal Discretization $\quad 29$

3.1 General Properties . . . . . . . . . . . . . . . . . . . . 30

3.2 Explicit Time-Integration . . . . . . . . . . . . . . . . . . 32

3.3 Implicit Time-Integration . . . . . . . . . . . . . . . . 35

II Solver Technologies for Time-Marching Schemes 37

4 Background: Solution Techniques for Systems of Equations 39

4.1 Introduction . . . . . . . . . . . . . . . . . . 39

4.2 Solving Systems of Nonlinear Equations . . . . . . . . . . . . . . . . . . . . . 40

4.3 Solving Systems of Linear Equations . . . . . . . . . . . . . . . . . . 46 
5 Computing the Jacobian Matrix $\mathbf{5 5}$

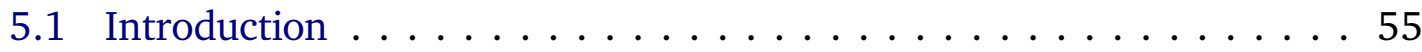

5.2 Numerical Jacobian and Optimizations . . . . . . . . . . . . . . . . . . . . . . . 67

5.3 Jacobian-Free Methods . . . . . . . . . . . . . . . . . . . . . . 60

5.4 Analytical Jacobian for DG Methods . . . . . . . . . . . . . . . . . . 61

5.5 Performance Comparison of the Jacobian Computation Methods . . . 70

5.6 Final Remarks . . . . . . . . . . . . . . . . . . . . . . 71

6 A Static-Condensation Method for the GL-DGSEM 73

6.1 Introduction . . . . . . . . . . . . . . . . 73

6.2 Background: The Static-Condensation Method . . . . . . . . . . . 76

6.3 Jacobian Sparsity Patterns for the DGSEM . . . . . . . . . . . . . . 79

6.4 Static-Condensation for the GL-DGSEM . . . . . . . . . . . . . . . . 85

6.5 Numerical Example . . . . . . . . . . . . . . . . . . . . . . . 87

6.6 Final Remarks . . . . . . . . . . . . . . . . . . . . 93

\section{Enhancing the Spatial Discretization: p-Adaptation 95}

7 Background: On $p$-Adaptation and Error Estimation 97

7.1 Introduction . . . . . . . . . . . . . . . . . 97

7.2 Background on Error Estimation and $p$-Adaptation . . . . . . . . . . 98

7.3 Definition of Errors . . . . . . . . . . . . . . . . . . . . . 102

$7.4 \tau$-Estimation Method . . . . . . . . . . . . . . . . . . . 108

7.5 Nonconforming Discretizations and Mortar Spaces . . . . . . . . . . . 110

8 Truncation Error Estimation in the $p$-Anisotropic DGSEM 113

8.1 Introduction . . . . . . . . . . . . . . . . . . 114

8.2 Low-Order Extrapolation . . . . . . . . . . . . . . . . . . . . 115

8.3 A New Anisotropic Truncation Error Estimator . . . . . . . . . . . . . 118

8.4 Validation of the Anisotropic $\tau$-Estimation Method . . . . . . . . . . . 129

8.5 Final Remarks . . . . . . . . . . . . . . . . . . . . . . . . . 141

9 Steady $p$-Adaptation Using Truncation Error Estimates 143

9.1 Introduction . . . . . . . . . . . . . . . . . . . . . . . . . . . . 143

9.2 Coupling the $p$-Anisotropic $\tau$-Estimation with Multigrid . . . . . . 144

9.3 The $p$-Adaptation Algorithm: Computing the New Polynomial Orders 148

9.4 Multi-Stage Adaptation Process . . . . . . . . . . . . . . . . . . . . 149

9.5 Numerical Results . . . . . . . . . . . . . . . . . . . . . . . . . 150

9.6 Final Remarks . . . . . . . . . . . . . . . . . . . . . . 164 
10 Unsteady $p$-Adaptation Using Truncation Error Estimates 167

10.1 Introduction . . . . . . . . . . . . . . . . . . . 167

10.2 Formulation of the Truncation Error . . . . . . . . . . . . . . . . . 169

10.3 Truncation Error Estimation in Unsteady Problems . . . . . . . . . . . 172

10.4 -Adaptation Strategies . . . . . . . . . . . . . . . . . 173

10.5 Numerical Results . . . . . . . . . . . . . . . . . . . . 178

10.6 Final Remarks . . . . . . . . . . . . . . . . . . . . 186

$\begin{array}{lr}\text { Conclusions } & 189\end{array}$

$\begin{array}{ll}\text { Appendices } & 191\end{array}$

A Compressible Navier-Stokes Equations $\quad 193$

A.1 Jacobians . . . . . . . . . . . . . . . . . . . . . . . . . . . 194

B Alternative Forms to Discretize the Viscous Terms 199

B.1 Primitive Gradients Approach . . . . . . . . . . . . . . . . . . . . . . 199

B.2 Whole Flux Approach . . . . . . . . . . . . . . . . . 200

C HORSES3D: A High Order (DG) Spectral Element Solver 203

C.1 Strong Scalability . . . . . . . . . . . . . . . . . . . . . 204

$\begin{array}{ll}\text { Bibliography } & 209\end{array}$ 



\section{Nomenclature}

\begin{tabular}{|c|c|c|c|}
\hline \multicolumn{2}{|l|}{ Acronyms } & \multirow{3}{*}{$\begin{array}{l}\Omega \\
\vec{\xi}\end{array}$} & \multirow{3}{*}{$\begin{array}{l}\text { Physical domain } \\
\text { Reference frame coordi- } \\
\text { nates }[\xi, \eta, \zeta]\end{array}$} \\
\hline DG & Discontinuous Galerkin & & \\
\hline DGSEM & \multirow{2}{*}{$\begin{array}{l}\text { Discontinuous Galerkin } \\
\text { Spectral Element Method }\end{array}$} & & \\
\hline & & $\underline{\tau}$ & Navier-Stokes stress ten- \\
\hline DOF & \multicolumn{2}{|l|}{ Degree of Freedom } & \\
\hline GL-DGSEM & $\begin{array}{l}\text { Discontinuous Galerkin } \\
\text { Spectral Element Method } \\
\text { on Gauss-Lobatto nodes }\end{array}$ & \multirow[t]{2}{*}{$\mathscr{T}^{N}$} & $\begin{array}{l}\text { Vector containing the } \\
\text { truncation error on all } \\
\text { Gauss/Gauss-Lobatto }\end{array}$ \\
\hline GL & Gauss-Lobatto & & points of a discretizatio \\
\hline NDOF & $\begin{array}{l}\text { Number of (spatial) De- } \\
\text { grees of Freedom }\end{array}$ & \multirow[t]{2}{*}{$\tau^{N}$} & Truncation error of a dis- \\
\hline \multirow[t]{2}{*}{ PDE } & \multirow{2}{*}{$\begin{array}{l}\text { Partial Differential Equa- } \\
\text { tion }\end{array}$} & & $\begin{array}{l}\text { crete discretization of o } \\
\text { der } N\end{array}$ \\
\hline & & $\xi$ & $\begin{array}{l}\text { First reference frame co- } \\
\text { ordinate }\end{array}$ \\
\hline$\epsilon^{N}$ & $\begin{array}{l}\text { Discretization error of an } \\
\text { approximation of order } N\end{array}$ & $\zeta$ & $\begin{array}{l}\text { Third reference frame co- } \\
\text { ordinate }\end{array}$ \\
\hline \multirow[t]{3}{*}{$\mathscr{E}^{N}$} & \multirow{3}{*}{$\begin{array}{l}\text { Generic vector containing } \\
\text { the discretization error of } \\
\text { an approximation of or- } \\
\text { der } N \text { on all Gauss/Gauss- } \\
\text { Lobatto nodes }\end{array}$} & \multicolumn{2}{|c|}{ Roman Symbols } \\
\hline & & $d$ & $\begin{array}{l}\text { Number of space dimen- } \\
\text { sions }\end{array}$ \\
\hline & & \multirow[t]{2}{*}{$\mathbf{I}^{N}$} & \multirow{3}{*}{$\begin{array}{l}\text { Discretizing operator that } \\
\text { filters a continuous solu- } \\
\text { tion } \mathbf{q} \text { to obtain a discrete } \\
\text { solution } \mathbf{q}^{N}\end{array}$} \\
\hline$\eta$ & $\begin{array}{l}\text { Second reference frame } \\
\text { coordinate }\end{array}$ & & \\
\hline \multirow[t]{2}{*}{$\boldsymbol{\varepsilon}_{\mathbf{q}}^{N}$} & \multirow{2}{*}{$\begin{array}{l}\text { Interpolation error of a } \\
\text { state function } \mathbf{q} \text { on a dis- } \\
\text { cretization of order } N\end{array}$} & & \\
\hline & & $I^{N}$ & $\begin{array}{l}\text { Sampling operator th } \\
\text { filters a continuous solt }\end{array}$ \\
\hline
\end{tabular}


tion $\mathbf{q}$ to obtain a discrete solution in the whole domain $\mathbf{Q}^{N}$

$\stackrel{\leftrightarrow}{\mathrm{f}} \quad$ Flux tensor

$\stackrel{\leftrightarrow}{\mathbf{f}}^{a} \quad$ Advective flux tensor

$\overleftrightarrow{\mathbf{f}}^{v} \quad$ Diffusive flux tensor

$\underline{\mathbf{I}}_{P}^{N} \quad$ Interpolation (projection) operator of a solution of order $P$ to a solution of order $N$

$\underline{\underline{\underline{G}}} \quad$ Jacobian of the flux with respect to $\vec{\nabla} \mathbf{q}$

A Jacobian matrix

J Jacobian of the flux with respect to $\mathbf{q}$

$K \quad$ Number of elements

$n_{\text {cons }}$

$v_{n}$

$\vec{x}$

$N$ on a normal vector $v_{n}=$
Number of conservative variables

Projection of the velocity $\vec{v} \cdot \vec{n}$

Physical frame coordinates $[x, y, z]$

Polynomial order of the discretization. Depending on the context, it is used to note the exact polynomial order of an Element

$N=\left(N_{1}, N_{2}, N_{3}\right)$ or just to indicate that a solution is discrete, e.g. $\mathbf{q}^{N}$, in which case $N$ can have different values in different elements and directions

$\mathbf{q}$

$\mathbf{q}^{N}$

$\Re$

$\mathfrak{r}$

$\tilde{\mathfrak{R}}$

$\mathbf{u}$

$u$

$\hat{\imath}$

$\hat{\imath}_{i}$

$\hat{\jmath}$

$\hat{k}$

$v$

$\vec{v}$

w

State vector of conserved variables

Discrete solution of order $N$

Residual vector

Residual state vector

Residual vector scaled with mass matrix: $\underline{\mathbf{M}}^{-1} \mathfrak{R}$

State vector of primitive variables

Velocity in the x-direction Unit vector in the $\mathrm{x}$ direction

Unit vector in the $i^{\text {th }}$ direction: $\hat{\imath}_{1}=\hat{\imath}$

Unit vector in the $y$ direction

Unit vector in the $\mathrm{z}$ direction

Velocity in the y-direction

Velocity spatial vector

Velocity in the z-direction 


\section{List of Figures}

2.1 Example of a 1D domain subdivision into finite elements with piecewise solutions. . . . . . . . . . . . . . . . . . . . . 20

2.2 Lagrange interpolating polynomials on Legendre-Gauss and LegendreGauss-Lobatto points. . . . . . . . . . . . . . . . . . 26

4.1 FMG- and V-cycing strategies. Equal signs represent the continuation of the V-cycling process until reaching the desired residual. . . . 45

5.1 A simple $2 \mathrm{D}$ mesh of $3 \times 3$ and its corresponding sparsity pattern. . . 58

5.2 A simple 2D mesh of $6 \times 6$ and its corresponding sparsity pattern. . . 59

5.3 Absolute error of the numerical Jacobian as a function of $\epsilon$ (first order approximation with 64-bit double precision variables). . . . . 60

5.4 Linear system, $\mathbf{A} \Delta \mathbf{Q}=\mathbf{B}$, that results from the time-implicit DG discretization on the simple 2D mesh of Figure 5.1(a). The contribution of a specific degree of freesom is highlighted. . . . . . . . . . . 64

5.5 Sparsity pattern for the mesh of Figure 5.1(a) when using a noncompact viscous numerical flux. . . . . . . . . . . . . . 66

5.6 Sequential Jacobian computation times for the different techniques. . 71

6.1 Meshes analyzed for sparsity patterns showing the element numbering. 79

6.2 Sparsity patterns of system matrices for 1D scalar advective equations. 81

6.3 Sparsity patterns of system matrices for advective 3D single-equation discretizations. . . . . . . . . . . . . . . . . 81

6.4 Sparsity patterns of system matrices for advection-diffusion 1D singleequation discretizations. . . . . . . . . . . . . . . . 83

6.5 Sparsity patterns of system matrices for advection-diffusion 1D singleequation discretizations. . . . . . . . . . . . . . . . . 84

6.6 Detail of the Jacobian blocks corresponding to elements 1 and 2. . . 85

6.7 Matrix condensation operations for the 3D Navier-Stokes $\left(n_{\text {cons }}=5\right)$ case: $\underline{\mathbf{A}}_{b b}-\underline{\mathbf{A}}_{i b} \underline{\mathbf{A}}_{i i}^{-1} \underline{\mathbf{A}}_{b i}=\underline{\mathbf{A}}_{C}$. Every pixel corresponds to a matrix entry. 86 
6.8 Ratio of condensed system size to global system size (3D diffuser example). . . . . . . . . . . . . . . . . . 87

6.9 Cylinder test case. . . . . . . . . . . . . . . . . . . . . 88

6.10 Residual norm vs. CPU-time for the cylinder flow at $\operatorname{Re}_{\infty}=30$. Solid lines represent the purely explicit simulations (RK3), dashed lines are the implicit simulations solved globally (BDF1), and dotted lines are the implicit simulations solved with the static-condensation method (BDF1+static-condensation) . . . . . . . . . . . . 8 89

6.11 Enhanced performance of the static-condensation method for the cylinder test case. . . . . . . . . . . . . . . . . . 90

6.12 L2 condition number of the system matrix for the global system and the statically condensed one. . . . . . . . . . . . . . . 991

6.13 Residual norm vs CPU-time for the cylinder at $\operatorname{Re}_{\infty}=30$. Even polynomial orders. . . . . . . . . . . . . . . . . . . 92

6.14 Performance of the static-condensation method with a GMRES solver. 92

7.1 Representation of the mortar space $\Xi$ that connects the faces L and R.111

8.1 One- and two-dimensional truncation error maps constructed with $P=5$ showing estimated and extrapolated values for a toy problem. 116

8.2 Hyperplane behavior of the truncation error of a toy problem. . . . . . 118

8.3 Spatial representation of two-dimensional anisotropic truncation error maps. . . . . . . . . . . . . . . . . . . . . 127

8.4 Density $(\rho)$ contours for the proposed manufactured solutions test case. . . . . . . . . . . . . . . . . . . . 130

8.5 Truncation error maps for element A (logarithmic scale). . . . . . . . . 131

8.6 Contour of the number of degrees of freedom against the polynomial order. The combinations $\left(N_{1}, N_{2}\right)$ that fulfill the $\tau_{\max }$ threshold are marked with black squares. . . . . . . . . . . . . . . . . 131

8.7 Spatial representation of Two-dimensional anisotropic truncation error maps for the manufactured solutions test case. . . . . . . . . . . 133

8.8 Truncation error maps for element A (logarithmic scale). . . . . . . . . 134

8.9 Truncation error estimation for $N_{2}=4$ in element A (dotted line of Figure 8.8). . . . . . . . . . . . . . . . . . . . 134

8.10 Truncation error estimation for $N_{1}=4$ in element A (dashed line of Figure 8.8). . . . . . . . . . . . . . . . . . . 134

8.11 Exact values of the achieved errors after the anisotropic $p$-adaptation based on non-isolated and isolated truncation error estimators with $N_{\min }=1$ and $N_{\max }=10 \ldots \ldots \ldots \ldots \ldots \ldots \ldots \ldots \ldots \ldots \ldots \ldots \ldots$

8.12 Density $(\rho)$ contours for the proposed manufactured solutions test case with curved boundaries. . . . . . . . . . . . . . 138 
8.13 Anisotropic truncation error estimation (obtained with a reference solution of order $P_{1}=P_{2}=5$ ) under high-order mappings and comparison with the exact truncation error in element A for fixed $N_{1}=4$ (a,c,e,g) and $N_{2}=4$ (b,d,f,h) - logarithmic scale. . . . . . . . . . 139

9.1 Adaptation process: Anisotropic 3V FAS cycle and subsequent adaptation. . . . . . . . . . . . . . . . . . . . . 147

9.2 Proposed FMG cycle with multiple adaptation stages. Equal signs represent the continuation of the V-cycling process until the desired residual is reached. . . . . . . . . . . . . . . . . . . 150

9.3 Flat plate at $\operatorname{Re}_{\infty}=6000$. x-Momentum contour and velocity profiles at different positions, as compared with the exact solution by Blasius. . . . . . . . . . . . . . . . . . . . . . . . 151

9.4 Comparison of the performance of an RK3 method and the isotropic / anisotropic FAS $p$-multigrid method (with RK3 as a smoother) for solving the subsonic boundary layer test problem $(N=10) \ldots \ldots$. . . 152

9.5 Relative error in the drag coefficient calculation for different methods. The reference drag $C_{d}^{P=15}$ was calculated on a uniformly refined mesh with $P=15$. The blue lines represent uniform refinement, the red lines represent the $\hat{\tau}$-based $p$-adaptation procedure with $N_{\max }=10$, and the black lines with $N_{\max }=5$. Overlapping curves in (a). . . . . . . . . . . . . . . . . . . . . . . 154

9.6 Contour indicating the final average polynomial orders after the adaptation procedure (a) and a detail of the Gauss-Points that shows the anisotropic nature of the $p$-adaptation method (b) for a threshold of $\hat{\tau}_{\max }=10^{-3}$, which produces a relative drag error of $e_{\text {drag }}^{N=15}=$ $1.49 \times 10^{-4}$. White boxes represent $N_{1}=N_{2}=1 . N_{a v}=\left(N_{1}+N_{2}\right) / 2 . \quad .155$

9.7 Number of degrees of freedom after adapting the mesh with different thresholds $\left(\hat{\tau}_{\max }\right)$ and different reference meshes $(P)$ for $N_{\max }=20.156$

9.8 Comparison of a single-stage and a multi-stage adaptation process for solving the boundary layer test case with a truncation error threshold of $\hat{\tau}_{\max }=4 \times 10^{-3}: N_{\max }=20$ (a), and $N_{\max }=30$ (b). . . . . . 157

9.9 Sphere at $\operatorname{Re}_{\infty}=200$. x-Momentum contour and pressure coefficient $\left(C_{p}\right)$ as compared with data by Fadlun et al. [59] and the

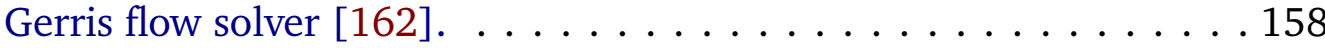

9.10 Relative error in the drag and lift coefficients for the sphere flow problem. The blue lines represent uniform refinement, and the red lines represent the $\hat{\tau}$-based $p$-adaptation procedure with $N_{\max }=7 . . .161$

9.11 Contours indicating the final polynomial orders after $p$-adaptation for the sphere test case: Average polynomial orders $\left(N_{a v}\right)$ (a) and a detail of the Gauss-Points that shows the anisotropic nature of the $p$-adaptation method (b) for a threshold of $\hat{\tau}_{\max }=4 \times 10^{-4}$. White boxes represent $N_{1}=N_{2}=N_{3}=2 . . N_{a v}=\left(N_{1}+N_{2}+N_{3}\right) / 3 . \ldots 163$ 
9.12 Comparison of single-stage $(P=4$ and $P=5$ ) and multi-stage adaptation $\left(\mathscr{P}_{1}=4, \mathscr{P}_{2}=8\right)$ processes for the sphere. $N_{\max }=11$,

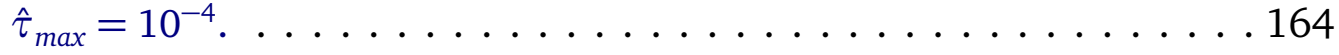

10.1 Flowchart of the dynamic $p$-adaptation. . . . . . . . . . . . . . . . 174

10.2 Flowchart of the static $p$-adaptation. . . . . . . . . . . . . . . 176

10.3 Initial condition of the flowing vortex simulation. . . . . . . . . . . . 179

10.4 Error performance of the static and dynamic $p$-adaptation procedures for a polynomial order jump codition of $N_{i}^{+} \geq\left\lfloor 2 N_{i}^{-} / 3\right\rfloor . \ldots 180$

10.5 Error performance of the static and dynamic $p$-adaptation procedures for a polynomial order jump codition of $N_{i}^{+} \geq\left\lfloor N_{i}^{-}-1\right\rfloor \ldots . .180$

10.6 Average polynomial order distribution for static $p$-adaptation with $\tau_{\max }=10^{-1}$ and different estimation intervals. . . . . . . . . . 182

10.7 Combined effect of the polynomial order jump condition and the estimation interval on the error. . . . . . . . . . . . . . . . 183

10.8 Vortex shedding behind a cylinder at $\operatorname{Re}_{\infty}=100 \ldots \ldots$. . . . . . . . 184

10.9 Comparison of the two possible formulations of the truncation error: traditional (left) and new (right). Average polynomial order distribution for static $p$-adaptation for different error thresholds. . . . 185

10.10Performance of the static and dynamic $p$-adaptation procedures for the flow past a cylinder at $\operatorname{Re}_{\infty}=100, \mathrm{Ma}_{\infty}=0.15 \ldots \ldots \ldots 8$

C.1 Strong OpenMP scalability test. . . . . . . . . . . . . . . . . . . . 205

C.2 Strong scalability solving the TGV problem with a $32^{3}$ mesh. . . . . . 206

C.3 Strong scalability solving the TGV problem with a $64^{3}$ mesh. . . . . . 207 


\section{List of Tables}

1.1 Summary of notation $\ldots \ldots \ldots \ldots \ldots \ldots \ldots \ldots$

3.1 Williamson's low-storage third order Runge-Kutta coefficients . . . . 34

3.2 Coefficients for BDF up to order $5 \ldots \ldots \ldots \ldots \ldots$

6.1 Ratio of condensed system size to global system size . . . . . . . . 86

8.1 Allowable mapping orders for all the discretizations needed in an anisotropic error estimation. $P_{1}=P_{2}=5$ and $M_{1}=M_{2}=4 \ldots \ldots 138$

9.1 Computation times and speed-up for four methods to get a relative drag error of at least $1.8 \times 10^{-4}$ after converging to $\|\mathfrak{R}\|_{\infty}<10^{-9}$. . 154

9.2 Drag coefficient for sphere at $\operatorname{Re}_{\infty}=200 \ldots \ldots \ldots \ldots \ldots \ldots 158$

9.3 Computation times and speed-up to get a drag error of $e_{\text {drag }} \leq 5.31 \times$ $10^{-4}$ (the lowest achieved). All simulations are converged to $\|\mathfrak{R}\|_{\infty}<$

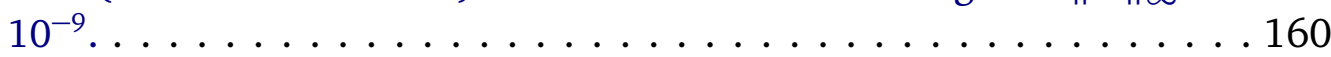

9.4 Computation times and speed-up to get a lift error of $\left|C_{l}\right| \leq 3.34 \times$ $10^{-4}$ (the lowest achieved). All simulations are converged to $\|\mathfrak{R}\|_{\infty}<$ $10^{-9} \ldots \ldots \ldots \ldots \ldots \ldots$. . . . . . . . . . . . . 160

9.5 Summary of performance for single- and multi-stage simulations with $\hat{\tau}_{\max }=10^{-4} \ldots \ldots \ldots \ldots \ldots \ldots \ldots \ldots \ldots \ldots \ldots \ldots \ldots \ldots$

10.1 Possible outcome of the approach 1 to static $p$-adaptation. . . . . . 177 



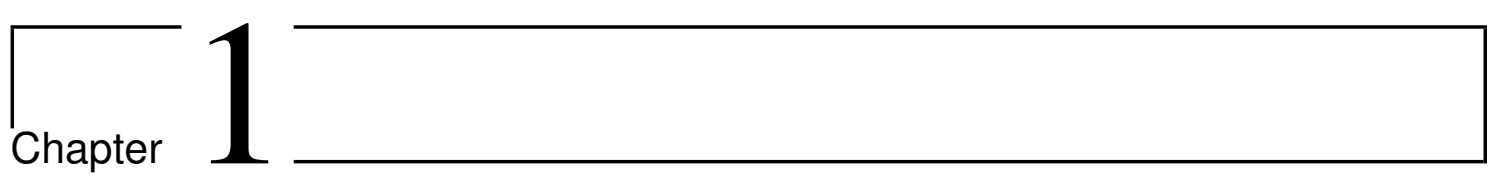

\section{Introduction}

\section{Contents}

1.1 Motivation ................... 1

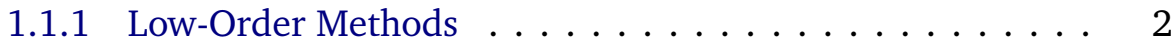

1.1.2 High-Order Methods . . . . . . . . . . . . . 4

1.1.3 Challenges of High-Order DG Methods . . . . . . . . . 5

1.2 Objectives and Methodology . . . . . . . . . . 6

1.3 Outline ..................... 7

1.4 Highlights and Novel Contributions $\ldots \ldots \ldots \ldots$

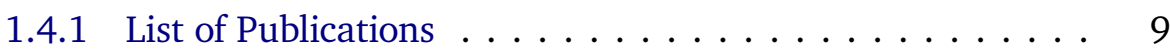

1.5 Notation . . . . . . . . . . . . . . . . . 11

\subsection{Motivation}

The understanding of three-dimensional (3D) flows and the ability to predict their behavior are essential in many industrial applications [5, 40, 183], from the design phase of products to their maintenance and failure analysis; for our comprehension of complex physical phenomena, such as large oceanographic and astrophysical flows [36, 147]; for biological and biomedical engineering in the design of medical devices [105, 164]; for environmental engineering [139], from weather forecasting to the understanding of climate change, adaptation and mitigation strategies; among many other applications.

The physics of fluid flow can be mathematically represented as a system of nonlinear integral or partial differential equations (PDEs), see e.g. Appendix A, which in general does not admit analytical solutions. As a result, fluid mechanics has been historically studied using experimental setups, and numerical methods that approximate the solution of the mathematical models, a technique often known as Computational Fluid Dynamics (CFD). Experimental investigation is still of paramount importance since it provides the most reliable data if properly done; however, it 
may be very expensive or even impossible to conduct. On the other hand, CFD techniques have gained an important place in the study of complex flow problems as a consequence of the constant increase of computational resources and development of algorithms in recent decades [146].

CFD methods reduce the complex nature of a physical problem to a large number of simple arithmetical operations that can be calculated by a computer, while trying to maintain the underlying nature of the original PDEs. Even though the boom of CFD has contributed to models becoming more detailed and complete, there are still several challenges that must be addressed in terms of accuracy and computational performance, and for turbulence modeling [190, 202]. Experimental setups are still commonly used for the verification and validation of numerical techniques. For this reason, there is active research on the development of accurate and computationally efficient simulation tools for 3D flows.

Among the different discretization techniques that have been proposed, we distinguish between low-order and high-order methods. Most numerical methods discretize the physical domain into a grid and represent partial derivatives as functions of the sought-after solution and the local grid spacing $(h)$. As a convention, we consider a numerical scheme to be low order when the error behaves as $\mathscr{O}\left(h^{W}\right)$, where $W<3$, and high order when $W \geq 3$. The traditional finite differences, finite volumes and finite elements are therefore considered low-order methods; whereas spectral methods, high-order continuous and discontinuous Galerkin schemes are inherently high order ${ }^{1}$.

\subsubsection{Low-Order Methods}

The Finite Difference (FD) method is the oldest known approach to approximate the solution of PDEs, as it was originally introduced by Leonhard Euler in the $18^{\text {th }}$ century $[66,99,100]$. The method uses the differential form of the equations and directly discretizes the spatial derivatives as truncated Taylor expansions, so that the solution on every grid point is linked to the one on neighbor points as a function of the grid spacing, $h$. Since the FD method discretizes the differential equations directly without further manipulation, it is said to use the strong form of the equations. This method has been broadly used and validated, and it is the simplest numerical scheme to implement for structured grids ${ }^{2}$.

Theoretically, the FD method can be extended to any order of accuracy by including several terms of the Taylor expansion. However, in practice this increases the region of the domain every degree of freedom depends on (also called stencil), which complicates the imposition of boundary conditions and restricts the method to structured grids. Moreover, FD schemes do not enforce conservation unless special care is taken. Consequently, FD schemes are not the most popular methods in

\footnotetext{
${ }^{1}$ The original discontinuous Galerkin method by Reed and Hill [166] was only of first order.

${ }^{2} \mathrm{~A}$ structured (or regular) grid is a tessellation of an $n$-dimensional Euclidian space by congruent parallelotopes.
} 
computational fluid dynamics today.

The Finite Volume (FV) method is without a doubt the most studied numerical method to solve advection-dominated PDEs, like the ones usually encountered in fluid dynamics [57]. This method, which started to broadly be used in the 1950s, starts with the integral version of the Navier-Stokes equations, divides the physical domain into a finite number of control volumes, and obtains discrete representations of the Navier-Stokes equations that retain their conservative character locally in each volume. The interaction between finite volumes is determined by solving a Riemann problem [193], which ensures an adequate treatment of wave propagation problems (proper upwind conditions). Additionally, the FV method can be used on unstructured grids ${ }^{3}$, a feature that gives it the capability to solve practical industrial problems.

Due to the effort of many years of development, the computational efficiency of FV codes was enormously improved in the second half of the twentieth century using strategies like mesh adaptation techniques [9, 31, 88, 143], implicit timeintegration schemes with very efficient preconditioners [39, 89, 107], multigrid methods [33, 34, 101, 143], etc. FV methods are traditionally first- or secondorder accurate, i.e. $W=1$ or $W=2$. Increasing the order of accuracy requires larger stencil sizes, which hinders the use of unstructured grids and deteriorates the accuracy in the vicinity of boundaries as compared to inner cells.

The Finite Element Method (FEM) is another prominent numerical discretization strategy. It was developed for solid and structural mechanics problems [207], which is still its main field of application. The FEM uses the so called weak formulation: the original PDE being solved is multiplied by an arbitrary test function and the resulting equations are integrated by parts over the simulation domain. This procedure relaxes the smoothness requirements of the solution and, therefore, provides robustness when the coefficients of the equation are discontinuous. The solution and test functions are reconstructed as a linear combination of basis (or Ansatz) functions, which are required to be continuous across element interfaces.

In the 1970's, the Finite Element Method was extended to fluid dynamics problems [100] and was realized that it is not robust when directly applied to advectiondominated flows. To avoid this drawback, the Stream Upwind Petrov-Galerkin (SUPG) method was proposed [102], which relies on the same principles as the FEM, but introduces additional terms to stabilize the numerical solution. Several other strategies have been formulated to provide robustness to the FEM in advection-dominated flows, such as the local projection stabilization (LPS) [29, 30], the edge oriented stabilization (EOS) [195], among others.

The Discontinuous Galerkin (DG) method was initially proposed by Reed and Hill in 1973 [166] for solving the neutron transport equation. The DG approach can be interpreted as a finite element method where the continuity constraint on element interfaces is relaxed, allowing for discontinuities in the numerical solution.

\footnotetext{
${ }^{3}$ An unstructured grid, as opposed to a structured grid, is constructed with geometrical forms that are not parallelotopes, e.g. triangles, tetrahedra, prisms, etc.
} 
This feature makes DG more robust than FEM for describing advection problems when an appropriate Riemann solver is selected [193]. In DG, the finite element weak formulation leads to the appearance of volume and surface integrals that are often evaluated using quadrature rules.

\subsubsection{High-Order Methods}

Spectral methods emerged as an alternative to traditional low-order methods in the 1970s [42] with a very different discretization approach that projects the solution into a high-order space of functions of order $N .{ }^{4}$ This strategy allows one to easily obtain schemes with arbitrary high-orders of accuracy, which translates to low dispersion and dissipation errors for smooth solutions [42, 104]. The result is a reduction in the number of degrees of freedom (NDOF) for a desired accuracy (see for example $[42,62]$ ). Some spectral methods start with strong formulations, whereas some others are derived from weak formulations.

As spectral methods were initially developed as single-domain methods, their main drawback was their inability to represent complex geometries. A remedy has been found with the formulation of multi-domain spectral methods, where a spectral method is formulated inside each element of a mesh. Examples of these methods are the high-order continuous Galerkin [104, 199] and discontinuous Galerkin $[95,148,165,168]$ methods, among others. In this thesis, high-order discontinuous Galerkin methods are used since, they provide very interesting properties that make them well suited for the simulation of fluid dynamics problems.

High-order DG methods are a combination of the traditional DG method and spectral methods that has gained increased popularity in the last two decades. In the beginning of the twenty-first century, high-order DG methods made their way into the mainstream of CFD, when they were proved to be well suited for solving convection-diffusion equations [14, 48, 49]. Commonly, high-order DG schemes are formulated as multi-domain spectral methods. As a result, besides the increased accuracy of spectral (high-order) methods (they can exhibit an optimal order of convergence of $\mathscr{O}\left(h^{N+1}\right)$ ), they also provide a compact stencil and therefore a local character, a feature that makes them highly parallelizable and flexible for complex 3D geometries [48, 97, 202].

Because of the robustness of the DG method to describe advection-dominated problems and the enhanced accuracy of spectral discretizations, high-order DG methods have been widely used to solve the compressible and incompressible NavierStokes equations [49, 63, 97, 202]. Moreover, DG methods have the remarkable property of being able to handle nonconforming meshes with hanging nodes and/or different polynomial orders efficiently, which makes them well suited for mesh adaptation strategies [64, 116, 168].

\footnotetext{
${ }^{4}$ Throughout this thesis, the polynomial order is denoted by $N$, but the action of locally adapting the polynomial order is called $p$-adaptation, as it is commonly called in the literature.
} 
Conventionally, high-order DG methods represent the solution inside every element of the mesh as a linear combination of polynomial basis functions. Two approaches are available for the representation of the polynomial: the modal and the nodal. In the modal approach, the basis functions are typically the different modes of Chebyshev or Legendre polynomials and the unknown quantities are the coefficients that multiply every basis function. In the nodal (or collocation) approach, the unknown quantities are the values of the solution at specific location points of the element, and the solution is reconstructed with an interpolating polynomial (e.g. the Lagrange interpolating polynomials).

Among the different DG formulations, the discontinuous Galerkin spectral element method (DGSEM) $[23,113,116]$ is a nodal (collocation) version of the DG method that uses tensor-product Lagrange basis functions (traditionally in hexahedrals) and stores the data at the Gauss or Gauss-Lobatto nodes of the quadrature rule. The use of a quadrature rule that is the same order as the approximate solution equips the DGSEM with a diagonal mass matrix and very cheap-to-compute operators. In fact, the computational cost of the DGSEM has been estimated to be a factor of four smaller than other conventional DG methods [17].

The advantage provided by the insufficient order quadrature rule comes with a disadvantage: the traditional DGSEM suffers from aliasing errors that can trigger instabilities, specially at high Reynolds numbers [135]. Fortunately, a solution has been found with the use of a split (or skew-symmetric) form of the governing equations $[16,77,80]$, which is possible since it has been shown that the Gauss-Lobatto DGSEM (GL-DGSEM) satisfies the summation-by-parts (SBP) property [77]. The split form has a dealiasing effect which makes the DGSEM provably stable (something that is not achievable with polynomial dealiasing), yielding results comparable to over-integrated quadrature dealiased DG methods [204].

In addition, since the DGSEM uses tensor-product bases, it can handle $p$-anisotropic discretizations efficiently $[110,116,180]$, i.e. discretizations that have different polynomial orders in each coordinate direction. For all those properties, the DGSEM has been used in a wide range of applications, including the simulation of the Maxwell's equations [116], the compressible Euler and Navier-Stokes equations $[16,35,54,71,134,148,165]$, multi-phase flows [71, 136], magnetohydrodynamics flows [26], etc.

Taking into account all these advantages in comparison with other DG methods, the DGSEM is the method used in this thesis.

\subsubsection{Challenges of High-Order DG Methods}

In spite of all the talent and effort invested in making high-order DG methods competitive, still most of the production-quality CFD codes in use are low-order: e.g. in the aerospace industry [5, 40, 183], in weather prediction [139], in astrophysics $[36,147]$, etc. A likely reason is that the the long years of development on loworder methods have produced very computational-cost-effective solvers that are 
difficult to compete against. For instance, the number of publications on preconditioners for FV methods is much larger than for high-order DG.

A recent survey paper by Wang et al. [202] points out that the major challenges that the high-order community must address in order to impact the design process and replace traditional low-order codes are: (i) to improve the high-order mesh generation, (ii) to develop efficient mesh adaptation strategies, (iii) to investigate on cost-efficient time integrators, and (iv) to advance on robust shock-capturing techniques.

This thesis focuses on two of those major topics: the development of computationally efficient time integrators and mesh adaptation strategies for high-order methods, and the application of them to 3D compressible flows.

\subsection{Objectives and Methodology}

This thesis develops computationally efficient solution techniques for arbitrary highorder Discontinuous Galerkin methods and applies them to compressible flow problems in three space dimensions. This objective is achieved by working on two main fronts: the temporal discretization and the spatial discretization.

First, regarding the temporal discretization, this thesis provides efficient methods that allow one to reduce the computational cost for a specific spatial discretization with a fixed number of degrees of freedom. Particularly, we implement and improve implicit time-integration schemes and nonlinear multigrid algorithms for steady and unsteady flows. Traditionally, high-order methods have employed explicit time-marching schemes. These methods have an inherent stability constraint that forces the time-step to be reduced when the mesh size is small. An important feature of fluid flows is the presence of boundary layers near walls which must be captured with refined meshes (or high polynomial orders). As a consequence, the time-step in an explicit simulation is generally bounded by the stability constraint and not by the accuracy of the method.

Part of this thesis is dedicated to the efficient computation of the Jacobian matrix and to the development of efficient solvers for time-implicit DG methods. Implicit time-integration schemes are attractive alternatives since they are usually not restricted by a stability constraint limiting the time-step size. Therefore, the choice of the time-step is mostly dependent on the desired accuracy. The disadvantage of implicit methods is that they require one to solve several systems of nonlinear equations. These systems may be difficult to solve, and the parallel implementation is not straightforward as in explicit schemes. Furthermore, in many cases, it is necessary to obtain the Jacobian matrix of the discretization, a task that is storageintensive and may be hard to do in an efficient manner [12].

Multigrid solvers can be used to solve linear and nonlinear systems of equations and they can be employed to accelerate the solution of explicit and implicit timeintegration schemes. Multigrid has been extensively used with low-order methods, 
such as traditional Finite Difference and Finite Volume schemes [101, 123, 198]. More recently, multigrid methods have also been used to accelerate high-order DG time marching computations for a fixed number of degrees of freedom with great success $[13,28,68,131,132,149,151,185,200,201]$. In this thesis, we focus on the analysis of the nonlinear multigrid scheme and its capacity to enhance the performance of DGSEM implementations as a solver and, as will be shown in this thesis, as an error estimator for mesh adaptation strategies.

With regard to the spatial discretization techniques, this thesis provides efficient strategies to perform local mesh adaptation for 3D compressible flows. The idea behind these strategies is to reduce the number of degrees of freedom (DOFs) while maintaining high accuracy, which translates into shorter computational times and reduced storage requirements. As was discussed previously, DG methods are well suited for mesh adaptation strategies since they are able to handle nonconforming meshes with hanging nodes and/or different polynomial orders efficiently $[64,116,168]$.

Local adaptation can be performed by subdividing or merging elements (hadaptation), by enriching or reducing the polynomial order in certain elements ( $p$-adaptation), by relocating the position of the nodes in a mesh (r-adaptation), or by a combination. For all these strategies it is of paramount importance to identify the flow regions that require refinement or coarsening with a local error estimation.

Part of this thesis is devoted to the formulation of computationally efficient and accurate truncation error estimators and their use for $p$-adaptivity in steady and unsteady flow simulations. We focus on truncation error estimates since it has been shown that a reduction of the truncation error controls the numerical accuracy of all functionals [110], hence reducing the truncation error leads to a more accurate simulation in general.

\subsection{Outline}

The remaining chapters of this thesis are divided into three parts:

- Part I provides a general overview of the discretization methods that are used throughout the document. First, Chapter 2 details the spatial discretization strategy: the formulation of the arbitrary high-order Discontinuous Galerkin Spectral Element Method for three-dimensional nonlinear problems is presented, and its application to complex geometries using a high-order curvilinear mapping is explained. Chapter 3 addresses the temporal discretization: a survey of time-marching schemes is presented, and the accuracy and cost of the different methods is discussed.

- Part II is devoted to the enhancement of the computational efficiency of the solver technologies that are needed for different time-marching schemes. Chapter 4 presents a compilation of solvers for linear and nonlinear systems 
of equations that are used for implicit and explicit time-integration methods. In Chapter 5, we analyze different strategies to compute the Jacobian matrix of a time-implicit DGSEM, where the formulation and implementation of the DG analytical Jacobian is detailed. Additionally, a novel static-condensation method for the Gauss-Lobatto DGSEM is presented in Chapter 6.

- Part III deals with the improvement of the efficiency of the spatial discretization through local $p$-adaptivity, i.e. the reduction of the computational cost by reducing the NDOFs in a smart way. Chapter 7 provides the machinery needed to perform $p$-adaptation: mortar spaces for $p$-nonconforming DGSEM discretizations, definition of error measures and the $\tau$-estimation method. In Chapter 8, we formulate and validate a novel, accurate and low-cost truncation error estimator for the $p$-anisotropic DGSEM. In the rest of this part of the thesis, this truncation error estimator is used to develop several $p$-adaptation strategies for steady (Chapter 9) and unsteady (Chapter 10) flow problems.

Finally, the main conclusions and prospects are summarized in the chapter Conclusions.

\subsection{Highlights and Novel Contributions}

The main highlights and contributions of this thesis are:

- It is shown that the time-implicit GL-DGSEM can be formulated as a Schurcomplement problem and can be efficiently solved using a static-condensation method that retains the advantageous orthogonal basis functions. This strategy allows one to speed up the fluid flow computations (in Chapter 6).

- The derivation of the analytical Jacobian for high-order Discontinuous Galerkin methods is presented. The derivation presented here can be used for any linear or nonlinear equation in conservation form with advective and/or diffusive terms.

- A novel truncation error estimator for the $p$-anisotropic DGSEM is formulated that is computationally cheaper to evaluate and more accurate than previous approaches (in Chapter 8 and [180]).

- A strategy to perform local anisotropic $p$-adaptation for steady flow problems using the novel truncation error estimator is presented. It is shown that this $p$-adaptation strategy can be readily coupled with a multigrid method to yield significant speed-ups (in Chapter 9 and [178]).

- Two approaches to perform local anisotropic $p$-adaptation for unsteady flow problems are developed: a static one and a dynamic one. We demonstrate 
that the use of these approaches leads to shorter computational times (in Chapter 10).

- An efficient DGSEM solver for the 3D compressible Navier-Stokes equations in unstructured grids was developed with the researchers Juan Manzanero and Gonzalo Rubio of the Applied Mathematics Department at ETSIAE-UPM based on David Kopriva's book and its solver [113] (see Appendix C - HORSES3D: A High Order (DG) Spectral Element Solver). HORSES3D offers explicit and implicit time-integration methods. The code can compute the DG Jacobian numerically or analytically for any PDE in conservation form with hyperbolic and/or parabolic terms (useful for implicit time-integration methods, for stability and sensitivity analysis, etc.). Moreover, the code is able to perform static and dynamic anisotropic $p$-adaptation using truncation error estimates for 3D problems.

All the solution techniques that are presented in this thesis are applied to the 3D compressible Navier-Stokes equations, since they represent a complex nonlinear problem that is of interest in aeronautics. However, the methods developed in this thesis can be applied to any system of conservation laws discretized using the DGSEM.

\subsubsection{List of Publications}

Parts of this thesis were published and presented in peer-reviewed scientific journals and conferences. The following publications and dissemination activities were carried out during the development of this thesis:

\subsubsection{Journal Publications}

- Rueda-Ramírez, A. M.; Manzanero, J.; Ferrer, E.; Rubio, G.; Valero, E. (2019). "A $p$-multigrid strategy with anisotropic $p$-adaptation based on truncation errors for high-order discontinuous Galerkin methods". Journal of Computational Physics, 378, 209-233.

- Rueda-Ramírez, A. M.; Rubio, G.; Ferrer, E.; Valero, E. (2019). "Truncation Error Estimation in the $p$-Anisotropic Discontinuous Galerkin Spectral Element Method". Journal of Scientific Computing, 78(1), 433-466.

- Manzanero, J.; Rueda-Ramírez, A. M.; Rubio, G.; Ferrer, E. (2018). "The Bassi Rebay 1 scheme is a special case of the Symmetric Interior Penalty formulation for discontinuous Galerkin discretisations with Gauss-Lobatto points". Journal of Computational Physics, 363, 1-10. 


\subsubsection{Book Chapters}

- Rueda-Ramírez, A. M.; Rubio, G.; Ferrer, E.; Valero, E. "An Anisotropic pAdaptation Multigrid Scheme for the Discontinuous Galerkin Spectral Element Method". Lecture Notes in Computational Science and Engineering (2019). Proceedings of the International Conference on Spectral and HighOrder Methods (ICOSAHOM 2018). London, UK.

- Ferrer, E.; Manzanero, J.; Rueda-Ramírez, A. M.; Rubio, G.; Valero, E. "Implicit large eddy simulations for NACA0012 airfoils using compressible and incompressible discontinuous Galerkin solvers". Proceedings of the International Conference on Spectral and High-Order Methods (ICOSAHOM 2018). London, UK.

\subsubsection{Participation in International Conferences}

- Rueda-Ramírez, A. M.; Rubio, G.; Ferrer, E.; Valero, E. "An Anisotropic pAdaptation Multigrid Scheme for the Discontinuous Galerkin Spectral Element Method". International Conference on Spectral and High-Order Methods (ICOSAHOM 2018). London, UK.

- Rueda-Ramírez, A. M.; Rubio, G.; Ferrer, E.; Valero, E. "An Approach to Unsteady $p$-Adaptation Based on Truncation Error Estimations for High-Order Discontinuous Galerkin Methods". VII European workshop on high order numerical methods for evolutionary PDEs (HONOM 2019). Madrid, Spain.

- Rueda-Ramírez, A. M.; Kopriva, D.A.; Ferrer, E.; Rubio, G.; Valero, E. "A Static Condensation Algorithm for Time-Implicit discretizations of Gauss- Lobatto Discontinuous Galerkin Spectral Element Methods". VII European workshop on high order numerical methods for evolutionary PDEs (HONOM 2019). Madrid, Spain.

- Rueda-Ramírez, A. M.; Rubio, G.; Ferrer, E.; Valero, E. "An Unsteady and Anisotropic $p$-Adaptation Method Based on Truncation Error Estimations for High-Order Discontinuous Galerkin Schemes". North American High-Order Methods Conference (NAHOMCon 2019). San Diego, USA.

- Rueda-Ramírez, A. M.; Kopriva, D.A.; Ferrer, E.; Rubio, G.; Valero, E. "A Time-Implicit Static Condensation Scheme for High-Order Discontinuous Galerkin Spectral Element Methods with Gauss-Lobatto Points". North American High-Order Methods Conference (NAHOMCon 2019). San Diego, USA.

- Ferrer, E.; Manzanero, J.; Rueda-Ramírez, A. M.; Rubio, G.; Valero, E. "Towards robust Large Eddy Simulations using discontinuous Galerkin methods". International Conference on Spectral and High-Order Methods (ICOSAHOM 2018). London, UK. 
- Ferrer, E.; Manzanero, J.; Rueda-Ramírez, A. M.; Rubio, G.; Valero, E.. "Implicit large eddy simulations for airfoils using compressible and incompressible discontinuous Galerkin solvers". VII European workshop on high order numerical methods for evolutionary PDEs (HONOM 2019). Madrid, Spain.

Furthermore, other parts of this thesis are currently under review for publication in international Journals:

- Rueda-Ramírez, A. M.; Ferrer, E.; Kopriva, D.A.; Rubio, G.; Valero, E. "A Static Condensation Algorithm for Time-Implicit Discretizations of Discontinuous Galerkin Spectral Element Methods on Gauss-Lobatto points".

- Rueda-Ramírez, A. M.; Rubio, G.; Ferrer, E.; Valero, E. "An Unsteady and Anisotropic $p$-Adaptation Method Using Truncation Error Estimations for HighOrder Discontinuous Galerkin Schemes".

\subsection{Notation}

This section contains the summary of the several tensor and differentiation operators that are used throughout this thesis.

We distinguish between four kinds of vectors:

1. Spatial vectors of size $d$ (the number of spatial dimensions) are noted with a lowercase letter and an over right-arrow. For example, the velocity vector and the nabla operator are defined, respectively, as

$$
\vec{v}=\left[\begin{array}{c}
v_{1} \\
\vdots \\
v_{d}
\end{array}\right], \quad \vec{\nabla}=\left[\begin{array}{c}
\frac{\partial}{\partial x_{1}} \\
\vdots \\
\frac{\partial}{\partial x_{d}}
\end{array}\right] .
$$

2. State vectors contain $n_{\text {cons }}$ relevant physical variables and are noted with a bold lowercase letter. For example, when solving the 3D compressible NavierStokes equations, we can define the state vector of conservative variables as $\mathbf{q}=[\rho, \rho u, \rho v, \rho w, \rho E]^{T}$, where $n_{\text {cons }}=5$ (see Appendix A).

3. Generic vectors, that are not necessarily spatial or state vectors, are noted with a bold uppercase letter, e.g. Q. In this thesis, generic vectors usually contain the discretized values of a corresponding state vector at different space locations, e.g. $\mathbf{Q}=\left[\mathbf{q}_{1}, \mathbf{q}_{2}, \cdots, \mathbf{q}_{\mathrm{NDOF}}\right]^{T}$.

4. Block vectors contain a state vector in every spatial direction. For instance, a multi-variable flux in three dimensions can be represented as

$$
\overleftrightarrow{\mathbf{f}}=\left[\begin{array}{l}
\mathbf{f}_{1} \\
\mathbf{f}_{2} \\
\mathbf{f}_{3}
\end{array}\right]=\mathbf{f}_{1} \hat{\imath}+\mathbf{f}_{2} \hat{\jmath}+\mathbf{f}_{3} \hat{k},
$$


where every component $\mathbf{f}_{i}$ is a state vector of size $n_{\text {cons }}$. If $n_{\text {cons }}=1$, the flux block vector is a spatial vector (therefore the arrow), and if $n_{\text {cons }}>1$, the flux can also be interpreted as a second order tensor.

In general, we will be dealing with systems of conservation laws in a domain $\Omega \in \mathbb{R}$, which we can now write as

$$
\partial_{t} \mathbf{q}+\vec{\nabla} \cdot \overleftrightarrow{\mathbf{f}}=\mathbf{s}
$$

where $\mathbf{q}$ is the state vector of conserved variables, $\stackrel{\mathbf{f}}{\text { is }}$ the flux, a block vector, and $\mathbf{s}$ is a source term.

The gradient of a state vector is a block vector:

$$
\vec{\nabla} \mathbf{q}=\left[\begin{array}{l}
\partial_{x} \mathbf{q} \\
\partial_{y} \mathbf{q} \\
\partial_{z} \mathbf{q}
\end{array}\right]=\partial_{x} \mathbf{q} \hat{\imath}+\partial_{y} \mathbf{q} \hat{\jmath}+\partial_{z} \mathbf{q} \hat{k}
$$

and the gradient of a spatial vector can be represented as a second order tensor, which can be written in matrix form using the outer product as

$$
\underline{\mathbf{L}}=\vec{\nabla} \vec{v}=(\vec{\nabla} \otimes \vec{v})^{T}=\left(\vec{\nabla} \vec{v}^{T}\right)^{T}=\left[\begin{array}{lll}
\frac{\partial v_{1}}{\partial x} & \frac{\partial v_{1}}{\partial y} & \frac{\partial v_{1}}{\partial z} \\
\frac{\partial v_{2}}{\partial x} & \frac{\partial v_{2}}{\partial y} & \frac{\partial v_{2}}{\partial z} \\
\frac{\partial v_{3}}{\partial x} & \frac{\partial v_{3}}{\partial y} & \frac{\partial v_{3}}{\partial z}
\end{array}\right] .
$$

The underline is used throughout this work for second order tensors and matrices. Third order tensors are noted with a double underline, e.g. the flux derivative with respect to $\mathbf{q}$ can be expressed as

$$
\frac{\partial \overleftrightarrow{\mathbf{f}}}{\partial \mathbf{q}}=\underline{\mathbf{J}}=\left[\begin{array}{l}
\underline{\mathbf{J}}_{1} \\
\underline{\mathbf{J}}_{2} \\
\underline{\mathbf{J}}_{3}
\end{array}\right]=\left[\begin{array}{l}
\frac{\partial \mathbf{f}_{1}}{\partial \mathbf{q}} \\
\frac{\partial \mathbf{f}_{2}}{\partial \mathbf{q}} \\
\frac{\partial \mathbf{f}_{3}}{\partial \mathbf{q}}
\end{array}\right]
$$

Similarly, fourth order tensors are noted with a triple underline. For example, the derivative of the flux with respect to $\vec{\nabla} \mathbf{q}$ can be written as

$$
\frac{\partial \overleftrightarrow{\mathbf{f}}}{\partial(\vec{\nabla} \mathbf{q})}=\underline{\underline{\mathbf{G}}}=\left[\begin{array}{l}
\underline{\mathbf{G}}_{1} \\
\underline{\mathbf{G}}_{2} \\
\underline{\mathbf{G}}_{3}
\end{array}\right]=\left[\begin{array}{c}
\frac{\partial \mathbf{f}_{1}}{\partial(\vec{\nabla} \mathbf{q})} \\
\frac{\partial \mathbf{f}_{2}}{\partial(\vec{\nabla} \mathbf{q})} \\
\frac{\partial \mathbf{f}_{3}}{\partial(\vec{\nabla} \mathbf{q})}
\end{array}\right]=\left[\begin{array}{ccc}
\frac{\partial \mathbf{f}_{1}}{\partial\left(\partial_{x} \mathbf{q}\right)} & \frac{\partial \mathbf{f}_{1}}{\partial\left(\partial_{y} \mathbf{q}\right)} & \frac{\partial \mathbf{f}_{1}}{\partial\left(\partial_{z} \mathbf{q}\right)} \\
\frac{\partial \mathbf{f}_{2}}{\partial\left(\partial_{x} \mathbf{q}\right)} & \frac{\partial \mathbf{f}_{2}}{\partial\left(\partial_{y} \mathbf{q}\right)} & \frac{\partial \mathbf{f}_{2}}{\partial\left(\partial_{z} \mathbf{q}\right)} \\
\frac{\partial \mathbf{f}_{3}}{\partial\left(\partial_{x} \mathbf{q}\right)} & \frac{\partial \mathbf{f}_{3}}{\partial\left(\partial_{y} \mathbf{q}\right)} & \frac{\partial \mathbf{f}_{3}}{\partial\left(\partial_{z} \mathbf{q}\right)}
\end{array}\right]=\left[\begin{array}{lll}
\underline{\mathbf{G}}_{11} & \underline{\mathbf{G}}_{12} & \underline{\mathbf{G}}_{13} \\
\mathbf{G}_{21} & \underline{\mathbf{G}}_{22} & \underline{\mathbf{G}}_{23} \\
\underline{\mathbf{G}}_{31} & \underline{\mathbf{G}}_{32} & \underline{\mathbf{G}}_{33}
\end{array}\right] .
$$

The dot (inner) product of two block vectors is a scalar,

$$
\overleftrightarrow{\mathbf{f}} \cdot \stackrel{\leftrightarrow}{\mathbf{g}}=\sum_{i=1}^{d} \mathbf{f}_{i} \cdot \mathbf{g}_{i},=\sum_{i=1}^{d} \mathbf{f}_{i}^{T} \mathbf{g}_{i}
$$


This operation can also be interpreted as a double dot product when $n_{\text {cons }}>1$, since in that case $\overleftrightarrow{\mathbf{f}}$ is a second order tensor in which one of the ranks has a directional character. Moreover, the dot product of a spatial vector with a block vector is a state vector,

$$
\begin{array}{r}
\vec{v} \cdot \overleftrightarrow{\mathbf{f}}=\sum_{i=1}^{d} v_{i} \mathbf{f}_{i} \\
\vec{\nabla} \cdot \overleftrightarrow{\mathbf{f}}=\sum_{i=1}^{d} \partial_{i} \mathbf{f}_{i}
\end{array}
$$

Finally, the product of a third order tensor by a state vector is a block vector,

$$
\underline{\mathbf{J}} \mathbf{q}=\left[\begin{array}{l}
\underline{\mathbf{J}}_{1} \\
\underline{\mathbf{J}}_{2} \\
\underline{\mathbf{J}}_{3}
\end{array}\right] \mathbf{q}=\left[\begin{array}{l}
\underline{\mathbf{J}}_{1} \mathbf{q} \\
\underline{\mathbf{J}}_{2} \mathbf{q} \\
\underline{\mathbf{J}}_{3} \mathbf{q}
\end{array}\right]
$$

and the product of a fourth order tensor of $d$ columns by a block vector is also a block vector, for example:

$$
\underline{\underline{\underline{\mathbf{G}}}} \underline{\mathbf{g}}=\underline{\underline{\mathbf{G}}} \vec{\nabla} \mathbf{q}=\left[\begin{array}{lll}
\underline{\mathbf{G}}_{11} & \underline{\mathbf{G}}_{12} & \underline{\mathbf{G}}_{13} \\
\underline{\mathbf{G}}_{21} & \underline{\mathbf{G}}_{22} & \underline{\mathbf{G}}_{23} \\
\underline{\mathbf{G}}_{31} & \underline{\mathbf{G}}_{32} & \underline{\mathbf{G}}_{33}
\end{array}\right]\left[\begin{array}{l}
\mathbf{g}_{1} \\
\mathbf{g}_{2} \\
\mathbf{g}_{3}
\end{array}\right]=\left[\begin{array}{l}
\underline{\mathbf{G}}_{11} \mathbf{g}_{1}+\underline{\mathbf{G}}_{12} \mathbf{g}_{2}+\underline{\mathbf{G}}_{13} \mathbf{g}_{3} \\
\underline{\mathbf{G}}_{21} \mathbf{g}_{1}+\underline{\mathbf{G}}_{22} \mathbf{g}_{2}+\underline{\mathbf{G}}_{23} \mathbf{g}_{3} \\
\underline{\mathbf{G}}_{31} \mathbf{g}_{1}+\underline{\mathbf{G}}_{32} \mathbf{g}_{2}+\underline{\mathbf{G}}_{33} \mathbf{g}_{3}
\end{array}\right] .
$$

In several parts of the text, we will deal with volume integrals of a product of two variables ( $L_{2}$ inner products). To simplify the notation, sometimes we will write the $L_{2}$ inner products in compact form as

$$
\begin{aligned}
\langle\overleftrightarrow{\mathbf{f}}, \overleftrightarrow{\mathbf{g}}\rangle_{\Omega} & =\int_{\Omega} \overleftrightarrow{\mathbf{f}} \cdot \stackrel{\leftrightarrow}{\mathbf{g}} \mathrm{d} \Omega \\
\langle\mathbf{f}, \mathbf{g}\rangle_{\Omega} & =\int_{\Omega} \mathbf{f} \cdot \mathbf{g} \mathrm{d} \Omega \\
\langle\mathbf{f}, g\rangle_{\Omega} & =\int_{\Omega} \mathbf{f} g \mathrm{~d} \Omega .
\end{aligned}
$$

If the integral of the $L_{2}$ inner product is computed with a quadrature of order $N$, it is noted as

$$
\langle\overleftrightarrow{\mathbf{f}}, \overleftrightarrow{\mathbf{g}}\rangle_{\Omega}^{N}=\int_{\Omega}^{N} \stackrel{\leftrightarrow}{\mathbf{f}} \cdot \stackrel{\leftrightarrow}{\mathbf{g}} \mathrm{d} \Omega
$$

If $N$ is not enough to evaluate the integral exactly, (1.16) is called an inexact $L_{2}$ inner product.

Different norms are used throughout this work: 
- The Euclidean (or $\ell_{2}$ ) norm is noted as

$$
\begin{gathered}
\|\vec{v}\|=\sqrt{v_{1}^{2}+v_{2}^{2}+v_{3}^{2}}, \\
\|\mathbf{q}\|=\sqrt{q_{1}^{2}+q_{2}^{2}+\cdots+q_{n_{\text {cons }}}^{2}} .
\end{gathered}
$$

- The $L_{2}$ norm on a domain $\Omega$ is defined as

$$
\|\mathbf{q}\|_{2}=\langle\mathbf{q}, \mathbf{q}\rangle_{\Omega}=\int_{\Omega} \mathbf{q} \cdot \mathbf{q} \mathrm{d} \Omega=\int_{\Omega}\|\mathbf{q}\|^{2} \mathrm{~d} \Omega .
$$

- The infinity norm is defined as

$$
\|\mathbf{Q}\|_{\infty}=\max \left\{\left\|\mathbf{q}_{1}\right\|_{\infty}, \cdots,\left\|\mathbf{q}_{\mathrm{NDOF}}\right\|_{\infty}\right\}
$$

where

$$
\|\mathbf{q}\|_{\infty}=\max \left\{\left|q_{1}\right|, \cdots,\left|q_{n_{\text {cons }}}\right|\right\} .
$$

In several parts of this thesis, Einstein notation is used to simplify mathematical expressions. When an index appears twice in a mathematical expression and it is not otherwise defined, it implies summation of the corresponding terms over all the possible values of the index. For example, when the index $i$ can range over the set $\{1,2,3,4\}$, the expression

$$
x=\sum_{i=1}^{4} a_{i} b_{i}=a_{1} b_{1}+a_{2} b_{2}+a_{3} b_{3}+a_{4} b_{4},
$$

is simplified as

$$
x=a_{i} b_{i} .
$$

Finally, in many parts of this thesis we have variables that are discontinuous across surfaces. In such cases, the variable on the inner side is noted with a plus, e.g. $\mathbf{q}^{+}$, the variable on the outer side is noted with a minus, e.g. $\mathbf{q}^{-}$, and the unit outward-pointing normal vectors are defined as $\vec{n}=\vec{n}^{+}=-\vec{n}^{-}$. With all this, we can define the average $\{\bullet\}\}$ and jump $\llbracket \bullet \rrbracket$ operators as

$$
\begin{array}{ll}
\left\{\{\mathbf{q}\}=\frac{1}{2}\left(\mathbf{q}^{+}+\mathbf{q}^{-}\right),\right. & \{\overleftrightarrow{\mathbf{f}}\}=\frac{1}{2}\left(\overleftrightarrow{\mathbf{f}}^{+}+\overleftrightarrow{\mathbf{f}}^{-}\right), \\
\llbracket \mathbf{q} \rrbracket=\mathbf{q}^{+} \vec{n}^{+}+\mathbf{q}^{-} \vec{n}^{-}=\left(\mathbf{q}^{+}-\mathbf{q}^{-}\right) \vec{n}^{+}, & \llbracket \overleftrightarrow{\mathbf{f}} \rrbracket=\overleftrightarrow{\mathbf{f}}^{+} \cdot \vec{n}^{+}+\overleftrightarrow{\mathbf{f}}^{-} \cdot \vec{n}^{-}=\left(\overleftrightarrow{\mathbf{f}}^{+}-\overleftrightarrow{\mathbf{f}}^{-}\right) \cdot \vec{n}^{+} .
\end{array}
$$

Table 1.1 provides a summary of the notation conventions used throughout this thesis. 
Table 1.1: Summary of notation

\begin{tabular}{|c|c|c|}
\hline Example & Characteristics & Type of variable \\
\hline$u$ & Lowercase & Scalars \\
\hline$\vec{v}$ & Lowercase with arrow & Spatial vectors \\
\hline $\mathbf{q}$ & Bold lowercase & State vectors \\
\hline $\mathbf{Q}$ & Bold uppercase & Generic vectors \\
\hline$\overleftrightarrow{\mathrm{f}}$ & $\begin{array}{l}\text { Bold lowercase with double } \\
\text { arrow }\end{array}$ & $\begin{array}{l}\text { Block vectors that contain a state } \\
\text { vector in all spatial dimensions }\end{array}$ \\
\hline F & $\begin{array}{l}\text { Bold uppercase with double } \\
\text { arrow }\end{array}$ & $\begin{array}{l}\text { Block vectors that contain a } \\
\text { generic vector in each spatial di- } \\
\text { mension }\end{array}$ \\
\hline$\underline{\mathbf{M}}, \underline{\tau}$ & $\begin{array}{l}\text { Underlined and, if it is a ro- } \\
\text { man letter, bold uppercase }\end{array}$ & $\begin{array}{l}\text { Matrices and second order ten- } \\
\text { sors }\end{array}$ \\
\hline$\underline{\text { B }}$ & Bold with double underline & Third order tensors \\
\hline$\underline{\mathbf{G}}$ & Bold with triple underline & Fourth order tensors \\
\hline
\end{tabular}





\section{Part I \\ Background: Discretization Methods}

Summary This part provides a general overview of the discretization methods that are used throughout the thesis. Chapter 2 details the spatial discretization strategy: the formulation of the arbitrary high-order Discontinuous Galerkin Spectral Element Method for three-dimensional nonlinear problems is presented and its extension to complex geometries using a high-order curvilinear mapping is explained. Chapter 3 addresses the temporal discretization. A brief survey of timemarching schemes is presented, and the accuracy, stability properties and computational cost of the different methods is discussed. 

${ }_{\text {Chapter }} 2$

\section{Spatial Discretization}

\section{Contents}

2.1 The Discontinuous Galerkin Discretization . . . . . . . . . . 19

2.1.1 Discretization of Viscous Terms . . . . . . . . . . 22

2.1 .2 Advective Numerical Flux . . . . . . . . . . . . . 23

2.1 .3 Diffusive Numerical Flux . . . . . . . . . . . . . . 24

2.2 The Discontinuous Galerkin Spectral Element Method (DGSEM) 25

2.2.1 Curvilinear Mapping . . . . . . . . . . . . . 27

\subsection{The Discontinuous Galerkin Discretization}

We consider the approximation of systems of conservation laws,

$$
\partial_{t} \mathbf{q}+\vec{\nabla} \cdot \stackrel{\leftrightarrow}{\mathbf{f}}=\mathbf{s}, \text { in } \Omega,
$$

subject to appropriate boundary conditions, where $\mathbf{q}$ is the state vector of $n_{\text {cons }}$ conserved variables, $\overleftrightarrow{\mathbf{f}}$ is the flux block vector which depends on $\mathbf{q}$ (and possibly on $\mathrm{n}^{\text {th }}$ order spatial derivatives of $\mathbf{q}$ ), and $\mathbf{s}$ is a source term. As detailed in Appendix A, the compressible Navier-Stokes equations can be represented using (2.1).

Multiplying (2.1) by an arbitrary and smooth test function, $\mathbf{v}$, and integrating by parts over the domain, $\Omega$, yields the weak formulation,

$$
\int_{\Omega} \partial_{t} \mathbf{q} \cdot \mathbf{v d} \Omega-\int_{\Omega} \overleftrightarrow{\mathbf{f}} \cdot \vec{\nabla} \mathbf{v d} \Omega+\int_{\partial \Omega}(\overleftrightarrow{\mathbf{f}} \cdot \vec{n}) \cdot \mathbf{v d} S=\int_{\Omega} \mathbf{s} \cdot \mathbf{v d} \Omega,
$$

where $\vec{n}$ is the normal unit vector on the boundary $\partial \Omega$. Let the domain $\Omega$ be approximated by a tessellation $\mathscr{T}=\{e\}$, i.e. a combination of $K$ finite elements $e$ of domain $\Omega^{e}$ and boundary $\partial \Omega^{e}$. 


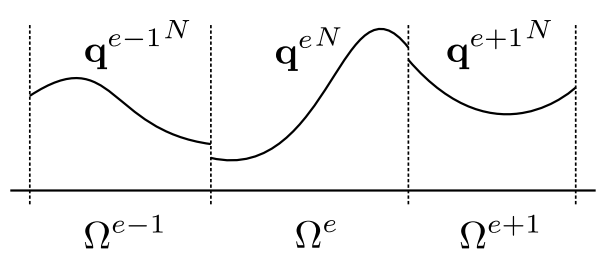

Figure 2.1: Example of a 1D domain subdivision into finite elements with piecewise solutions.

Moreover, let $\mathbf{q}, \mathbf{s}, \stackrel{\leftrightarrow}{\mathbf{f}}$ and $\mathbf{v}$ be approximated by piece-wise polynomial functions $\mathbf{q}^{N}, \mathbf{s}^{N}, \overleftrightarrow{\mathbf{f}}^{N}$ and $\mathbf{v}^{N}$ (which are continuous in each element) defined in the space of $L^{2}$ functions

$$
\mathscr{V}^{N}=\left\{\mathbf{v}^{N} \in L^{2}(\Omega):\left.\mathbf{v}^{N}\right|_{\Omega^{e}} \in \mathscr{P}^{N}\left(\Omega^{e}\right) \forall \Omega^{e} \in \mathscr{T}\right\},
$$

where $\mathscr{P}^{N}\left(\Omega^{e}\right)$ is the space of polynomials of degree at most $N$ defined in the domain of the element $e$ (see Figure 2.1). We remark that the polynomial order $N$ may be different in each element and each local coordinate direction.

Since the functions in $\mathscr{V}^{N}$ may be discontinuous at element interfaces, the quantity $\overleftrightarrow{\mathbf{f}}^{N} \cdot \vec{n}$ is not uniquely defined at the element traces. Therefore, it is replaced by a numerical flux function, $\overleftrightarrow{\mathbf{f}}^{N} \cdot \vec{n} \leftarrow \hat{\mathbf{f}}$. Equation (2.2) can then be rewritten for each element as

$$
\int_{\Omega^{e}} \partial_{t} \mathbf{q}^{e N} \cdot \mathbf{v}^{e N} \mathrm{~d} \Omega^{e}-\int_{\Omega^{e}} \stackrel{\leftrightarrow}{\mathbf{f}} e^{N} \cdot \vec{\nabla} \mathbf{v}^{e N} \mathrm{~d} \Omega^{e}+\int_{\partial \Omega^{e}} \hat{\mathbf{f}} \cdot \mathbf{v}^{e N} \mathrm{~d} S^{e}=\int_{\Omega^{e}} \mathbf{s}^{e N} \cdot \mathbf{v}^{e N} \mathrm{~d} \Omega^{e},
$$

where the superindex $e$ refers to the functions as evaluated inside the element $e$, i.e. $\mathbf{q}^{e N}=\left.\mathbf{q}^{N}\right|_{\Omega^{e}}$. Note that the test function is now only required to be smooth inside the elements and can exhibit discontinuities at element interfaces. The numerical flux function, $\hat{\mathbf{f}}$, allows one to uniquely define the flux at the element interfaces and to weakly prescribe the boundary data as a function of the state on both sides of the boundary/interface and the normal vector. The choice of the numerical flux function is detailed in Sections 2.1.2 and 2.1.3.

Since $\mathbf{q}^{N}, \mathbf{s}^{N}, \mathbf{v}^{N}$ and $\overleftrightarrow{\mathbf{f}}^{N}$ belong to the polynomial space $\mathscr{V}^{N}$, it is possible to express them inside every element as a linear combination of basis functions $\phi_{j} \in$ $\mathscr{P}^{N}\left(\Omega^{e}\right)$ :

$$
\begin{aligned}
& \left.\mathbf{q}\right|_{\Omega^{e}} \approx \mathbf{q}^{e N}=\sum_{j=1}^{\mathrm{NDOF}^{e}} \mathbf{q}_{j}^{e N} \phi_{j}^{e}(\mathbf{x}),\left.\quad \mathbf{s}\right|_{\Omega^{e}} \approx \mathbf{s}^{e N}=\sum_{j=1}^{\mathrm{NDOF}^{e}} \mathbf{s}_{j}^{e N} \phi_{j}^{e}(\mathbf{x}), \\
& \left.\mathbf{v}\right|_{\Omega^{e}} \approx \mathbf{v}^{e N}=\sum_{j=1}^{\mathrm{NDOF}^{e}} \mathbf{v}_{j}^{e N} \phi_{j}^{e}(\mathbf{x}),\left.\quad \overleftrightarrow{\mathbf{f}}\right|_{\Omega^{e}} \approx \stackrel{\leftrightarrow}{\mathbf{f}}^{e^{N}}=\sum_{j=1}^{\mathrm{NDOF}^{e}} \stackrel{\overleftrightarrow{\mathbf{f}}_{j}^{e^{N}}}{\phi_{j}^{e}}(\mathbf{x})
\end{aligned}
$$

where the (spatial) number of degrees of freedom in hexahedral elements depends 
on the polynomial order of the approximation,

$$
\mathrm{NDOF}^{e}=\prod_{i=1}^{d}\left(N_{i}^{e}+1\right)
$$

Since the test function $\mathbf{v}^{N}$ is an arbitrary polynomial, (2.4) must hold for every basis function $\phi_{j}$ and can be rewritten as

$$
\int_{\Omega^{e}} \partial_{t} \mathbf{q}^{e N} \phi_{j}^{e} \mathrm{~d} \Omega^{e}-\int_{\Omega^{e}} \stackrel{\leftrightarrow}{\mathbf{f}} e^{N} \cdot \vec{\nabla} \phi_{j}^{e} \mathrm{~d} \Omega^{e}+\int_{\partial \Omega^{e}} \hat{\mathbf{f}} \phi_{j}^{e} \mathrm{~d} S^{e}=\int_{\Omega^{e}} \mathbf{s}^{e N} \phi_{j}^{e} \mathrm{~d} \Omega^{e},
$$

or in inner product notation as

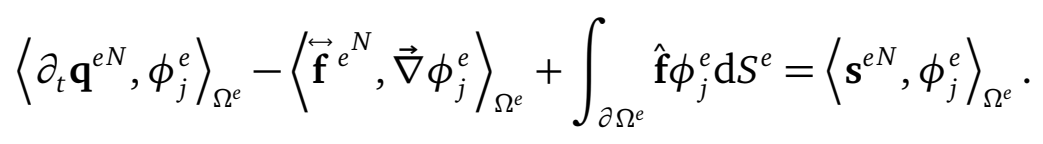

Some authors integrate by parts once again to obtain the equivalent system,

$$
\left\langle\partial_{t} \mathbf{q}^{e N}, \phi_{j}^{e}\right\rangle_{\Omega^{e}}+\left\langle\vec{\nabla} \cdot \stackrel{\leftrightarrow}{\mathbf{f}} e^{N}, \phi_{j}^{e}\right\rangle_{\Omega^{e}}+\int_{\partial \Omega^{e}}\left(\hat{\mathbf{f}}-\overleftrightarrow{\mathbf{f}}^{e^{N}} \cdot \vec{n}\right) \phi_{j}^{e} \mathrm{~d} S^{e}=\left\langle\mathbf{s}^{e N}, \phi_{j}^{e}\right\rangle_{\Omega^{e}} .
$$

Equations (2.7) and (2.8) are usually called the weak DG formulation, and (2.9) is referred to as the weak-strong DG formulation since the volume integral has the original strong form (the differentiation operator is applied on the flux and not on the shape function).

In general, the DG discretized system can be expressed inside each element as

$$
\underline{\mathbf{M}}^{e} \frac{\partial \mathbf{Q}^{e}}{\partial t}+\mathbf{H}^{e}(\mathbf{Q})=\underline{\mathbf{M}}^{e} \mathbf{S}^{e}
$$

where $\mathbf{Q}^{e}=\left[\mathbf{q}_{1}^{e N}, \mathbf{q}_{2}^{e N}, \cdots, \mathbf{q}_{\mathrm{NDOF}^{e}}^{e N}\right]^{T}$ is the local solution that contains the coefficients of the linear combination for the element $e, \mathbf{Q}=\left[\mathbf{Q}^{1}, \mathbf{Q}^{2}, \cdots, \mathbf{Q}^{K}\right]^{T}$ is the global solution that contains the information of all elements, $\underline{\mathbf{M}}^{e}$ is known as the elemental mass matrix, and $\mathbf{H}^{e}=\left[\mathbf{h}_{1}^{e N}, \mathbf{h}_{2}^{e N}, \cdots, \mathbf{h}^{e N}{ }_{\mathrm{NDOF}^{e}}\right]^{T}$ is a nonlinear spatial discrete operator on the element level. Using the weak DG formulation, the operators are

$$
\begin{aligned}
\underline{\mathbf{M}}_{i, j}^{e} & =\underline{\mathbf{I}}_{n_{\text {cons }}} \int_{\Omega^{e}} \phi_{i}^{e} \phi_{j}^{e} \mathrm{~d} \Omega^{e}, \\
\mathbf{h}_{j}^{e}(\mathbf{Q}) & =\sum_{i}\left[-\int_{\Omega^{e}} \phi_{i}^{e \stackrel{\leftrightarrow}{\mathbf{f}^{e^{e}}}} e^{N} \cdot \vec{\nabla} \phi_{j}^{e} \mathrm{~d} \Omega^{e}\right]+\int_{\partial \Omega^{e}} \hat{\mathbf{f}} \phi_{j}^{e} \mathrm{~d} S^{e} .
\end{aligned}
$$

Note that the operator $\mathbf{h}^{e}$ is applied on the global solution since it is the responsible, through the numerical flux, of connecting the elements of the mesh (weakly). Assembling the contributions of all elements into the global system we obtain

$$
\underline{\mathbf{M}} \frac{\partial \mathbf{Q}}{\partial t}+\mathbf{H}(\mathbf{Q})=\underline{\mathbf{M S}} \text {. }
$$




\subsubsection{Discretization of Viscous Terms}

As was stated before, the flux block vector may depend on $\mathrm{n}^{\text {th }}$ order spatial derivatives of q. In this thesis, we restrict the analysis to general nonlinear advectiondiffusion conservation laws (although most of the work can be generalized to any PDE set in conservative form), where the flux has the form,

$$
\overleftrightarrow{\mathbf{f}}(\mathbf{q}, \vec{\nabla} \mathbf{q})=\overleftrightarrow{\mathbf{f}}^{a}(\mathbf{q})-\overleftrightarrow{\mathbf{f}}^{v}(\mathbf{q}, \vec{\nabla} \mathbf{q})
$$

Here, $\overrightarrow{\mathbf{f}}^{a}$ is called the advective (or convective) flux, and $\overleftrightarrow{\mathbf{f}}^{v}$ is called the diffusive (or viscous) flux. The viscous flux requires the computation of an extra spatial derivative, which must be discretized using a DG approach for the approximation to be consistent.

There are multiple ways to discretize the extra spatial derivative, all of which imply splitting the original system of conservation laws into two equations. These two equations can later be assembled as one using static condensation, as shown by Arnold [6]. In this thesis, we use the conservative gradients approach. We will show that this approach provides flexibility to represent a large number of physical phenomena, and that it is easy to obtain the analytical Jacobian for implicit time-integration methods. A comparison of this approach with two other common possibilities (the primitive gradients approach and the whole flux approach) and a discussion of the advantages and disadvantages of each strategy can be found in Appendix B.

The conservative gradients approach defines a block vector, $\overleftrightarrow{\mathbf{g}}$, as the gradient of the conserved variables,

$$
\overleftrightarrow{\mathbf{g}}=\vec{\nabla} \mathbf{q}
$$

so that (2.1) can be rewritten as a system of two equations,

$$
\left\{\begin{array}{rlr}
\partial_{t} \mathbf{q}+\vec{\nabla} \cdot\left(\overrightarrow{\mathbf{f}}^{a}(\mathbf{q})-\overleftrightarrow{\mathbf{f}}^{v}(\mathbf{q}, \overleftrightarrow{\mathbf{g}})\right) & =\mathbf{s}, & \text { in } \Omega, \\
\vec{\nabla} \mathbf{q} & =\overleftrightarrow{\mathbf{g}}, & \text { in } \Omega .
\end{array}\right.
$$

The DG formulation of the first equation of system (2.16) is identical to the one obtained in the previous section. Following a similar procedure, we can obtain the DG formulation for the second equation as

$$
\begin{aligned}
\left\langle\overleftrightarrow{\mathbf{g}}^{N}, \phi_{j}\right\rangle_{\Omega^{e}} & =-\left\langle\mathbf{q}^{N}, \vec{\nabla} \phi_{j}^{e}\right\rangle_{\Omega^{e}}+\int_{\partial \Omega^{e}} \phi_{j}^{e} \hat{\mathbf{q}} \vec{n} \mathrm{~d} S \\
& =\left\langle\vec{\nabla} \mathbf{q}^{N}, \phi_{j}^{e}\right\rangle_{\Omega^{e}}+\int_{\partial \Omega^{e}} \phi_{j}^{e}\left(\hat{\mathbf{q}}-\mathbf{q}^{e N}\right) \vec{n} \mathrm{~d} S,
\end{aligned}
$$

where $\hat{\mathbf{q}}$ is a second numerical flux (actually the numerical trace of the solution) that corresponds to the interface value assumed by the state vector $\mathbf{q}$. Since $\mathbf{q}$ may be discontinuous at element interfaces, there are multiple strategies to compute 
$\hat{\mathbf{q}}$ (some insight is given in Section 2.1.3). Note that, here, the definition of the numerical flux does not include the action of the normal vector. Equation (2.17) corresponds to the weak discretization and (2.18) corresponds to the equivalent weak-strong discretization.

\subsubsection{Advective Numerical Flux}

The numerical flux is in the heart of the DG method and is what really separates it from the classical continuous finite element method and brings it closer to finite volume methods. In essence, the advective numerical flux function provides a solution, or at least an approximate solution, to the Riemann problem that arises because of the discontinuity at elements' interfaces. Therefore, it depends on the solution on the inner side of the element boundary, $\mathbf{q}^{+}$, the solution on the outer side of it, $\mathbf{q}^{-}$, and the outward-pointing normal vector, $\vec{n}$.

By a careful design of the numerical flux function, it is possible to mimic the underlying physics of wave propagation problems and ensure stability. An appropriate numerical flux is required to be [95] (i) consistent, i.e. $\hat{\mathbf{f}}^{a}\left(\mathbf{q}^{+}, \mathbf{q}^{+}, \vec{n}\right)=\overleftrightarrow{\mathbf{f}^{a}}\left(\mathbf{q}^{+}\right) \cdot \vec{n}$, and (ii) conservative, i.e. $\hat{\mathbf{f}}^{a}\left(\mathbf{q}^{+}, \mathbf{q}^{-}, \vec{n}^{+}\right)=\hat{\mathbf{f}}^{a}\left(\mathbf{q}^{-}, \mathbf{q}+, \vec{n}^{-}\right)$. A desirable property is that the discretization reduces to a monotone scheme in the low-order (finite volume) limit, which is ensured if $\hat{\mathbf{f}}^{a}\left(\mathbf{q}^{+}, \mathbf{q}^{-}, \vec{n}\right)$ is nondecreasing in the first argument and nonincreasing in the second, for scalar arguments [95].

Many choices for the numerical flux functions can be found in the finite volume literature [124, 170, 171, 193]. In this section, we only briefly introduce two popular numerical fluxes that are used throughout this thesis.

- The Lax-Friedrichs flux is defined as

$$
\begin{aligned}
\hat{\mathbf{f}}_{L F}^{a}\left(\mathbf{q}^{+}, \mathbf{q}^{-}, \vec{n}\right) & =\frac{1}{2}\left(\overleftrightarrow{\mathbf{f}^{a}}\left(\mathbf{q}^{+}\right) \cdot \vec{n}+\overleftrightarrow{\mathbf{f}}^{a}\left(\mathbf{q}^{-}\right) \cdot \vec{n}+\lambda\left(\mathbf{q}^{+}-\mathbf{q}^{-}\right)\right), \\
& =\left\{\left\{\overleftrightarrow{\mathbf{f}}^{a^{N}}\right\} \cdot \vec{n}+\frac{\lambda}{2} \llbracket \mathbf{q}^{N} \rrbracket\right.
\end{aligned}
$$

where $\lambda=\max \left(\tilde{\lambda}\left(\mathbf{q}^{+}\right), \tilde{\lambda}\left(\mathbf{q}^{-}\right)\right)$is the largest eigenvalue of the linearized advective flux over $\mathbf{q}^{+}$and $\mathbf{q}^{-}$. For example, for the compressible Euler/NavierStokes equations we have,

$$
\tilde{\lambda}(\mathbf{q})=\left|v_{n}(\mathbf{q})\right|+c(\mathbf{q})
$$

with $v_{n}=\vec{v} \cdot \vec{n}$, the normal velocity to the element boundary, and $c=\sqrt{\gamma p / \rho}$, the local speed of sound.

The Lax-Friedrichs flux is one of the simplest approximate Riemann solvers available. However, it can be too dissipative in certain flow conditions [193]. 
- The original Roe flux [170] is defined as

$$
\hat{\mathbf{f}}_{\text {Roe }}^{a}\left(\mathbf{q}^{+}, \mathbf{q}^{-}, \vec{n}\right)=\left\{\stackrel{\mathfrak{f}^{a}}{a^{N}}\right\} \cdot \vec{n}-\frac{1}{2} \sum_{i=1}^{n_{\text {cons }}} \tilde{\alpha}_{i}\left|\tilde{\lambda}_{i}\right| \tilde{\mathbf{k}}^{(i)}
$$

where the $\tilde{\alpha}_{i}$ are averaged wave strengths, the $\tilde{\lambda}_{i}$ are average eigenvalues, and the $\tilde{\mathbf{k}}^{(i)}$ are averaged right eigenvectors in the normal direction. There are different strategies for obtaining these parameters, being the most known the original one by Roe [170], and the one by Roe and Pike [171].

In transonic or sonic regimes, the traditional Roe solver encounters problems and describes spurious shocks. Therefore, entropy fixes have been introduced to the original model by Harten and Hyman [90] Roe and Pike [171], among others.

The notation with plus and minus signs for the inner and outer values of the solution, respectively, is broadly used in the DG community (in some cases reversed) and therefore it is retained. However, note that a choice of arguments that is consistent with the derivations of last section and that is valid for an inner element interface holds,

$$
\hat{\mathbf{f}}^{a}\left(\mathbf{q}^{+}, \mathbf{q}^{-} \cdot \vec{n}\right)=\hat{\mathbf{f}}^{a}\left(\mathbf{q}^{e N}, \mathbf{q}^{N} \cdot \vec{n}\right),
$$

where $\mathbf{q}^{\underline{N}}$ is the discrete solution on a neighbor element $(\neq e)$ that is connected through $\partial \Omega$. In the rest of this thesis, both notation conventions will be used interchangeably as seen fit for readability.

In addition, note that the superindex $N$ is not used for $\hat{\mathbf{f}}$, since the presence of a numerical flux implies that $\overleftrightarrow{\mathbf{f}} \cdot \vec{n}$ is undefined, i.e. that we are dealing with the discrete representation.

\subsubsection{Diffusive Numerical Flux}

The literature on diffusive numerical fluxes for DG methods is also extensive [6, 14, 15, 56]. Arnold et al. [6] provide a unified analysis of a variety of diffusive numerical fluxes available in the literature and classifies them in two categories: consistent and stable methods. Here, we describe two viscous numerical fluxes that are used throughout the document.

- The scheme by Bassi and Rebay [14] (BR1) is probably the simplest diffusive Riemann solver as it defines the intermediate states as simple averages of the states on both sides of the interface:

$$
\begin{aligned}
\hat{\mathbf{q}} & =\left\{\left\{\mathbf{q}^{N}\right\},\right. \\
\hat{\mathbf{f}}^{v} & =\left\{\left\{\overleftrightarrow{\mathbf{f}}^{v N}\left(\mathbf{q}^{N}, \overleftrightarrow{\mathbf{g}}^{N}\right)\right\} \cdot \vec{n} .\right.
\end{aligned}
$$


In [6], Arnold et al. classify the BR1 scheme as consistent, but not stable for purely elliptic problems. However, the scheme has been proved to be stable for the compressible Navier-Stokes equations [79].

- The symmetric interior penalty (IP) method [56] defines the viscous numerical fluxes as

$$
\begin{aligned}
\hat{\mathbf{q}} & =\left\{\left\{\mathbf{q}^{N}\right\},\right. \\
\hat{\mathbf{f}}^{v} & =\left(\left\{\stackrel{\leftrightarrow}{\mathbf{f}^{\nu N}}\left(\mathbf{q}^{N}, \vec{\nabla} \mathbf{q}^{N}\right)\right\}-\delta \llbracket \mathbf{q}^{N} \rrbracket\right) \cdot \vec{n},
\end{aligned}
$$

where $\delta$ is a penalty parameter that depends on the mesh and the viscosity. The most used expression for the penalty parameter is the one proposed by Shahbazi et al. [184], but others can be found in the literature, such as in $[65,138,168]$.

From the form of $\hat{\mathbf{f}}^{v}$, we can classify the diffusive numerical fluxes as compact, when they depend on $\vec{\nabla} \mathbf{q}^{N}$, or non-compact, when they depend on $\overleftrightarrow{\mathbf{g}}^{N}=(\vec{\nabla} \mathbf{q})^{N}$. The main difference is that in the non-compact schemes, the viscous numerical flux depends on the DG gradients of neighbor elements, which in turn depend on the solution of their neighbors through (2.17) or (2.18). This results in a widened stencil, as will be illustrated in Section 5.4.

It has been proved that the BR1 scheme is a special case of the symmetric IP method for Discontinuous Galerkin Spectral Element Methods (see Section 2.2) on Gauss-Lobatto points, both schemes being compact in Cartesian meshes. For the proof, see [138].

\subsection{The Discontinuous Galerkin Spectral Element Method (DGSEM)}

The Discontinuous Galerkin Spectral Element Method [23, 113] is a nodal (collocation) version of the DG discretization in which the tessellation is performed with non-overlapping quadrilateral (2D) or hexahedral (3D) elements of order $N=\left(N_{1}, N_{2}, N_{3}\right)$ (independent in every local coordinate direction), and the integrals are evaluated numerically by means of a Gaussian quadrature rule of order $N$. In 1D, the quadrature rule is

$$
\int_{-1}^{1} f \mathrm{~d} \xi \approx \int_{-1}^{1^{N}} f \mathrm{~d} \xi=\sum_{i=0}^{N} w_{i} f\left(\xi_{i}\right)
$$

where $\xi_{i}$ and $w_{i}$ are the positions and weights of the quadrature nodes. For volume integrals, (2.28) is easily extended to 3D using tensor products,

$$
\int_{-1}^{1 N_{3}} \int_{-1}^{1 N_{2}} \int_{-1}^{1^{N_{1}}} f \mathrm{~d} \xi \mathrm{d} \eta \mathrm{d} \zeta=\sum_{i=0}^{N_{1}} \sum_{j=0}^{N_{2}} \sum_{k=0}^{N_{3}} w_{i} w_{j} w_{k} f\left(\xi_{i}, \eta_{j}, \zeta_{k}\right) .
$$


Furthermore, in the DGSEM the polynomial basis functions, $\phi_{j}$, are tensorproduct reconstructions of Lagrange interpolating polynomials on the quadrature points in each local coordinate direction:

$$
\mathbf{q}^{e N}(\vec{\xi})=\sum_{n=1}^{\mathrm{NDOF}^{e}} \mathbf{q}_{n}^{e N} \phi_{n}^{e}(\vec{\xi})=\sum_{i=0}^{N_{1}} \sum_{j=0}^{N_{2}} \sum_{k=0}^{N_{3}} \mathbf{q}_{i, j, k}^{e N} \ell_{i}^{\xi}(\xi) \ell_{j}^{\eta}(\eta) \ell_{k}^{\zeta}(\zeta),
$$

and the Lagrange polynomials are

$$
\ell_{i}^{\xi}(\xi)=\prod_{\substack{m=0 \\ m \neq i}}^{N_{1}} \frac{\xi-\xi_{m}}{\xi_{i}-\xi_{m}}
$$

Therefore, $\mathbf{q}_{n}^{e N}=\mathbf{q}_{i, j, k}^{e N}$ are simply the nodal values of the solution, on the Gauss or Gauss-Lobatto nodes. This facilitates the computation of solution-dependent parameters, such as the viscosity in the compressible Navier-Stokes equations.

The standard choices for the quadrature rule are the Legendre-Gauss and the Legendre-Gauss-Lobatto nodes [113] (usually called only Gauss or Gauss-Lobatto points, respectively). Figure 2.2 shows the Lagrange interpolating polynomials on both quadrature rules for a $1 \mathrm{D}$ discretization with $N=4$. Note that the Lagrange interpolating polynomials are discretely orthogonal,

$$
\ell_{i}^{\xi}\left(\xi_{j}\right)=\delta_{i j}
$$

Therefore, the mass matrix, $\underline{\mathbf{M}}$, is a diagonal matrix containing only the quadrature weights.

The Gauss quadrature rule provides exact integration for polynomials of up to order $2 N+1$, whereas the Gauss-Lobatto quadrature is only exact for polynomials up to order $2 N-1$ [113].

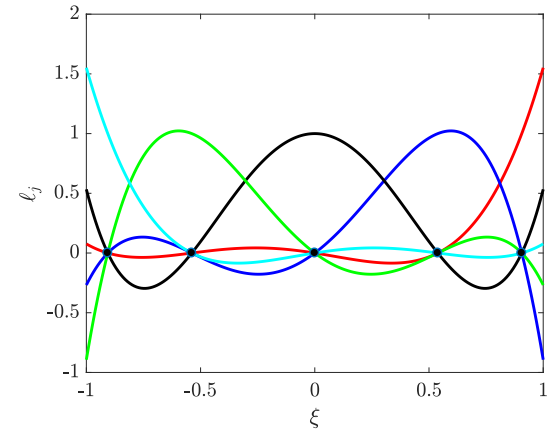

(a) Legendre-Gauss.

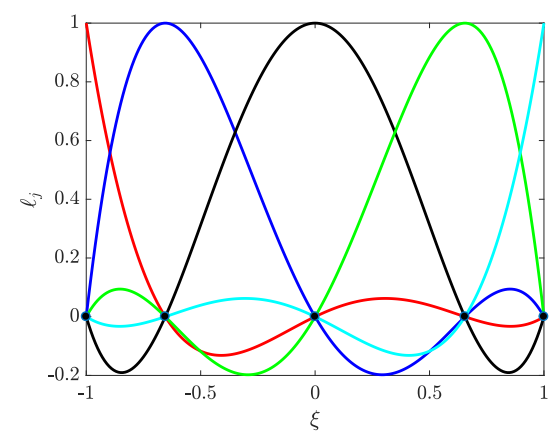

(b) Legendre-Gauss-Lobatto.

Figure 2.2: Lagrange interpolating polynomials on Legendre-Gauss and LegendreGauss-Lobatto points. 


\subsubsection{Curvilinear Mapping}

Since the simulation domain rarely corresponds to the domain where the quadrature rules are defined, i.e. $[-1,1]^{d}$, it is most convenient to perform the numerical integration in a reference element and transform the results to the physical space by means of a high-order mapping of order $M=\left(M_{1}, M_{2}, M_{3}\right)$ :

$$
\vec{x}^{e}=\vec{x}^{e}(\vec{\xi}) \in \mathscr{P}^{M}, \quad \vec{\xi}=(\xi, \eta, \zeta) \in[-1,1]^{3} .
$$

This also allows one to describe curved boundaries accurately.

The differential operators can be expressed in the reference element in terms of the covariant $\left(\vec{a}_{i}\right)$ and contravariant $\left(\vec{a}^{i}\right)$ metric tensors [113]:

$$
\vec{a}_{i}=\frac{\partial \vec{x}^{e}}{\partial \xi_{i}}, \quad \vec{a}^{i}=\vec{\nabla} \vec{\xi}_{i}, \quad i=1,2,3
$$

Using these mappings, the gradient and divergence operators become [26, 113]

$$
\vec{\nabla} \mathbf{q}=\frac{1}{J} \underline{\mathscr{M}}^{T} \vec{\nabla}_{\xi} \mathbf{q}, \quad \vec{\nabla} \cdot \stackrel{\leftrightarrow}{\mathbf{f}}=\frac{1}{J} \vec{\nabla}_{\xi} \cdot \overleftrightarrow{\tilde{\mathbf{f}}}=\frac{1}{J} \vec{\nabla}_{\xi} \cdot\left(\underline{\mathscr{M}}^{T} \overleftrightarrow{\mathbf{f}}\right)
$$

where $\stackrel{\overleftrightarrow{\mathrm{f}}}{\text { is }}$ called the contravariant flux, the determinant of the Jacobian of the transformation can be expressed in terms of the covariant metric tensor,

$$
J=\vec{a}_{i} \cdot\left(\vec{a}_{j} \times \vec{a}_{k}\right), \quad(i, j, k) \text { cyclic, }
$$

and the matrix $\underline{\mathscr{M}}$ is defined as

$$
\underline{\mathscr{M}}=\left[\begin{array}{lll}
J a_{1}^{1} \underline{\mathbf{I}}_{n_{\text {cons }}} & J a_{1}^{2} \underline{\mathbf{I}}_{n_{\text {cons }}} & J a_{1}^{3} \mathbf{I}_{n_{\text {cons }}} \\
J a_{2}^{1} \underline{\mathbf{I}}_{n_{\text {cons }}} & J a_{2}^{2} \underline{\mathbf{I}}_{\text {cons }} & J a_{2}^{3} \mathbf{I} n_{\text {cons }} \\
J a_{3}^{1} \underline{\mathbf{I}}_{\text {cons }} & J a_{3}^{2} \underline{\mathbf{I}}_{\text {cons }} & J a_{3}^{3} \underline{\mathbf{I}}_{\text {cons }}
\end{array}\right],
$$

an expanded version of the Jacobian matrix $\partial \vec{\xi} / \partial \vec{x}$. With this consideration, the DGSEM system for the degree of freedom $j$ of element $e$ becomes

$$
\begin{array}{r}
J_{j} w_{j} \partial_{t} \mathbf{q}_{j}^{e N}-\int_{\Omega_{\xi}}^{N} \stackrel{\overleftrightarrow{\mathbf{f}}^{e}}{e^{N}} \cdot \vec{\nabla}_{\xi} \phi_{j}^{e} \mathrm{~d} \Omega+\int_{\partial \Omega_{\xi}}^{N} J \hat{\mathbf{f}} \phi_{j}^{e} \mathrm{~d} S=J_{j} w_{j} \mathbf{s}_{j}^{e N}, \\
-\int_{\Omega_{\xi}}^{N} J \mathbf{q}^{N} \underline{\mathscr{M}}^{T} \vec{\nabla} \phi_{j}^{e} \mathrm{~d} \Omega^{\xi}+\int_{\partial \Omega_{\xi}}^{N} J \phi_{j}^{e} \hat{\mathbf{q}} \overrightarrow{\mathrm{d}} \mathrm{d} S=J_{j} w_{j} \overleftrightarrow{\mathbf{g}}_{j}^{N} .
\end{array}
$$

Note that, because of the curvilinear mapping, the mass matrix does not only contain the quadrature weights, but also the determinant of the Jacobian of the transformation.

After defining the high-order mapping equations, the covariant vectors can be readily obtained from the mapping (2.33). For $2 \mathrm{D}$ problems, the contravariant 
vectors can be obtained with the well-known "cross product form" [112]. However, for fully 3D problems, the contravariant vectors must be obtained using either the "conservative curl form" or the "invariant curl form" [112]. Since in this work we deal with 3D curved meshes, the "invariant curl form" is selected,

$$
J a_{n}^{i}=-\frac{1}{2} \hat{i}_{i} \cdot \vec{\nabla}_{\xi} \times\left[\mathbf{I}^{N}\left(x_{l} \vec{\nabla}_{\xi} x_{m}-x_{m} \vec{\nabla}_{\xi} x_{l}\right)\right] \quad i=1,2,3, n=1,2,3, \quad(n, m, l) \text { cyclic, }
$$

where $\mathbf{I}^{N}$ is the interpolating operator that converts an arbitrary continuous function into a polynomial expansion (as in (2.5)).

As a final remark, in the DGSEM the order of the mapping, $M$ in (2.33), must be $M_{i} \leq N_{i}$ for 2D, 2D-extruded and 3D $p$-conforming discretizations (subparametric or at most isoparametric mapping) to retain free-stream-preservation [112], whereas it is limited to $M_{i} \leq N_{i} / 2$ for general 3D nonconforming discretizations [114]. Details on how to implement the mappings and, in general, all the explicit DGSEM operators can be obtained in [113]. 


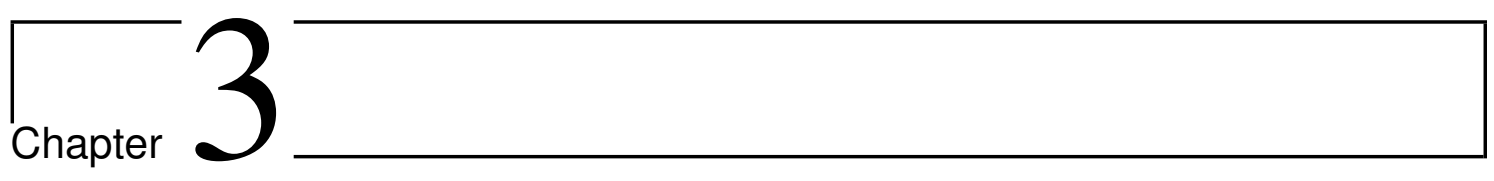

\section{Temporal Discretization}

\section{Contents}

3.1 General Properties . . . . . . . . . . . . . . . . 30

3.2 Explicit Time-Integration $\ldots \ldots \ldots \ldots \ldots \ldots \ldots \ldots$

3.2.1 Explicit Runge-Kutta Methods . . . . . . . . . . . . 32

3.2 .2 Stability ................... 33

3.3 Implicit Time-Integration $\ldots \ldots \ldots \ldots \ldots \ldots \ldots$

3.3.1 Backward Differentiation Formulas (BDFs) $\ldots \ldots \ldots \ldots$

After the spatial discretization is completed (Chapter 2), we obtain a system of equations that describes an initial value problem (IVP) for all the degrees of freedom of the discretization,

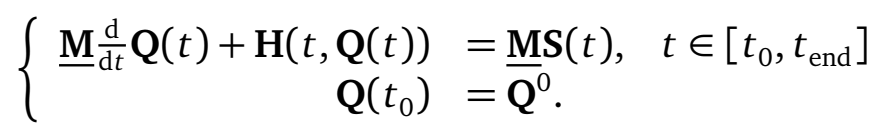

where the initial condition is noted as $\mathbf{Q}\left(t_{0}\right)=\mathbf{Q}^{0}$ (this notation is not to be confused with the polynomial order). Note that $\mathbf{H}$ may depend on $t$ through the boundary condition, and that $\mathbf{S}$ may also depend on $t$ due to external forces. The first equation of the system (3.1) can be rewritten as

$$
\frac{\mathrm{d}}{\mathrm{d} t} \mathbf{Q}(t)=\mathbf{F}(t, \mathbf{Q}(t))
$$

If a steady solution is sought, the term on the left-hand-side can be omitted. This leads to a system of nonlinear equations that may be ill conditioned. As a result, a common way to obtain a steady-state solution is to treat the problem as transient, where the time evolution of $\mathbf{Q}$ is described by (3.2), starting from an arbitrary initial condition. The resulting system is then integrated in time until it converges to a steady solution.

To approximate the time evolution of $\mathbf{Q}$, the time derivative is substituted by a discrete operator (i.e. a time-marching scheme), which in this thesis is noted with 
lowercase deltas,

$$
\frac{\mathrm{d} \mathbf{Q}}{\mathrm{d} t} \leftarrow \frac{\delta \mathbf{Q}}{\delta t},
$$

so that we obtain a completely discrete system from (3.2),

$$
\frac{\delta}{\delta t} \mathbf{Q}(t)=\mathbf{F}(t, \mathbf{Q}(t))
$$

The most straightforward method is to discretize the time derivative with a finite difference, and to evaluate the spatial operator in some appropriate time. By doing that, we might obtain the forward Euler scheme,

$$
\mathbf{Q}^{n+1}=\mathbf{Q}^{n}+\Delta t \mathbf{F}\left(\mathbf{Q}^{n}\right)
$$

and the backward Euler scheme,

$$
\mathbf{Q}^{n+1}=\mathbf{Q}^{n}+\Delta t \mathbf{F}\left(\mathbf{Q}^{n+1}\right)
$$

where $\Delta t$ is called the time-step size and the solution at the time $t_{n}$ is approximated as $\mathbf{Q}\left(t_{n}\right) \approx \mathbf{Q}^{n}$. For the sake of simplicity, the superscript notation is also adopted for the term $\mathbf{F}$, so that $\mathbf{F}\left(t_{n}, \mathbf{Q}^{n}\right)=\mathbf{F}\left(\mathbf{Q}^{n}\right)$.

The Euler schemes are the main exponents of two classification branches for time-integration methods. On the one hand, the forward Euler scheme belongs to the explicit time-integration methods as the solution in a specific time step depends explicitly on the solution in previous time steps (already known). On the other hand, the backward Euler scheme is an implicit time-integration method since the solution in any time step depends implicitly on itself. Hence, a system of nonlinear equations must be solved at every time step to obtain $\mathbf{Q}^{n+1}$.

In what remains of this chapter, a brief review of some explicit and implicit time-integration methods (that will be used in this thesis) and their properties is presented.

\subsection{General Properties}

We are interested in two properties of the time-integration methods: the order order of convergence and the stability characteristics. The former provides information about the accuracy properties of the scheme.

Definition 3.1 (Order of Convergence) A method is considered convergent of order $p$ if some norm of the error at any time step, $\left\|\mathbf{E}^{n}\right\|=\left\|\mathbf{Q}\left(t_{n}\right)-\mathbf{Q}^{n}\right\|$, is proportional to the $p$ power of the time-step size. Using the Bachmann-Landau notation, we say that the norm of the error is $\mathscr{O}\left(\Delta t^{p}\right)$. 
It is easy to show that the Euler schemes are first order accurate $(p=1)[21]$, i.e. the error decreases linearly as the time-step size is reduced.

Note that by the use of the Bachmann-Landau notation, $\Delta t \ll 1$ is supposed. Nonetheless, that may not be the case in some applications. Hence, $\Delta t$ in Definition 3.1 must be understood as a normalized time-step size. In fact, the advantage of using high-order time-stepping methods is that they enable one to increase the time-step size for a given error, so that the term $\mathbf{F}$ must be evaluated a fewer number of times, hence reducing the computational cost. Unfortunately, the time-step size cannot always be increased indiscriminately without affecting the stability of the scheme.

In the numerical solution of partial differential equations, there are many notions of stability. One of the most basic definitions of stability is absolute stability [125], which is, roughly speaking, the ability of a numerical method to not blow up (or crash) nonphysically. In general, a numerical method is considered to be absolutely stable if a selected norm of the numerical solution, or more precisely its error, remains bounded as $t \rightarrow \infty$, if the analytical solution also does [21, 98].

A bounded numerical solution implies that the numerical method does not accumulate rounding or discretization errors unboundedly. Since that is highly dependent on the right-hand-side of equation (3.4), a useful strategy to study the stability properties of a time-marching scheme is to analyze it with the scalar Dahlquist test equation [21],

$$
\frac{\mathrm{d} q}{\mathrm{~d} t}=\lambda q
$$

The analytical solution to (3.7) remains bounded if the real part of $\lambda$ is negative. However, even if $\operatorname{Re}(\lambda)<0$, the boundedness of the solution to the time-discretized version of (3.7),

$$
\frac{\delta}{\delta t} q=\lambda q
$$

depends on the method used to discretize the time derivative and the time-step size, $\Delta t$.

For a specific time-step size, the set of complex numbers $\Delta t \lambda$ for which the method is stable is called the stability region. The shape and size of that region only depends on the time-integration scheme. For the forward Euler scheme, the stability region is a circle of radius 1 centered on $(-1,0)$; whereas for the backward Euler scheme, it is the whole complex plane, except for the circle of radius 1 centered in $(1,0)$.

When the real part of $\lambda$ is negative, the exact solution is bounded and it is desirable that the numerical one is bounded as well. That raises the concept of A-Stability.

Definition 3.2 (A-Stability) A scheme is A-stable if its region of absolute stability contains the left half of the complex plane. 
An A-stable time-marching scheme can take time steps of any size without becoming unstable. It is easy to show [45] that the backward Euler method is A-stable, but the forward Euler is not. In general, explicit methods are not A-stable (although there are some exceptions [205]), while some implicit methods are.

For a time-discretized multivariable equation with linear dependence on constant coefficients,

$$
\frac{\delta \mathbf{q}}{\delta t}=\underline{\mathbf{A}} \mathbf{q}
$$

the stability region applies to the quantities $\Delta t \lambda_{i}$, where the $\lambda_{i}$ are the eigenvalues of the Jacobian matrix, $\underline{\mathbf{A}}$. In the nonlinear case, a typical strategy is to linearize and consider the eigenvalues of the linearized system.

The eigenvalue analysis is generally very revealing, but may not be completely accurate when the Jacobian matrix is highly nonnormal, as in the analysis of upwind conditions for initial boundary value problems [125]. In such cases, the consideration of $\Delta t \lambda_{i}$ and the region of absolute stability imposes a necessary but not sufficient condition for absolute stability.

\subsection{Explicit Time-Integration}

Even though most explicit time-integration methods are not A-stable, they are widely used because they are simple to implement in serial and in parallel: no system of nonlinear equations must be solved for (3.5). In this section, we review the popular explicit Runge-Kutta methods, which will be used throughout this thesis, and discuss the stability properties of general explicit methods.

\subsubsection{Explicit Runge-Kutta Methods}

Explicit Runge-Kutta (RK) methods are the most used and well known time-integration schemes in the high-order DG community since they provide efficient highorder discretizations for a small cost and are readily parallelizable [97]. In any RK method, the solution is obtained as

$$
\mathbf{Q}^{n+1}=\mathbf{Q}^{n}+\Delta t \sum_{i=1}^{s} b_{i} \mathbf{K}_{i}
$$

where $s$ is the number of stages and

$$
\mathbf{K}_{i}=\mathbf{F}\left(t_{n}+\Delta t \sum_{j=1}^{s} c_{j}, \mathbf{Q}^{n}+\Delta t \sum_{j=1}^{s} a_{i j} \mathbf{K}_{j}\right)
$$


The coefficients of the method are usually arranged in the Butcher tableau:

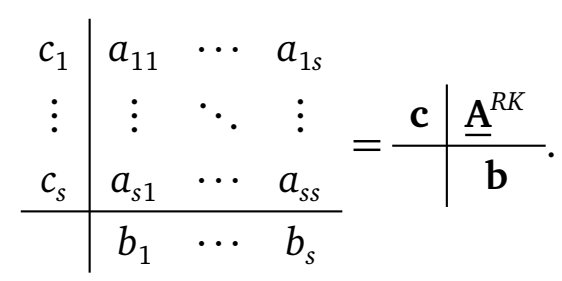

For an RK method to be explicit, the matrix $\underline{\mathbf{A}}^{R K}$ must be strictly lower triangular. For example, the most simple explicit Runge-Kutta method (with one stage, $s=1$ ) is the forward Euler scheme, whose Butcher tableau is

$$
\begin{array}{l|l}
0 & 0 \\
\hline & 1
\end{array}
$$

Another widely used explicit Runge-Kutta method is the fourth order RK, whose coefficients are

$$
\begin{array}{c|cccc}
0 & & & & \\
1 / 2 & 1 / 2 & & & \\
1 / 2 & 0 & 1 / 2 & & \\
1 & 0 & 0 & 1 & \\
\hline & 1 / 6 & 1 / 3 & 1 / 3 & 1 / 6
\end{array} .
$$

Williamson [203] showed that many of the explicit Runge-Kutta schemes can be rearranged to require only two storage locations per variable, as compared to the three that are needed in conventional explicit Runge-Kutta methods. This can be accomplished by reformulating (3.11) as

$$
\mathbf{K}_{i}= \begin{cases}\mathbf{F}\left(t_{n}+\Delta t c_{1}, \mathbf{Q}^{n}\right), & \text { if } i=1, \\ a_{i} \mathbf{K}_{i-1}+\mathbf{F}\left(t_{n}+\Delta t \sum_{j=1}^{i} c_{j}, \mathbf{Q}^{n}+\Delta t \sum_{j=1}^{i-1} b_{j} \mathbf{K}_{j}\right), & \text { otherwise. }\end{cases}
$$

The advantage of low-storage Runge-Kutta methods is more easily appreciated in its algorithm representation (see Algorithm 1), where it is evident that only the vectors $\mathbf{K}$ and $\mathbf{Q}$ must be stored.

Low-storage Runge-Kutta methods are broadly used because of their simplicity and reduced storage requirements. Due to its easy formulation and implementation, the Williamson's low-storage third order Runge-Kutta method (RK3) (see Table 3.1) is widely used in this thesis.

\subsubsection{Stability}

As was discussed in Section 3.1, the location of the eigenvalues, $\lambda_{i}$, in the complex plane depends on the physics and on the spatial discretization that is being used. 


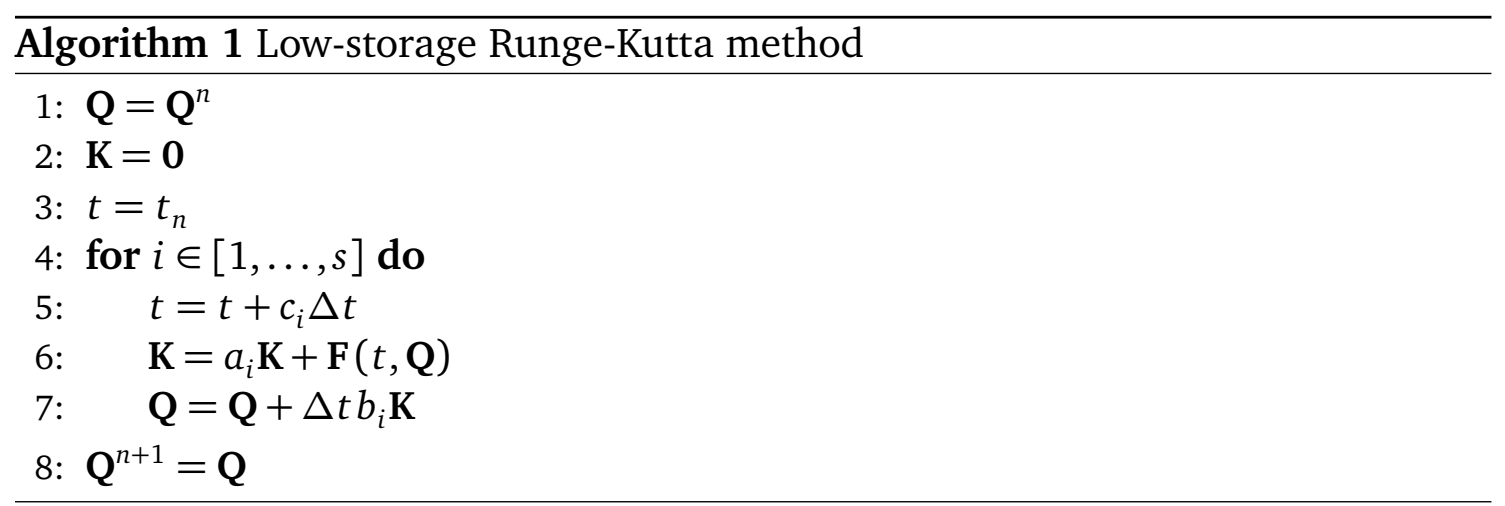

Table 3.1: Williamson's low-storage third order Runge-Kutta coefficients

\begin{tabular}{cccc}
\hline Coefficients $\backslash i=$ & 1 & 2 & 3 \\
\hline$a_{i}$ & 0 & $-5 / 9$ & $-153 / 128$ \\
$b_{i}$ & $1 / 3$ & $15 / 16$ & $8 / 15$ \\
$c_{i}$ & 0 & $1 / 3$ & $3 / 4$ \\
\hline
\end{tabular}

When the resolved physics has advective terms, the time-step size is known to be limited by the Courant-Friedrich-Levy (CFL) condition, which relates the mesh spacing, $h$, with the time-step size. The CFL condition is a necessary, but not always sufficient condition. In particular, for a high-order DG scheme of order $N$ and mesh spacing $h$, the time-step limit is known to behave as $[95,104]$

$$
\Delta t^{a} \leq C^{a}\left(\|\mathscr{S}\| \frac{N^{2}}{h}\right)^{-1}
$$

where $C^{a}$ is a constant that depends on the specific time-integration method that is selected, and $\|\mathscr{S}\|$ is the maximum eigenvalue of the linearized advective flux. In the case of the compressible Euler/Navier-Stokes equations (see Appendix A), $\|\mathscr{S}\|=\|\vec{v}\|^{2}+c$ is the characteristic velocity (with $\vec{v}$ the flow velocity and $c$ the speed of sound).

Similarly, the time-step restriction imposed by the diffusive terms is $[95,104]$

$$
\Delta t^{v} \leq C^{v}\left(\mu \frac{N^{4}}{h^{2}}\right)^{-1},
$$

where, again, $C^{v}$ depends on the specific time-integration scheme, and $\mu$ is the diffusivity (viscosity and thermal conductivity in the case of fluid mechanics). As a result, the maximum allowable time-step size for an advection-diffusion problem is obtained by taking the minimum of both constraints:

$$
\Delta t=\min \left(\Delta t^{a}, \Delta t^{v}\right) .
$$

To not be limited by a fixed time step, most explicit CFD codes use adaptive time-stepping, where this quantity is evaluated at every time step in different re- 
gions of the domain and the most restrictive $\Delta t$ is chosen. One additional possibility to speed up the computations is to use so-called local time-stepping, where each element advances in time independently with its locally computed $\Delta t$. This approach is directly applicable for steady state problems, but it needs additional considerations for time-accurate ones [78, 130].

\subsection{Implicit Time-Integration}

For implicit time-integration methods, the solution at a given time step depends (at least partially) on itself. As a result, a system of linear or nonlinear equations must be solved at every time step, which makes implicit methods much more expensive per time step than explicit methods.

The reason why implicit time-stepping methods are used is that they usually have better stability properties than explicit schemes (see Section 3.2.2) and larger time steps can be taken. This is particularly advantageous when solving stiff problems, where the stability constraint of explicit methods may require a time-step size that is much smaller than the one needed to resolve the physical scales. Examples of such problems are steady-state simulations, problems where boundary layers are present and a very small grid is needed to resolve it accurately, problems with stiff source terms (e.g. in immerse boundary methods), multiphase problems with Lagrangian tracking of parcels, among many others.

This section contains a brief review of the BDF methods, which are the implicit time-integration schemes used in this thesis.

\subsubsection{Backward Differentiation Formulas (BDFs)}

The backward differentiation formulas are the most widespread implicit linear multistep methods and are especially used for the solution of stiff differential equations with high order accuracy. These methods approximate the derivative of a function at a given time step using already computed solutions at previous time steps,

$$
\left.\frac{\mathrm{d} \mathbf{Q}}{\mathrm{d} t}\right|_{t=t_{n}} \approx \sum_{k=0}^{s} a_{k} \mathbf{Q}^{n+1-k}
$$

where $s$ is the order of the approximation and the $a_{k}$ are coefficients that depend on the time step size.

Since the BDFs are implicit time-stepping methods, the nonlinear operator is evaluated with $\mathbf{Q}^{n+1}$. Therefore, inserting (3.19) into (3.2), the BDF approximate solution yields

$$
\mathbf{Q}^{n+1}=\frac{1}{a_{0}}\left(-\sum_{k=1}^{s} a_{k} \mathbf{Q}^{n+1-k}+\mathbf{F}\left(\mathbf{Q}^{n+1}\right)\right)
$$


The BDFs methods can be used with constant or adaptive time-stepping. For constant $\Delta t$, the coefficients are shown in Table 3.2. Note that the $s=1$ scheme is the backward Euler scheme and that the storage of $s$ previous solutions is necessary.

The main drawback of BDF methods is that they are A-stable only up to order $2[22]$.

Table 3.2: Coefficients for BDF up to order 5

\begin{tabular}{ccccccc}
\hline & \multicolumn{7}{c}{ Coefficients $\left(a_{k} \Delta t\right)$} \\
\hline Order $(s) \backslash k=$ & 0 & 1 & 2 & 3 & 4 & 5 \\
\hline 1 & 1 & -1 & & & & \\
2 & $3 / 2$ & -2 & $1 / 2$ & & & \\
3 & $11 / 6$ & -3 & $3 / 2$ & $-1 / 3$ & & \\
4 & $25 / 12$ & -4 & 3 & $-4 / 3$ & $1 / 4$ & \\
5 & $137 / 60$ & -5 & 5 & $-10 / 3$ & $5 / 4$ & $-1 / 5$ \\
\hline
\end{tabular}




\section{Part II \\ Solver Technologies for Time-Marching Schemes}

Summary This part is devoted to the enhancement of the computational efficiency of the solvers for different time-marching schemes. Chapter 4 presents a compilation of well-known solvers for linear and nonlinear systems of equations that are used for implicit and explicit time-integration schemes. In Chapter 5, we analyze strategies to compute the Jacobian matrix of a time-implicit DGSEM, and we derive and implement the DG analytical Jacobian for general nonlinear advection-diffusion conservation laws. Finally, a novel static-condensation method for the Gauss-Lobatto DGSEM is presented in Chapter 6. 

$\Gamma_{\text {Chapter }}$

\section{Background: Solution Techniques for Systems of Equations}

\section{Contents}

4.1 Introduction . . . . . . . . . . . . . . . . . . . 39

4.2 Solving Systems of Nonlinear Equations $\ldots \ldots \ldots \ldots$

4.2 .1 Newton's Method . . . . . . . . . . . . . . . . . . . 40

4.2 .2 Nonlinear Multigrid . . . . . . . . . . . . . . . 41

4.3 Solving Systems of Linear Equations . . . . . . . . . . . 46

4.3 .1 Direct Methods . . . . . . . . . . . . . . . . 46

4.3.2 Splitting Methods . . . . . . . . . . . . . . . . 47

4.3 .3 Krylov Subspace Methods . . . . . . . . . . . . . . . . 48

4.3 .4 Linear Multigrid . . . . . . . . . . . . . . . . . 51

\subsection{Introduction}

In many cases, the DG discretization of a nonlinear PDE leads to a system of nonlinear equations that must be solved to obtain the sought solution. As mentioned in Chapter 3, that is the case for steady non-time-marching methods and most implicit time-integration schemes (for Rosenbrock-type RK schemes, where a linear system is obtained instead). However, as will be evident in following sections, explicit methods can also be formulated in such a way that a system of nonlinear equations arises.

The efficient solution of these nonlinear systems is fundamental for the performance of a CFD code. Therefore, this chapter is devoted to the analysis of several methods to solve systems of linear and nonlinear equations that are broadly used 
in the the literature. We do not intend to make an exhaustive review here, but to introduce important concepts that will be useful in the rest of this thesis. Note that the spatial discretization for these methods is fixed and the main idea here is to reduce the computational cost to obtain a solution by the choice of the solution strategy.

This chapter is organized as follows. Section 4.2 presents an overview of two methods that are broadly used to solve systems of nonlinear equations: the well known Newton's method and the nonlinear multigrid scheme. Often, these techniques lead to the appearance of successive systems of linear equations that must be solved to obtain an approximate solution. Afterwards, Section 4.3 presents some techniques to solve systems of linear equations that are used in the rest of this thesis.

\subsection{Solving Systems of Nonlinear Equations}

Since many interesting applications require one to solve a system of nonlinear equations, in this section we introduce two broadly used methods that accomplish that objective: the Newton's method and the nonlinear multigrid scheme.

\subsubsection{Newton's Method}

The Newton's method and its variants are by far the most extensively used methods to solve systems of nonlinear equations. The standard Newton's method acts on a nonlinear system of the form,

$$
\mathbf{R}(\mathbf{Q})=\mathbf{S} \text {. }
$$

$\mathbf{R}(\mathbf{Q})$ is expanded using a Taylor series, so that (4.1) is rewritten as

$$
\begin{aligned}
\mathbf{R}(\mathbf{Q}) & =\mathbf{R}\left(\mathbf{Q}_{0}\right)+\left.\frac{\partial \mathbf{R}}{\partial \mathbf{Q}}\right|_{\mathbf{Q}_{0}} \Delta \mathbf{Q}+\mathscr{O}\left(\|\Delta \mathbf{Q}\|^{2}\right)=\mathbf{S} \\
& \approx \mathbf{R}\left(\mathbf{Q}_{0}\right)+\underline{\mathbf{A}}\left(\mathbf{Q}_{0}\right) \Delta \mathbf{Q},
\end{aligned}
$$

where $\Delta \mathbf{Q}=\mathbf{Q}-\mathbf{Q}_{0}$ is the solution update, and $\underline{\mathbf{A}}\left(\mathbf{Q}_{0}\right)$ is the Jacobian matrix of the nonlinear operator evaluated at $\mathbf{Q}_{0}$. It is possible to reorganize (4.2) to get the 2-stage process:

1. Solve the linearized problem: $\underline{\mathbf{A}}\left(\mathbf{Q}_{0}\right) \Delta \mathbf{Q}=\mathbf{S}-\mathbf{R}\left(\mathbf{Q}_{0}\right)$.

2. Let $\mathbf{Q}=\mathbf{Q}_{0}+\Delta \mathbf{Q}$.

Step 1 requires the solution of a system of linear equations. This can be done with a variety of direct or iterative linear solvers (see Section 4.3). Furthermore, it is important to notice that, since the Taylor series is truncated and only linear terms are considered, the 2 -stage procedure must be solved iteratively to get an 
accurate solution, as seen in Algorithm 2. Generally, the solution is considered to be good enough when the predicted correction, $\Delta \mathbf{Q}$, is small. At that point, the iterative solution procedure can be stopped.

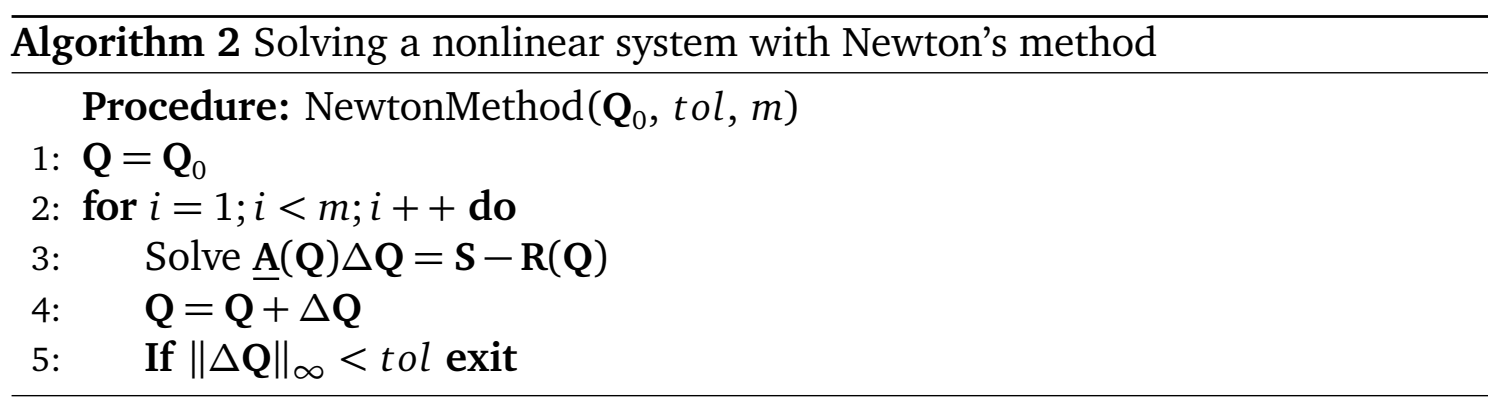

It has been proved that the standard Newton's method, which computes the matrix in every iteration, is second order convergent [21]. However, the computation of the Jacobian matrix may be very expensive. Therefore, in practice it is useful to use simplified Newton methods, where the Jacobian matrix is not computed in every iteration, but it is kept unchanged (frozen) for several iterations, or even time steps, whereas the RHS is updated in every iteration. This slightly deteriorates the order of convergence, but improves the overall computational performance of the method. Another alternative is to use quasi-Newton methods (also called methods of Newton type) [55], which rely on the same formulation, but instead of the exact Jacobian, another matrix, which approximates the Jacobian, is used.

The choice of the initial guess is very relevant because the Newton's method is locally convergent. Usually, an adequate initial guess is the solution in the previous time step.

\subsubsection{Nonlinear Multigrid}

Multigrid methods were first proposed by Brandt [32], who discovered that iterative methods eliminate high-frequency components of the error quickly (i.e. to smooth the error), but they fail to eliminate low-frequency components efficiently. As a consequence, the term smoothers was coined for general iterative methods in the multigrid community. Here, a smoother can be anything from a Newton's method, to a time-marching scheme, or combinations and variations of those.

Brandt proposed to use a limited number of iterations of the smoother on the original mesh to only remove the high-frequency modes of the error and then transfer the problem to coarser meshes (with a fewer number of elements) to eliminate the low-frequency modes. His approach is known as $h$-multigrid, and has been used extensively with low-order methods such as traditional Finite Difference and Finite Volume schemes [101, 123, 198].

Zang, Wong and Hussaini [206], Craig and Zienkiewicz [51], and Rønquist and Patera [173] were the first authors working on high-order methods that proposed the use of the polynomial order, usually $p$, to define the levels of a multigrid 
scheme. After these initial works, the use of multilevel methods spread in the highorder community; initially as $p$-multigrid methods [13, 68, 131, 132, 201] and more recently as $h p$-multigrid methods [28, 149, 151, 185, 200]. Most of these implementations use modal hierarchical shape functions [68, 151, 185, 201], and only a small number of publications focus on nodal-based shape functions $[13,68]$.

Two types of multilevel methods can be found in the literature: linear and nonlinear multigrid methods. The former is de facto a linear solver and is discussed in Section 4.3.4. The latter, also known as Full Approximation Scheme (FAS), applies multigrid directly to the set of nonlinear equations. A comparison of linear and nonlinear multigrid methods for DG discretizations can be found in [151].

In this section we shortly describe the Full Approximation Storage (FAS) nonlinear $p$-multigrid algorithm. $h$-Multigrid relies on the same concepts $[33,144,151$, 201]. The starting point is the same system of nonlinear equations (4.1), but let us now explicitly write the polynomial order $(P)$ :

$$
\mathbf{R}^{P}\left(\mathbf{Q}^{P}\right)=\mathbf{S}^{P},
$$

where $P=\left(P_{1}, P_{2}, P_{3}\right)$ can be different in each element and each coordinate direction. After some smoothing sweeps, the nonlinear residual equation,

$$
\mathbf{R}^{P}\left(\tilde{\mathbf{Q}}^{P}\right)=\mathbf{S}^{P}+\tilde{\mathfrak{R}}^{P},
$$

holds, where $\tilde{\mathbf{Q}}^{P}$ is the approximated solution and $\tilde{\mathfrak{R}}^{P}$ is known as the nonlinear residual.

Note that (4.3) can refer to a time-implicit discretization of an unsteady PDE or to a steady-state form of $(3.2)(\partial \mathbf{q} / \partial t=0)$. In the latter case, the nonlinear DG operator (see Chapter 2) is

$$
\mathbf{R}^{P}\left(\mathbf{Q}^{P}\right)=\underline{\mathbf{M}}_{P}^{-1} \mathbf{H}^{P}\left(\mathbf{Q}^{P}\right),
$$

and the smoothing procedure can be the iterative Newton's method or a timemarching scheme. For instance, if an explicit time-integration method is used, there is no need to solve linear systems in the smoothing procedure.

Using (4.3), (4.4) can be rewritten as

$$
\begin{aligned}
\mathbf{R}^{P}\left(\tilde{\mathbf{Q}}^{P}\right)-\mathbf{R}^{P}\left(\mathbf{Q}^{P}\right) & =\tilde{\mathfrak{R}}^{P}, \\
\mathbf{R}^{P}\left(\tilde{\mathbf{Q}}^{P}\right)-\mathbf{R}^{P}\left(\tilde{\mathbf{Q}}^{P}+\boldsymbol{\epsilon}_{i t}^{P}\right) & =\tilde{\mathfrak{R}}^{P},
\end{aligned}
$$

where $\epsilon_{i t}^{P}$ is the so called iteration error on the mesh $P$, the difference between the solution of order $P$ and the approximation in the current iteration. The standard two-level FAS $p$-multigrid scheme consists in transferring (4.6) to a lower polynomial discretization of order $N=P-\Delta N$ (coarser grid), and using additional smoothing sweeps there. On the lower-order grid, the smoother now targets frequencies of the error lower than the ones removed on the finer grid. Therefore, solving the residual equation on the coarse grid,

$$
\mathbf{R}^{N}\left(\tilde{\mathbf{Q}}_{0}^{N}\right)-\mathbf{R}^{N}\left(\mathbf{Q}^{N}\right)=\tilde{\mathfrak{R}}^{N},
$$


for $\mathbf{Q}^{N}$, leads to an improved low frequency approximation of the fine grid problem, if $\tilde{\mathbf{Q}}_{0}^{N}$ and $\tilde{\mathfrak{R}}^{N}$ are transferred (interpolated or projected) from the fine grid:

$$
\begin{aligned}
\tilde{\mathbf{Q}}_{0}^{N} & =\underline{\mathbf{I}}_{P}^{N} \tilde{\mathbf{Q}}^{P}, \\
\tilde{\mathfrak{R}}^{N} & =\underline{\mathbf{I}}_{P}^{N} \tilde{\mathfrak{R}}^{P} .
\end{aligned}
$$

In (4.9) and (4.10), $\underline{\mathbf{I}}_{P}^{N}$ is the restriction operator, an $L_{2}$ projection to the lower polynomial order. Note that no distinction is made between the solution and residual transfer operators since in this work both the solution and the residual are spanned in the same polynomial space. It is also important to remark that the $L_{2}$ projection guarantees the conservation of the transferred quantities and therefore it is preferred in this work over simple interpolation.

In (4.8), $\mathbf{R}^{N}\left(\tilde{\mathbf{Q}}_{0}\right)$ and $\tilde{\mathfrak{R}}^{N}$ are fixed terms that ensure that the solution on the coarse grid approximates the solution of the fine-grid problem. Moreover, note that $\tilde{\mathbf{Q}}_{0}$ is a good choice for the initial guess of $\tilde{\mathbf{Q}}$ (therefore the subindex 0). Taking all these into account, the coarse-grid nonlinear problem,

$$
\mathbf{R}^{N}\left(\mathbf{Q}^{N}\right)=\mathbf{S}^{N}
$$

holds, where $S^{N}$ is an artificial source term that can be obtained combining (4.8), (4.9) and (4.10):

$$
\mathbf{S}^{N}=\mathbf{R}^{N}\left(\underline{\mathbf{I}}_{P}^{N} \tilde{\mathbf{Q}}^{P}\right)-\underline{\mathbf{I}}_{P}^{N} \tilde{\mathfrak{R}}^{P},
$$

which in steady-state problems, according to (4.5), is the same as

$$
\mathbf{S}^{N}=\underline{\mathbf{M}}^{-1} \mathbf{H}^{N}\left(\underline{\mathbf{I}}_{P}^{N} \tilde{\mathbf{Q}}^{P}\right)-\underline{\mathbf{I}}_{P}^{N} \tilde{\mathfrak{R}}^{P} .
$$

After solving (4.11) for $\mathbf{Q}^{N}$, we obtain a low frequency approximation of the iteration error,

$$
\boldsymbol{\epsilon}_{i t}^{N}=\mathbf{Q}^{N}-\tilde{\mathbf{Q}}_{0}^{N},
$$

which is then used to correct the solution on the fine grid:

$$
\tilde{\mathbf{Q}}^{P}=\tilde{\mathbf{Q}}^{P}+\underline{\mathbf{I}}_{N}^{P} \boldsymbol{\epsilon}_{i t}^{N} \text {. }
$$

The two-level process described above can be generalized to a multilevel FAS V-Cycle and coded efficiently as a recursive procedure, as depicted in Algorithm 3 . Note that the superindex $c$ now denotes the next coarser multigrid level, that the fine-mesh superindexes have been dropped for readability, and that instead of completely solving the coarse-grid problem (4.11), we only perform some coarsegrid smoothing cycles. The multigrid V-cycle has $N_{M G}$ levels, where level $=1$ is the coarsest (lowest polynomial order) and level $=N_{M G}$ is the finest (highest polynomial order).

In this thesis, we always use $\Delta N=P-N=1$ as the polynomial order reduction when coarsening since the idea is to act on all possible frequencies. Therefore, the number of multigrid levels corresponds to the maximum polynomial order of the mesh. Moreover, since in the DGSEM the geometry approximation order must be at most the solution approximation order, $M \leq N$ (see Section 2.2.1), the lowest multigrid level is set in this thesis to $N=1$. 


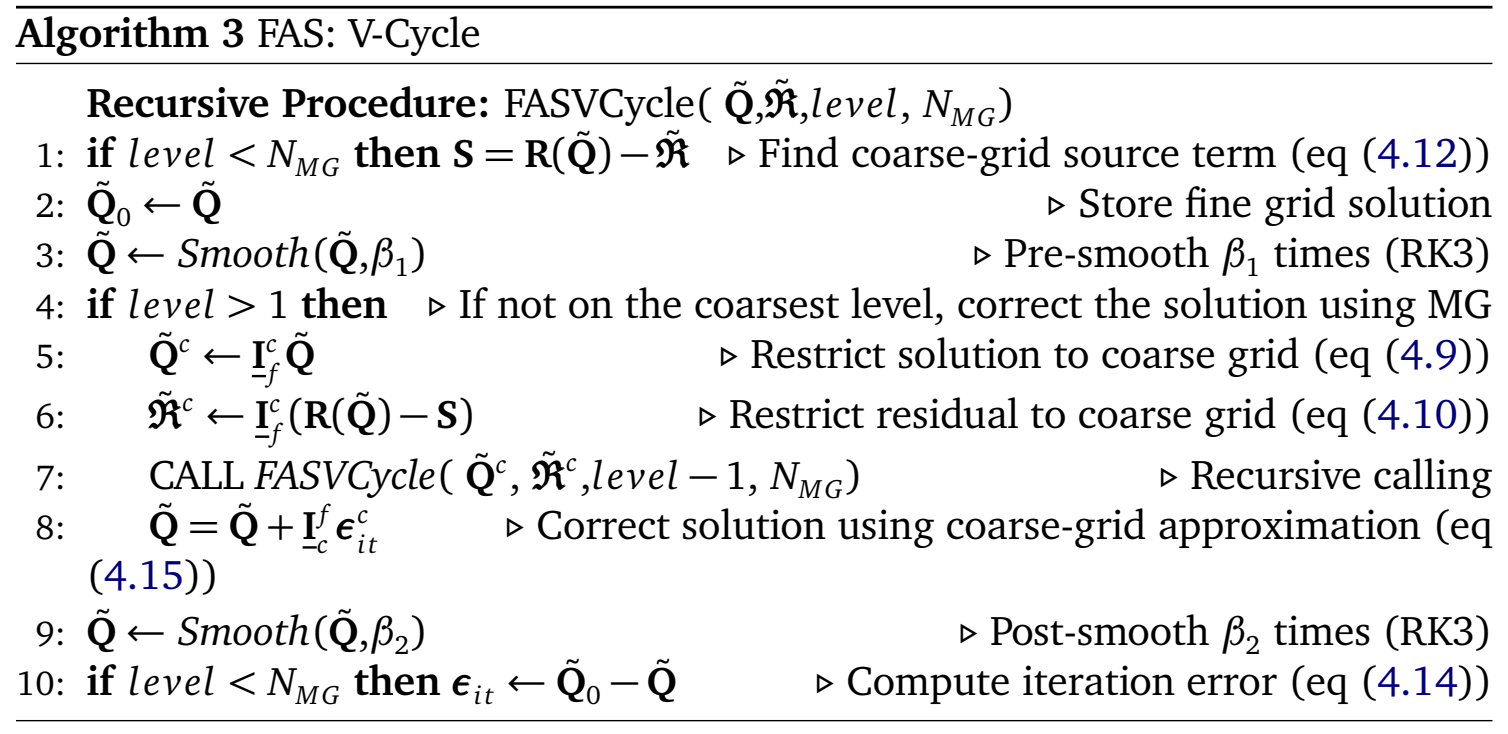

\subsubsection{Multigrid Cycling Strategy}

The typical cycling strategy used for $h$ - and $h p$-multigrid implementations is to perform repeated V-cycles [28, 68, 131, 132, 149, 151, 173, 185, 200] (see Figure 4.1(a)). Some authors [144, 185] make use of V or W saw-tooth cycles (without post-smoothing). This technique is well-suited for modal discretizations since the solution correction (4.14) is injected in the low-order coefficients of the fine-grid discretization after coarse-grid smoothing. However, in the nodal discretizations of DGSEM (used in this thesis), the coarse-grid smoothed solution can excite highfrequency modes of the fine-grid discretization after the interpolation to the fine grid. In consequence, we find that post-smoothing is required.

The V-cycling strategy can be very sensitive to the initial condition. To get an appropriate initial condition in the high-order discretization, the mainstream alternative is to employ a Full Multigrid (FMG) cycle (see Figure 4.1(b)) at the beginning of the simulation. In this cycling strategy, some iterations are taken with the lowest polynomial order (coarsest multigrid level) and the approximated solution is projected to the next (finer) approximation. There, a number of V-cycles are taken before increasing the polynomial order again. This procedure is repeated until the finest multigrid level is reached.

In the FMG cycle, the number of iterations in every level can be fixed [151, $185]$ or can be tuned using a residual-based approach $[13,68]$. In this work, we use a residual-based approach where multiple V-cycle repetitions are taken at each level until a predefined residual is reached; when that happens, the approximation level is raised. Such a cycling strategy can be easily implemented using a recursive routine, as shown in Algorithm 4. 


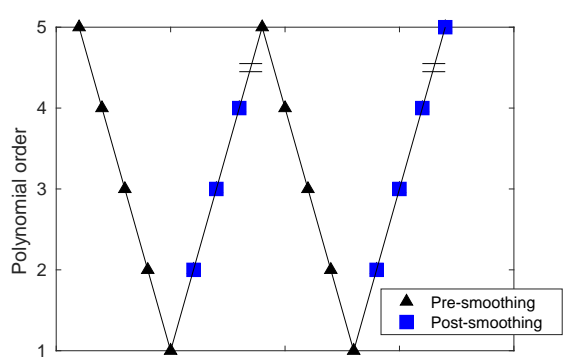

(a) V-cycle.

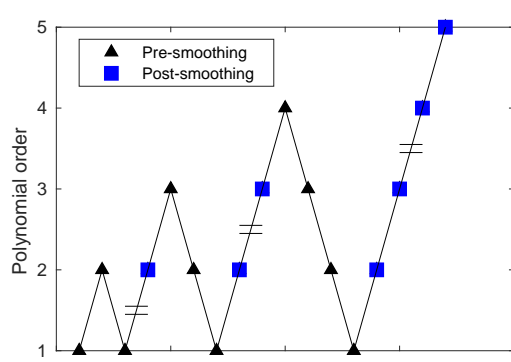

(b) FMG-cycle for getting an appropriate initial condition.

Figure 4.1: FMG- and V-cycing strategies. Equal signs represent the continuation of the V-cycling process until reaching the desired residual.

\subsubsection{Designing the smoothing}

In general, the number of pre-smoothing sweeps $\left(\beta_{1}\right.$ in Algorithm 3 ) must be high enough to ensure that the high-frequency modes of the error have been smoothed out, so that an inexact $L_{2}$ restriction cannot introduce noise into lower multigrid levels. Likewise, the number of post-smoothing sweeps $\left(\beta_{2}\right)$ must be high enough to guarantee that mid-frequency modes of the error do not develop into higherorder discretizations. These mid-frequency modes of the error can be excited by the inexact $L_{2}$ prolongation of the solution that was smoothed in a lower multigrid level.

A common practice is to set a fixed number of pre- and post-smoothing sweeps $[13,68,94,151,185]$. It is also common to tune the number of smoothing sweeps using a residual-based strategy that checks if enough smoothing was performed $[13,32,68]$. When very high polynomial orders and anisotropic nonconforming discretizations are used, some stages of the simulation can be very sensitive to insufficient smoothing (e.g. at the beginning of the simulation or after an adaptation stage). With that in mind, we propose two residual-based strategies for tuning the number of smoothing sweeps:

1. Pre-smoothing: After every $\beta_{1}^{0}$ sweeps (fixed number), the residual in the next (coarser) discretization is checked. If $\left\|\tilde{\mathfrak{R}}^{P}\right\|_{\infty}<\eta\left\|\tilde{\mathfrak{R}}^{N}\right\|_{\infty}$, the presmoothing is stopped; otherwise, $\beta_{1}^{0}$ additional sweeps are performed. This strategy is a modification of the residual-based approach that some authors employ in FMG cycles to check if the coarse level smoothing is enough [13, 68].

2. Post-smoothing: The norm of the residual after the post-smoothing must be at least as low as it was after the pre-smoothing, $\left\|\tilde{\mathfrak{R}}_{\text {post }}^{N}\right\|_{\infty} \leq\left\|\tilde{\mathfrak{R}}_{\text {pre }}^{N}\right\|_{\infty}$. This 


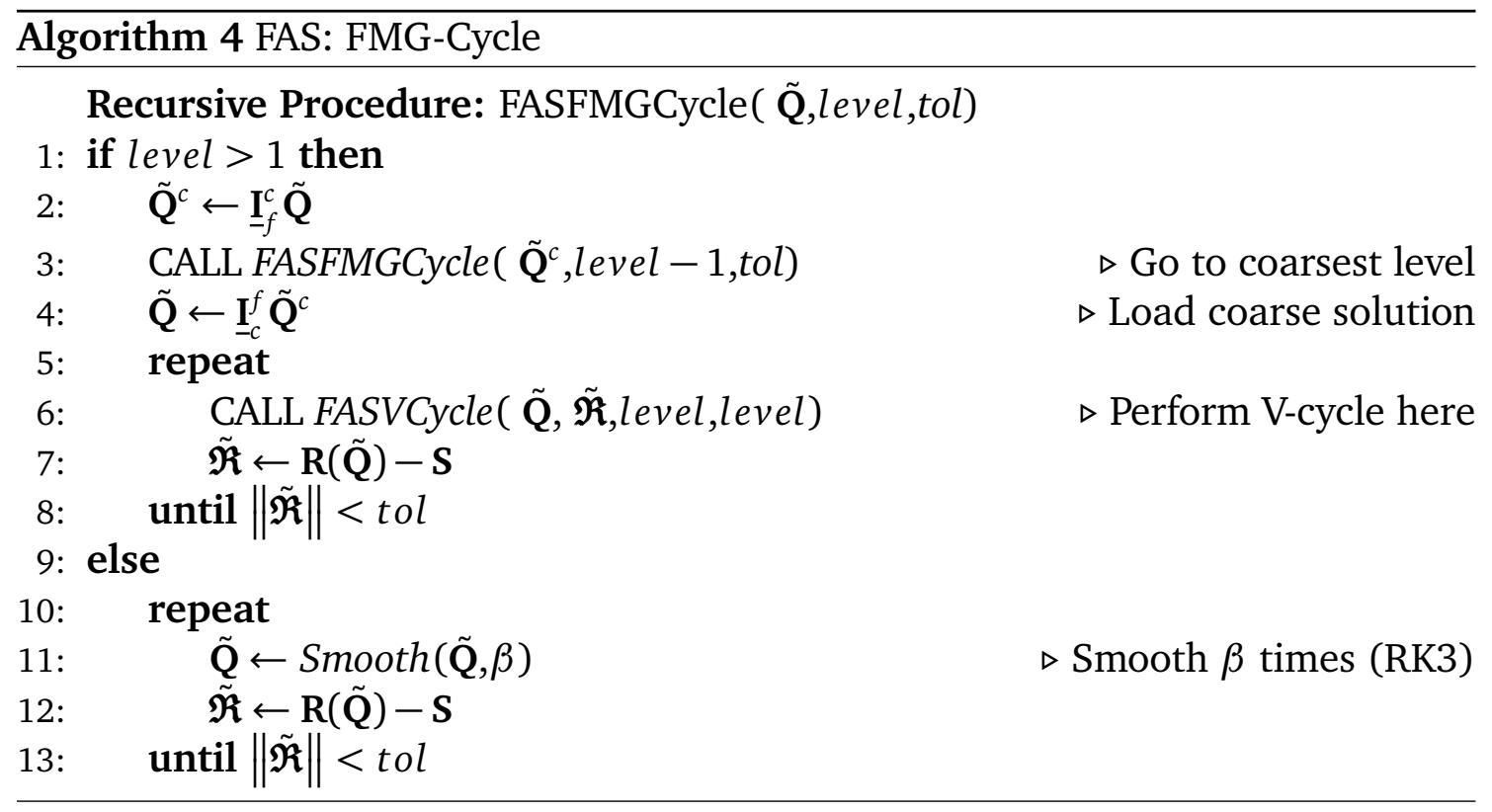

condition is checked every $\beta_{2}^{0}$ sweeps and the post-smoothing loop is exited when fulfilled. This way, we guarantee that most of the high-frequency errors that could be excited during coarse smoothing are eliminated.

\subsection{Solving Systems of Linear Equations}

When the Newton's method is used, the original nonlinear problem is reduced to solving successive systems of linear equations of the form

$$
\underline{\mathbf{A X}}=\mathbf{B}, \quad \underline{\mathbf{A}} \in \mathbb{R}^{n \times n} .
$$

There are multiple solving techniques available in the literature, being the most prominent ones the direct methods, the classical iterative methods, the Krylov subspace methods and the linear multigrid methods. A brief review these is given in this section. For further details, refer to [32, 33, 182].

\subsubsection{Direct Methods}

Direct methods are the most reliable methods for solving linear systems since they obtain an exact solution to the problem to within rounding/conditioning errors. The most well known direct method is Gaussian elimination, which reduces the system to a triangular one using row operations. Once the system is triangular, the solution can be found via backward substitution. Gaussian elimination is considered to be a very expensive method since it requires $\mathscr{O}\left(2 n^{3} / 3\right)$ floating point operations. Many other alternatives are available in the literature, such as the Cholesky 
decomposition (for Hermitian, positive-definite matrices), the QR decomposition and the LU decomposition (both for general nonsymmetric systems). In next section, LU decomposition is briefly described, as it is the most used and efficient method for real nonsymmetric systems.

\subsubsection{LU Decomposition}

The idea of LU decomposition is to factorize the system matrix as the product of non-singular lower- and upper-diagonal matrices $L$ and $U$,

$$
\underline{\mathbf{A}}=\underline{\mathbf{L U}} \text {. }
$$

Depending on the structure of matriz $\underline{\mathbf{A}}$, this is not always possible. However, it has been shown [194] that a proper reordering of the matrix by rows or columns, also called permutation, is sufficient to enable the LU factorization of general nonsingular matrices,

$$
\underline{\mathbf{P A}}=\underline{\mathrm{LU}},
$$

where $\underline{\mathbf{P}}$ is known as the permutation matrix.

Once the matrix is factorized, the linear system can be solved in two steps:

1. Solve the linear system $\underline{\mathbf{L Y}}=\underline{\mathbf{P B}}$ for $\mathbf{Y}$.

2. Solve the linear system $\underline{\mathbf{U X}}=\mathbf{Y}$ for $\mathbf{X}$.

Since the matrices $\underline{\underline{L}}$ and $\underline{\mathrm{U}}$ are triangular, both linear solves can be done by backward and forward substitution. Therefore, the solving process is very cheap, requiring approximately $\mathscr{O}\left(n^{2} / 2\right)$ operations. The expensive part is the factorization of the matrix, which requires around $\mathscr{O}\left(n^{3} / 3\right)$ operations. As a result, the LU factorization is a feasible possibility when the factorized matrix can be used for several linear solves with different right-hand-sides, and there is enough storage for $\underline{\mathbf{L}}, \underline{\mathbf{U}}, \underline{\mathbf{A}}$ and the variables needed to factorize the original system.

There are a number of sparse direct LU solvers available that take advantage of the sparsity of the matrix. In those solvers, an early preordering is applied to reduce the fill-in of the factorized matrix [182]. Significant progress has been made in recent decades, and very robust packages have been developed, such as PARDISO [52, 117, 197], SuperLU [127, 128] or UMFPACK [86].

\subsubsection{Splitting Methods}

The splitting methods are classical iterative methods, where the main idea is to obtain better approximations to the solution of the linear system in successive iterations. In these methods, the matrix $\underline{\mathbf{A}}$ is split into an implicit matrix, $\underline{\mathbf{P}}$, and an explicit matrix, $\underline{\mathbf{N}}$

$$
\underline{\mathbf{A}}=\underline{\mathbf{P}}-\underline{\mathbf{N}}
$$


and the iterative process is defined as,

$$
\underline{\mathbf{P Q}}^{k+1}=\underline{\mathbf{N Q}}^{k}+\mathbf{B},
$$

where the superindex, $k$, here denotes the iteration number. Note that the original system is transformed into another that only needs the inversion of $\underline{\mathbf{P}}$. In fact, the solution in the iteration $k+1$ is

$$
\mathbf{Q}^{k+1}=\mathbf{Q}^{k}+\underline{\mathbf{P}}^{-1} \mathbf{R}^{k}
$$

for $\mathbf{R}^{k}=\mathbf{B}-\underline{\mathbf{A Q}}^{k}$.

Some of the most common splitting methods are:

- Jacobi: $\underline{\mathbf{P}}$ is a diagonal matrix containing the diagonal entries of $\underline{\mathbf{A}}$.

- Gauss-Seidel: $\underline{\mathbf{P}}$ is a lower triangular matrix containing the lower triangular entries of $\underline{\mathbf{A}}$.

- Block Jacobi (also known as Element Jacobi): $\underline{\mathbf{P}}$ is the matrix containing the block diagonal terms of $\underline{\mathbf{A}}$. In this case, the computation can be expressed for each $i$ block of the solution as a function of the blocks of the Jacobian, $\underline{\mathbf{A}}_{i j}$,

$$
\underline{\mathbf{A}}_{i i} \mathbf{Q}_{i}^{k+1}=\underline{\mathbf{B}}_{i}-\sum_{\substack{j=1 \\ j \neq i}}^{K} \underline{\mathbf{A}}_{i j} \mathbf{Q}_{j}^{k}, i=1,2, \cdots, K,
$$

where $K$ is the number of diagonal blocks.

The number of operations needed to converge the solution of the linear system depends on the spectrum of the system matrix, $\underline{\mathbf{A}}$, and on how well $\underline{\mathbf{P}}$ approximates it. In general, splitting methods are only linearly convergent with a constant near one [21], which makes them not competitive against direct or Newton Krylov methods. In spite of that, splitting methods are important as preconditioners and smoothers for Krylov subspace methods (Section 4.3.3) and multigrid methods (Section 4.3.4).

\subsubsection{Krylov Subspace Methods}

Krylov subspace methods are iterative methods that search for the solution of the linear system (4.16) in the vector space

$$
\mathbf{X}_{0}+\operatorname{span}\left\{\mathbf{R}_{0}, \underline{\mathbf{A}} \mathbf{R}_{0}, \underline{\mathbf{A}}^{2} \mathbf{R}_{0}, \ldots, \underline{\mathbf{A}}^{m-1}\right\}=\mathbf{X}_{0}+\mathscr{K}_{m}\left(\underline{\mathrm{A}}, \mathbf{B}, \mathbf{X}_{0}\right),
$$

where $\mathbf{X}_{0}$ is the initial guess and $\mathscr{K}_{m}$ is called the $m^{\text {th }}$ Krylov subspace of the linear system. Note that $m$ is at most the size of the system, $n$, but depending on the method, acceptable solutions can be found with $m \ll n$. 
To look for the solution in $\mathscr{K}_{m}$, it is frequently desirable to work with an orthonormal basis of it. The Arnoldi, the Lanczos and the bi-Lanczos algorithms are well known and frequently used methods to construct an orthonormal basis for Krylov subspaces [58].

There are multiple Krylov subspace methods available in the literature that differ in the way the Krylov subspace is constructed, the kinds of linear systems that are targeted for solution (e.g. symmetric or or not, complex or real), the criteria that are taken into account to obtain the solution out of the Krylov subspace (projection or residual minimization), among other features. Each of the available Krylov subspace methods offers advantages and disadvantages depending on the problem studied.

In this section, we review the generalized minimal residual method (GMRES), since it is a suitable choice for the kinds of problems considered in this thesis (real nonsymmetric matrices). For a more detailed overview of the methods, we refer the reader to $[58,182]$.

\subsubsection{The Generalized Minimal Residual Method (GMRES)}

The generalized minimal residual method computes an orthogonal basis of the Krylov subspace using the method of Arnoldi [7], and then uses that basis to look for a solution of the linear problem in the subspace $\mathbf{X}_{0}+\mathscr{K}_{m}$, so that the final residual of the problem,

$$
J(\mathbf{X})=\|\mathbf{B}-\underline{\mathbf{A X}}\|_{2}
$$

is minimized.

The Arnoli method obtains an orthonormal basis for the $m^{\text {th }}$ Krylov subspace by computing one vector at a time, orthogonalizing it against the previously obtained vectors of the basis and normalizing it. Therefore, the process of obtaining a new vector of the orthonormal basis, $\mathbf{V}_{j+1}$, is

$$
\begin{array}{ll}
\mathbf{V}_{j+1}=\underline{\mathbf{A V}}_{j} & \begin{array}{l}
\text { (compute an initial approximation), } \\
\mathbf{V}_{j+1}=\mathbf{V}_{j+1}-\sum_{i=1}^{j}\left(\mathbf{V}_{j+1} \cdot \mathbf{V}_{i}\right) \mathbf{V}_{i}
\end{array} \\
\mathbf{V}_{j+1}=\mathbf{V}_{j+1} /\left\|\mathbf{V}_{j+1}\right\|_{2} & \begin{array}{l}
\text { (orthogonalize against previously obtained } \\
\text { (normalize to get a new vector of the basis). }
\end{array}
\end{array}
$$

Obviously, the $m^{\text {th }}$ Krylov subspace is spanned by $m$ orhonormal vectors. However, in many cases an adequate solution can be obtained as a linear combination of $k<m$ vectors. Therefore, in the GMRES method, every time a new orthonormal vector is computed, the algorithm checks if the vectors computed so far are enough to obtain a solution for a given tolerance. If so, the solution is recovered as

$$
\mathbf{X}=\mathbf{X}_{0}+\sum_{i=1}^{k} \alpha_{i} \mathbf{V}_{i}
$$


where the values of $\alpha_{i}$ are computed by solving a minimization problem, so that $J(\mathrm{X})(4.24)$ is minimized. The process that has been explained so far is summarized in Algorithm 5 (for $\underline{\mathbf{P}}=\underline{\mathbf{I}}_{n}$, the identity matrix of size $n \times n$ ).

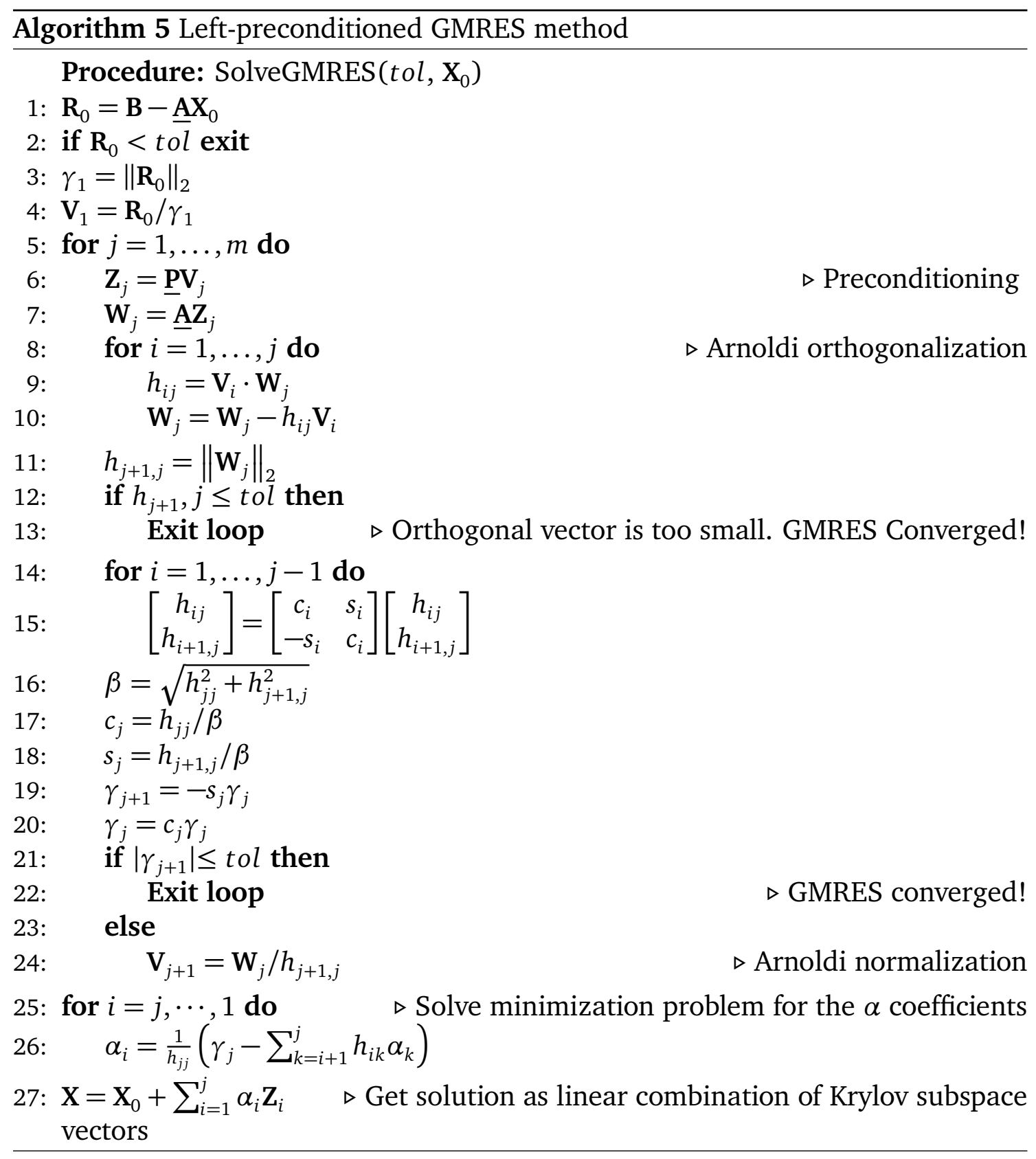

The convergence rate of a Krylov subspace method depends on the matrix $\underline{\mathbf{A}}$. If the matrix is ill conditioned, the GMRES algorithm may need many steps to converge. Unfortunately, the discretization of nonlinear PDEs usually yields very ill conditioned matrices. A widely used strategy is to use preconditioning to convert the original linear system to another that is simpler to solve. Left and right precon- 
ditioning methods are broadly used. The former rewrites the linear system as

$$
\underline{\mathbf{P A X}}=\underline{\mathbf{P B}} .
$$

Right preconditioning converts the system into

$$
\underline{\mathbf{A P X}^{P}}=\mathbf{B},
$$

so that a solution for $\mathbf{X}^{P}$ is sought, which can then be used to recover the solution to the original problem as $\mathbf{X}=\underline{\mathbf{P}} \mathbf{X}^{P}$.

The ideal preconditioner is $\underline{\mathbf{P}}=\underline{\mathbf{A}}^{-1}$ since, in that case, the preconditioned system turns out to have an identity as the system matrix. Hence, the solution is obtained in only one iteration. Since we are interested in problems where $\underline{A}^{-1}$ is not easy to obtain, an approximation is needed. Popular alternatives are based on the splitting methods of Section 4.3.2, on incomplete factorizations, or on multigrid strategies. For a review of classical preconditioning methods refer to [182] and for specific preconditioning for DG methods refer to [21]. Algorithm 5 is the left-preconditioned GMRES method.

The amount of storage that is required for the GMRES method scales rapidly with $m$, as also does the number of operations that are needed for the Arnoldi orthogonalization. An often used remedy is to limit $m$ to a $l o w^{1}$ value and restart the method every $m$ iterations if no solution is ready yet. In that case, the latest approximation of $\mathbf{X}$ is used as the initial guess. This method is called GMRES $(m)$, or restarted GMRES. Depending on the problem, the restarted GMRES can perform better or worse than the original GMRES method [21, 182].

As a final remark, note that the unpreconditioned GMRES method, as any other Krylov subspace method, only needs the matrix action on a vector, $\underline{A}$. This will be shown to be advantageous when formulating Jacobian-free methods in the next chapter. The preconditioned GMRES method, however, needs a way to compute the preconditioner action, $\underline{\mathbf{P V}}$, besides the matrix action.

Very efficient implementations of Krylov subspace methods can be found in the PETSc library [1] and the HYPRE library [60].

\subsubsection{Linear Multigrid}

Iterative methods have good smoothing properties, i.e. when applied to a linear system, they tend to eliminate high frequency errors very fast, but take a long time to eliminate low frequency errors. Therefore, they improve the solution approximation very quickly in the first iterations, but then get very slow when the error is mainly composed by low frequencies. To overcome this issue, multigrid methods can also be applied to linear systems.

In the same way as for nonlinear multigrid methods, when the approximate solution is transferred to a coarser mesh (grid), the non-oscillatory components of

\footnotetext{
${ }^{1}$ Typically, $30<m<60$.
} 
the error become oscillatory, so that the application of iterative methods is again effective. Given the linear system (4.16), an approximation to the solution obtained after some sweeps of an iterative method, $\tilde{\mathbf{Q}}$, has an associated residual,

$$
\underline{\mathrm{A}} \mathbf{Q}-\underline{\mathrm{A}} \mathbf{Q}=\mathbf{R},
$$

and an associated iteration error, $\mathbf{E}=\mathbf{Q}-\tilde{\mathbf{Q}}$. Therefore, the residual equation can be rewritten as

$$
\underline{\mathrm{AE}}=\mathbf{R} .
$$

The basic linear multigrid V-Cycle has several similarities with the nonlinear multigrid version. The main difference is that the coarse-grid smoothing works directly on the iteration error because the problem is linear, as seen in (4.29). The procedure is summarized in Algorithm 6, where again, the superscript $c$ indicates the quantities in the next (coarser) grid and the superscript of the current level is dropped for readability.

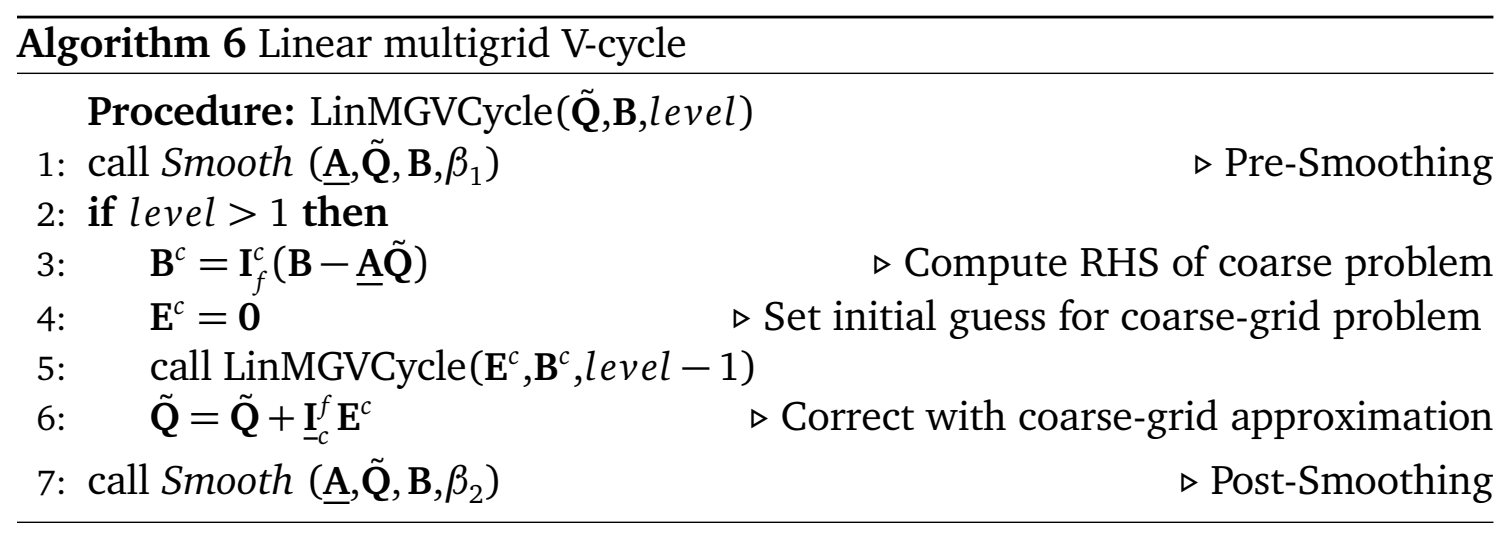

In Algorithm 6, $\underline{\mathbf{I}}_{f}^{c}$ and $\underline{\mathbf{I}}_{-c}^{f}$ are the restriction and prolongation operators, respectively, which are defined in the same way as for the nonlinear multigrid. Furthermore, the calls to the smoother refer to the application of a certain iterative method $\beta_{i}$ times. It is important to note that the system matrix on coarser levels can be obtained in two ways:

1. computing it with the coarse-grid discretization,

2. interpolating it using the projection operators,

$$
\underline{\mathbf{A}}^{c}=\underline{\mathbf{I}}_{f}^{c} \underline{\mathbf{A}}^{f} \underline{\mathbf{I}}_{c}^{f} .
$$

The second method is usually preferred as it is computationally cheaper.

For the coarse-grid problem, an initial guess, $\mathbf{E}^{c}=\mathbf{0}$ is made (Step 4 ). This is an adequate initial guess in linear multigrid methods since the coarse grid problem acts on the error and not directly on the solution [33]. 
At the last multigrid level, it is possible to solve the system completely (e.g. with a direct method) instead of further smoothing. This provides the advantage that the low frequency errors are completely eliminated on the lowest grid but makes the method computationally more intensive.

\subsubsection{Convergence of Linear Multigrid Methods}

If one chooses to pre- and post-smooth on the coarsest level, it is easy to describe the whole multigrid process as a matrix operation. Using the multigrid procedure described in Algorithm 6, after a V-cycle the error is reduced by

$$
\mathbf{E}_{n+1}=\underline{\mathbf{M}}_{M G} \mathbf{E}_{n} .
$$

For instance, the matrix $\underline{\mathbf{M}}_{M G}$ for a two-level multigrid that uses a splitting method as the smoother is [173]

$$
\underline{\mathbf{M}}_{M G}=\left(\underline{\mathbf{I}}-\underline{\mathbf{P}}^{-1} \underline{\mathbf{A}}\right)^{\beta_{1}} \underline{\mathbf{I}}_{c}^{f}\left(\underline{\mathbf{I}}-\underline{\mathbf{P}}_{c}^{-1} \underline{\mathbf{I}}_{f}^{c} \underline{\mathbf{A I}}_{c}^{f}\right)^{\beta_{1}+\beta_{2}} \underline{\mathbf{I}}_{f}^{c}\left(\underline{\mathbf{I}}-\underline{\mathbf{P}}^{-1} \underline{\mathbf{A}}\right)^{\beta_{2}}
$$

As a result, the rate of convergence of the linear multigrid method is determined by the eigenvalues of $\underline{\mathbf{M}}_{M G}$. Specifically, the multigrid method is stable only if

$$
\left|\rho\left(\underline{\mathbf{M}_{\mathbf{M G}}}\right)\right|<1
$$

where $\rho\left(\underline{\mathbf{M}}_{M G}\right)$ is the spectral radius of the matrix $\underline{\mathbf{M}}_{M G}$.

Equation (4.32) can be easily generalized to other multigrid cycles. However, given the size of the matrices and the number of matrix operations needed to compute $\underline{\mathbf{M}}_{M G}$, in practice the rate of convergence is estimated a posteriori from the computed norm of the residual. 



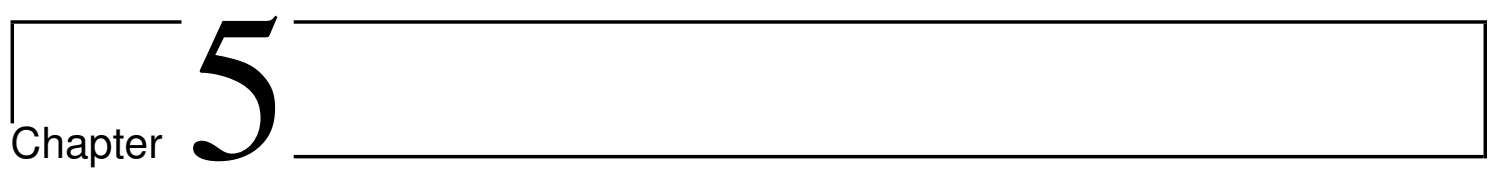

\section{Computing the Jacobian Matrix}

\section{Contents}

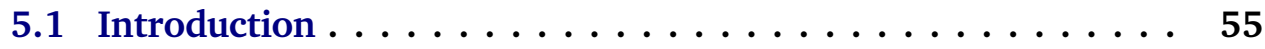

5.2 Numerical Jacobian and Optimizations . . . . . . . . . 57

5.2 .1 Coloring Technique . . . . . . . . . . . . . 58

5.2 .2 Accuracy . . . . . . . . . . . . . . . . . 59

5.3 Jacobian-Free Methods . . . . . . . . . . . . . . . . . . 60

5.4 Analytical Jacobian for DG Methods . . . . . . . . . . . . 61

5.4 .1 Advective Flux . . . . . . . . . . . . . . . . . . 61

5.4 .2 Advective Numerical Flux . . . . . . . . . . . . . . . . . 64

5.4 .3 Viscous Flux . . . . . . . . . . . . . . . . 65

5.4 .4 Viscous Numerical Fluxes . . . . . . . . . . . . . . . . . 69

5.5 Performance Comparison of the Jacobian Computation Methods 70

5.6 Final Remarks . . . . . . . . . . . . . . . . 71

\subsection{Introduction}

As was seen in Chapters 3 and 4, implicit time-integration methods imply the solution of several systems of linear equations of the form

$$
\underline{\mathbf{A}} \Delta \mathbf{Q}=\mathbf{B},
$$

where the Jacobian matrix, $\underline{A}$, depends on the solution at the previous step/iteration, $\mathbf{Q}_{0}$. In Section 4.3, several solution techniques for linear systems were reviewed. Direct, splitting and linear multigrid methods require the computation of the Jacobian matrix, whereas Krylov subspace methods only need the matrix action on a 
vector, $\underline{\mathbf{A X}}$. In this chapter, we specify methods to compute the Jacobian matrix, or at least to approximate the matrix action on a vector for a DG discretization.

The first and simplest strategy is the numerical computation of the DG Jacobian through a finite difference procedure, which is presented in Section 5.2. The main advantages of this method are that it is very simple to implement and that it provides good approximations to the Jacobian matrix. However, it is a very expensive way to obtain the matrix as it requires a number of evaluation of the spatial operators that is proportional to the number of degrees of freedom of the problem. For that reason, in Section 5.2.1 we review the coloring technique, a method based on graph theory that allows one to speed up the computation of the numerical DG Jacobian, still using the easy-to-implement finite difference method. The coloring method is a good alternative to the traditional numerical Jacobian since its coding complexity is not high and the performance of the method is significantly improved.

Although numerical Jacobians are easy to derive and code, they are very expensive to compute. Even using the coloring technique, the Jacobian computation times for practical problems become unaffordable.

An alternative to numerical Jacobians that is as simple to implement is the use of Jacobian- or matrix-free methods. They use the same finite difference derivation and can be used with any linear solver that only requires the matrix action, such as the Krylov subspace solvers of Section 4.3.3. Furthermore, since the Jacobian matrix is not constructed, these methods need much less storage. The derivation of matrix-free methods is explained in Section 5.3.

A third alternative is to compute the Jacobian analytically. This is a more complex task, but it provides a number of advantages over the methods listed before. First, the computation times are reduced significantly in comparison with the numerical Jacobian strategy. Second, the analytical Jacobian computation is less sensitive to round-off errors than the methods based on a finite difference. Third, having an assembled Jacobian matrix grants the possibility to use a larger variety of preconditioners and, as will be shown in Chapter 6, static-condensation methods. For these reasons, in this thesis we make use of the the analytical Jacobian. Section 5.4 provides a general derivation of the analytical Jacobian for general nonlinear advection-diffusion equations that is valid for all DG methods.

The amount of storage that is needed to save the Jacobian matrix in dense format is $\left(\mathrm{NDOF} \times n_{\text {cons }}\right)^{2} \times s_{\text {prec }}$, where $s_{\text {prec }}$ is the size of a variable with the desired precision. This quantity scales rapidly with the size of the problem and makes it impossible to use dense Jacobian matrices for practical problems. However, the Jacobian matrices for DG methods are block-sparse, i.e. most of the entries are zero and only some blocks of the matrices contain nonzero values. Furthermore, in multi-equation and multi-dimension problems, the nonzero blocks are very sparse themselves. Therefore, a variety of sparse formats are useful for storing the Jacobian, such as the Compressed Sparse Row (CSR) format, the Block Sparse Row (BSR) format, among others. Besides requiring less storage, these formats provide the advantage that fewer operations are needed for matrix operations, such 
as matrix-matrix and matrix-vector products.

\subsection{Numerical Jacobian and Optimizations}

In Section 4.2.1, it was shown that it is possible to solve the system of nonlinear equations generated by an implicit time integration scheme by solving several systems of linear equations that are obtained by expanding the nonlinear operator, $\mathbf{R}(\mathbf{Q})$, with a Taylor series,

$$
\mathbf{R}(\mathbf{Q})=\mathbf{R}\left(\mathbf{Q}_{0}\right)+\underline{\mathbf{A}\left(\mathbf{Q}_{0}\right)} \Delta \mathbf{Q}+\mathscr{O}\left((\Delta \mathbf{Q})^{2}\right)
$$

being

$$
\underline{\mathbf{A}}\left(\mathbf{Q}_{0}\right)=\left.\frac{\partial \mathbf{R}(\mathbf{Q})}{\partial \mathbf{Q}}\right|_{\mathbf{Q}_{0}}
$$

the Jacobian matrix.

This Taylor expansion can also be used to approximate the Jacobian matrix. For instance, one can assume that $\mathbf{Q}$ differs from $\mathbf{Q}_{0}$ only in one degree of freedom by a small quantity $\epsilon$,

$$
\mathbf{Q}=\mathbf{Q}_{0}+\epsilon \hat{\mathbf{K}}^{j},
$$

where $\hat{\mathbf{K}}$ is a vector whose entries are all zero, except in the $j$ position, where it is equal to one:

$$
\hat{k}_{i}^{j}= \begin{cases}1 & \text { if } i=j \\ 0 & \text { otherwise. }\end{cases}
$$

Equation (5.2) can then be reorganized as

$$
\underline{\mathbf{A}}^{j}=\frac{\mathbf{R}\left(\mathbf{Q}_{0}+\epsilon \hat{\mathbf{K}}^{j}\right)-\mathbf{R}\left(\mathbf{Q}_{0}\right)}{\epsilon}+\mathscr{O}\left(\epsilon^{2}\right) .
$$

If $\epsilon$ is small enough, the quantity $\underline{\mathbf{A}}^{j}$ is a good approximation of the $j^{\text {th }}$ column of the Jacobian matrix. Therefore, it is plain to see that the whole Jacobian matrix can be recovered by computing (5.6) NDOF times for $1 \leq j \leq \mathrm{NDOF} \times n_{\text {cons }}$, i.e. evaluating the nonlinear operator, $\mathbf{R}()$, NDOF +1 times. This approximation is first order accurate with respect to $\epsilon$. Higher-order finite difference approximations can be derived in a straightforward manner. For example, a second order accurate Jacobian computation is

$$
\underline{\mathbf{A}}^{j}=\frac{\mathbf{R}\left(\mathbf{Q}_{0}+\epsilon \hat{\mathbf{K}}^{j}\right)-\mathbf{R}\left(\mathbf{Q}_{0}-\epsilon \hat{\mathbf{K}}^{j}\right)}{2 \epsilon}+\mathscr{O}\left(\epsilon^{3}\right) .
$$

The drawback of (5.7) is that 2 NDOF evaluations of $\mathbf{R}()$ are needed.

Browne et al. [35] showed that equation 5.6 can yield second order accuracy if one uses a complex perturbation, $\epsilon \in \mathbb{C}$, with an extra advantage: smaller values of $\epsilon$ can be used without affecting the accuracy. 


\subsubsection{Coloring Technique}

If the degrees of freedom of a high-order DG discretization are numbered contiguously inside each element, the Jacobian matrix has a block structure. The block size is the number of degrees of freedom of each element. Moreover, the diagonal blocks of the Jacobian represent the contribution of the solution on the DOFs of an element to the solution on themselves, and the off-diagonal blocks represent the interaction between elements.

Each element is connected to the solution on neighbor elements if a compact DG discretization is used (such as in purely advective cases or in viscous cases with an IP discretization), whereas it is connected to the solution on neighbors of neighbor elements if a non-compact DG discretization is used (such as the BR1 viscous discretization). The difference between compact and non-compact schemes was discussed in Chapter 2 and is analyzed in depth in Section 5.4.

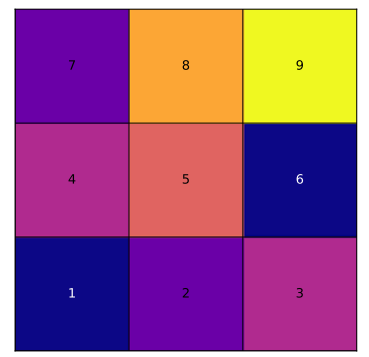

(a) $3 \times 3$ mesh and corresponding colors.

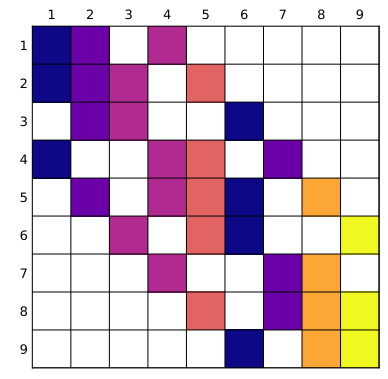

(b) Sparsity pattern for $3 \times 3$ mesh.

Figure 5.1: A simple 2D mesh of $3 \times 3$ and its corresponding sparsity pattern.

To illustrate the sparsity pattern of a high-order DG discretization, let us consider the small 2D mesh in Figure 5.1(a) (initially ignoring the colors). If we use a compact DG discretization, the sparsity pattern of the matrix (by blocks) corresponds to the one shown in Figure 5.1(b). Note that in each block column, $j_{\text {block}}$, the off-diagonal blocks that take nonzero values correspond to the elements that are connected to the element $j_{\text {block}}$. For instance, in the first block column, the only blocks that take nonzero values are the blocks one, two and four.

Since the numerical Jacobian method perturbs one DOF at a time to get the corresponding column of the Jacobian matrix, it is now evident that it is possible to perturb DOFs on elements that are far enough from each other (e.g. elements 1 and 6), so that the computed columns do not overlap. To take advantage, one can construct a graph that assigns a color to each element, so that elements with the same color do not share neighbors $[50,163]$ (or neighbors of neighbors, depending on the selected discretization). 
Once the domain is colored such that elements that do not affect each other's solutions have the same color (see for example Figure 5.1(a)), one can use (5.6) or (5.7) a reduced number of times to get all the entries of the Jacobian matrix. The vector $\mathbf{K}^{j}$ is replaced each time by another vector that takes the value of one in a specific DOF of different elements of the same color simultaneously.

As shown in Figure 5.1, in the small 2D mesh of 9 elements it is possible to get six colors. That means that by using the coloring technique, one can compute the Jacobian matrix numerically for the same cost as the traditional method would have for a mesh of six elements ${ }^{1}$. This may not seem a enormous improvement; however, if one considers larger meshes, the advantage is evident. For example, a mesh of $6 \times 6$ elements can be colored using only 7 colors (see Figure 5.2).

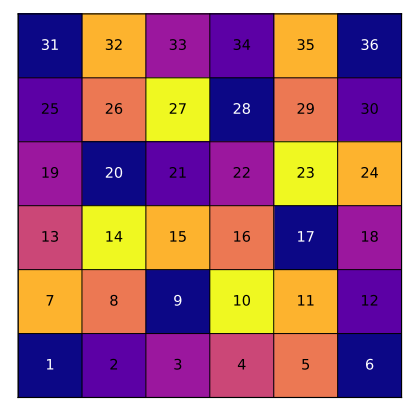

Figure 5.2: A simple 2D mesh of $6 \times 6$ and its corresponding sparsity pattern.

\subsubsection{Accuracy}

The accuracy of the finite difference numerical Jacobian depends strongly on the choice of $\epsilon$, as can be seen in Figure 5.3 for the first order approximation. If $\epsilon$ is too large, the finite difference is not able to capture the non-linearities of the problem appropriately; whereas if it is too small, round-off may contaminate the computation.

As a rule of thumb, $\epsilon$ must be something larger than the square root of the machine precision,

$$
\epsilon \gtrsim \sqrt{\epsilon_{m}}
$$

for the first order approximation, and

$$
\epsilon \gtrsim \sqrt[3]{\epsilon_{m} / 2}
$$

\footnotetext{
${ }^{1}$ This cost is relative to the number of evaluations of the nonlinear operator, R. However, the cost to evaluate the nonlinear spatial operator depends on the total number of elements of the mesh.
} 
for the second order approximation [3], where $\epsilon_{m} \approx 10^{-16}$ for 64-bit double precision. This approach assumes that $\mathbf{R}(\mathbf{Q})$ can be evaluated at machine precision. However, round-off errors tend to accumulate in the successive operations that are needed to compute $\mathbf{R}(\mathbf{Q})$. Therefore, several other approaches have been developed to select an appropriate parameter [108, 145].

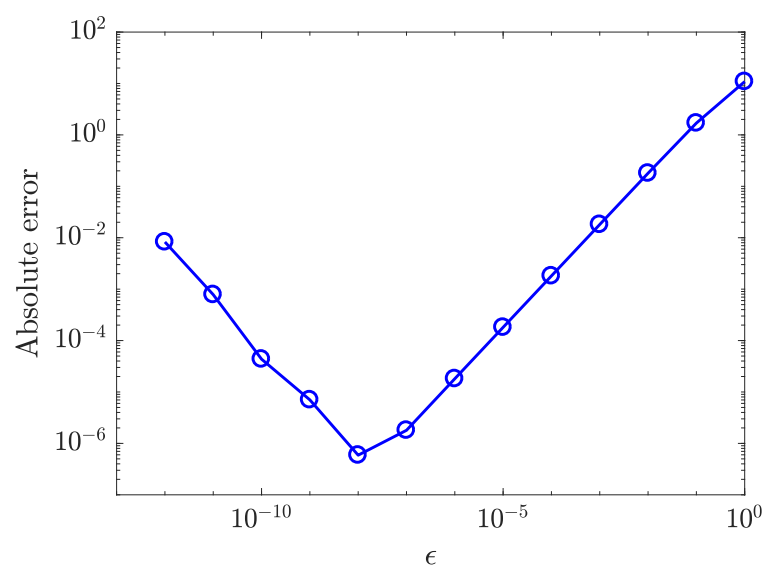

Figure 5.3: Absolute error of the numerical Jacobian as a function of $\epsilon$ (first order approximation with 64-bit double precision variables).

\subsection{Jacobian-Free Methods}

Matrix- or Jacobian-Free methods are a class of methods that can be used to get the solution of the linear system without having to assemble the Jacobian matrix $[82,108]$. They use the same finite difference strategy of numerical Jacobians and are applicable to iterative methods that only need matrix vector products in the solver process, such as some classic splitting methods (Section 4.3.2) and Krylov subspace methods (Section 4.3.3). The product $\underline{\mathrm{AV}}$ can be easily approximated using (5.6) as

$$
\underline{\mathbf{A V}} \approx \frac{\mathbf{R}\left(\mathbf{Q}_{0}+\epsilon \mathbf{V}\right)-\mathbf{R}\left(\mathbf{Q}_{0}\right)}{\epsilon},
$$

for first order accuracy, or (5.6) as

$$
\underline{\mathbf{A V}} \approx \frac{\mathbf{R}\left(\mathbf{Q}_{0}+\epsilon \mathbf{V}\right)-\mathbf{R}\left(\mathbf{Q}_{0}-\epsilon \mathbf{V}\right)}{2 \epsilon}
$$

for second order accuracy.

Jacobian-free methods can be very efficient. However, their main disadvantages are that they are sensitive to round-off errors and that it is not straightforward to obtain a good preconditioner. A workaround for the latter is to compute the 
Jacobian matrix from time to time and use it for preconditioning during several iterations. In any case, the matrix-free $\mathbf{A V}$ operation contains all the information

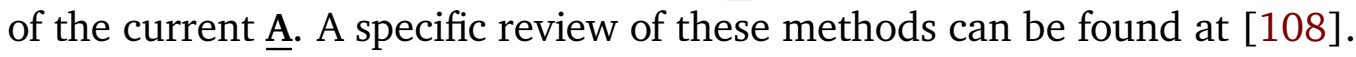

\subsection{Analytical Jacobian for DG Methods}

There are inevitably some cases where the Jacobian matrix is needed, either for preconditioning, for stability analysis or for linear solvers that need the full Jacobian matrix. The most efficient way to assemble the Jacobian is to compute it analytically. For that reason, in this section we derive the analytical expressions for obtaining the Jacobian matrix of a standard DG discretization of a conservation law with nonlinear advective and diffusive terms,

$$
\partial_{t} \mathbf{q}+\vec{\nabla} \cdot\left(\overleftrightarrow{\mathbf{f}}^{a}-\overleftrightarrow{\mathbf{f}}^{v}\right)=\mathbf{0}
$$

The strategy used here starts from the DG discretized equation and linearizes locally by means of a Taylor expansion. The DG discretization (2.7) can be rewritten as

$$
\int_{\Omega} \partial_{t} \mathbf{q}^{N} \phi_{j} \mathrm{~d} \Omega-\int_{\Omega}\left(\stackrel{\mathrm{f}}{ }^{a N}-\overleftrightarrow{\mathbf{f}}^{v N}\right) \cdot \vec{\nabla} \phi_{j} \mathrm{~d} \Omega+\int_{\partial \Omega}\left(\hat{\mathbf{f}}^{a}-\hat{\mathbf{f}}^{v}\right) \phi_{j} \mathrm{~d} S=\mathbf{0} .
$$

A few remarks can be made. First, the flux is explicitly written as its advective and diffusive parts. Furthermore, the source term is omitted since its influence is restricted to the right-hand-side and it is not important for the derivations in next sections. Finally, the superscript $e$ is dropped for readability. However, the reader should keep in mind that this equation, and therefore the derivations henceforth (in Sections 5.4.1 - 5.4.4), represents the nonlinear system that corresponds to the degree of freedom $j$ of a specific element $e$.

\subsubsection{Advective Flux}

To facilitate the derivation, let us first consider the purely advective equations (e.g. the compressible Euler equations of gas dynamics),

$$
\int_{\Omega} \partial_{t} \mathbf{q}^{N} \phi_{j} \mathrm{~d} \Omega-\int_{\Omega} \overleftrightarrow{\mathbf{f}}^{a}\left(\mathbf{q}^{N}\right) \cdot \vec{\nabla} \phi_{j} \mathrm{~d} \Omega+\int_{\partial \Omega} \hat{\mathbf{f}}^{a}\left(\mathbf{q}^{+}, \mathbf{q}^{-}, \vec{n}\right) \phi_{j} \mathrm{~d} S=\mathbf{0},
$$

where the interpolant of the flux, $\stackrel{\mathfrak{f}}{a N}$, is nothing but the flux evaluated with the discrete solution, $\stackrel{\mathbf{f}}{ }^{a}\left(\mathbf{q}^{N}\right)$, and $\mathbf{q}^{+}$and $\mathbf{q}^{-}$represent the discrete solution on both sides of the interface $\partial \Omega$. 
The first step is to linearize the advective flux using a Taylor expansion:

$$
\begin{aligned}
\overleftrightarrow{\mathbf{f}}^{a}(\mathbf{q}) & =\overleftrightarrow{\mathbf{f}}^{a}\left(\mathbf{q}_{0}\right)+\frac{\partial \overleftrightarrow{\mathbf{f}}^{a}}{\partial \mathbf{q}} \Delta \mathbf{q}+\mathscr{O}\left((\Delta \mathbf{q})^{2}\right) \\
& \approx \overleftrightarrow{\mathbf{f}}^{a}\left(\mathbf{q}_{0}\right)+\underline{\mathbf{J}}^{a} \Delta \mathbf{q},
\end{aligned}
$$

where $\underline{\mathbf{J}}^{a}$ is the Jacobian of the advective flux evaluated with $\mathbf{q}_{0}$. The Jacobians for the advective terms of the Euler/Navier-Stokes equations can be found in Appendix A.1.1.

A linearized expression for the advective numerical flux of an internal interface can be obtained in the same way, now taking into account that it depends on the solution on both sides of the interface:

$$
\begin{aligned}
\left.\hat{\mathbf{f}}^{a}\left(\mathbf{q}^{+}, \mathbf{q}^{-}, \vec{n}\right)\right|_{\partial \Omega \backslash \Gamma} & =\hat{\mathbf{f}}^{a}\left(\mathbf{q}_{0}^{+}, \mathbf{q}_{0}^{-}, \vec{n}\right)+\frac{\partial \hat{\mathbf{f}}^{a}}{\partial \mathbf{q}^{+}} \Delta \mathbf{q}^{+}+\frac{\partial \hat{\mathbf{f}}^{a}}{\partial \mathbf{q}^{-}} \Delta \mathbf{q}^{-}+\mathscr{O}\left(\max \left(\left(\Delta \mathbf{q}^{+}\right)^{2},\left(\Delta \mathbf{q}^{-}\right)^{2}\right)\right) \\
& \approx \hat{\mathbf{f}}^{a}\left(\mathbf{q}_{0}^{+}, \mathbf{q}_{0}^{-}, \vec{n}\right)+\hat{\mathbf{f}}_{\mathbf{q}^{a}}^{a} \Delta \mathbf{q}^{+}+\hat{\mathbf{f}}_{\mathbf{q}^{-}}^{a} \Delta \mathbf{q}^{-} .
\end{aligned}
$$

In (5.17), $\hat{\mathbf{f}}_{\mathbf{q}^{ \pm}}^{a}$ denote the Jacobians of the advective numerical flux function with respect to $\mathbf{q}^{+}$and $\mathbf{q}^{-}$evaluated in $\mathbf{q}_{0}^{ \pm}$. Inserting the linearized fluxes, (5.15) and (5.17), into (5.14) yields

$$
\begin{aligned}
\int_{\Omega} \partial_{t} \mathbf{q}^{N} \phi_{j} \mathrm{~d} \Omega-\int_{\Omega} \underline{\mathbf{J}}^{a} \Delta \mathbf{q}^{N} \cdot \vec{\nabla} \phi_{j} \mathrm{~d} \Omega+\int_{\partial \Omega}\left(\hat{\mathbf{f}}_{\mathbf{q}^{+}}^{a} \Delta \mathbf{q}^{+}+\hat{\mathbf{f}}_{\mathbf{q}^{a}}^{a} \Delta \mathbf{q}^{-}\right) \phi_{j} \mathrm{~d} S= \\
\int_{\Omega} \overleftrightarrow{\mathbf{f}}^{N}\left(\mathbf{q}_{0}^{N}\right) \cdot \vec{\nabla} \phi_{j} \mathrm{~d} \Omega-\int_{\partial \Omega} \hat{\mathbf{f}}^{a}\left(\mathbf{q}_{0}^{+}, \mathbf{q}_{0}^{-}, \vec{n}\right) \phi_{j} \mathrm{~d} S .
\end{aligned}
$$

It is important to note that when the face of an element belongs to a physical domain boundary, $\partial \Omega \subseteq \Gamma$, the solution on the outer side of the face may depend on the solution on the inner side because of the boundary condition, $\mathbf{q}^{-}\left(\mathbf{q}^{+}\right)$. Therefore, the numerical flux function only depends on the solution on the inner side of the face, $\hat{\mathbf{f}}^{a}\left(\mathbf{q}^{+}, \vec{n}\right)$. As a consequence, (5.17) on a domain boundary is actually

$$
\left.\hat{\mathbf{f}}^{a}\left(\mathbf{q}^{+}, \vec{n}\right)\right|_{\partial \Omega \Omega \Gamma} \approx \hat{\mathbf{f}}^{a}\left(\mathbf{q}_{0}^{+}, \vec{n}\right)+\left(\hat{\mathbf{f}}_{\mathbf{q}^{+}}^{a}+\hat{\mathbf{f}}_{\mathbf{q}^{-}}^{a} \mathbf{q}_{\mathbf{q}^{+}}^{-}\right) \Delta \mathbf{q}^{+},
$$

where $\mathbf{q}_{\mathbf{q}^{+}}^{-}=\partial \mathbf{q}^{-} / \partial \mathbf{q}^{+}$is the Jacobian of the "Dirichlet" boundary condition.

Moreover, one can also replace $\mathbf{q}^{N}$ and $\overleftrightarrow{\mathbf{f}}^{N}$ by polynomial expansions, as they were defined in (2.5),

$$
\begin{aligned}
\mathbf{q}^{N} & =\sum_{r=1}^{\mathrm{NDOF}^{e}} \mathbf{q}_{r}^{N} \phi_{r}(\vec{x}) \leftarrow \mathbf{q}_{r}^{N} \phi_{r}, \\
\stackrel{\mathbf{f}}{N}^{N} & =\sum_{r=1}^{\mathrm{NDOF}^{e}} \overleftrightarrow{\mathbf{f}}_{r}^{N} \phi_{r}(\vec{x}) \leftarrow \stackrel{\mathbf{f}}{r}_{r}^{N} \phi_{r} .
\end{aligned}
$$


Note that we make use of Einstein notation convention (Section 1.5) to simplify the mathematical expressions. Equation (5.18) then becomes

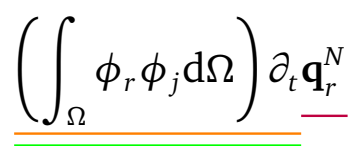

$$
\begin{aligned}
& +\left(-\int_{\Omega}\left(\underline{\mathbf{J}}^{a} \phi\right)_{r} \cdot \vec{\nabla} \phi_{j} \mathrm{~d} \Omega+\int_{\partial \Omega} \hat{\mathbf{f}}_{\mathbf{q}^{+}}^{a} \phi_{r} \phi_{j} \mathrm{~d} S+\int_{\partial \Omega \cap \Gamma} \hat{\mathbf{f}}_{\mathbf{q}^{-}}^{a} \mathbf{q}_{\mathbf{q}^{+}}^{-} \phi_{r} \phi_{j} \mathrm{~d} S\right) \Delta \mathbf{q}_{r}^{N} \\
& +\left(\int_{\partial \Omega \backslash \Gamma} \hat{\mathbf{f}}_{\mathbf{q}^{-}}^{a} \phi_{r}^{-} \phi_{j} \mathrm{~d} S\right) \Delta \mathbf{q}_{r}^{N}=\underline{\hat{\mathbf{b}}_{j}^{a}}
\end{aligned}
$$

where $\phi_{r}^{-}$denotes the shape function corresponding to the degree of freedom $r$ of an external element $(\neq e), \mathbf{q}_{r}^{N}$ is the solution state vector on that external degree of freedom $r, \hat{\mathbf{b}}_{j}^{a}$ is the advective contribution to the right-hand-side that only depends on known values of the solution $\left(\mathbf{q}_{0}^{N}\right)$.

The block structure of the Jacobian matrix can be inferred from (5.22) and is illustrated in Figure 5.4 for the simple 2D mesh of Figure 5.1(a), where the diagonal blocks are marked in blue and the off-diagonal blocks in cyan. If we suppose that we are analyzing the first degree of freedom, $j=1$, of element $e=3$, (5.22) can be interpreted as the contribution to the red and orange blocks of the Jacobian matrix, and to the green array of the RHS (these colors match with the underlining of the equation). The first term contributes to both the orange block and the RHS in amounts that depend on the discretization of the time derivatives (not treated here). The second term is the advective contribution to the orange block, and includes the action of the top and left boundaries. The third term is the advective contribution to the off-diagonal blocks two and six (in red), since those are the only elements that own shape functions that take nonzero values on any faces of element $e=3\left(\partial \Omega^{3} \backslash \Gamma\right)$. Finally, $\hat{\mathbf{b}}_{j}^{a}$ is the spatial contribution to the RHS of element $e$ for the degree of freedom $j$. Note that the surface integrals on physical boundaries $(\Gamma)$ only contribute to the diagonal blocks of the Jacobian matrix and that, in the advective case, the volume integrals contribute only to the diagonal blocks of the Jacobian matrix.

In a nutshell, (5.22) can be rewritten in matrix form for all the DOFs of the element as

$$
\underline{\mathbf{M}} \partial_{t} \mathbf{Q}^{N}+\underline{\hat{\mathbf{A}}}^{a} \Delta \mathbf{Q}^{N}+\underline{\hat{A}}^{a} \Delta \mathbf{Q}^{N}=\hat{\mathbf{B}}^{a},
$$

where $\underline{\mathbf{M}}$ is the elemental mass matrix, $\underline{\hat{\mathbf{A}}}^{a}$ is the advective contribution to the diagonal block of element $e$, and $\underline{\hat{A}}^{a}$ is the advective contribution to the off-diagonal blocks of element $e$. Multiplying on the left by the inverse of the mass matrix and inserting a specific time-integration method we get

$$
\left(\underline{\mathbf{A}}^{T}+\underline{\mathbf{A}}^{a}\right) \Delta \mathbf{Q}^{N}+\underline{\mathbf{A}}^{\underline{a}} \Delta \mathbf{Q}^{\underline{N}}=\mathbf{B}^{a \cup T},
$$




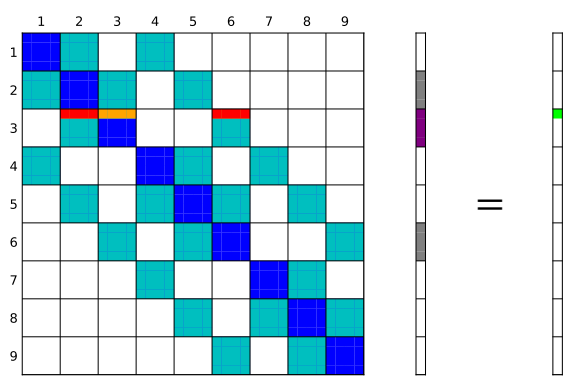

Figure 5.4: Linear system, $\mathbf{A} \Delta \mathbf{Q}=\mathbf{B}$, that results from the time-implicit DG discretization on the simple 2D mesh of Figure 5.1(a). The contribution of a specific degree of freesom is highlighted.

where $\underline{A}^{T}=\partial\left(\partial_{t} \mathbf{Q}^{N}\right) / \partial \mathbf{Q}^{N}$ is the temporal contribution to the diagonal block of element $e, \underline{A}^{a}$ is the advective contribution to the diagonal block of element $e$ (now including the mass matrix terms), $\underline{A}^{a}$ is the advective contribution to the off-diagonal blocks of element $e$ including the mass matrix terms, and $\mathbf{B}^{a \cup T}$ is the total RHS (with temporal and advective contributions) of element $e$ after taking into account the mass matrix action.

\subsubsection{Advective Numerical Flux}

The Jacobian of the advective numerical flux, $\hat{\mathbf{f}}_{\mathbf{q}^{ \pm}}^{a}$, is needed to assemble the analytical Jacobian. It can be obtained numerically with a finite difference, as explained in Section 5.2, or analytically. The former alternative may seem inadequate, given that the intention is to reduce the computational cost of computing the Jacobian matrix. However, $\hat{\mathbf{f}}_{\mathbf{q}^{ \pm}}^{a}$ needs only to be computed on the faces and it is only an $n_{\text {cons }} \times n_{\text {cons }}$ matrix; therefore, it is inexpensive to compute compared to the cost of computing the whole Jacobian matrix.

The optimal choice is to compute $\hat{\mathbf{f}}_{\mathbf{q}^{ \pm}}^{a}$ analytically. Given the large variety of advective numerical fluxes available in the literature [193], we only show here the derivation of the Jacobian for the Lax-Friedrichs numerical flux.

From (2.19), the Lax-Friedrichs numerical flux is

$$
\hat{\mathbf{f}}_{L F}^{a}\left(\mathbf{q}^{+}, \mathbf{q}^{-}, \vec{n}\right)=\frac{1}{2}\left(\overleftrightarrow{\mathbf{f}}^{a}\left(\mathbf{q}^{+}\right) \cdot \vec{n}+\stackrel{\leftrightarrow}{\mathbf{f}}^{a}\left(\mathbf{q}^{-}\right) \cdot \vec{n}+\lambda\left(\mathbf{q}^{+}-\mathbf{q}^{-}\right)\right)
$$

where $\lambda=\max \left(\tilde{\lambda}\left(\mathbf{q}^{+}\right), \tilde{\lambda}\left(\mathbf{q}^{-}\right)\right)$is the largest eigenvalue of the linearized advective flux over $\mathbf{q}^{+}$and $\mathbf{q}^{-}$.

Therefore, the derivative of the Lax-Friedrichs with respect to the state vector 
of the solution on both sides of the interface yields [91]

$$
\frac{\partial \hat{\mathbf{f}}_{L F}^{a}}{\partial \mathbf{q}^{ \pm}}=\hat{\mathbf{f}}_{\mathbf{q}^{ \pm}}^{a}=\frac{1}{2}\left({\stackrel{\mathbf{f}}{\mathbf{q}^{ \pm}}}^{ \pm} \cdot \vec{n} \pm \underline{\mathbf{I}} \lambda+\left(\mathbf{q}^{+}-\mathbf{q}^{-}\right) \otimes\left[\frac{\partial \lambda}{\partial \mathbf{q}^{ \pm}}\right]\right),
$$

where $\partial \lambda / \partial \mathbf{q} \pm$ depends on the physics. For the compressible Euler/Navier-Stokes equations they are

$$
\begin{aligned}
& \frac{\partial \lambda}{\partial \mathbf{q}^{+}}= \begin{cases}\operatorname{sign}\left(v_{n}\left(\mathbf{q}^{+}\right)\right) v_{n}^{\prime}\left(\mathbf{q}^{+}\right)+c^{\prime}\left(\mathbf{q}^{+}\right) & \text {if } \tilde{\lambda}\left(\mathbf{q}^{+}\right) \geq \tilde{\lambda}\left(\mathbf{q}^{-}\right), \\
\mathbf{0} & \text { otherwise, }\end{cases} \\
& \frac{\partial \lambda}{\partial \mathbf{q}^{-}}= \begin{cases}\operatorname{sign}\left(v_{n}\left(\mathbf{q}^{-}\right)\right) v_{n}^{\prime}\left(\mathbf{q}^{-}\right)+c^{\prime}\left(\mathbf{q}^{-}\right) & \text {if } \tilde{\lambda}\left(\mathbf{q}^{+}\right)<\tilde{\lambda}\left(\mathbf{q}^{-}\right), \\
\mathbf{0} & \text { otherwise. }\end{cases}
\end{aligned}
$$

The derivatives for the normal velocity and the speed of sound are

$$
\begin{aligned}
v_{n}^{\prime} & =\left[\begin{array}{lllll}
-v_{n} / \rho & n_{1} / \rho & n_{2} / \rho & n_{3} / \rho & 0
\end{array}\right]^{T}, \\
c^{\prime} & =\left[\begin{array}{lllll}
\left(\|\mathbf{v}\|_{2}-E\right) / \rho & -u / \rho & -v / \rho & -w / \rho & 1 / \rho
\end{array}\right]^{T} \frac{\gamma(\gamma-1)}{2 c} .
\end{aligned}
$$

\subsubsection{Viscous Flux}

Whether we use the conservative gradients approach (Section 2.1.1), the primitive gradients approach, or the whole flux approach (Appendix B), we get different mathematical expressions for the Jacobian of the viscous terms. In this section, we derive the DG analytical Jacobian for the conservative gradients approach, since that is the viscous discretization used throughout this thesis and in HORSES3D.

We consider (5.13) without the time derivative and without the advective fluxes to facilitate the analysis. Rewriting (5.13) with the explicit dependencies yields

$$
\int_{\Omega} \overleftrightarrow{\mathbf{f}}^{v}\left(\mathbf{q}^{N}, \overleftrightarrow{\mathbf{g}}^{N}\right) \cdot \vec{\nabla} \phi_{j} \mathrm{~d} \Omega-\int_{\partial \Omega} \hat{\mathbf{f}}^{v}\left(\mathbf{q}^{+}, \vec{\nabla} \mathbf{q}^{+}, \mathbf{q}^{-}, \vec{\nabla} \mathbf{q}^{-}, \vec{n}\right) \phi_{j} \mathrm{~d} \Omega=\mathbf{0},
$$

for a compact scheme, or

$$
\int_{\Omega} \overleftrightarrow{\mathbf{f}}^{v}\left(\mathbf{q}^{N}, \overleftrightarrow{\mathbf{g}}^{N}\right) \cdot \vec{\nabla} \phi_{j} \mathrm{~d} \Omega-\int_{\partial \Omega} \hat{\mathbf{f}}^{v}\left(\mathbf{q}^{+}, \overleftrightarrow{\mathbf{g}}^{+}, \mathbf{q}^{-}, \stackrel{\mathrm{g}}{ }^{-}, \vec{n}\right) \phi_{j} \mathrm{~d} \Omega=\mathbf{0},
$$

for a non-compact scheme (see Section 2.1.3). As was explained in Section 2.1.3, in the non-compact scheme, the viscous numerical flux depends on the DG gradients of neighbor elements, which in turn depend on the solution of their neighbors. That causes a neighbors of neighbors coupling which affects the sparsity pattern of the Jacobian matrix. Figure 5.5 illustrates this phenomenon for the nine-element example considered here. 


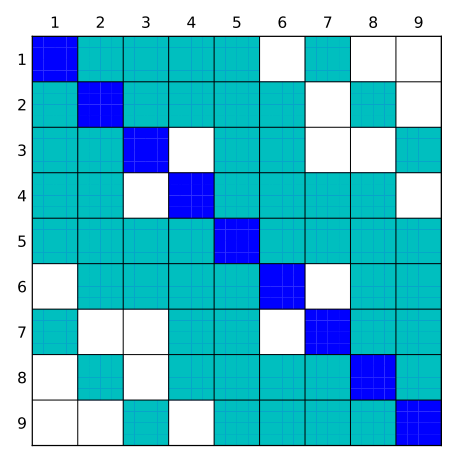

Figure 5.5: Sparsity pattern for the mesh of Figure 5.1(a) when using a noncompact viscous numerical flux.

A denser Jacobian matrix means that more storage is needed (even using sparse matrix formats), that more operations are needed to assemble the matrix, and usually that the linear solver will take longer to solve the resulting linear system. In fact, the formulation of the analytical Jacobian and its computational implementation are much more difficult, especially when using distributed memory parallelization (MPI), since the information and connectivities of the first two layers of elements on an MPI interface must be passed between partitions. Therefore, in what remains of this chapter, we only consider compact viscous numerical fluxes to obtain Jacobian matrices with sparsity patterns as in Figure 5.4.

We start by obtaining linearized versions of the viscous flux, which now depends on $\mathbf{q}$ and $\overleftrightarrow{\mathbf{g}}$,

$$
\begin{aligned}
\overleftrightarrow{\mathbf{f}}^{v}(\mathbf{q}, \overleftrightarrow{\mathbf{g}}) & =\overleftrightarrow{\mathbf{f}}^{v}\left(\mathbf{q}_{0}, \overleftrightarrow{\mathbf{g}}_{0}\right)+\frac{\partial \overleftrightarrow{\mathbf{f}}^{v}}{\partial \mathbf{q}} \Delta \mathbf{q}+\frac{\partial \overleftrightarrow{\mathbf{f}}^{v}}{\partial \overleftrightarrow{\mathbf{g}}} \Delta \overleftrightarrow{\mathbf{g}}+\mathscr{O}\left(\max \left((\Delta \mathbf{q})^{2},(\Delta \overleftrightarrow{\mathbf{g}})^{2}\right)\right) \\
& \approx \overleftrightarrow{\mathbf{f}}^{v}\left(\mathbf{q}_{0}, \overleftrightarrow{\mathbf{g}}_{0}\right)+\underline{\mathbf{J}}^{v} \Delta \mathbf{q}+\underline{\underline{\mathbf{G}}} \Delta \overleftrightarrow{\mathbf{g}}
\end{aligned}
$$

and of the viscous numerical flux,

$$
\begin{aligned}
\hat{\mathbf{f}}^{v}\left(\mathbf{q}^{+}, \vec{\nabla} \mathbf{q}^{+}, \mathbf{q}^{-}, \vec{\nabla} \mathbf{q}^{-}, \vec{n}\right)= & \hat{\mathbf{f}}^{v}\left(\mathbf{q}_{0}^{+}, \vec{\nabla} \mathbf{q}_{0}^{+}, \mathbf{q}_{0}^{-}, \vec{\nabla} \mathbf{q}_{0}^{-}, \vec{n}\right) \\
& +\frac{\partial \hat{\mathbf{f}}^{v}}{\partial \mathbf{q}^{+}} \Delta \mathbf{q}^{+}+\frac{\partial \overrightarrow{\mathbf{f}^{v}}}{\partial \vec{\nabla} \mathbf{q}^{+}} \Delta\left(\vec{\nabla} \mathbf{q}^{+}\right) \\
& +\frac{\partial \hat{\mathbf{f}}^{v}}{\partial \mathbf{q}^{-}} \Delta \mathbf{q}^{-}+\frac{\partial \overrightarrow{\mathbf{f}^{v}}}{\partial \vec{\nabla} \mathbf{q}^{-}} \Delta\left(\vec{\nabla} \mathbf{q}^{-}\right)+\mathscr{O}\left(\max \left(\left(\Delta \mathbf{q}^{+}\right)^{2},\left(\Delta \mathbf{q}^{-}\right)^{2}\right)\right) \\
\approx \hat{\mathbf{f}}_{0}^{v} & +\hat{\mathbf{f}}_{\mathbf{q}^{+}}^{v} \Delta \mathbf{q}^{+}+\hat{\mathbf{f}}_{\vec{\nabla} \mathbf{q}^{+}}^{v} \Delta\left(\vec{\nabla} \mathbf{q}^{+}\right)+\hat{\mathbf{f}}_{\mathbf{q}^{-}}^{v} \Delta \mathbf{q}^{-}+\hat{\mathbf{f}}_{\vec{\nabla} \mathbf{q}^{-}}^{v} \Delta\left(\vec{\nabla} \mathbf{q}^{-}\right) .(5.34)
\end{aligned}
$$

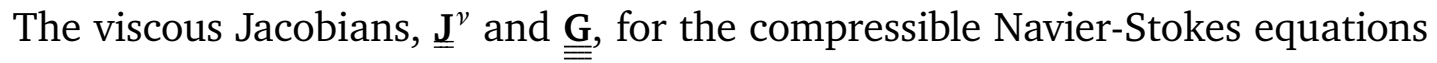
can be found in Appendix A.1.1, and the Jacobians of the viscous numerical flux 
are analyzed in Section 5.4.4. Again, all Jacobians must be evaluated at the initial state, $\mathbf{q}_{0}^{N}, \overleftrightarrow{\mathbf{g}}{ }_{0}^{N}, \mathbf{q}_{0}^{ \pm}, \vec{\nabla} \mathbf{q}_{0}^{ \pm}$.

Following the same procedure as in previous section, we take (5.31), replace the viscous flux and the viscous numerical flux by their linearized versions, (5.33) and (5.34), insert the definitions of the polynomial discretization of $\mathbf{q}^{N}$ and $\overleftrightarrow{\mathbf{f}}^{N}$ from (5.20), and define the additional polynomial expansions,

$$
\begin{aligned}
\overleftrightarrow{\mathbf{g}}^{N} & =\sum_{m=1}^{\mathrm{NDOF}^{e}} \overleftrightarrow{\mathbf{g}}_{m} \phi_{m}(\vec{x}) \leftarrow \overleftrightarrow{\mathbf{g}}_{m} \phi_{m}, \\
\vec{\nabla} \mathbf{q}^{N} & =\sum_{r=1}^{\mathrm{NDOF}^{e}} \mathbf{q}_{r} \vec{\nabla} \phi_{r}(\vec{x}) \leftarrow \mathbf{q}_{r} \vec{\nabla} \phi_{r},
\end{aligned}
$$

to obtain a general expression for the viscous terms:

$$
\begin{aligned}
& \underline{\left(\int_{\Omega}\left(\underline{\mathbf{J}}^{v} \phi\right)_{r} \cdot \vec{\nabla} \phi_{j} \mathrm{~d} \Omega\right) \underline{\Delta \mathbf{q}_{r}^{N}}+\left(\int_{\Omega}(\underline{\underline{\underline{G}}} \phi)_{m} \cdot \vec{\nabla} \phi_{j} \mathrm{~d} \Omega\right) \cdot \underline{\underline{\Delta \overleftrightarrow{\mathbf{g}}_{m}^{N}}}} \\
& -\left(\int_{\partial \Omega \backslash \Gamma}\left(\hat{\mathbf{f}}_{\mathbf{q}^{+}}^{v} \phi_{r}+\hat{\mathbf{f}}_{\vec{\nabla} \mathbf{q}^{+}}^{v} \cdot \vec{\nabla} \phi_{r}\right) \phi_{j} \mathrm{~d} \Omega\right) \Delta \mathbf{q}_{r}^{N} \\
& -\left(\int_{\partial \Omega \cap \Gamma}\left(\frac{\partial \hat{\mathbf{f}}_{\Gamma}^{v}}{\partial \mathbf{q}^{+}} \phi_{r}+\frac{\partial \hat{\mathbf{f}}_{\Gamma}^{v}}{\partial \vec{\nabla} \mathbf{q}^{+}} \cdot \vec{\nabla} \phi_{r}\right) \phi_{j} \mathrm{~d} \Omega\right) \Delta \mathbf{q}_{r}^{N} \\
& -\left(\int_{\partial \Omega \backslash \Gamma}\left(\hat{\mathbf{f}}_{\mathbf{q}^{-}}^{v} \phi_{r}^{-}+\hat{\mathbf{f}}_{\vec{\nabla} \mathbf{q}^{-}}^{v} \vec{\nabla} \phi_{r}^{-}\right) \phi_{j} \mathrm{~d} \Omega\right) \Delta \mathbf{q}_{r}^{\frac{N}{2}}=\hat{\mathbf{b}}_{j}^{v} .
\end{aligned}
$$

In this work, (5.37) is called the outer equation since the quantity $\overleftrightarrow{\mathbf{g}}_{m}^{N}$ must be obtained from the DG discretization of the gradients, i.e. the inner equation.

Several remarks can be made:

- Note that the index for the Einstein summation convention of the second volume integral was changed, $r \leftarrow m$. This change was deliberate since it will facilitate the insertion of the inner equation.

- For some of the terms of (5.37), it is now evident where their contributions are located in the linear system of Figure 5.4, as they are signaled with the colored underlines for the example $e=3, j=1$. At this point, it is not evident to which part of the system the second volume integral contributes. However, it can be deduced that it contributes to both the diagonal block (in orange) and the off-diagonal block (in red) from the analysis in Section 5.4.3.1.

- The Jacobian of the numerical fluxes on the faces that belong to the physical boundaries, $\partial \Omega \subseteq \Gamma$, can be obtained numerically using a finite difference 
method, or analytically, in which case they can be expressed as

$$
\begin{aligned}
\frac{\partial \hat{\mathbf{f}}_{\Gamma}^{v}}{\partial \mathbf{q}^{+}} & =\hat{\mathbf{f}}_{\mathbf{q}^{+}}^{v}+\hat{\mathbf{f}}_{\mathbf{q}^{-}}^{v} \mathbf{q}_{\mathbf{q}^{+}}^{-}+\hat{\mathbf{f}}_{\vec{\nabla} \mathbf{q}^{-}}^{v}\left(\vec{\nabla} \mathbf{q}^{-}\right)_{\mathbf{q}^{+}}, \text {and } \\
\frac{\partial \hat{\mathbf{f}}_{\Gamma}^{v}}{\partial \vec{\nabla} \mathbf{q}^{+}} & =\hat{\mathbf{f}}_{\vec{\nabla} \mathbf{q}^{+}}^{v}+\hat{\mathbf{f}}_{\vec{\nabla} \mathbf{q}^{-}}^{v}\left(\vec{\nabla} \mathbf{q}^{-}\right)_{\vec{\nabla} \mathbf{q}^{+}},
\end{aligned}
$$

where $\mathbf{q}_{\mathbf{q}^{+}}^{-}$is again the Jacobian of the Dirichlet boundary condition, and $\left(\vec{\nabla} \mathbf{q}^{-}\right)_{\mathbf{q}^{+}}$and $\left(\vec{\nabla} \mathbf{q}^{-}\right)_{\vec{\nabla} \mathbf{q}^{+}}$are the Jacobians of the Neumann boundary condition.

- Equation (5.37) can be rewritten in matrix form for all the degrees of freedom of a specific element as

$$
\underline{\mathbf{A}}^{v_{1}} \Delta \mathbf{Q}^{N}+\underline{\mathbf{A}}^{v_{2}} \cdot \Delta \stackrel{\leftrightarrow}{\mathbf{G}}^{N}+\underline{\mathbf{A}}^{v_{1}} \Delta \mathbf{Q}^{N}=\mathbf{B}^{v}
$$

\subsubsection{Inner Equation}

The variable $\overleftrightarrow{\mathbf{g}}^{N}$ is the DG-discretized gradient, $(\vec{\nabla} \mathbf{q})^{N}$, which was obtained in (2.17) as

$$
\int_{\Omega} \overleftrightarrow{\mathbf{g}}^{N} \phi_{m} \mathrm{~d} \Omega=-\int_{\Omega} \mathbf{q}^{N} \vec{\nabla} \phi_{m} \mathrm{~d} \Omega+\int_{\partial \Omega} \hat{\mathbf{q}} \phi_{m} \vec{n} \mathrm{~d} S .
$$

Note that we changed the index of the analyzed basis function, $j \leftarrow m$, to couple this equation with (5.37).

Following the procedure of previous sections, (5.41) is linearized around $\mathbf{q}_{0}^{N}$ and the unknown terms, $\mathbf{q}^{N}$, are replaced by their discrete definition (a polynomial expansion) to obtain

$$
\begin{aligned}
\left(\int_{\Omega} \phi_{r} \phi_{m} \mathrm{~d} \Omega\right) \overleftrightarrow{\mathbf{g}}_{r}^{N}= & \\
\left(-\int_{\Omega} \phi_{r} \vec{\nabla} \phi_{m} \mathrm{~d} \Omega\right. & \left.+\int_{\partial \Omega} \hat{\mathbf{q}}_{\mathbf{q}^{+}} \phi_{r} \phi_{m} \vec{n} \mathrm{~d} S+\int_{\partial \Omega \Omega \Gamma} \hat{\mathbf{q}}_{\mathbf{q}^{-}} \mathbf{q}_{\mathbf{q}^{+}}^{-} \phi_{r} \phi_{m} \vec{n} \mathrm{~d} S\right) \Delta \mathbf{q}_{r}^{N} \\
& +\left(\int_{\partial \Omega \backslash \Gamma} \hat{\mathbf{q}}_{\mathbf{q}^{-}} \phi_{r}^{-} \phi_{m} \vec{n} \mathrm{~d} S\right) \Delta \mathbf{q}_{r}^{N} \\
& -\int_{\Omega} \mathbf{q}_{0}^{N} \vec{\nabla} \phi_{m} \mathrm{~d} \Omega+\int_{\partial \Omega} \hat{\mathbf{q}}_{0} \phi_{m} \vec{n} \mathrm{~d} S .
\end{aligned}
$$

Since in all classical viscous numerical fluxes [6] $\hat{\mathbf{q}}$ is linear with respect to the 
solution on both sides of the interface, $\mathbf{q}^{+}$and $\mathbf{q}^{-},(5.42)$ can be reduced to

$$
\begin{aligned}
& \left(\int_{\Omega} \phi_{r} \phi_{m} \mathrm{~d} \Omega\right) \stackrel{\leftrightarrow}{\mathbf{g}}_{r}^{N}= \\
& \left(-\int_{\Omega} \phi_{r} \vec{\nabla} \phi_{m} \mathrm{~d} \Omega+\int_{\partial \Omega} \hat{\mathbf{q}}_{\mathbf{q}^{+}} \phi_{r} \phi_{m} \vec{n} \mathrm{~d} S+\int_{\partial \Omega \cap \Gamma} \hat{\mathbf{q}}_{\mathbf{q}^{-}} \mathbf{q}_{\mathbf{q}^{+}}^{-} \phi_{r} \phi_{m} \vec{n} \mathrm{~d} S\right) \underline{\mathbf{q}_{r}^{N}} \\
& +\left(\int_{\partial \Omega \backslash \Gamma} \hat{\mathbf{q}}_{\mathbf{q}^{-}} \phi_{r}^{-} \phi_{m} \vec{n} \mathrm{~d} S\right) \underline{\underline{\mathbf{q}_{r}^{N}}} .
\end{aligned}
$$

It is possible to write (5.43) in matrix form as

$$
\underline{\mathbf{M}} \stackrel{\leftrightarrow}{\mathbf{G}}^{N}=\underline{\hat{\mathbf{A}}}^{g} \mathbf{Q}^{N}+\underline{\hat{\mathbf{A}}}^{g} \mathbf{Q}^{\underline{N}},
$$

or similarly,

$$
\stackrel{\leftrightarrow}{\mathbf{G}}^{N}=\underline{\mathbf{A}}^{g} \mathbf{Q}^{N}+\underline{\underline{\mathbf{A}}}^{g} \mathbf{Q}^{N}
$$

where $\underline{A}^{g}$ is a third order tensor composed by a square $\mathrm{NDOF}^{e} \times \mathrm{NDOF}^{e}$ matrix in each spatial direction, and $\underline{\underline{A}}^{g}$ is a third order tensor composed by rectangular matrices in each spatial direction.

Using (5.45), (5.40) can be rewritten simply as

$$
\underline{\mathbf{A}}^{v} \Delta \mathbf{Q}^{N}+\underline{\mathbf{A}}^{v} \Delta \mathbf{Q}^{N}=\mathbf{B}^{v}
$$

where

$$
\begin{aligned}
& \underline{\mathbf{A}}^{v}=\underline{\mathbf{A}}^{v_{1}}+\underline{\mathbf{A}}^{v_{2}} \cdot \underline{\mathbf{A}}^{g}, \\
& \underline{\mathbf{A}}^{v}=\underline{\mathbf{A}}{ }^{v_{1}}+\underline{\mathbf{A}}^{v_{2}} \cdot \underline{\mathbf{A}} \underline{g} .
\end{aligned}
$$

\subsubsection{Viscous Numerical Fluxes}

The viscous numerical fluxes tend to have a simpler formulation than the advective numerical fluxes, so it is almost always easy to obtain the analytical expression for their associated Jacobian matrices. In the case of the symmetric IP we have

$$
\begin{aligned}
\hat{\mathbf{q}} & =\left\{\{\mathbf{q}\}=\frac{1}{2}\left(\mathbf{q}^{+}+\mathbf{q}^{-}\right),\right. \\
\hat{\mathbf{f}}^{v} & \left.=\left(\left\{\stackrel{\leftrightarrow}{\mathbf{f}}^{v}\right\}\right\}-\delta \llbracket \mathbf{q} \rrbracket\right) \cdot \vec{n} .
\end{aligned}
$$

Therefore, the Jacobian for the flux in the inner equation is linear:

$$
\hat{\mathbf{q}}_{\mathbf{q}^{+}}=\hat{\mathbf{q}}_{\mathbf{q}^{-}}=\frac{1}{2} \text {. }
$$


Additionally, for the outer equation we have

$$
\begin{aligned}
\hat{\mathbf{f}}_{\mathbf{q}^{+}}^{v} & =\frac{1}{2} \frac{\partial \overleftrightarrow{\mathbf{f}}^{v}\left(\mathbf{q}^{+}, \vec{\nabla} \mathbf{q}^{+}\right)}{\partial \mathbf{q}} \cdot \vec{n}-\delta \mathbf{I}_{n_{\text {cons }}} \\
& =\frac{1}{2} \underline{\mathbf{J}}^{v}\left(\mathbf{q}^{+}, \vec{\nabla} \mathbf{q}^{+}\right) \cdot \vec{n}-\delta \mathbf{I}_{n_{\text {cons }}},
\end{aligned}
$$

for the Jacobian with respect to $\mathbf{q}^{+}$. Similarly, the Jacobian with respect to $\mathbf{q}^{-}$is

$$
\begin{aligned}
\hat{\mathbf{f}}_{\mathbf{q}^{-}}^{v} & =\frac{1}{2} \frac{\partial \overleftrightarrow{\mathbf{f}}^{v}\left(\mathbf{q}^{-}, \vec{\nabla} \mathbf{q}^{-}\right)}{\partial \mathbf{q}} \cdot \vec{n}+\delta \mathbf{I}_{n_{\mathrm{cons}}} \\
& =\frac{1}{2} \underline{\underline{\mathbf{J}}}^{v}\left(\mathbf{q}^{-}, \vec{\nabla} \mathbf{q}^{-}\right) \cdot \vec{n}+\delta \mathbf{I}_{n_{\mathrm{cons}}}
\end{aligned}
$$

Finally, the Jacobians with respect to $\vec{\nabla} \mathbf{q}$ are

$$
\begin{aligned}
\hat{\mathbf{f}}_{\vec{\nabla} \mathbf{q}^{+}}^{v} & =\frac{1}{2} \frac{\partial \overleftrightarrow{\mathbf{f}^{v}}\left(\mathbf{q}^{+}, \vec{\nabla} \mathbf{q}^{+}\right)}{\partial(\vec{\nabla} \mathbf{q})} \cdot \vec{n} \\
& =\frac{1}{2} \underline{\underline{\underline{\mathbf{G}}}}\left(\mathbf{q}^{+}, \vec{\nabla} \mathbf{q}^{+}\right) \cdot \vec{n},
\end{aligned}
$$

and

$$
\begin{aligned}
\hat{\mathbf{f}}_{\vec{\nabla} \mathbf{q}^{-}}^{v} & =\frac{1}{2} \frac{\partial \overleftrightarrow{\mathbf{f}}^{v}\left(\mathbf{q}^{-}, \vec{\nabla} \mathbf{q}^{-}\right)}{\partial(\vec{\nabla} \mathbf{q})} \cdot \vec{n} \\
& =\frac{1}{2} \underline{\underline{\underline{\mathbf{G}}}}\left(\mathbf{q}^{-}, \vec{\nabla} \mathbf{q}^{-}\right) \cdot \vec{n} .
\end{aligned}
$$

\subsection{Performance Comparison of the Jacobian Computation Methods}

In this section, we present a comparison of the computational cost to compute and store the Jacobian matrix with the three methods that are described in this chapter: the numerical Jacobian, the numerical Jacobian that uses a coloring algorithm, and the analytical Jacobian. The computational cost is assessed in the evaluation of the Navier-Stokes Jacobian matrix for meshes with one to $25^{3}$ elements and different polynomial orders. The initial condition is the one used for the simulation of a Taylor-Green vortex (detailed in Section C.1.2).

Figure 5.6 shows a comparison of the computation time of the Jacobian (obtained sequentially) as a function of the number of degrees of freedom for the three methods. The analytical Jacobian computation is the most efficient method to compute the Jacobian, except for very small problems (with NDOF $<10$ ), where it is outperformed by the two numerical Jacobian methods. In this example, the 
analytical Jacobian computation time is up to four orders of magnitude lower than the time needed to compute the Jacobian numerically, and more than one order of magnitude lower than the Jacobian computation time that is obtained when using the coloring algorithm.

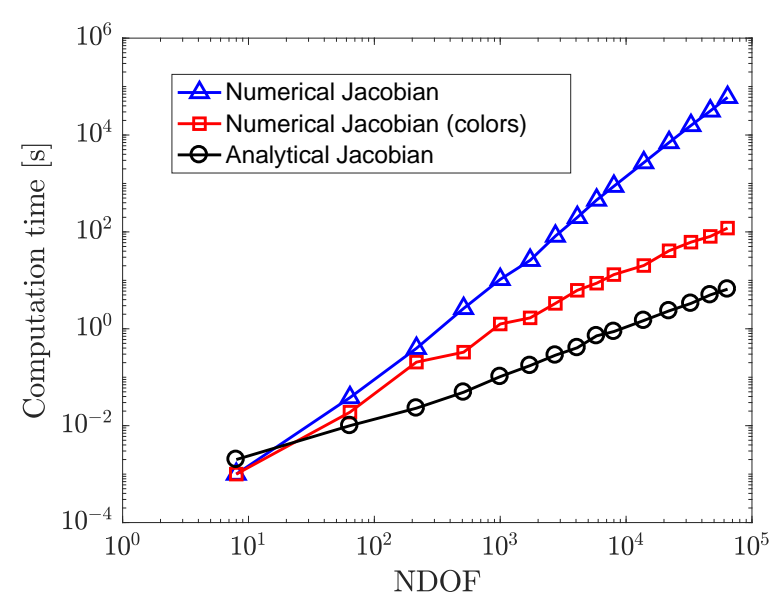

Figure 5.6: Sequential Jacobian computation times for the different techniques.

\subsection{Final Remarks}

In this chapter, several strategies for computing the Jacobian matrix were explained. The numerical Jacobian, based on a finite difference, is the easiest to formulate and implement, but has two main disadvantages: it is highly sensitive to round-off errors and is very expensive to compute. The second disadvantage can be alleviated to a great extent by the introduction of the coloring graph algorithm. Notwithstanding, the sensitivity to round-off errors remains and the Jacobian computation times can still be very long for the coloring Jacobians. Among the presented methods, the analytical computation of the Jacobian is the most complex from the derivation and implementation point of view, but it can provide shorter computation times and increased accuracy.

Jacobian-free methods were presented as an alternative to computing and storing the Jacobian matrix. These methods introduce a number of advantages, such as lower storage requirements and shorter computation times, but have the main drawback that the choice of linear solvers and preconditioners is somewhat limited. As a matter of fact, the linear solver strategy that is presented in Chapter 6 is only usable when the Jacobian matrix is computed.

Between the two most commonly used parallelization techniques (shared and distributed memory parallelization), the implementation of the Jacobian computation routines is slightly more complicated with distributed memory (e.g. through 
MPI) than with shared memory (e.g. through OpenMP). However, it is important to point out that distributed memory parallelization offers several advantages over shared memory in implicit discretizations. First and foremost, as in explicit discretizations, the currently available hardware requires distributed memory parallelization for medium to large problems. Second, most standard sparse formats (e.g. CSR) store the matrix values in continuous arrays; therefore, the use of shared memory may lead to false sharing issues, which are most commonly dealt with with critical regions, which are in turn very inefficient from the computational point of view.

Because of the problems of shared memory parallelization, many broadly used libraries, such as PETSc [1] and HYPRE [60], only support distributed memory parallelization (MPI). Furthermore, when the problem size is increased, the size of the solution vectors and the matrix becomes so big that, if one uses shared memory parallelization, they cannot be allocated in stack memory. As a result, they have to be allocated in heap memory, which further affects the performance of the code. In the current implementation in HORSES3D, pure MPI parallelization provides better scalability properties and storage management than OpenMP for medium-to-large problems.

A technique called Automatic Differentiation (AD) has become a popular alternative to numerically or analytically computing the Jacobian matrices [85]. AD tries to solve two main issues of traditional methods: numerical Jacobians are very expensive to compute and the derivation of analytical Jacobians is error-prone and complicated to implement. Therefore, AD relies on a external tool for the implementation of the Jacobian routines. The only thing that the user needs to provide are the routines for the computation of the explicit nonlinear operators. Based on that and on the chain rule, the AD tool generates the code that computes the Jacobian matrix. As is natural to expect, the efficiency of the generated code depends on the quality of the external tool. Furthermore, a difficulty of AD-generated codes is that they are hard to read and follow, and that care must be taken in order to incorporate adequate sparse matrix formats. All in all, analytical Jacobians can still be more computer-efficient than $\mathrm{AD}$ if programmed carefully.

Because of the reasons exposed above, in this thesis analytical Jacobians are used for the numerical examples where Jacobian matrices are needed. It is important to remark that, although it was stated that the Jacobian matrix must be evaluated with the solution of the last iteration, the usual practice is to freeze it (use the same matrix in several iterations) and only recompute it when the convergence rate degrades below a certain threshold. Namely, the computational cost of the Jacobian computation is usually large and it is not necessary to recompute the Jacobian if the solution changes little. 


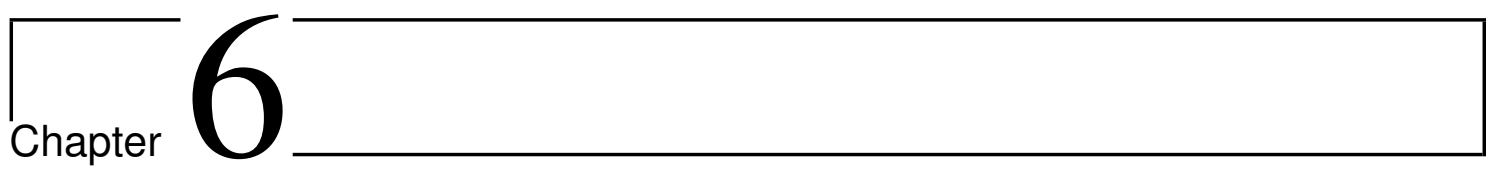

\section{A Static-Condensation Method for the GL-DGSEM}

\section{Contents}

6.1 Introduction . . . . . . . . . . . . . . . 73

6.2 Background: The Static-Condensation Method . . . . . . . 76

6.2.1 Continuous Galerkin Methods . . . . . . . . . . . . . 77

6.2.2 Discontinuous Galerkin Methods . . . . . . . . . . . . 77

6.3 Jacobian Sparsity Patterns for the DGSEM . . . . . . . . . 79

6.3 .1 Advective Terms . . . . . . . . . . . . . . . 80

6.3 .2 Diffusive Terms . . . . . . . . . . . . . . . 82

6.4 Static-Condensation for the GL-DGSEM . . . . . . . . . . 85

6.5 Numerical Example . . . . . . . . . . . . . . . . . 87

6.6 Final Remarks $\ldots \ldots \ldots \ldots \ldots \ldots$

\subsection{Introduction}

In this chapter, a static-condensation method for time-implicit discretizations of the Discontinuous Galerkin Spectral Element Method on Gauss-Lobatto points (GLDGSEM) is presented. The method here exposed is a novel contribution, as it is not an HDG method and, to the author's knowledge, it has not been attempted before. We show that it is possible to directly organize the linear systems that result from the implicit time-integration of the GL-DGSEM as a Schur complement problem, which can be efficiently solved using static condensation. The static-condensation method presented here can be understood as one of many advantages of the GLDGSEM over its version on Gauss nodes (the G-DGSEM).

As was discussed in Chapter 2, the standard choices for the position of the nodes of the DGSEM are the Gauss and the Gauss-Lobatto quadrature points. This makes 
the DGSEM a so-called collocation method, where the solution is stored in the position where it is needed to numerically evaluate the volume integrals. The collocation property gives the DGSEM a computational advantage over other modal and nodal DG methods, where the conservative variables and the fluxes must be evaluated on the quadrature nodes as an additional step.

The GL-DGSEM provides several advantages over the G-DGSEM. The first advantage is that, in the former method, the solution is stored where it is needed for the evaluation of the surface integrals, whereas it is not in the G-DGSEM. This makes the GL-DGSEM computationally cheaper and easier to implement since the solution does not have to be interpolated to the element boundaries, as in the GDGSEM. In addition, it has been shown that the GL-DGSEM allows taking larger time steps than the G-DGSEM because of the inherent spectrum of the spatial operator [75]. Furthermore, the GL-DGSEM can be used to unify certain diffusive numerical fluxes as it has been shown that the Bassi-Rebay 1 scheme is a special case of the symetric Interior Penalty method when Gauss-Lobatto nodes are used [138].

The only disadvantage of the Gauss-Lobatto quadrature is that it is not as accurate as the Gauss quadrature: The numerical integration is only exact for polynomials of order up to $2 N-1$ when using Gauss-Lobatto, whereas it is exact for polynomials of order up to $2 N+1$ when using Gauss (see Section 2.2). This induces aliasing errors that can trigger instabilities, which in turn can make the solution blow up, specially at high Reynolds numbers [135]. The traditional DGSEM on Gauss nodes also suffers from those instabilities, but they appear sooner when Gauss-Lobatto nodes are used [75, 135].

The common solution to aliasing-driven instabilities is to increase the number of quadrature points in order to reduce (or eliminate) the aliasing errors, in an approach that is called polynomial dealiasing or over-integration. The overintegration strategy increases the computational cost since collocation is no longer possible. Moreover, an exact numerical integration is not always achievable, no matter the number of quadrature points. For some systems of equations, like the compressible Euler or Navier-Stokes, the fluxes are not polynomials but ratios of polynomials [80].

It has also been shown that the aliasing driven instabilities can be eliminated with the use of a split form of the governing equations [69, 118]. This can be achieved while keeping discrete conservation properties, if the discretization scheme satisfies the summation-by-parts simultaneous-approximation-term (SBP-SAT) property [69]. It has been shown that the GL-DGSEM satisfies the SBP-SAT property [77] and that stable split forms can be formulated for the Burgers equation [77], the compressible Navier-Stokes equations [80], the MHD equations [26], the CahnHilliard equation [136], the incompressible Navier-Stokes equations [137], etc. The split form has a dealiasing effect that makes the GL-DGSEM provably stable (something that is not achievable with the G-DGSEM or polynomial dealiasing), while still being computationally cheaper than over-integrated methods and yield- 
ing comparable results [204]. All these studies point out that the GL-DGSEM has a numerical superiority over the traditional G-DGSEM, and therefore we retain Gauss-Lobatto quadrature rules.

Static condensation [87] is a technique to reduce the size of linear systems. It has been widely used in the continuous Galerkin (CG) community to solve timeimplicit discretizations [53, 104, 199]. However, because of the way the degrees of freedom of different elements are coupled in DG discretizations, static condensation is not directly applicable to general DG methods in an efficient manner.

To the author's knowledge, two techniques have been developed to efficiently apply static condensation to DG schemes. The first technique was developed by Sherwin et al. [188], and uses specially tailored basis functions with which it is possible to statically condense the linear system that arises from the time-implicit DG discretization. Unfortunately, the new basis functions are neither orthogonal nor tensor-product expansions. Therefore, some properties are lost, like the existence of diagonal mass matrices, Kroenecker multiplication matrices, the ability to perform anisotropic $p$-adaptation, among others.

The second technique is the hybridizable DG (or HDG) method, which was developed by Carrero and Cockburn $[43,47]$. This method expands the linear system to include the numerical trace of the solution as new unknown, to statically condense it around this new variable. This technique has been proved to be computationally efficient $[152,155]$, but it imposes certain constraints on the numerical fluxes of the DG method, such as the need for the elliptic fluxes to be adjoint consistent and compact [47], and the requirement for the numerical fluxes of nonlinear advection-diffusion equations to have a specific mathematical form [152, 155]. As a result, not all the known Riemann solvers can be classified as suitable for HDG.

In this chapter, we show that the static-condensation method can be directly applied to the time-implicit GL-DGSEM in an efficient manner without the strong constraints of previous implementations. Namely, we keep tensor-product orthogonal bases in a method that can be used with any choice of the numerical flux functions, with the only requirement for the viscous numerical flux one to be compact. We show, by means of a numerical experiment, that the developed method provides significant speed-ups when compared to the time-explicit GL-DGSEM and the not statically condensed time-implicit GL-DGSEM. Therefore, this investigation reveals a further advantage of Gauss-Lobatto quadratures over traditional Gauss quadratures in the DGSEM.

The chapter is organized as follows. Section 6.2 provides an overview of the static-condensation method and the way it has been implemented in continuous and discontinuous Galerkin methods. Section 6.3 contains an analysis of the sparsity patterns of DGSEM methods, where we demonstrate that static condensation can be efficiently applied when Gauss-Lobatto nodes are used. Next, in Section 6.4, we detail the implementation of the statically-condensed GL-DGSEM that is used in 
this work. Finally, in Section 6.5, the method is tested for solving the compressible Navier-Stokes equations.

\subsection{Background: The Static-Condensation Method}

The static-condensation method, or Guyan reduction [87], is a well-known technique to reduce the size of linear systems that can be written in blocks as

$$
\left[\begin{array}{ll}
\underline{\mathbf{B}} & \underline{\mathbf{C}} \\
\underline{\mathbf{E}} & \underline{6}
\end{array}\right]\left[\begin{array}{l}
\mathbf{X}_{1} \\
\mathbf{X}_{2}
\end{array}\right]=\left[\begin{array}{l}
\mathbf{F}_{1} \\
\mathbf{F}_{2}
\end{array}\right]
$$

where $\underline{\mathbf{B}} \in \mathbb{R}^{n_{1} \times n_{1}}, \underline{\mathbf{C}} \in \mathbb{R}^{n_{1} \times n_{2}}, \underline{\mathbf{D}} \in \mathbb{R}^{n_{2} \times n_{1}}$, and $\underline{\mathbf{E}} \in \mathbb{R}^{n_{2} \times n_{2}}$.

We start by performing block Gauss elimination, which can be summarized as multiplying the system (6.1) on the left by the matrix

$$
\left[\begin{array}{cc}
\underline{\mathrm{I}} & -\mathrm{CE}^{-1} \\
\mathbf{0} & \underline{\mathrm{I}}
\end{array}\right]
$$

to obtain

$$
\left[\begin{array}{cc}
\underline{\mathbf{B}}-\underline{\mathbf{C E}}^{-1} \underline{\mathbf{D}} & \mathbf{0} \\
\underline{\mathbf{D}} & \underline{\mathbf{E}}
\end{array}\right]\left[\begin{array}{l}
\mathbf{X}_{1} \\
\mathbf{X}_{2}
\end{array}\right]=\left[\begin{array}{c}
\mathbf{F}_{1}-\underline{\mathbf{C E}}^{-1} \mathbf{F}_{2} \\
\mathbf{F}_{2}
\end{array}\right]
$$

Hence, the the linear system for $\mathbf{X}_{1}$ is decoupled from the rest of the system with a block of zeros in the upper off-diagonal. As a result, the original system can be solved in two steps:

1. Solve the statically-condensed system for $\mathbf{X}_{1}$,

$$
\left[\underline{B}-\underline{C E}^{-1} \underline{D}\right] X_{1}=F_{1}-\underline{C E}^{-1} \mathbf{F}_{2}
$$

where the condensed matrix is also known as the Schur complement of the original global matrix.

2. Compute $\mathbf{X}_{2}$ as a function of $\mathbf{X}_{1}$,

$$
\mathbf{X}_{2}=\underline{\mathbf{E}}^{-1}\left(\mathbf{F}_{2}-\underline{\mathbf{D}} \mathbf{X}_{1}\right) .
$$

This approach is computationally efficient if $n_{1}$ is small and the matrix $\mathbf{E}$ is easily invertible. In fact, the action of $\underline{E}^{-1}$ on a vector is needed in equation $(\overline{6} .4)$ to construct the statically condensed system matrix ( $n_{1}$ times, i.e. the number of columns of $\underline{D}$ ) and to construct the statically-condensed RHS (one time), and in equation (6.5) to recover $\mathbf{X}_{2}$ (one time).

The static-condensation method has been applied to time-implicit Continuous Galerkin (CG) [53, 104, 199] and Discontinuous Galerkin (DG) [43, 47, 188] methods, where the linear system is of the form (see Chapter 5 for the detailed derivation for DG methods):

$$
\underline{\mathrm{A}} \mathbf{Q}=\mathrm{B} .
$$


Note that the delta symbol is omitted for readability, $\Delta \mathbf{Q} \leftarrow \mathbf{Q}$, which does not necessarily imply that we are dealing with linear fluxes.

In what follows, we present a brief description of the state-of-the-art implementations of the static-condensation method for CG and DG, and then we show how it can be applied to the GL-DGSEM in an efficient manner.

\subsubsection{Continuous Galerkin Methods}

Static condensation was first used to reduce the size of the linear system that results from time-implicit Finite Element discretizations by Fraeijs in 1965 [53]. In general, in medium-to-high order $(N \geq 2)$ continuous Galerkin (CG) discretizations, it is easy to reorganize the linear system (6.6) as

$$
\left[\begin{array}{ll}
\underline{\mathbf{A}}_{b b} & \mathbf{\mathbf { A }}_{i b} \\
\underline{\mathbf{A}}_{b i} & \underline{\mathbf{A}}_{i i}
\end{array}\right]\left[\begin{array}{l}
\mathbf{Q}_{b} \\
\mathbf{Q}_{i}
\end{array}\right]=\left[\begin{array}{l}
\mathbf{B}_{b} \\
\mathbf{B}_{i}
\end{array}\right],
$$

where $\mathbf{Q}_{b}$ is the solution on the degrees of freedom that sit on the element boundaries (interfaces), and $\mathbf{Q}_{i}$ is the solution on the inner degrees of freedom. Moreover, $\underline{\mathbf{A}}_{b b}$ is the boundary-to-boundary matrix, $\underline{\mathbf{A}}_{i b}$ is the interior-to-boundary matrix, $\underline{\mathbf{A}}_{b i}$ is the boundary-to-interior matrix, and $\underline{\mathbf{A}}_{i i}$ is the interior-to-interior matrix.

Note that system (6.7) is equivalent to system (6.1) for

$$
\left[\begin{array}{ll}
\underline{\mathbf{B}} & \underline{\mathbf{C}} \\
\underline{\mathbf{D}} & \underline{\mathbf{E}}
\end{array}\right]=\left[\begin{array}{ll}
\underline{\mathbf{A}}_{b b} & \underline{\mathbf{A}}_{i b} \\
\underline{\mathbf{A}}_{b i} & \underline{\mathbf{A}}_{i i}
\end{array}\right],\left[\begin{array}{l}
\mathbf{X}_{1} \\
\mathbf{X}_{2}
\end{array}\right]=\left[\begin{array}{l}
\mathbf{Q}_{b} \\
\mathbf{Q}_{i}
\end{array}\right],\left[\begin{array}{l}
\mathbf{F}_{1} \\
\mathbf{F}_{2}
\end{array}\right]=\left[\begin{array}{l}
\mathbf{B}_{b} \\
\mathbf{B}_{i}
\end{array}\right],
$$

and it can be solved using the same two-step procedure. Furthermore, since in CG the coupling between elements occurs only through the (shared) degrees of freedom on element interfaces, the matrix $\underline{\mathbf{A}}_{i i}$ has a block diagonal structure, which makes it easy to invert in a local manner.

This approach has been used by Karniadakis and Sherwin [104] and Vos et al. [199] for high-order CG methods, who have shown that the computational efficiency is increased when the order of the approximation $(N)$ is increased because the relative size of the condensed system, $n_{1} /\left(n_{1}+n_{2}\right)$, decreases with $N$.

\subsubsection{Discontinuous Galerkin Methods}

We now detail how static condensation has been used with high-order DG methods. Unlike CG methods, DG methods may couple all the degrees of freedom of an element with the degrees of freedom of its neighbors (see (5.22) and (5.37)), or neighbors of neighbors (if a non-compact DG method is used), through the numerical flux functions. As a result, the static-condensation method is in general not directly applicable.

The first implementation of a static-condensation DG scheme was presented by Sherwin et al. [188], who were able to make a modal DG scheme suitable for 
static condensation by using $C^{0}$-type expansions for the basis functions on element boundaries and bubble functions for the inner modes. This choice of basis resembles the one used in $p$-FEM, a type of continuous Galerkin methods. Consequently, the linear system that results from the implicit time-integration of the modified DG scheme can also be arranged as the system (6.7), where the matrix $\underline{\mathbf{A}}_{i i}$ is also block diagonal. This proved to be advantageous since the statically condensed system was shown to be not only smaller in size but also cheap-to-compute and better conditioned than the global system.

Sherwin et al. [188] allege that an additional advantage of their statistically condensable DG is that the boundary conditions can be imposed through global lifting, as is in continuous Galerkin methods, hence reducing the number of degrees of freedom of the problem. This is useful to treat elliptic problems but it may need stabilization for hyperbolic equations.

The only drawback of Sherwin's approach is that the new specially tailored basis functions are neither orthogonal expansions nor tensor-product bases. Therefore, several advantages of such basis functions cannot be kept, such as the existence of diagonal mass matrices, sparser Jacobians, the possibility to evaluate the anisotropic truncation error estimator of Chapter 8, the ability to perform anisotropic $p$-adaptation, among others.

Another approach to perform static condensation in DG methods was developed simultaneously and independently from Sherwin's approach by Carrero and Cockburn et al. [43, 47] and is known as the Hybridizable Discontinuous Galerkin (HDG) method. This method imposes no restrictions on the choice of basis functions and has gained increased popularity in recent years [61, 189].

The HDG method expands the original DG system with a new unknown variable that only lives on the mesh skeleton, $\lambda$ (typically the numerical trace of the solution, $\lambda=\hat{\mathbf{q}})$, with which it is possible to statically condense it. The expanded system is

$$
\left[\begin{array}{ll}
\underline{B} & \underline{\mathbf{C}} \\
\underline{\mathbf{D}} & \underline{\mathrm{A}}_{B}
\end{array}\right]\left[\begin{array}{l}
\Lambda \\
\mathbf{Q}
\end{array}\right]=\left[\begin{array}{c}
\mathbf{F}_{1} \\
\mathbf{B}
\end{array}\right]
$$

where $\underline{\mathbf{A}}_{B}$ is a matrix formed by the diagonal blocks of matrix $\underline{\mathbf{A}}, \boldsymbol{\Lambda}$ is the sampled version of $\boldsymbol{\lambda}$, and $\underline{\mathbf{B}}, \underline{\mathbf{C}}, \underline{\mathbf{D}}$ and $\mathbf{F}_{1}$ are additional terms that contain the scattered information of the off-diagonal blocks of $\underline{\mathbf{A}}$. A similar approach is done by Petersen [158] for a space-time DG, where $\Lambda$ are Lagrange multipliers.

Note that we kept almost the same notation of (6.6) in (6.9), but some variables where changed by the names they usually have in the HDG community. Therefore we have

$$
\left[\begin{array}{ll}
\underline{\mathbf{B}} & \underline{\mathbf{C}} \\
\underline{\mathbf{E}} & \underline{\mathbf{E}}
\end{array}\right]=\left[\begin{array}{ll}
\underline{\mathbf{B}} & \underline{\mathbf{C}} \\
\underline{\mathbf{D}} & \underline{\mathbf{A}}_{B}
\end{array}\right],\left[\begin{array}{l}
\mathbf{X}_{1} \\
\mathbf{X}_{2}
\end{array}\right]=\left[\begin{array}{l}
\Lambda \\
\mathbf{Q}
\end{array}\right],\left[\begin{array}{l}
\mathbf{F}_{1} \\
\mathbf{F}_{2}
\end{array}\right]=\left[\begin{array}{c}
\mathbf{F}_{1} \\
\mathbf{B}
\end{array}\right] .
$$

The first formulations of the HDG method [43, 47] dealt with linear elliptic problems and imposed particular conditions, such as the requirement on the viscous numerical fluxes to be adjoint-consistent (see for example [6]). When solving nonlinear conservation laws, additional constraints are imposed for a DG method 
to be hybridizable with $\lambda=\hat{\mathbf{q}}$. For example, the numerical flux is restricted to the form $[152,155]$

$$
\hat{\mathbf{f}}=\stackrel{\leftrightarrow}{\mathbf{f}}(\hat{\mathbf{q}}) \cdot \vec{n}+\underline{\mathbf{S}}\left(\mathbf{q}^{N}, \hat{\mathbf{q}}\right)\left(\mathbf{q}^{N}-\hat{\mathbf{q}}\right)
$$

where $\mathbf{q}^{N}$ is the solution on the analyzed element, $\hat{\mathbf{q}}$ is the numerical trace of it, and $\underline{\mathbf{S}}$ is a stabilizing function. Some broadly used numerical flux functions, such as the Lax-Friedrichs flux, can be expressed in this form, but others (e.g. Roe) cannot.

Without the constraint (6.11), it would not be possible to condense the system as a function of $\hat{\mathbf{Q}}$. However, we would like to point out that, in purely advective nonlinear conservation laws, it is possible to use $\boldsymbol{\lambda}=\hat{\mathbf{f}}^{a}$ and ignore the restriction (6.11).

\subsection{Jacobian Sparsity Patterns for the DGSEM}

In this section, an analysis of the sparsity patterns of the matrices that result of timeimplicit DGSEM discretizations is performed. From this analysis, it follows that the linear systems for the DGSEM on Gauss-Lobatto points can be directly organized as (6.7), where $\underline{A}_{i i}$ is a block-diagonal matrix. Hence, the static-condensation method can be directly and efficiently applied to the GL-DGSEM. As discussed in Chapter 5, we are interested in compact viscous fluxes since they translate in sparser matrices that need less storage and whose matrix operations require fewer floating point operations.

Following the methodology of Chapter 5, we first present the analysis for the advective terms and then for the viscous terms. Furthermore, the sparsity patterns of a seven-element 1D discretization of order $N=9$ (Figure 6.1(a)) and an eightelement 3D discretization of order $N=3$ (Figure 6.1(b)) will be illustrated in this section in order to simplify the analysis.

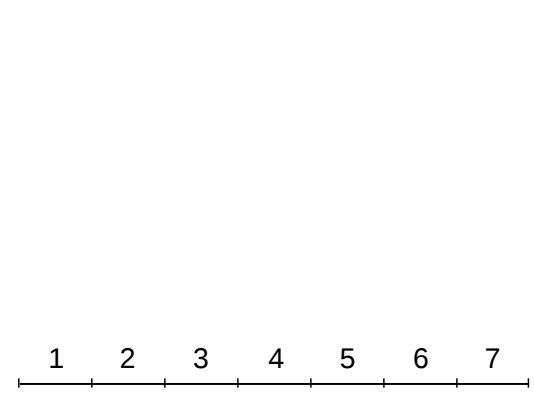

(a) $1 \mathrm{D}$ mesh

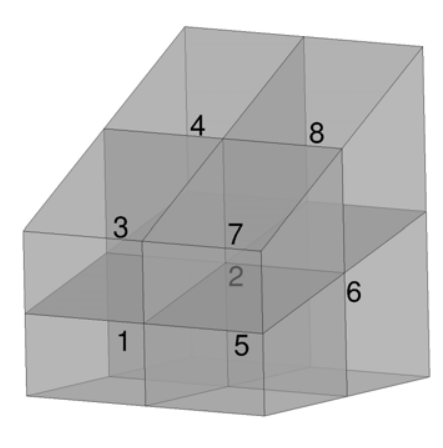

(b) 3D mesh

Figure 6.1: Meshes analyzed for sparsity patterns showing the element numbering. 


\subsubsection{Advective Terms}

The entries of the Jacobian matrix are presented in (5.22) for a standard timeimplicit DG discretization of an advective conservation law. When using the DGSEM, the volume and surface integrals are replaced by numerical quadratures and the basis functions are nothing but tensor-product expansions of the Lagrange interpolating polynomials. Therefore, (5.22) can be rewritten as

$$
\begin{array}{r}
J_{j} w_{j} \partial_{t} \mathbf{q}_{j}^{N} \\
+\left(-\int_{\Omega}^{N}\left(\underline{\mathbf{J}}^{a} \phi\right)_{r} \cdot \vec{\nabla} \phi_{j} \mathrm{~d} \Omega+\int_{\partial \Omega}^{N} \hat{\mathbf{f}}_{\mathbf{q}^{+}}^{a} \phi_{r} \phi_{j} \mathrm{~d} S+\int_{\partial \Omega \cap \Gamma}^{N} \hat{\mathbf{f}}_{\mathbf{q}^{-}}^{a} \mathbf{q}_{\mathbf{q}^{+}}^{-} \phi_{r} \phi_{j} \mathrm{~d} S\right) \Delta \mathbf{q}_{r}^{N} \\
+\left(\int_{\partial \Omega \backslash \Gamma}^{N} \hat{\mathbf{f}}_{\mathbf{q}^{-}}^{a} \phi_{r}^{-} \phi_{j} \mathrm{~d} S\right) \Delta \mathbf{q}_{r}^{N}=\hat{\mathbf{b}}_{j}^{a} .
\end{array}
$$

The red underlined term in (6.12) corresponds to the off-diagonal blocks of the Jacobian matrix. This term is guaranteed to be zero if the basis functions, $\phi_{j}$ or $\phi_{r}^{-}$, are zero on the element interface.

If one uses the DGSEM, the basis functions are tensor-product reconstructions of the Lagrange interpolating polynomials on Gauss or Gauss-Lobatto nodes (there is one basis function for each node/degree of freedom). Furthermore, as explained in Chapter 2, if one uses Gauss nodes, all basis functions take nonzero values on the element interfaces. Therefore, $\phi_{j}$ and $\phi_{r}^{+}$always contribute to the underlined surface integral (for any $j$ and $r$ ). On the other hand, if one uses Gauss-Lobatto nodes, only the basis functions that correspond to interface degrees of freedom take nonzero values on the element interfaces. Therefore, $\phi_{j}$ and $\phi_{r}^{+}$only contribute to the underlined surface integral if $j$ and $r$ are degrees of freedom that sit on the element boundary. See Figure 2.2 for details on how the basis functions look like on the two node distributions.

The difference between the basis functions on Gauss and Gauss-Lobatto nodes causes different matrix sparsity patterns for the two node distributions, which are illustrated in Figure 6.2 for the 1D mesh of Figure 6.1(a) and a scalar $\left(n_{\text {cons }}=1\right)$ advection equation. Note that in the 1D DGSEM with Gauss nodes, all the degrees of freedom of a given element are coupled with the degrees of freedom of a neighbor element through entries in the corresponding off-diagonal block. Contrarily, in the DGSEM with Gauss-Lobatto nodes, only the boundary degrees of freedom are coupled through entries on the off-diagonal block. As a result, the 1D GL-DGSEM matrix is almost block-diagonal (there is only one entry in each off-diagonal block). Consequently, it is possible to move up the rows and left the columns that correspond to boundary degrees of freedom (reorganize the linear system) in order to obtain an equivalent linear system with the structure of (6.7), where $A_{i i}$ is a blockdiagonal matrix.

Figure 6.3 shows that a similar behavior is observed for the 3D mesh of Figure 6.1(b). In this context, the sparsity patterns are also shown for a scalar PDE, where 


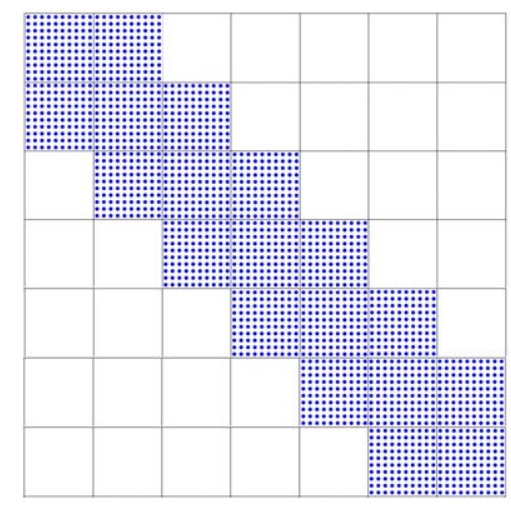

(a) DGSEM on Gauss nodes

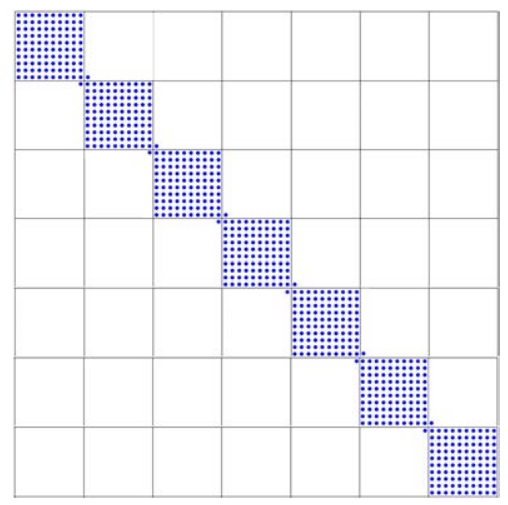

(b) DGSEM on Gauss-Lobatto nodes

Figure 6.2: Sparsity patterns of system matrices for 1D scalar advective equations.

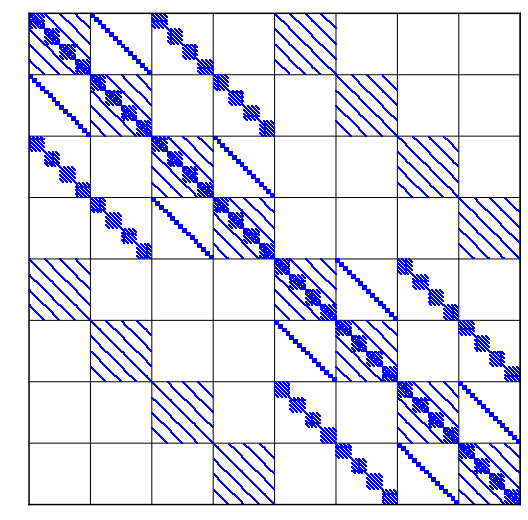

(a) DGSEM on Gauss nodes

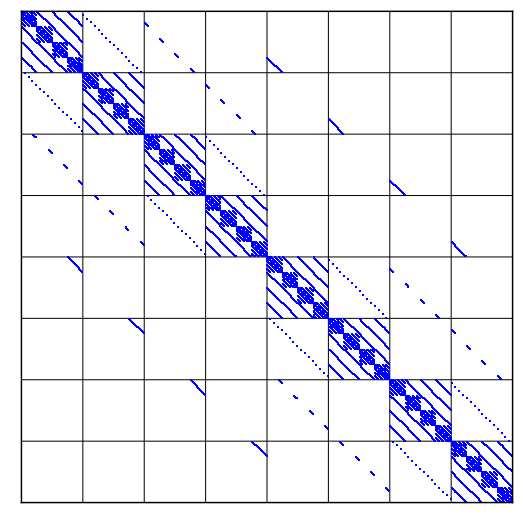

(b) DGSEM on Gauss-Lobatto nodes

Figure 6.3: Sparsity patterns of system matrices for advective 3D single-equation discretizations. 
each pixel is an entry of the Jacobian matrix. In multi-equation PDEs, the sparsity pattern is very similar, but each pixel would contain an $n_{\text {cons }} \times n_{\text {cons }}$ matrix that is not necessarily dense. In 3D, the diagonal and off-diagonal blocks are no longer dense because of the tensor-product basis expansions. This can be exploited to reduce the storage requirements, specially for very high orders $(N>3)$.

Furthermore, in the 3D DGSEM with Gauss nodes all the degrees of freedom of a given element are coupled (through the off-diagonal block) with some degrees of freedom of the neighbor elements in a way that makes it impossible to reorganize the system as (6.7) with $A_{i i}$ as a block-diagonal matrix. However, when GaussLobatto nodes are used this is possible since only the boundary degrees of freedom are coupled with other boundary degrees of freedom.

\subsubsection{Diffusive Terms}

In the presence of diffusive terms in the PDE, and a compact viscous numerical flux, the DG Jacobian contains additional terms, as can be interpreted from (5.37), here rewritten with the DGSEM considerations,

$$
\begin{aligned}
& \left(\int_{\Omega}^{N}\left(\underline{\mathbf{J}}^{v} \phi\right)_{r} \cdot \vec{\nabla} \phi_{j} \mathrm{~d} \Omega\right) \Delta \mathbf{q}_{r}^{N}+\left(\int_{\Omega}^{N}(\underline{\underline{\underline{G}}} \phi)_{m} \cdot \vec{\nabla} \phi_{j} \mathrm{~d} \Omega\right) \cdot \Delta \overleftrightarrow{\mathbf{g}}_{m}^{N} \\
& -\left(\int_{\partial \Omega \backslash \Gamma}^{N}\left(\hat{\mathbf{f}}_{\mathbf{q}^{+}}^{v} \phi_{r}+\hat{\mathbf{f}}_{\vec{\nabla} \mathbf{q}^{+}}^{v} \cdot \vec{\nabla} \phi_{r}\right) \phi_{j} \mathrm{~d} \Omega\right) \Delta \mathbf{q}_{r}^{N} \\
& -\left(\int_{\partial \Omega \cap \Gamma}^{N}\left(\frac{\partial \hat{\mathbf{f}}_{\Gamma}^{v}}{\partial \mathbf{q}^{+}} \phi_{r}+\frac{\partial \hat{\mathbf{f}}_{\Gamma}^{v}}{\partial \vec{\nabla} \mathbf{q}^{+}} \cdot \vec{\nabla} \phi_{r}\right) \phi_{j} \mathrm{~d} \Omega\right) \Delta \mathbf{q}_{r}^{N} \\
& -\left(\int_{\partial \Omega \backslash \Gamma}^{N}\left(\hat{\mathbf{f}}_{\mathbf{q}^{-}}^{v} \phi_{r}^{-}+\hat{\mathbf{f}}_{\vec{\nabla} \mathbf{q}^{-}}^{v} \vec{\nabla} \phi_{r}^{-}\right) \phi_{j} \mathrm{~d} \Omega\right) \Delta \mathbf{q}_{r}^{N}=\hat{\mathbf{b}}_{j}^{v},
\end{aligned}
$$

where the terms that contribute to the off-diagonal blocks are, again, red underlined.

The first underlined term of (6.13) contributes to an off-diagonal block through (5.43), which is here rewritten taking into account the DGSEM specific properties,

$$
\begin{array}{r}
J_{m} w_{m} \stackrel{\mathbf{g}}{m}=\left(-\int_{\Omega}^{N} \phi_{r} \vec{\nabla} \phi_{m} \mathrm{~d} \Omega+\int_{\partial \Omega}^{N} \hat{\mathbf{q}}_{\mathbf{q}^{+}} \phi_{r} \phi_{m} \vec{n} \mathrm{~d} S+\int_{\partial \Omega \cap \Gamma}^{N} \hat{\mathbf{q}}_{\mathbf{q}^{-}} \mathbf{q}_{\mathbf{q}^{+}}^{-} \phi_{r} \phi_{m} \vec{n} \mathrm{~d} S\right) \mathbf{q}_{r}^{N} \\
+\left(\int_{\partial \Omega \backslash \Gamma}^{N} \hat{\mathbf{q}}_{\mathbf{q}^{-}} \boldsymbol{\phi}_{r}^{-} \boldsymbol{\phi}_{m} \vec{n} \mathrm{~d} S\right) \mathbf{q}_{r}^{N} .
\end{array}
$$

Taking into account that the index $m$ in (6.13) is used in an Einstein summation convention, the off-diagonal term (ODT ${ }_{j r}$ ) that corresponds to the degree of freedom $j$ of the element $e$, and the degree of freedom $r$ of a certain neighbor element 
(i.e. the term that multiplies $\Delta \mathbf{q}_{r}^{N}$ ), can be written for the DGSEM as

$$
\begin{array}{r}
\mathrm{ODT}_{j r}=\sum_{m=1}^{\mathrm{NDOF}^{e}}\left[\frac{1}{J_{m} w_{m}}\left(\int_{\Omega}^{N} \underline{\underline{\underline{G}}}_{m} \phi_{m} \cdot \vec{\nabla} \phi_{j} \mathrm{~d} \Omega\right) \cdot\left(\int_{\partial \Omega \backslash \Gamma}^{N} \phi_{r}^{-} \phi_{m} \vec{n} \mathrm{~d} S\right)\right] \\
-\int_{\partial \Omega \backslash \Gamma}^{N}\left(\hat{\mathbf{f}}_{\mathbf{q}^{-}}^{v} \phi_{r}^{-}+\hat{\mathbf{f}}_{\vec{\nabla} \mathbf{q}^{-}}^{v} \vec{\nabla} \phi_{r}^{-}\right) \phi_{j} \mathrm{~d} \Omega .
\end{array}
$$

It is evident that (6.15) generates much denser off-diagonal blocks than the ones obtained for the advective terms. As a matter of fact, on a first sight one could think that static condensation is not applicable to the time-implicit GL-DGSEM when diffusive terms are present. However, it is, as can be observed in Figure 6.4, which shows the resulting sparsity patterns for the 1D mesh of Figure 6.1(a).

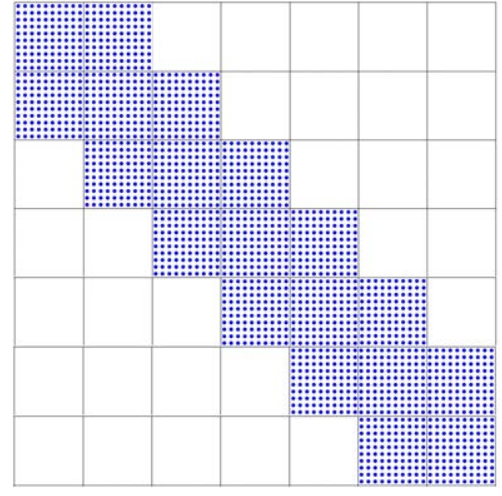

(a) DGSEM on Gauss nodes

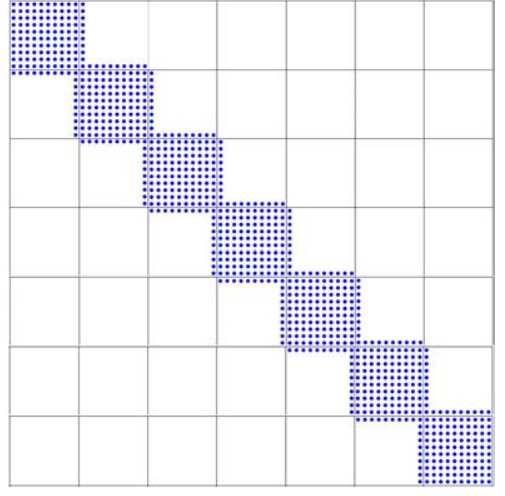

(b) DGSEM on Gauss-Lobatto nodes

Figure 6.4: Sparsity patterns of system matrices for advection-diffusion 1D singleequation discretizations.

The 1D sparsity pattern for the DGSEM on Gauss nodes is the same as in the advective case, but there are substantial differences when Gauss-Lobatto nodes are used. To begin with, the first term of (6.15) takes nonzero values for any degree of freedom $j$ of the analyzed element, if and only if $r$ corresponds to a boundary degree of freedom of a neighbor element, i.e. when $\phi_{r}^{-} \neq 0$. Moreover, the second term takes nonzero values for any $r$, if and only if $\phi_{j} \neq 0$, i.e. for a boundary degree of freedom of the analyzed element. As a result, a whole row and a whole column of each off-diagonal block of the 1D system matrix is filled with nonzero values. These blocks are indeed denser than in the advective case, but the static-condensation method can still be applied with the exact same row/column reordering.

The 3D discretization is more complex than the $1 \mathrm{D}$, but it retains similar properties. Figure 6.5 shows the sparsity pattern that results from the time-implicit DGSEM discretization of a scalar nonlinear advection-diffusion equation in the 3D 


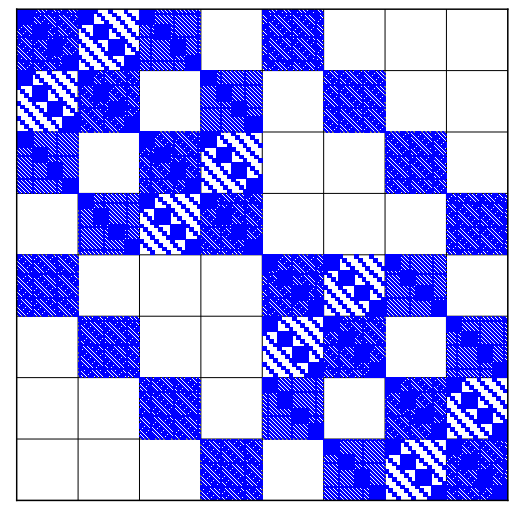

(a) DGSEM on Gauss nodes

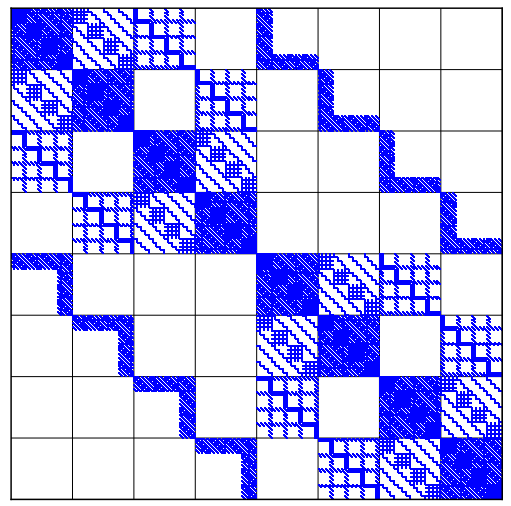

(b) DGSEM on Gauss-Lobatto nodes

Figure 6.5: Sparsity patterns of system matrices for advection-diffusion 1D singleequation discretizations.

mesh of Figure 6.1(b). As can be seen, both the diagonal and off-diagonal blocks are sparse because of the tensor-product basis expansions, but they are much denser than in the purely advective case. This is a consequence of the additional spatial derivatives. Moreover, it is again impossible to reorder the G-DGSEM matrix of Figure 6.5(a) to obtain a block-diagonal $\underline{\mathbf{A}}_{i i}$ matrix. In contrast, the matrix resulting from the Gauss-Lobatto discretization (Figure 6.5(b)) is suitable for static condensation. This is clearly seen from the appearance of some off-diagonal blocks, like the ones connecting elements 1-3 or 1-5. However, the off-diagonal blocks that connect elements 1-2 or 3-4 seem to have a more complicated sparsity pattern that does not allow to obtain block-diagonal matrices when reordering. This is just an artifice of the plotting. In fact, a detailed view of the part of the Jacobian that corresponds to elements 1 and 2 (Figure 6.6) reveals that only some of the degrees of freedom are coupled in the Gauss-Lobatto DGSEM.

In summary, the off-diagonal blocks of a GL-DGSEM discretization only take nonzero values if

- $j$ and $r$ correspond to boundary degrees of freedom (advective case), or

- $j$ or $r$ correspond to boundary degrees of freedom (diffusive case).

In either case, the system can be reorganized as a Schur complement problem with $\underline{\mathbf{A}}_{i i}$ being a block-diagonal matrix. 


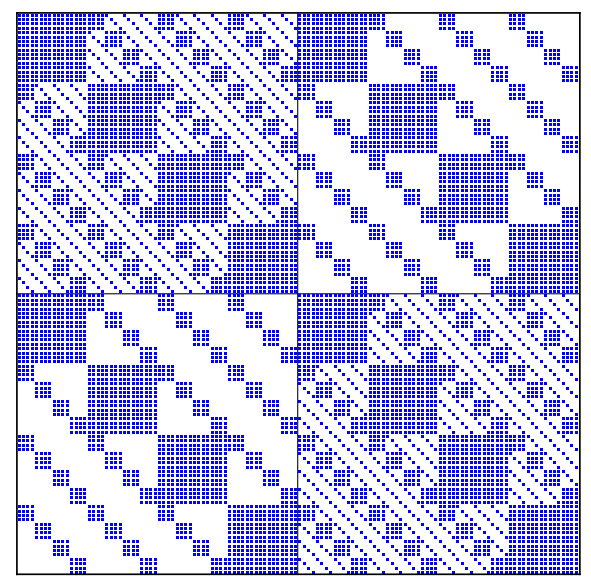

(a) DGSEM on Gauss nodes

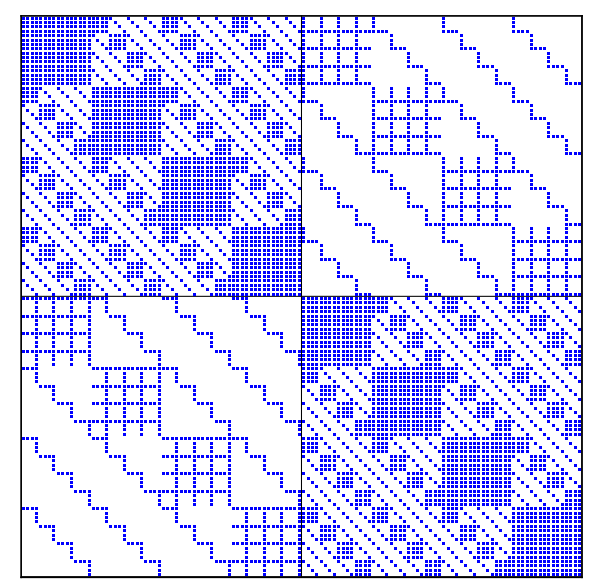

(b) DGSEM on Gauss-Lobatto nodes

Figure 6.6: Detail of the Jacobian blocks corresponding to elements 1 and 2.

\subsection{Static-Condensation for the GL-DGSEM}

As was shown in previous section, the linear system resulting from the GL-DGSEM discretization of an advection, diffusion or advection-diffusion conservation law can be reorganized and condensed to obtain a new system of the form,

$$
\left[\begin{array}{cc}
\underline{\mathbf{A}}_{b b}-\underline{\mathbf{A}}_{i b} \underline{\mathbf{A}}_{i i}^{-1} \underline{\mathbf{A}}_{b i} & \mathbf{0} \\
\underline{\mathbf{A}}_{b i} & \underline{\mathbf{A}}_{i i}
\end{array}\right]\left[\begin{array}{l}
\mathbf{Q}_{b} \\
\mathbf{Q}_{i}
\end{array}\right]=\left[\begin{array}{c}
\mathbf{B}_{b}-\underline{\mathbf{A}}_{i b} \underline{\mathbf{A}}_{i i}^{-1} \mathbf{B}_{i} \\
\mathbf{B}_{i}
\end{array}\right],
$$

where $\underline{\mathbf{A}}_{i i}$ is a block-diagonal matrix. In addition, as was shown in Section 6.2, this new linear system can be solved in two steps: the linear solve of the condensed system and the reconstruction of the solution on the inner degrees of freedom.

The construction of the condensed system is graphically represented in Figure 6.7 for the simple 3D mesh of Figure 6.1(b) and for the compressible Navier-Stokes equations $\left(n_{\text {cons }}=5\right)$.

A few remarks can be made.

- The blocks of matrix $\underline{\mathbf{A}}_{i i}$ keep the tensor-product sparsity and the whole matrix can be inverted locally (element by element).

- The condensed matrix keeps the diagonal dominance with a seemingly denser structure.

- In fact, the condensed system matrix exhibits element connectivities that were not spotted in the global matrices of last section: In spite of the use of a 
compact viscous numerical flux, there are off-diagonal entries that suggest a neighbors of neighbors coupling. This behavior is not observed in the purely advective case (not shown here).
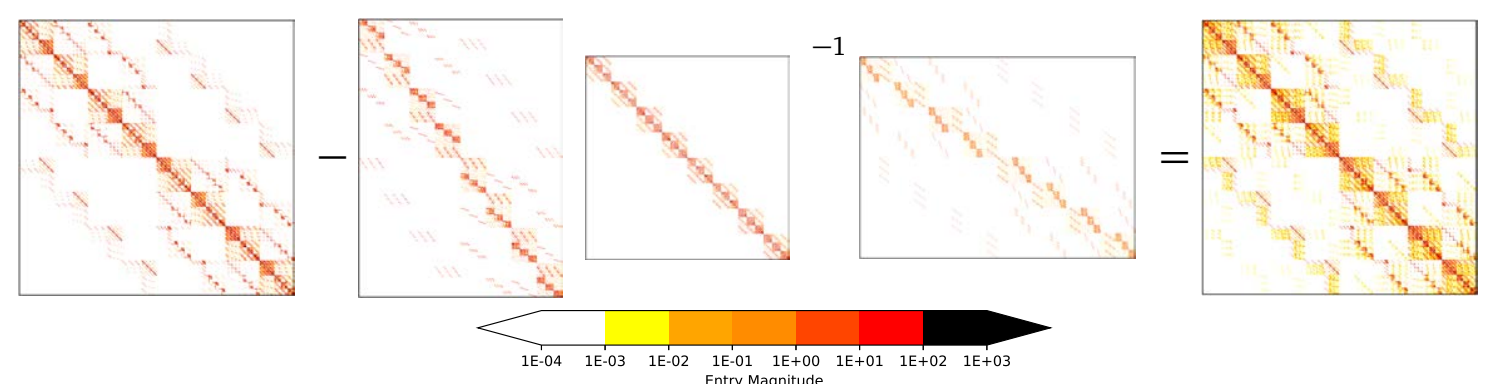

Figure 6.7: Matrix condensation operations for the 3D Navier-Stokes $\left(n_{\text {cons }}=5\right)$ case: $\underline{\mathbf{A}}_{b b}-\underline{\mathbf{A}}_{i b} \underline{\mathbf{A}}_{i i}^{-1} \underline{\mathbf{A}}_{b i}=\underline{\mathbf{A}}_{C}$. Every pixel corresponds to a matrix entry.

The ratio of the condensed system size $\left(n_{1}\right)$ to the global system size $\left(n_{1}+n_{2}\right)$ is a function of the polynomial order. Supposing $p$-isotropic discretizations, the maximum ratio is

$$
\left.\frac{n_{1}}{n_{1}+n_{2}}\right|_{\max }=\frac{(N+1)^{d}-(N-1)^{d}}{(N+1)^{d}},
$$

where $d$ is the number of dimensions of the problem. Note that an advantage in the system size is only observed for $N>1$.

Table 6.1: Ratio of condensed system size to global system size

\begin{tabular}{c|ccc}
$d=$ & 1 & 2 & 3 \\
\hline$\frac{n_{1}}{n_{1}+n_{2}}$ & $\frac{2}{N+1}$ & $\frac{4 N}{(N+1)^{2}}$ & $\frac{6 N^{2}+2}{(N+1)^{3}}$
\end{tabular}

Figure 6.8 shows the ratio of the condensed system size to the global system size as a function of the polynomial order for $p$-isotropic discretizations in every element. The maximum ratio corresponds to the theoretical value that can be computed supposing that all the degrees of freedom on element boundaries contribute to off-diagonal blocks The achievable ratio is problem-dependent (here for the diffuser mesh of Figure 6.1(b)). It is a function of how many element boundaries correspond to domain boundaries. Namely, the degrees of freedom on the domain boundaries only contribute to the diagonal blocks and, therefore, it is not necessary to include them in the $\mathbf{Q}_{b}$ vector. As expected, the static-condensation method provides increasing advantages as the polynomial order is incremented. 


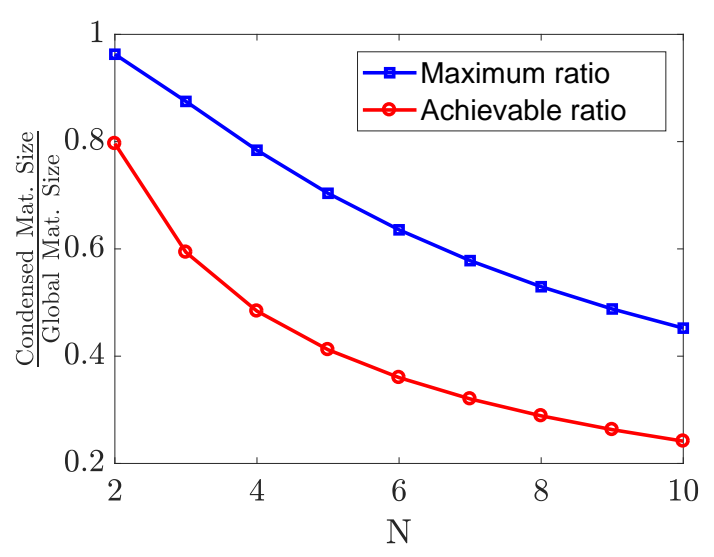

Figure 6.8: Ratio of condensed system size to global system size (3D diffuser example).

Implementation In the following section, a numerical example is presented that was obtained using an implementation of the static-condensation method for the GL-DGSEM. The Jacobian is computed analytically following the derivations of Chapter 5 and stored in four different matrices that correspond to $\underline{\mathbf{A}}_{i i}, \underline{\mathbf{A}}_{b i}, \underline{\mathbf{A}}_{i b}$ and $\underline{\mathbf{A}}_{b b}$. To do that, the mesh connectivities are preprocessed to obtain appropriate permutation indexes for each degree of freedom. The matrices $\underline{\mathbf{A}}_{b i}, \underline{\mathbf{A}}_{i b}$ and $\underline{\mathbf{A}}_{b b}$ are stored in sparse CSR formats and the blocks of $\underline{\mathbf{A}}_{i i}$ are stored as dense matrices. The matrix-matrix multiplications are performed with the routines provided by the BLAS libraries [24] and the individual blocks of $\underline{\mathbf{A}}_{i i}$ are inverted using the LU decomposition routines of the LAPACK [4] library with no regard of the tensor-product properties.

\subsection{Numerical Example}

In this section, we test the computational performance of the statically condensed time-implicit GL-DGSEM and compare it with the traditional (not statically condensed) time-implicit GL-DGSEM and a time-explicit GL-DGSEM. The flow past a cylinder at $\operatorname{Re}_{\infty}=30$ and $\mathrm{Ma}_{\infty}=0.2$ is simulated using polynomial orders that range between $N=3$ and $N=7$. The Lax-Friedrichs flux is used as the advective numerical flux, $\hat{\mathbf{f}}^{a}$, and the symmetric interior penalty method is used as the diffusive numerical flux, $\hat{\mathbf{f}}^{v}$ and $\hat{\mathbf{q}}$. All simulations were run with HORSES3D (Appendix C) in a 24-core Xeon broadwell e5-2690v4 and the Jacobians of the time-implicit simulations were computed analytically. Figure 6.9 shows the horizontal velocity contours and the mesh used for this test case.

The time-explicit simulations use the Willamson's low-storage 3rd order RungeKutta method [203] as the time-marching scheme, where the time step is dynami- 


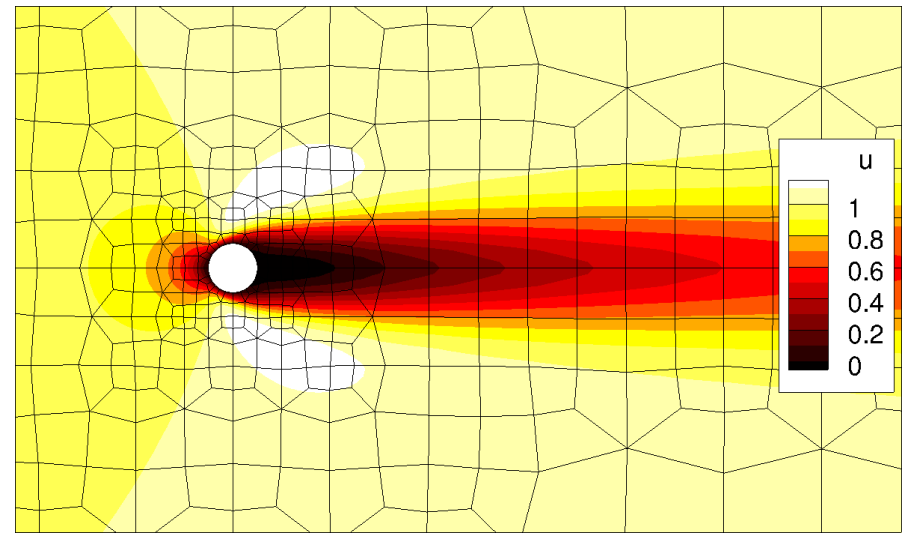

Figure 6.9: Cylinder test case.

cally adapted according to the CFL condition (see Section 3.2.2). The time-implicit simulations use a BDF1 (backward Euler) scheme where the time-step size is fixed to $\Delta t=1$ for simplicity. In each case, the linear system (condensed and not) that results from the Newton linearized BDF1+DGSEM discretization is solved using the implementation of the parallel direct sparse solver (PARDISO) that is present in Intel's Math Kernel Library (MKL).

All simulations are restarted from an $N=3$ approximation after $10^{4}$ explicit RK3 time steps are taken, which is in turn started from a uniform flow condition. The main reason is that that number of explicit time steps is computationally cheap to compute and that, after those $10^{4}$ time steps, the flow conditions are evolved enough to provide Jacobian matrices that can be reused for multiple solves. If the implicit simulations were started from a uniform flow condition, the Jacobian matrix would have to be computed several times at the beginning of the simulation, which may affect performance. All simulations are time-marched until reaching steady-state, which is assumed when the residual is $\|\tilde{\mathfrak{R}}\|_{\infty} \leq 10^{-9}$.

Figure 6.10 shows the evolution of the residual as a function of the elapsed CPU-time for the simulations of order $N=3, N=5$ and $N=7$. Both subfigures illustrate the same data, but Figure 6.10(a) is a semi-log plot, where the start of the simulation can be seen in detail, and 6.10(b) is a log-log plot, which allows one to see the overall performance of the simulation.

As can be seen in Figure 6.10, the convergence rate of the purely explicit simulations is very low, specially at high polynomial orders. In fact, the time-implicit methods outperform them in each case. It is also noteworthy that there is a sudden increase in the residual after the high-order simulations are restarted from the $N=3$ approximation. This increase is the residual that corresponds to the highfrequency errors of the low-order $N=3$ approximation.

In the implicit simulations, there is a plateau after the sudden residual increase, which mainly corresponds to the Jacobian factorization times. The Jacobian com- 


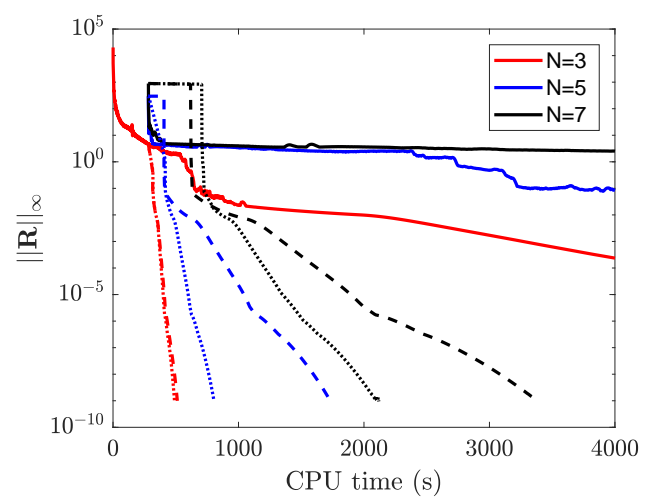

(a) Semi-log plot of the residual evolution

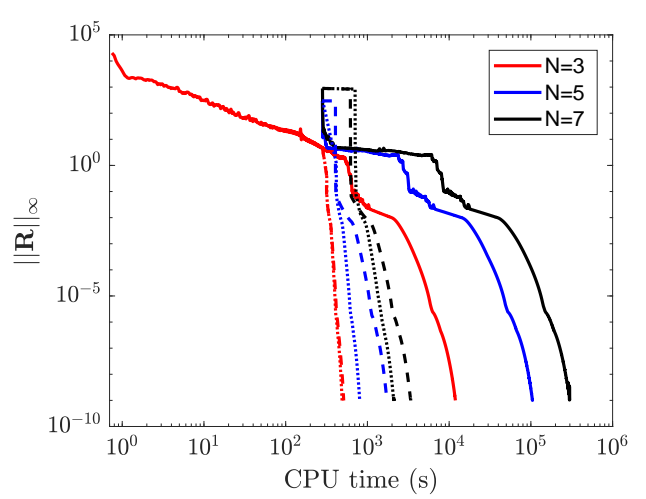

(b) Log-log plot of the residual evolution

Figure 6.10: Residual norm vs. CPU-time for the cylinder flow at $\operatorname{Re}_{\infty}=30$. Solid lines represent the purely explicit simulations (RK3), dashed lines are the implicit simulations solved globally (BDF1), and dotted lines are the implicit simulations solved with the static-condensation method (BDF1+static-condensation).

putation time also adds to the plateau, but it is negligible with respect to the computational cost of the LU decomposition.

There is a small overhead in the statically condensed simulations with respect to the global ones, as can be easily appreciated in the duration of the plateau for the simulations of $N=7$. This extra computing time is invested in assembling the condensed system, which requires inverting the blocks of $\underline{\mathbf{A}}_{i i}$, performing matrix-matrix multiplications and subtractions for the condensed matrix, and matrix-vector multiplications for the condensed RHS.

The statically condensed simulations are computationally more efficient than their globally solved counterparts. Namely, the extra computational resources that are needed for the condensation operations are rewarded with a faster convergence rate. In other words, the slope of the decrease of the residual norm is steeper for the statically condensed simulations.

Figure 6.11 shows the performance of the statically condensed and globally solved simulations with respect to the explicit simulations. It can be seen that significant speed-ups can be achieved with both methods, but that the statically condensed excel when the polynomial order is increased. The speed-up is as high as 140 for $N=7$. The change of slope that is observed in the statically condensed speed-ups for high polynomial orders is likely to be caused by the extra operations of the static-condensation method, which in this work are performed with the global sparse matrices. In any case, the convergence rate of the simulations where PARDISO is applied to the statically condensed system, is always higher than the one of simulations where PARDISO is applied to the global system.

A further advantage of using static condensation is that the statically condensed 


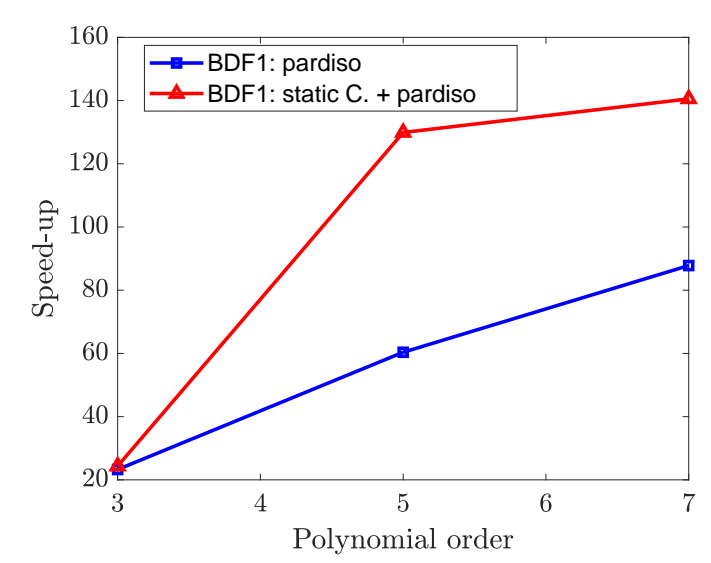

Figure 6.11: Enhanced performance of the static-condensation method for the cylinder test case.

system, besides being smaller in size, is better conditioned than the original global system. This behavior was observed by Sherwin et al [188] for their statically condensable DG method, and is shown in Figure 6.12 for the system matrices that come from the time-implicit GL-DGSEM discretization of the flow past a cylinder at $\operatorname{Re}_{\infty}=30$ and $\mathrm{Ma}_{\infty}=0.2$.

The $L_{2}$ condition number of the Jacobian matrices was determined as the ratio of the largest and smallest eigenvalues, which were estimated using the shift-andinvert algorithm for sparse matrices that is implemented in the ARPACK library [122]. In the example presented here, the maximum eigenvalue of the global and the statically condensed systems are very close. The condition number of the latter is smaller, mainly because the minimum eigenvalue is moved to the left (away from the complex plane origin).

The globally solved time-implicit discretizations suffer from an even-odd behavior, which is the reason why no results are reported for even polynomial orders in Figures 6.10 - 6.12. The PARDISO solver is not able to converge on the global system for the discretizations of order $N=4$ and $N=6$ with the specified settings: $\Delta t=1$, when the simulations are restarted from the $N=3$ solution after $10^{4}$ time steps.

The even-odd behavior is not observed in the statically condensed system, which can be solved with PARDISO using the exact setup as for odd $N$. In fact, the computation times for even polynomial orders fall between the ones obtained for odd polynomial orders, as one would expect. This is remarkable since it means that static condensation provides robustness to the linear problem of the analyzed case and allows to obtain adequate solutions in otherwise unsolvable cases.

Several strategies were tested to obtain the solution of the non-convergent (globally solved) cases: 


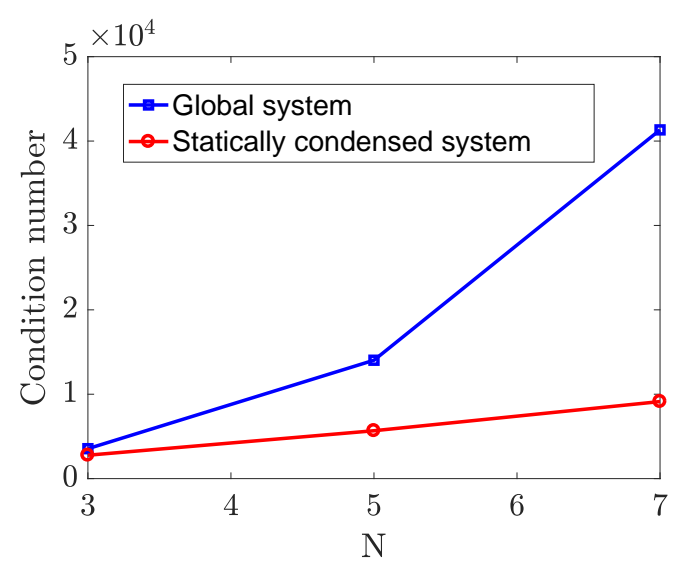

Figure 6.12: L2 condition number of the system matrix for the global system and the statically condensed one.

1. Modify the PARDISO parameters, such as the number of iterative refinement steps and the pivot perturbation. This yielded no improvement.

2. Restart from a solution of order $N=4$ after $10^{4}$ time steps (instead of order $N=3$ ). This did not work for any of the problematic cases.

3. Restart from a solution of order $N=4$ after $2 \times 10^{4}$ time steps. This only worked for the $N=6$ test case.

4. Restart from a solution of order $N=4$ after $2 \times 10^{4}$ time steps and also reduce the time-step size to $\Delta t=0.1$. This worked for both $N=4$ and $N=6$.

Figure 6.13 shows the evolution of the residual as a function of the CPU-time for the even polynomial orders, $N=4$ and $N=6$. Strategy 4 is retained for $N=4$ and Strategy 3 is retained for $N=6$, since that one provides a better performance than Strategy 4. As can be seen, the advantages of using the static-condensation method are more evident for even polynomial orders in this particular test case.

The lower condition number of the statically condensed linear systems suggests that the static-condensation method may also improve the convergence rate when using Krylov subspace linear solvers. In fact, the convergence rate of a Krylov subspace method is directly related to the spectrum of the linear operator [182]. We now present a test that supports that idea.

In this test, the linear system is solved using a GMRES solver (see Section 4.3.3.1 for a description of the GMRES method) with a simple point Jacobi preconditioner. Specifically, the GMRES solver that is implemented in the PETSc library [1] is used. Figure 6.14 shows the linear system residual as a function of the GMRES iterations for polynomial orders $N=3$ and $N=5$. When a time step starts to be solved, a large increase in the linear system residual is exhibited, and every time a new Newton iteration starts there is a small increase in the linear system residual. 


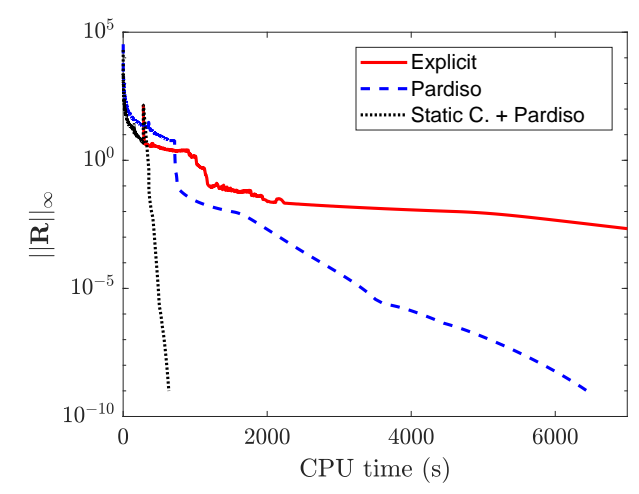

(a) $N=4$

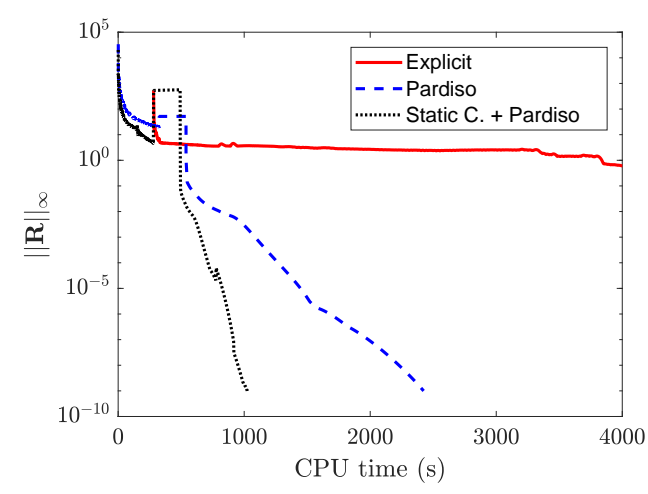

(b) $N=6$

Figure 6.13: Residual norm vs CPU-time for the cylinder at $\operatorname{Re}_{\infty}=30$. Even polynomial orders.

As can be seen in Figure 6.14(a), the $N=3$ statically condensed simulation takes several time steps while the globally solved one fails to converge the Newton method and reaches the maximum number of iterations allowed before starting the next time step. This behavior is even more pronounced in the $N=5$ simulation, where the statically condensed simulation advances while the globally solved one diverges.

All in all, although a very simple preconditioner is used (The point Jacobi preconditioner is known to be sub-optimal for high-order methods $[157,173]$ ), the test shows that statically condensing the GL-DGSEM has a positive impact in the convergence rate when using GMRES for the selected test case.

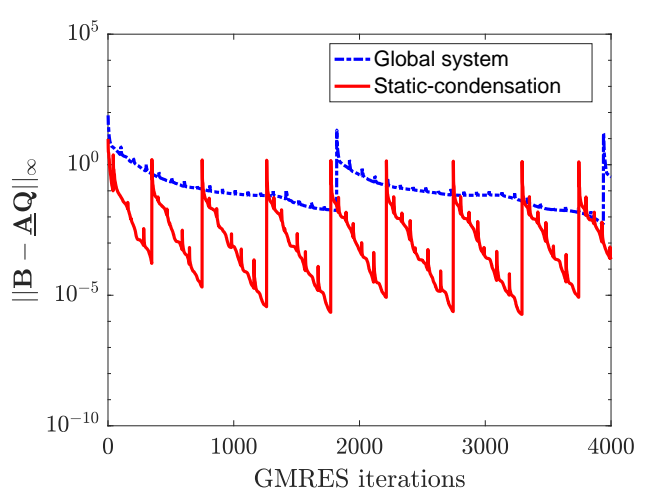

(a) $N=3$

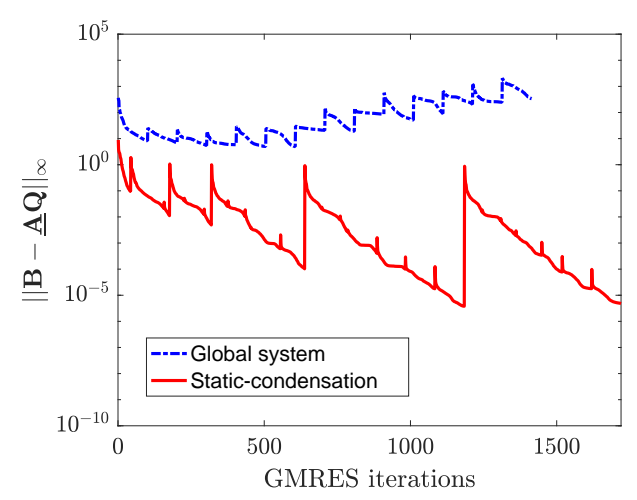

(b) $N=5$

Figure 6.14: Performance of the static-condensation method with a GMRES solver. 


\subsection{Final Remarks}

In this chapter, we have shown that the DGSEM with Gauss-Lobatto nodes can be directly formulated as a Schur complement problem and solved in an efficient manner using static condensation, where the matrix that needs to be inverted to obtain the Schur complement is block-diagonal. As a result, a static-condensation method for the time-implicit GL-DGSEM is presented, which does not impose strong constraints on the choice of the basis functions or the form of the numerical fluxes (as other static-condensation DG methods).

It is shown, by means of a numerical example with the compressible NavierStokes equations, that static condensation produces a speed-ups of up to 140 when compared to the time-explicit GL-DGSEM, and a speed-up of up to 2 when compared with the time-implicit GL-DGSEM that solves the global system directly.

We have shown that the method proposed here does not suffer from a detrimental even-odd behavior, which affects the globally solved system, when solving the subsonic flow past a cylinder. As a result, we can conclude that statically condensing the system provides robustness to the implicit time-discretization of the GL-DGSEM.

In addition, the statically condensed matrices of the presented example are better conditioned than the global matrices they are constructed from, since the minimum eigenvalue is moved away from the origin of the complex plane. This is a likely reason of the improved robustness and may allow one to use certain timeintegration schemes that are otherwise unstable (such as BDF3 or higher, which are not A-stable).

The findings in this chapter constitute a further advantage of using GL-DGSEM over G-DGSEM or other variants of the DG method. In summary, GL-DGSEM is computationally cheaper, easier to implement, it enables the formulation of provably stable uncrashable schemes, allows larger time steps in time-explicit discretizations, enables unified formulations of certain viscous numerical fluxes, and can be used to formulate a statically condensed DG method.

As a final remark, the static-condensation operations may be performed locally (instead as sparse matrix operations), as in HDG methods, but additional research is needed to obtain a consistent local formulation. This may reduce the storage requirements and improve the condensed system's construction, which in turn could increase the observed speed-ups. Moreover, the study of appropriate preconditioners for Schur complement problems and methods based on tensor-product bases is still an active area of research that could contribute to the further development statically condensed DGSEM methods. Interesting alternatives for Schur complement preconditioners have been developed by Gatto et al. [81], and for tensor-product preconditioners by Pazner and Persson [154]. 



\section{Part III \\ Enhancing the Spatial Discretization: p-Adaptation}

Summary In Part II, we reviewed and developed several strategies that improve the computational efficiency of high-order DG discretizations. Those strategies used a fixed spatial discretization and speed-ups were obtained by using techniques like multigrid, implicit time-integration schemes and static condensation. Another possibility to reduce the computational time is to use local mesh adaptation strategies, which reduce the number of degrees of freedom (i.e. change the spatial discretization) in a smart way, so that the accuracy of the method is not deteriorated.

This part is devoted to the development and implementation of efficient mesh adaptation strategies for steady and unsteady flows. Specifically, we will be dealing with anisotropic $p$-adaptation methods for discontinuous Galerkin methods.

In Chapter 7, an overview of the different concepts needed to perform $p$-adaptation in DG methods is presented: mortar spaces for $p$-nonconforming discretizations, definition of error measures and the $\tau$-estimation method. In Chapter 8 , a novel, accurate and low-cost truncation error estimator for the $p$-anisotropic DGSEM is presented and validated. In Chapter 9, this truncation error estimator is used to develop a $p$-adaptation method for steady flow problems. Finally, Chapter 10 provides different strategies to perform $p$-adaptation for unsteady flow problems using anisotropic truncation error estimates. 



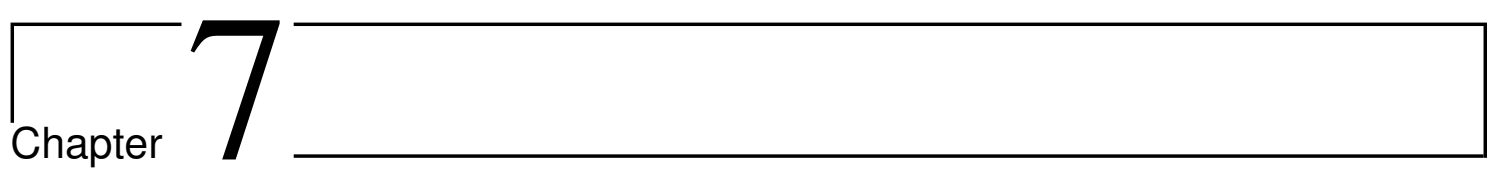

\section{Background: On $p$-Adaptation and Error Estimation}

\section{Contents}

7.1 Introduction $\ldots \ldots \ldots \ldots \ldots \ldots \ldots \ldots \ldots \ldots$

7.2 Background on Error Estimation and $p$-Adaptation $\ldots \ldots . .98$

7.3 Definition of Errors . . . . . . . . . . . . . . . . 102

7.3.1 Notation . . . . . . . . . . . . . . . . . . . . . . . . 102

7.3 .2 Interpolation Error . . . . . . . . . . . . . . . . . 102

7.3.3 Quadrature Error . . . . . . . . . . . . . . . . . . 103

7.3.4 Discretization Error . . . . . . . . . . . . . . . . . . . 103

7.3.5 Truncation Error . . . . . . . . . . . . . . . . . . . 105

$7.4 \tau$-Estimation Method . . . . . . . . . . . . . . 108

7.5 Nonconforming Discretizations and Mortar Spaces . . . . . 110

\subsection{Introduction}

As explained in Chapter 1, local adaptation can be performed by enriching or reducing the polynomial order in certain elements ( $p$-adaptation), by subdividing or merging elements ( $h$-adaptation), or by relocating the position of the nodes in a mesh ( $r$-adaptation). $p$-Adaptation is natural to high-order methods where the approximation degree can be modified easily, and is especially useful when the solution is smooth, since in that case the error decreases exponentially with the polynomial order $[64,176,177]$. 
When shocks or other kind of non-smooth features are present in the solution inside an element, increasing the polynomial order may lead to spurious oscillations in the solution and instabilities in the solver [37, 200]. In such cases, the use of $h$ - or $r$-adaptation methods is preferred since they decrease the element size and, consequently, may cause the non-smoothness to move to an element interface (where the DG method can describe it appropriately), or at least they may contribute to the solution being smoother inside the element.

In this thesis, we deal only with $p$-adaptation methods since they exploit the high-order character of DG schemes. However, the error estimators and adaptation strategies that are presented in following chapters can be extended to $h$ - and $r$ adaptation methods.

In the DGSEM, and any other DG method with a tensor-product basis, one can take advantage of the fact that the polynomial order in each reference coordinate direction can be defined independently (see Section 2). In fact, it was shown by Kompenhans et al. [111] that performing anisotropic $p$-adaptation is more efficient than isotropic $p$-adaptation, since the same accuracy can be reached with a fewer number of degrees of freedom. For that reason, the $p$-adaptation strategies that are presented in this thesis exploit the $p$-anisotropy of the DGSEM.

To perform local anisotropic $p$-adaptation, three key tools are needed: a local measure of the error, an algorithm that changes the polynomial orders anisotropically according to the error measure, and a way to couple elements that have different polynomial orders (i.e. that are $p$-nonconforming).

This chapter is organized as follows. Section 7.2 contains a general background on error estimation and $p$-adaptation, and provides the reasons why the truncation error is used in this thesis to drive anisotropic $p$-adaptation methods. Section 7.3 contains several error definitions that will be used in the following chapters and presents insight into the convergence properties of the different error measures. Thereafter, Section 7.4 details the $\tau$-estimation method, the fundamental method that is used to estimate the truncation error in the following chapters. Finally, in Section 7.5, the mortar element method is described, which allows to couple $p$ nonconforming elements when using the DGSEM.

\subsection{Background on Error Estimation and p-Adaptation}

The error of a numerical approximation is a quantitative measure of how accurately a numerical solution satisfies the governing mathematical equations [153, $169,174]$. The error is generally considered as the difference between the exact solution and the discrete one. Since the exact solution is generally unknown, it is necessary to estimate the error. A precise assessment of the numerical errors is useful for defect correction [140, 169] and for mesh adaptation strategies $[141,142,208]$. 
Defect correction is a technique that enables high accuracy by correcting the numerical solution using an estimate of the error [140, 169]. It requires highly accurate estimates of the discretization error and, therefore, a significant amount of computational resources is usually invested in the error estimation procedure [159].

In the case of mesh adaptation strategies, accuracy is important but it is not everything, as the computational cost of the error estimation also plays an important role. In fact, even unsophisticated error measures (e.g. the gradients of the solution $[2,156]$ ) can be useful to improve computational efficiency.

Historically, adaptatation strategies have been classified according to the type of error measure that is employed as feature-based adaptation, adjoint-based adaptation, and local error-based adaptation. A comparison of these three approaches was performed by Fraysse et al. [73] for finite volume approximations and by Kompenhans et al. [111] and Naddei et al. [150] for high-order DG methods. The key ideas behind the adaptation approaches are:

- The feature-based adaptation is the classical approach and uses easy-to-compute error measures that depend on the flow features. They rely on the assumption that high errors are expected where the flow is more difficult to resolve. Hence, refinement is predicted where high velocity, density or pressure gradients are identified [2, 156]. For DG discretizations, an easy-to-compute feature-based adaptation criterion is the assessment of jumps across element interfaces [119, 120, 167].

The main disadvantage of these methods is that there is no direct relation between the adaptation criterion and the numerical errors and thus the accuracy is not easily predictable. Additionally, the only way to solve steady-state problems is to adapt iteratively.

- A second and more sophisticated approach is known as adjoint-based adaptation. In this approach, a functional target is defined (e.g. drag or lift in external flow aerodynamics) and the adjoint problem is solved to obtain a spatial distribution of the functional error, which is then used to adapt the mesh. This technique was originally developed for structural analysis using FEM by Babuška and Miller $[10,11]$. Later, it was applied to CFD applications using FEM by Johnson and Rannacher [103] and Becker and Rannacher [18]. Giles and Pierce first used it for Finite Volume schemes [84, 160, 161, 196], and more recently it has been used for adaptation strategies in DG methods [92, 93, 200].

The main drawback of this approach is the high computational cost to solve the adjoint problem and the storage requirements needed to save the error estimators, especially in unsteady flows. Moreover, only the error of the functional analyzed is guaranteed to be reduced, whereas the error of other functionals may deteriorate. 
- A computationally more efficient alternative is the local error-based adaptation, which is based on the assessment of any measurable (not feature-based) local error in all the cells of the domain [44, 93, 121, 141, 142]. The local error-based adaptation methods are interesting since, in contrast to featurebased methods, they provide a way to predict and control the overall accuracy, and are computationally cheaper than adjoint-based schemes [110, 111].

For those reasons, local error-based adaptation strategies are retained in this work and will be the topic of research in what remains of this thesis.

A large amount of effort has been invested in the development of reliable local error-based adaptation methods. Estimations of the local discretization error $\left(\mathbf{q}-\mathbf{q}^{N}\right)$ have been used by Mavriplis $[141,142]$ to develop $h p$-adaptation techniques for the spectral element method. Later, Casoni et al. [44] extended that approach to adapt the artificial viscosity in shock capturing discontinuous Galerkin discretizations.

Residual-based $p$-adaptation is also a local error-based adaptation method, which uses the residual to measure how accurate is the local approximation. This method was originally developed for Finite Elements (FE) and has been successfully used with DG methods [93, 150].

In the case of modal (hierarchical) DG methods, a possibility is to employ low cost error estimates that take advantage of the modal approximation to drive $p$ adaptation procedures, such as the Variational Multiscale (VMS) indicator by Kuru and De la Llave Plata [121], or the spectral decay indicator by Persson and Peraire [156]. These have in common that they measure the relative contribution of the different modes to a quantity (kinetic energy, enthalpy, entropy, etc.). If the quantity is concentrated in the highest-order modes, refinement is performed. This resembles feature-based adaptation methods since energetic high-order modes can be found when large gradients in the solution are present. To obtain an adapted mesh with these methods, multiple error estimation and $p$-adaptation stages must be performed. Each time, a number of elements of the domain are marked for refinement or coarsening depending on an error threshold and then adapted. This procedure is done iteratively until an acceptable approximation is found.

In this thesis, we favor truncation error estimators, another local error-based alternative to drive a mesh adaptation method [19, 72, 73, 74, 191, 192]. The truncation error is related to the discretization error through the Discretization Error Transport Equation [174], where it acts as a local source term (see Sections 7.3.4 and 7.3.5). This relation makes it useful as an indicator for mesh adaptation methods $[46,191]$, since refining the mesh where the truncation error is high reduces the discretization error in all the mesh [176], with an additional advantage: truncation error estimation requires less computational effort than adjoint methods.

In hyperbolic problems, the discretization error is strongly advected, i.e. it is transmitted downstream from under-resolution areas, but the truncation error is 
only weakly advected [176]. Therefore, an adaptation procedure that uses the truncation error specifically targets the under-resolved areas, whereas one based on the discretization error targets the under-resolved areas and the zones downstream of them $[176,177]$, which makes the truncation error more suitable for adaptation purposes than the discretization error. Finally, it has been shown that controlling the truncation error targets the numerical accuracy of all functionals at once [110], ensuring that adapting a mesh using the truncation error leads necessarily to an error decrease in any other functional (e.g. lift or drag).

The $\tau$-estimation method proposed by Brandt [33], which estimates the local truncation error by injecting a fine grid solution into coarser meshes, has been used to perform local error-based mesh adaptation in low-order schemes [19, 72, 73, 74, $191,192]$. Rubio et al. [175] extended the $\tau$-estimation approach to high-order methods using a continuous Chebyshev collocation method. Later, Rubio et al. [176] applied it to DGSEM discretizations, and studied the quasi-a priori truncation error estimation, which allows one to estimate the truncation error without having fully converged fine solutions. Kompenhans et al. [110] applied the $\tau$-estimation approach to perform steady-state $p$-adaptation using the Euler and Navier-Stokes equations, and showed that a reduction of the truncation error increases the numerical accuracy of all functionals at once. Furthermore, Kompenhans et al. [111] also showed that $\tau$-based adaptation can exhibit better performance than feature-based adaptation.

In contrast to most local error-based adaptation methods, where multiple error estimation and adaptation stages are needed in a steady-state solution, the $\tau$-estimation method generates a unique prediction of what polynomial order is needed for a desired truncation error threshold. Therefore, in steady-state the adaptation strategy is to converge a high-order approximation (reference mesh) to a specified global residual and then to perform a single error estimation followed by a corresponding $p$-adaptation process.

The truncation error estimator bears a strong resemblance to residual-based indicators, since its formulation departs from the residual equation (as shown in Section 7.3.5). Moreover, it is also similar to error estimates based on hierarchical bases because the $\tau$-estimation measures the error by comparing approximations of different polynomial orders. As the Fourier coefficients of an approximation, the truncation error is known to decay spectrally in smooth solutions $[110,176,177]$. Therefore, if the estimation is good, it is possible to extrapolate the behavior and predict the polynomial order needed for a desired error threshold [110, 180].

Being a relatively recent technique, $p$-adaptation methods that use $\tau$-estimators have only been applied to steady-state solutions with high-order methods [110, 111]. Unsteady $p$-adaptation using truncation error estimates will be addressed in Chapter 10. 


\subsection{Definition of Errors}

In this section, we define some of the error measures that will be used in the following chapters to derive efficient error estimators and to drive anisotropic $p$ adaptation methods.

\subsubsection{Notation}

The error measures used in this thesis have a specific notation, and different super and subscripts that give information about the quantity that is represented.

We generally refer to the error of a variable that is a state vector, such as the numerical solution of order $N, \mathbf{q}^{N}$. Therefore, the error measures are commonly noted with bold lowercase letters: $\varepsilon^{N}$ for the interpolation error, $\epsilon^{N}$ for the discretization error, $\mathrm{e}^{N}$ for the quadrature error, or $\tau^{N}$ for the truncation error. These variables can be arranged into generic vectors (bold uppercase letters) when we consider the error on several discretization nodes, e.g.

$$
\begin{aligned}
\mathscr{T}^{N} & =\left[\tau_{1}^{N}, \tau_{2}^{N}, \tau_{3}^{N}, \tau_{4}^{N}, \ldots\right]^{T}, \\
\mathscr{E}^{N} & =\left[\epsilon_{1}^{N}, \epsilon_{2}^{N}, \epsilon_{3}^{N}, \epsilon_{4}^{N}, \ldots\right]^{T},
\end{aligned}
$$

where subscripts refer to discretization node numbers.

We will distinguish between locally-generated and externally-generated truncation and discretization errors. The locally-generated errors are noted with as $\tau_{\Omega^{e}}^{N}$ or $\epsilon_{\Omega^{e}}^{N}$, and the externally-generated errors are noted as $\tau_{\partial \Omega^{e}}^{N}$ or $\epsilon_{\partial \Omega^{e}}^{N}$.

\subsubsection{Interpolation Error}

Definition 7.1 (Interpolation error) The difference between a function and its polynomial interpolant of order $N$ is the interpolation error,

$$
\boldsymbol{\varepsilon}_{\mathbf{q}}^{N}=\mathbf{q}-\mathbf{I}^{N}(\mathbf{q})
$$

where $\mathbf{I}^{N}(\mathbf{q})$ is the projection of the function $\mathbf{q}$ into the polynomial space of order $N$. The interpolant, $\mathbf{I}^{N}(\mathbf{q})$, is one of the few functions that is noted as an uppercase letter although it returns the same type of vector as its argument. The reason is that it is closely related to interpolation operators.

For sufficiently smooth functions, in the asymptotic range, the interpolation error in an element $e$ behaves as $[176,177]$

$$
\left\|\left.\varepsilon_{\mathbf{q}}^{N}\right|_{\Omega^{e}}\right\|_{\infty} \leq C_{0}^{e} e^{-N^{e} \eta_{0}^{e}}
$$

where $C_{0}^{e}$ and $\eta_{0}^{e}$ are constants that depend on the local smoothness of the function q $[42,95]$ and $N^{e}$ is the local polynomial order in the element $e$. In the DGSEM, the use of tensor-product bases in $d$ dimensions allows decoupling the interpolation 
error in directional components, each of which depends solely on the polynomial order in the corresponding direction,

$$
\boldsymbol{\varepsilon}_{\mathbf{q}}^{N}=\sum_{i=1}^{d} \boldsymbol{\varepsilon}_{\mathbf{q}, i}^{N} \text { such that } \boldsymbol{\varepsilon}_{i}=\boldsymbol{\varepsilon}_{i}\left(N_{i}\right) .
$$

As a consequence, in the $p$-anisotropic DGSEM, the interpolation error exhibits a tensor-product-type error bound in $d$ dimensions inside every element [176],

$$
\left\|\left.\boldsymbol{\varepsilon}_{\mathbf{q}}^{N}\right|_{\Omega^{e}}\right\|_{\infty} \leq \sum_{i=1}^{d} C_{0, i}^{e} e^{-N_{i}^{e} \eta_{0, i}^{e}}
$$

\subsubsection{Quadrature Error}

Definition 7.2 (Quadrature error) The quadrature error, also referred to as the numerical integration error, is the difference between the exact integral of a function and its approximation by a Gaussian quadrature:

$$
\mathbf{e}_{\Omega^{e}}^{N}=\int_{\Omega^{e}} \mathbf{q} \mathrm{d} \Omega-\int_{\Omega^{e}}^{N} \mathbf{q} \mathrm{d} \Omega,
$$

where the superindex $N$ on the integral indicates that it is approximated using a Gaussian quadrature of order $N$,

$$
\int_{\Omega^{e}}^{N} \mathbf{q} \mathrm{d} \Omega=\sum_{j=1}^{\mathrm{NDOF}^{e}} J_{j} \mathbf{q}_{j} w_{j} .
$$

In (7.8), $\mathrm{NDOF}^{e}$ is the number of degrees of freedom in element $e(2.6), J_{j}$ are the determinant of the Jacobians of the transformation, $\Omega \rightarrow[-1,1]^{d}$, and $w_{j}$ are the quadrature weights.

\subsubsection{Discretization Error}

Definition 7.3 (Discretization error) The difference between the exact solution to the problem, $\mathbf{q}$, and the one obtained with a discretization of order $N, \mathbf{q}^{N}$, is the discretization error,

$$
\boldsymbol{\epsilon}^{N}=\mathbf{q}-\mathbf{q}^{N}
$$

The discretization error is perhaps the most relevant error measure, since the main idea of any mesh adaptation method is to reduce the difference between the exact and discrete solutions. However, it is also the most difficult error to estimate [159]. 
To obtain the discretization error, let us consider the discretized PDE expressed point-wise on the discretization nodes,

$$
\underline{\mathbf{M}} \frac{\mathrm{d} \mathbf{Q}^{N}}{\mathrm{~d} t}+\mathfrak{R}^{N}\left(\mathbf{Q}^{N}\right)=\mathbf{0}
$$

where $\mathfrak{R}^{N}$ is called the discrete partial differential operator in sampled form. Note that (7.10) was already obtained in (2.13), although it was written in a slightly different manner. For the moment, we only consider steady-state problems ${ }^{1}$, where the first term of $(7.10)$ vanishes, so that it reduces to

$$
\mathfrak{R}^{N}\left(\mathbf{Q}^{N}\right)=\mathbf{0} .
$$

A sampled version of the continuous PDE in steady-state yields

$$
\mathfrak{R}(\mathbf{Q})=\mathbf{0} \text {, }
$$

where $\mathbf{Q}=I^{N} \mathbf{q}$ is the sampled exact solution, $\boldsymbol{I}^{N}$ is therefore a sampling operator, and $\mathfrak{R}$ is called the continuous partial differential operator in sampled form.

Injecting the exact solution into (7.11) one gets

$$
\mathfrak{R}^{N}(\mathbf{Q})=\mathscr{T}^{N},
$$

where $\mathscr{T}^{N}$ is an extra residual term that in this thesis is called the non-isolated truncation error. Subtracting (7.11) from (7.13), the Discretization Error Transport Equation (DETE) [148] is obtained,

$$
\mathfrak{R}^{N}(\mathbf{Q})-\mathfrak{R}^{N}\left(\mathbf{Q}^{N}\right)=\mathscr{T}^{N} .
$$

The DETE is an auxiliary PDE that can be used to study the behavior of the discretization error. If one wants to have an accurate estimation of the discretization error, one can solve (7.14), but it requires significant computational resources. For a linear partial differential operator, the DETE can be rewritten as

$$
\mathfrak{R}^{N}\left(\mathscr{E}^{N}\right)=\mathscr{T}^{N},
$$

where it is evident that the discretization error is a quantity that is advected and diffused according to $\mathfrak{R}^{N}$, the partial differential operator, and whose source (production) term is the non-isolated truncation error.

Since we want to reduce the error of the approximation, we are interested in knowing how the discretization error behaves as a function of the mesh size and the polynomial order. It has been shown theoretically [95, 115] and experimentally $[76,96]$ that the DGSEM, like other high-order DG methods, has a convergence

\footnotetext{
${ }^{1}$ See Chapter 10 for an extension of all these concepts to unsteady problems.
} 
of order $\mathscr{O}\left(h^{N+1}\right)$. This means that, for sufficiently smooth solutions, a DGSEM discretization of uniform order $N$ in a mesh of uniform size $h$ yields a discretization error that behaves as

$$
\left\|\epsilon^{N}\right\|_{\infty} \leq A h^{N+1}
$$

where $A$ is a constant that depends on the smoothness of the solution.

This expression can be rewritten in terms of an exponential function as

$$
\left\|\epsilon^{N}\right\|_{\infty} \leq C_{\epsilon} e^{-N \eta_{\epsilon}}
$$

where $C_{\epsilon}$ and $\eta_{\epsilon}$ are again constants that depend on the smoothness of the solution, and they include some information about the element sizes. Note that this expression is more general as it holds for meshes with non-uniform $h$.

At the element level, the discretization error is influenced by other elements because of the advection and diffusion properties of the PDE, as seen in (7.14) and (7.15). In fact, we can decouple the discretization error into locally-generated and externally-generated contributions for every element $[110,176]$,

$$
\left.\epsilon^{N}\right|_{\Omega^{e}}=\epsilon_{\Omega^{e}}^{N}+\epsilon_{\partial \Omega^{e}}^{N} .
$$

Therefore, in the $p$-isotropic DGSEM it can be assumed that the discretization error in each element behaves as [176, 177]

$$
\left\|\left.\epsilon^{N}\right|_{\Omega^{e}}\right\|_{\infty} \leq C_{\epsilon}^{e} e^{-N^{e} \eta_{\boldsymbol{\epsilon}}^{e}}+\sum_{\substack{k=1 \\ k \neq e}}^{K} C_{\boldsymbol{\epsilon}}^{* k} e^{-N^{k} \eta_{\boldsymbol{\epsilon}}^{* k}},
$$

where $K$ is the number of elements, $C_{\epsilon}^{e}$ and $\eta_{\epsilon}^{e}$ are constants that depend on the smoothness of the solution in the element $e[42,95]$, and $C_{\epsilon}^{* k}$ and $\eta_{\epsilon}^{* k}$ are constants that depend both on the smoothness of the solution and the advection/diffusion properties of the PDE.

The first term on the right-hand side of (7.19) corresponds to the bound of the locally-generated discretization error $\left(\epsilon_{\Omega}^{N}\right)$, whereas the second term is the bound of the externally-generated discretization error $\left(\epsilon_{\partial \Omega}^{N}\right)$, which gathers the errors that are introduced through the interface.

Note that $\boldsymbol{\varepsilon}^{N}$ is the lower bound of $\boldsymbol{\epsilon}^{N}$. For a $p$-anisotropic discretization in $d$ dimensions, the expression (7.19) becomes [83, 176, 177]

$$
\left\|\left.\boldsymbol{\epsilon}^{N}\right|_{\Omega^{e}}\right\|_{\infty} \leq \sum_{i=1}^{d} C_{\boldsymbol{\epsilon}, i}^{e} e^{-N_{i}^{e} \eta_{\boldsymbol{\epsilon}, i}^{e}}+\sum_{\substack{k=1 \\ k \neq e}}^{K} \sum_{i=1}^{d} C_{\boldsymbol{\epsilon}, i}^{k} e^{-N_{i}^{k} \eta_{\boldsymbol{\epsilon}, i}^{k}}
$$

\subsubsection{Truncation Error}

Performing local refinement in regions with high discretization error improves the overall discrete solution. However, from the analysis in Section 7.3.4, it can be 
inferred that the overall error can be reduced in the same amount if the refinement is done only in the regions where the discretization error is produced, i.e. where the truncation error is high, with an additional advantage: even fewer degrees of freedom are needed. This observation was made and validated by Rubio et al. $[176,177]$.

Two types of truncation errors, the isolated truncation error and the non-isolated truncation error, are defined in this section.

Definition 7.4 (Non-isolated truncation error) The difference between the discrete partial differential operator of order $N$ and the exact partial differential operator applied to the exact solution is the non-isolated truncation error,

$$
\tau^{N}=\mathfrak{r}^{N}\left(\mathbf{I}^{N}(\mathbf{q})\right)-\mathfrak{r}(\mathbf{q})
$$

The common definition of the discrete partial differential operator [110, 111, 176, 177] takes into account the variational formulation (now considering the source term), so that its point-wise values are

$$
\mathfrak{r}_{j}^{N}\left(\mathbf{I}^{N}(\mathbf{q})\right)=\int_{\partial \Omega^{e}}^{N} \hat{\mathbf{f}}\left(\mathbf{I}^{N}(\mathbf{q}), \mathbf{I}^{N}(\mathbf{q}), \vec{n}\right) \phi_{j} \mathrm{~d} S-\int_{\Omega^{e}}^{N} \overleftrightarrow{\mathbf{f}}\left(\mathbf{I}^{N}(\mathbf{q})\right) \cdot \vec{\nabla} \phi_{j} \mathrm{~d} \Omega-\int_{\Omega^{e}}^{N} \mathbf{s}^{N} \phi_{j} \mathrm{~d} \Omega
$$

To maintain consistency, the continuous partial differential operator must be defined in integral form. Traditionally, it is taken as

$$
\mathfrak{r}_{j}(\mathbf{q})=\int_{\partial \Omega^{e}} \stackrel{\leftrightarrow}{\mathbf{f}} \cdot \vec{n} \phi_{j} \mathrm{~d} S-\int_{\Omega^{e}} \stackrel{\leftrightarrow}{\mathbf{f}}(\mathbf{q}) \cdot \vec{\nabla} \phi_{j} \mathrm{~d} \Omega-\int_{\Omega^{e}} \mathbf{s} \phi_{j} \mathrm{~d} \Omega .
$$

For steady state, $\mathfrak{r}(\mathbf{q})=0$, the non-isolated truncation error becomes

$$
\tau^{N}=\mathfrak{r}^{N}\left(\mathbf{q}^{N}+\mathbf{I}^{N}\left(\boldsymbol{\epsilon}^{N}\right)\right)
$$

The discrete partial differential operator in discrete (sampled) form is derived from (2.13) as

$$
\mathfrak{R}^{N}\left(\mathbf{Q}^{N}\right)=\mathbf{H}\left(\mathbf{Q}^{N}\right)-\underline{\mathbf{M S}},
$$

where $\mathfrak{R}^{N}$ contains the sampled values of $\mathfrak{r}^{N}$ in all the nodes of the domain. The steady non-isolated truncation error is then simply

$$
\mathscr{T}^{N}=\mathfrak{R}^{N}\left(I^{N} \mathbf{q}\right)=\mathbf{H}\left(\boldsymbol{I}^{N} \mathbf{q}\right)-\underline{\mathbf{M S}} .
$$

The dependence of the non-isolated truncation error on the discretization error is obtained by using Definition 7.3 and expanding (7.26) as a Taylor series,

$$
\mathscr{T}^{N}=\left.\frac{\partial \mathfrak{R}^{N}}{\partial \mathbf{Q}^{N}}\right|_{\mathbf{Q}^{N}} \mathscr{E}^{N}+\mathscr{O}\left(\left(\epsilon^{N}\right)^{2}\right),
$$


where $\mathscr{E}^{N}$ is the generic vector containing the sampled values of $\boldsymbol{\epsilon}^{N}$.

Taking into account (7.27) and (7.20), and based on previous numerical results $[176,177]$, Kompenhans et al. [110] showed that the truncation error in an element is bounded by

$$
\left\|\left.\tau^{N}\right|_{\Omega^{e}}\right\|_{\infty} \leq \sum_{i=1}^{d} C_{i}^{e} e^{-N_{i}^{e} \eta_{i}^{e}}+\left\|\tau_{\partial \Omega^{e}}\right\|_{\infty} .
$$

This expression was validated experimentally in $[110,111]$. The first term in (7.28) is the bound on the locally-generated truncation error, whereas the second term is the bound on the externally-generated truncation error that enters through the Riemann solver and does not depend on the local polynomial orders. The second term is a consequence of the dependence of the discretization error on the solution in other elements.

Definition 7.5 (Isolated truncation error) The isolated truncation error is defined as [176]

$$
\hat{\tau}^{N}=\hat{\mathfrak{r}}^{N}\left(\mathbf{I}^{N}(\mathbf{q})\right)-\mathfrak{r}(\mathbf{q}),
$$

where $\hat{\mathbf{r}}^{N}(\cdot)$ is the isolated discrete partial differential operator, which is derived in the same manner as $\mathfrak{r}^{N}(\cdot)$, but $\overleftrightarrow{\mathbf{f}}$ is not substituted by $\hat{\mathbf{f}}$ during the process (2.4).

Therefore, in steady-state, the sampled form of the discrete isolated partial differential operator is

$$
\hat{\mathscr{T}}^{N}=\hat{\mathfrak{R}}^{N}\left(I^{N} \mathbf{q}\right)=\hat{\mathbf{H}}\left(I^{N} \mathbf{q}\right)-\underline{\mathbf{M S}},
$$

where the elemental contribution to the discrete isolated operator is

$$
\hat{\mathbf{h}}^{e}\left(\mathbf{Q}^{e}\right)_{j}=-\int_{\Omega^{e}}^{N} \stackrel{\leftrightarrow}{\mathbf{f}} \cdot \vec{\nabla} \phi_{j}^{e} \mathrm{~d} \Omega^{e}+\int_{\partial \Omega^{e}}^{N} \overleftrightarrow{\mathbf{f}}^{N} \cdot \vec{n} \phi_{j}^{e} \mathrm{~d} S^{e} .
$$

Using $\overleftrightarrow{\mathbf{f}}$ instead of $\hat{\mathbf{f}}$ eliminates the influence of the neighboring elements and boundaries in the truncation error of each element. Therefore, there is no externallygenerated isolated truncation error.

The spectral convergence of the isolated truncation error is similar to the nonisolated truncation error $[176,177]$ and can be expressed as

$$
\left\|\left.\hat{\tau}^{N}\right|_{\Omega^{e}}\right\|_{\infty} \leq \sum_{i=1}^{d} C_{i}^{e} e^{-N_{i}^{e} \eta_{i}^{e}}
$$

To simplify notation, from now on the expressions that do not use the hat notation are valid for both the non-isolated and the isolated truncation errors, unless explicitly stated. 


\subsubsection{Isolated Truncation Error Dependence on the Interpolation Error}

According to Definition 7.5 and (7.31), the steady isolated truncation error in the DGSEM can be expressed for any basis function $\phi_{j}$ in an element $e$ as

$$
\left.\hat{\tau}_{j}^{N}\right|_{\Omega^{e}}=\hat{\mathfrak{r}}\left(\mathbf{I}^{N} \mathbf{q}\right)=\int_{\partial \Omega}^{N} \overleftrightarrow{\mathbf{f}}^{N}(\mathbf{q}) \cdot \vec{n} \phi_{j} \mathrm{~d} S-\int_{\Omega}^{N} \overleftrightarrow{\mathbf{f}}^{N}(\mathbf{q}) \cdot \vec{\nabla} \phi_{j} \mathrm{~d} \Omega-\int_{\Omega}^{N} \mathbf{s}^{N} \phi_{j} \mathrm{~d} \Omega,
$$

where the superindex $N$ on the integrals indicates that they are approximated with a Gaussian quadrature of order $N$ and the superindex $e$ has been dropped for readability. Since the DGSEM is a collocation method, the value computed with (7.33) corresponds to the isolated truncation error on the node of the basis function $\phi_{j}$. The terms $\mathbf{s}^{N}$ and $\overleftrightarrow{\mathbf{f}}^{N}$ can be expressed in terms of the interpolation error as

$$
\overleftrightarrow{\mathbf{f}}^{N}=\mathbf{I}^{N}(\stackrel{\leftrightarrow}{\mathbf{f}}(\mathbf{q}))=\overleftrightarrow{\mathbf{f}}(\mathbf{q})-\underset{\mathbf{\varepsilon}}{\stackrel{\mathrm{f}}{N}}, \quad \mathbf{s}^{N}=\mathbf{I}^{N}(\mathbf{s})=\mathbf{s}-\varepsilon_{\mathbf{s}}^{N}
$$

Inserting (7.34) into (7.33), summing by parts, and expressing everything with discrete $L_{2}(\Omega)$ inner product notation we obtain,

$$
\left.\left.\hat{\tau}^{N}\right|_{\Omega^{e}}=\left\langle\boldsymbol{\varepsilon}_{\mathrm{s}}^{N}, \phi\right\rangle_{\Omega^{e}}^{N}-\langle\vec{\nabla} \cdot \underset{\mathrm{\varepsilon}}{\stackrel{\leftrightarrow}{\mathrm{f}}}, \phi\rangle\right\rangle_{\Omega^{e}}^{N}+\mathscr{O}\left(e_{\Omega^{e}}^{N}\right),
$$

where $\langle\cdot, \cdot\rangle_{\Omega^{e}}^{N}$ stands for the $L_{2}$ inner product operator evaluated with a quadrature of order $N$ in the domain $\Omega^{e}$. The first term on the right-hand side of (7.35) vanishes since the value of $\varepsilon_{\mathrm{s}}^{N}$ is zero on the quadrature nodes (the DGSEM is a collocation method). Furthermore, it is reasonable to neglect the quadrature error since it is of a lower magnitude than the value of the integral. Therefore, we obtain

$$
\left.\hat{\tau}^{N}\right|_{\Omega^{e}} \approx-\langle\vec{\nabla} \cdot \underset{\mathrm{f}}{\stackrel{\leftrightarrow}{\leftrightarrow}}, \phi\rangle_{\Omega^{e}}^{N}
$$

The dependence of the isolated truncation error on the interpolation error of the fluxes inside an element $\left(\stackrel{\leftrightarrow}{\boldsymbol{\varepsilon}} \begin{array}{c}N \\ \mathbf{f}, e\end{array}\right)$, originally obtained by Rubio et al. [176], shows that $\hat{\tau}$ indeed depends only on the discrete representation of the numerical solution in the element $e$.

Rubio et al. [176] pointed out that, since the isolated truncation error is not affected by neighbors' errors, it might be a better sensor for adaptation algorithms for hyperbolic PDEs than its non-isolated counterpart or the discretization error. Equation (7.36) shows that the isolated truncation error acts as a source term for the interpolation error. In consequence, decreasing the isolated truncation error reduces the interpolation error.

\section{$7.4 \tau$-Estimation Method}

Since the exact solution to the problem is generally not available, we use an estimate for (7.26) and (7.30). The $\tau$-estimation method makes use of an approximate 
solution on a reference mesh of order $P>N$ instead of the exact one. The most straightforward approach is to converge this high-order solution, $\mathbf{Q}^{P}$, to a low residual near machine round-off. This is known as the a posteriori approach and can be expressed in discrete form for steady state as

$$
\mathscr{T}^{N} \approx \mathscr{T}_{P}^{N}=\mathfrak{R}^{N}\left(\underline{I}_{P}^{N} \mathbf{Q}^{P}\right)=\mathbf{H}^{N}\left(\mathbf{Q}^{P}\right)-\underline{\mathbf{M}}^{N} \mathbf{S}^{N},
$$

where $\underline{I}_{P}^{N}$ is an interpolation operator from order $P$ to order $N$.

In practice, it can be very expensive to converge the solution on a high-order mesh of order $P$ down to machine round-off. Therefore, a non-converged solution, $\tilde{\mathbf{Q}}^{P}$ is usually used. This is known as the quasi-a priori approach and can be formulated in two ways:

- The quasi a-priori approach without correction simply replaces $\mathbf{Q}^{P}$ by $\tilde{\mathbf{Q}}^{P}$ to get, for steady state,

$$
\left.\mathscr{T}_{P}^{N}\right|_{\text {quasi }}=\mathfrak{R}^{N}\left(\underline{I}_{P}^{N} \tilde{\mathbf{Q}}^{P}\right)=\mathbf{H}^{N}\left(\tilde{\mathbf{Q}}^{P}\right)-\underline{\mathbf{M}}^{N} \mathbf{S}^{N} .
$$

Note that (7.38) is valid for both the isolated (inserting the hats) and the non-isolated truncation error.

It is important to bear in mind that an additional error is being carried into the estimate. Namely, the non-converged solution has an associated iteration error,

$$
\mathbf{Q}^{P}=\tilde{\mathbf{Q}}^{P}+\mathscr{E}_{\mathrm{it}}^{P}
$$

Therefore, (7.38) is actually

$$
\begin{aligned}
\left.\mathscr{T}_{P}^{N}\right|_{\text {quasi }} & =\mathfrak{R}_{P}^{N}\left(\underline{\mathbf{I}}_{P}^{N} \mathbf{Q}^{P}\right)-\left.\frac{\partial \mathfrak{R}^{N}}{\partial \mathbf{Q}^{N}}\right|_{\tilde{\mathbf{Q}}^{N}} \underline{\mathbf{I}}_{P}^{N} \mathscr{E}_{\mathrm{it}}^{P}+\mathscr{O}\left(\left\|\mathscr{E}_{\mathrm{it}}^{P}\right\|^{2}\right) \\
& =\mathscr{T}_{P}^{N}-\left.\frac{\partial \boldsymbol{R}^{N}}{\partial \mathbf{Q}^{N}}\right|_{\underline{I}_{P}^{N} \tilde{\mathbf{Q}}^{p}} \underline{\mathbf{I}}_{P}^{N} \mathscr{E}_{\mathrm{it}}^{P}+\mathscr{O}\left(\left\|\mathscr{E}_{\mathrm{it}}^{P}\right\|^{2}\right),
\end{aligned}
$$

where the additional terms on the right-hand-side correspond to the errors of the quasi a-priori approach. To get an approximation of the truncation error down to a value of $\tau_{\max }$, the high-order solution must be converged down to a residual $\tilde{\mathfrak{R}}^{P}\left(\tilde{\mathbf{Q}}^{P}\right) \leq \tau_{\max } / F$, so that the iteration error is not so high as to greatly contaminate the estimation. A typical value of $\mathrm{F}$ is 10 [110].

- The quasi a-priori approach with correction approximates the second term of the Taylor expansion in (7.40) and uses it to correct the quasi a-priori estimate. The iteration error can be easily estimated by doing a Taylor expansion on the high-order residual equation,

$$
\mathfrak{R}^{P}\left(\mathbf{Q}^{P}\right)=\mathfrak{R}^{P}\left(\tilde{\mathbf{Q}}^{P}\right)+\left.\frac{\partial \mathfrak{R}}{\partial \mathbf{Q}^{P}}\right|_{\tilde{\mathbf{Q}}^{P}} \mathscr{E}_{i t}^{P}+\mathscr{O}\left(\left\|\mathscr{E}_{\mathrm{it}}^{P}\right\|^{2}\right)
$$


Therefore, the quasi a-priori approach with correction becomes

$$
\left.\mathscr{T}_{P}^{N}\right|_{\text {quasi corr }}=\mathfrak{R}_{P}^{N}\left(\underline{\mathbf{I}}_{P}^{N} \tilde{\mathbf{Q}}^{P}\right)+\left.\left.\frac{\partial \mathfrak{R}^{N}}{\partial \mathbf{Q}^{N}}\right|_{\underline{I}_{P}^{N} \tilde{\mathbf{Q}}^{P}} \underline{\mathbf{I}}_{P}^{N} \frac{\partial \mathfrak{R}^{P}}{\partial \mathbf{Q}^{P}}\right|_{\tilde{\mathbf{Q}}^{N}} ^{-1} \mathfrak{R}^{P}\left(\tilde{\mathbf{Q}}^{P}\right) .
$$

Again, since the converged solution of order $P$ is not used, a good approximation of the truncation error down to a value of $\tau_{\max }$ is achieved by converging Q̃ to a specified residual. According to Kompenhans et al. [110], in the noncorrected quasi a-priori approach that residual is $\tilde{\mathfrak{R}}^{P}\left(\tilde{\mathbf{Q}}^{P}\right) \leq\left(\tau_{\max } / F\right)^{1 / 2}$.

For compactness, the notation of the rest of this work omits the interpolation matrix so that $\mathfrak{R}^{N}\left(\underline{\mathbf{I}}_{P}^{N} \mathbf{Q}^{P}\right) \leftarrow \mathfrak{R}^{N}\left(\mathbf{Q}^{P}\right)$.

In what remains of this thesis, the quasi a-priori approach without correction is used. Namely, the computational cost of computing the Jacobian matrices for the reference mesh $(P)$ and the sub-meshes $(N)$, and inverting the former, is generally much higher than just doing the non-corrected approach $[110,111]$.

Finally, note that the truncation error estimate can be easily performed for $p$ anisotropic discretizations of $d$ dimensions. For instance, in a 2D anisotropic case, (7.38) can be rewritten as

$$
\mathscr{T}_{P_{1} P_{2}}^{N_{1} N_{2}}=\mathbf{H}^{N_{1} N_{2}}\left(\tilde{\mathbf{Q}}^{P_{1} P_{2}}\right)-\underline{\mathbf{M}}^{N_{1} N_{2}} \mathbf{S}^{N_{1} N_{2}} .
$$

This idea is further developed in Chapter 8.

\subsection{Nonconforming Discretizations and Mortar Spaces}

For a $p$-nonconforming DGSEM discretization to be consistent, the numerical fluxes are evaluated on the nodes of a mortar space $\Xi$ that connects the faces of two elements [116], a similar approach as the one proposed by Mavriplis [141] for spectral elements. Figure 7.1 illustrates the problem for two quadrilateral elements that are connected through their faces $L$ and $R$. The solution on faces $L$ and $R$ are, respectively,

$$
\mathbf{Q}^{L} \in \mathscr{P}^{N_{2}^{L}}, \quad \mathbf{Q}^{R} \in \mathscr{P}^{N_{1}^{R}} .
$$

The mortar space stores the solution on both sides, $\Psi^{L}$ and $\Psi^{R}$, so that the numerical flux can be evaluated in each node of the mortar space, $\hat{\mathbf{f}}\left(\boldsymbol{\psi}_{i}^{L}, \boldsymbol{\psi}_{i}^{R}, \vec{n}\right)$. Therefore, it must contain the left and right polynomial spaces, $\mathscr{P}^{N_{2}^{L}}, \mathscr{P}_{1}^{N_{1}^{R}} \subseteq \mathscr{P} \Xi$. The usual choice for the mortar polynomial order is simply the maximum between the left and right spaces, $N^{\Xi}=\max \left(N_{2}^{L}, N_{1}^{R}\right)$. In this case, the projection from the left element to the mortar is the identity matrix,

$$
L^{\Xi}=\underline{\mathbf{I}}_{N^{\Xi}} .
$$




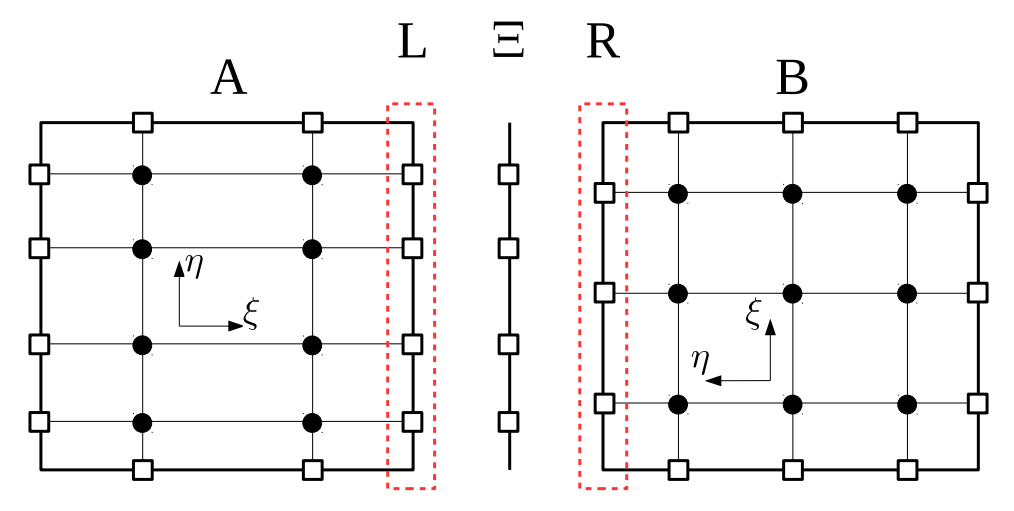

Figure 7.1: Representation of the mortar space $\Xi$ that connects the faces $L$ and R.

For the right element, an $L^{2}$ projection is used,

$$
\int_{\Xi}\left(\psi^{R}-\mathbf{q}^{R}\right) \ell_{i}^{\Xi} \mathrm{d} S=0, \quad \text { with } i=0, \ldots, N^{\Xi},
$$

which ensures conservation of the quantity q. The right projection matrix is

$$
\underline{\mathbf{I}}_{R}^{\Xi}=\underline{\mathbf{M}}^{-1} \underline{\mathbf{S}}^{R}
$$

where

$$
S_{i, j}^{R}=\int_{\Xi} \ell_{j}^{R} \ell_{i}^{\Xi} \mathrm{d} S
$$

and

$$
M_{i, j}=\int_{\Xi} \ell_{j}^{\Xi} \ell_{i}^{\Xi} \mathrm{d} S .
$$

The projection operators from the mortar space to the elements are computed in the same way. These ideas can be easily extended to 3D with some additional considerations: the polynomial space must contain the space of tensor-product basis functions on the faces and additional rotations must be taken into account.

This method has been extended to connect $h$-nonconforming DG elements with hanging nodes [116], but this is not explained in this thesis since we focus on $p$ adaptation strategies. 



$\Gamma_{\text {Chapter }} \mathbf{O}$

\section{Truncation Error Estimation in the p-Anisotropic DGSEM}

\section{Contents}

8.1 Introduction . . . . . . . . . . . . . . . . . . 114

8.2 Low-Order Extrapolation . . . . . . . . . . . . . 115

8.2.1 Analysis of the Low-Order Extrapolation . . . . . . . . . . 117

8.3 A New Anisotropic Truncation Error Estimator . . . . . . . . 118

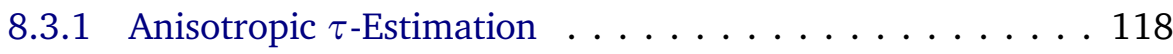

8.3.2 Convergence Behavior of the Anisotropic Truncation Error 124

8.3.3 High-Order Extrapolation of the Truncation Error Estima-

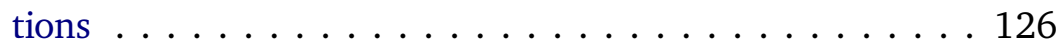

8.3.4 Theoretical Comparison of the New Anisotropic $\tau$-Esti- mation with Previous Approaches . . . . . . . . . . . 126

8.3.5 Additional Considerations . . . . . . . . . . . . . . 128

8.4 Validation of the Anisotropic $\tau$-Estimation Method . . . . . 129

8.4.1 Truncation Error Maps and Number of Degrees of Freedom 130

8.4.2 Comparison with Previous Methods . . . . . . . . . . 132

8.4.3 p-Anisotropic Adaptation using the New Non-Isolated and Isolated Truncation Error Estimators . . . . . . . . . . 135

8.4.4 Anisotropic Error Estimation under High-Order Mappings 137

8.5 Final Remarks $\ldots \ldots \ldots \ldots \ldots \ldots \ldots \ldots \ldots$ 


\subsection{Introduction}

One of the most important advantages of the truncation error is that, due to its spectral convergence behavior, it is possible to extrapolate the estimated values, $\tau^{N}$ (where $N<P$ ), to predict the truncation error of approximate solutions with a higher order than the reference mesh, $N \geq P$. This allows reference solutions that are not too expensive to compute. The extrapolation process is straightforward for $p$-isotropic discretizations. However, for $p$-anisotropic discretizations, the extrapolation is not trivial.

As was shown in Section 7.4, the $\tau$-estimation method can be directly used to estimate the truncation error of anisotropic discretizations. Kompenhans et al. [110] proposed an approach that was shown to be useful for anisotropic $p$-adaptation in 2D approximations $[110,111]$. In light of the analysis presented in this chapter, it can be concluded that the method of Kompenhans et al. [110] can be considered as a low-order extrapolation of the truncation error (see Section 8.2), and that, although it tends to under-predict the truncation error for high orders, it introduces a "secure" way to select polynomial orders in some extrapolated regions, in the sense that it over-predicts the polynomial order needed.

In this chapter, we present a novel anisotropic truncation error estimator derived from the $\tau$-estimation procedure for the DGSEM, a work that has been reported by the author in [180]. The work on high-order $\tau$-estimators by Rubio et al. [175, 176, 177] and Kompenhans et al. [110, 111] is extended to formulate a new anisotropic truncation error estimator that exploits the tensor product basis expansion of the DGSEM so that the total truncation error is calculated as a sum of its directional components. The new error estimator is shown to be suitable to perform anisotropic $p$-adaptation, and to have two main advantages over existing truncation error estimators: first, that it requires fewer operations to estimate the truncation error of all possible combinations of polynomial orders; and second, that it yields more accurate (high-order) extrapolations of the truncation error for discretizations of a higher order than the reference mesh. These features allow the use of reference meshes of a lower polynomial order, hence reducing the computational cost. Although the present work is for a nodal (collocation) DG scheme, the proposed adaptation strategy is also suited for modal (hierarchical bases) DG approaches.

The chapter is organized as follows. In Section 8.2, the state-of-the-art of techniques for extrapolating the truncation error of anisotropic discretizations using the $\tau$-estimation method is reviewed. In Section 8.3, the new anisotropic $\tau$-estimator is formulated and analyzed. Finally, in Section 8.4, the new estimation method is validated and compared with previous strategies by means of a manufactured solution test case for the compressible Navier-Stokes equations on straight and curved meshes. 


\subsection{Low-Order Extrapolation of the Truncation Error Estimations}

In this section, we review the method proposed by Kompenhans et al. [110] to extrapolate the truncation error estimates of $p$-anisotropic discretizations. This method was successfully used to perform a $p$-adaptation strategy $[110,111]$. We show that their strategy can be classified as a low-order extrapolation. To do so, let us first introduce the concept of a truncation error map.

Definition 8.1 (Truncation error map) A truncation error map is the (graphical) representation of the truncation error behavior inside an element with respect to the polynomial order, i.e. a $(d+1)$-dimensional plot of $\log \left\|\tau^{N}\right\|_{\infty}$ as a function of the polynomial order in every direction of the reference element $N=\left(N_{1}, \cdots, N_{d}\right)$, where $d$ is the number of dimensions.

As we want to generate an approximation to the truncation error map with the $\tau$-estimation method (Section 7.4) and extrapolate it to higher polynomial orders, two regions of the estimated map can be distinguished:

Definition 8.2 (Inner truncation error map) The inner truncation error map is the portion of the truncation error map that is generated by the $\tau$-estimation method. If a reference mesh of order $P$ is used for the estimate, the inner truncation error map is the region estimated for $N_{i}<P_{i}$.

Definition 8.3 (Outer truncation error map) The outer truncation error map is the portion of the truncation error map that is extrapolated from the inner truncation error map. If a reference mesh of order $P$ is used for the estimation, the outer truncation error map is the region estimated for $N_{i} \geq P_{i}$.

Since the numerical solution on a reference mesh of order $P$ requires significant computational resources, it is of interest to have an accurate and efficient way to generate the outer truncation error map.

Moreover, because of the spectral convergence of the truncation error in the asymptotic range, the one-dimensional (or isotropic $d$-dimensional) map turns out to be a discrete scatter plot of points that describe a linear function with a negative slope (the convergence rate $\eta$ ), as shown in Figure 8.1(a).

Kompenhans et al. [110] developed a strategy to extrapolate the inner truncation error map in 2D approximations, and used the truncation error map to adapt the polynomial orders of a given mesh using a specified maximum permitted error threshold, $\tau_{\max }$. The method to estimate the map consists of four steps:

1. Generate an inner map for $N_{i}<P_{i}$ using (7.43). This requires $n_{\text {eval }}$ evaluations of the discrete partial differential operator $\mathfrak{R}^{N}$, where

$$
n_{\text {eval }}=\prod_{i=1}^{d}\left(P_{i}-1\right) \text {. }
$$




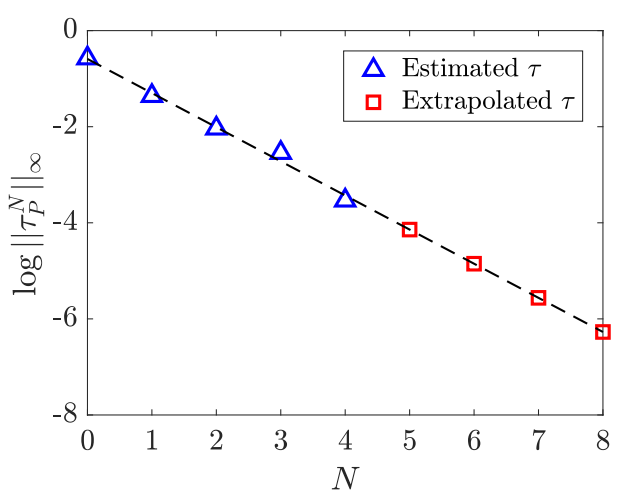

(a) One-dimensional map

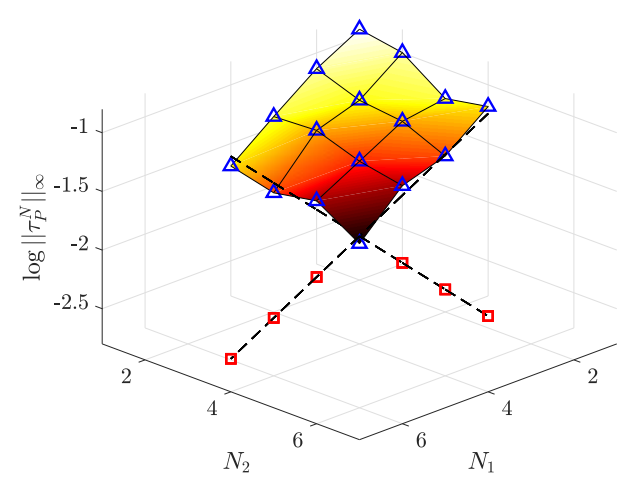

(b) Two-dimensional map used by Kompenhans et al. [110]

Figure 8.1: One- and two-dimensional truncation error maps constructed with $P=$ 5 showing estimated and extrapolated values for a toy problem.

The estimated points of the inner map are marked as blue triangles in Figure 8.1 .

2. Use the inner map to look for a combination of polynomial orders that fulfills the specified error threshold. If a combination fulfills $\tau_{\max }$, adapt the polynomial order and exit the estimation/adaptation process. Otherwise, additional considerations are required (Steps 3 and 4).

3. Compute $\log \left\|\tau^{N_{i} N_{j}}\right\|_{\infty}$ and perform a linear regression analysis on it in the direction $i$ to describe the behavior of $\log \|\tau\|_{\infty}$ as a function of $N_{i}$, with $N_{j}=$ $P_{j}-1$. The result of the linear regression is marked with a dashed line in Figure 8.1.

4. Use the linear regression to generate part of the outer truncation error map for $N_{i} \geq P_{i}$, and select the value of $N_{1}$ and $N_{2}$ independently from these extrapolations. The extrapolated values of the truncation error are marked with red squares in Figure 8.1. In other words, $N_{1}$ and $N_{2}$ are selected so that the values of both red squares fall below the specified threshold, $\tau_{\max }$.

This procedure is performed for every element of the mesh in each of the reference space directions. For further details, refer to the original paper by Kompenhans et al. [109, 110] and to our example in Section 8.4.2.

Hereinafter, the method by Kompenhans et al. shall be referred to as the loworder extrapolation method, since it supposes that the truncation error map, $\log \|\tau\|_{\infty}$, has a linear behavior. In light of the analysis in Section 8.3, we will be able to formulate a high order extrapolation method that provides extrapolated estimations with increased accuracy. 


\subsubsection{Analysis of the Low-Order Extrapolation}

Two remarks can be made about the four-step procedure of Kompenhans et al. [110]:

Remark 1 Steps 3 and 4 assume that the spectral convergence observed in 1D extends to higher dimensions along iso- $N_{i}$ lines of the truncation error map.

Remark 2 For the non-isolated truncation error, the spectral convergence behavior shown in Figure 8.1 can only be expected for the locally-generated component, as can be interpreted from (7.28). This means that the linear regression (Step 3) may not be an accurate method to extrapolate the error if the truncation error in neighboring elements is high, i.e., if the $\tau$-estimation procedure is not performed element-wise while keeping the polynomial order in other elements sufficiently ${ }^{1}$ high.

As stated in Remark 1, the extrapolation procedure assumes that the truncation error decreases exponentially along iso- $N_{i}$ lines of the truncation error map. That is the same as saying that the truncation error map is a plane for $d=2$, and in general that it is a hyperplane of dimension $d+1$. In Figure 8.2 we present an illustration that resembles the hyperplane behavior in two dimensions for perfect spectral convergence. The method constructs $d$ iso- $N_{i}$ lines on the hyperplane, which should contain the values of the truncation error for $N_{j}=P_{j}-1 \forall N_{i}$ (red line with triangular markers and black line with circular markers in Figure 8.2). In that scenario, selecting $N_{i}$ independently can be regarded as a secure criterion, since in the hyperplane we have:

- In 2D:

$$
\begin{aligned}
& \left\|\tau^{N_{1} N_{2}}\right\|_{\infty} \leq\left\|\tau^{P_{1} N_{2}}\right\|_{\infty}, \\
& \left\|\tau^{N_{1} N_{2}}\right\|_{\infty} \leq\left\|\tau^{N_{1} P_{2}}\right\|_{\infty} .
\end{aligned}
$$

- In 3D:

$$
\begin{aligned}
& \left\|\tau^{N_{1} N_{2} N_{3}}\right\|_{\infty} \leq\left\|\tau^{P_{1} N_{2} N_{3}}\right\|_{\infty}, \\
& \left\|\tau^{N_{1} N_{2} N_{3}}\right\|_{\infty} \leq\left\|\tau^{N_{1} P_{2} N_{3}}\right\|_{\infty}, \\
& \left\|\tau^{N_{1} N_{2} N_{3}}\right\|_{\infty} \leq\left\|\tau^{N_{1} N_{2} P_{3}}\right\|_{\infty} .
\end{aligned}
$$

for $N_{i} \geq P_{i}$ (see Figure 8.2).

\footnotetext{
${ }^{1}$ Sufficiently high does not necessarily mean that the polynomial order of the other elements must be kept in $P$, but that it must be high enough so that the externally-generated contributions to the truncation error are less than the internally-generated ones.
} 


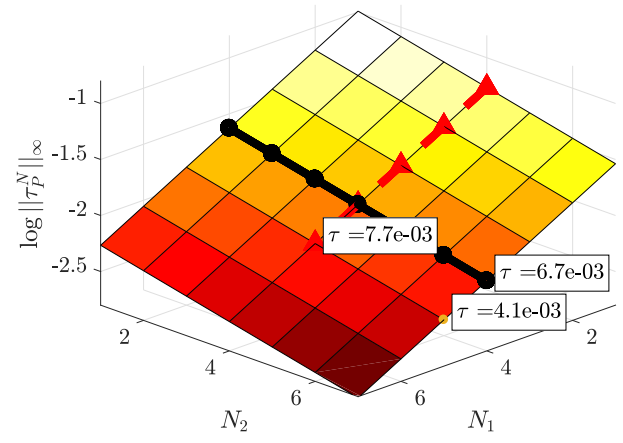

Figure 8.2: Hyperplane behavior of the truncation error of a toy problem.

\subsection{A New Anisotropic Truncation Error Estimator}

In this section, we present a new anisotropic truncation error estimator, discuss some of its properties and compare it with the truncation error estimators that have been used in the literature to perform anisotropic $p$-adaptation. The formulation of this new estimator uses a mathematical proof found in Section 8.3.1. In Section 8.3.2, we analyze the convergence behavior of the anisotropic estimator. In Section 8.3.3, we describe how the new estimator can be used to extrapolate the truncation error, and prove that it is superior to existing $\tau$-estimators at obtaining these extrapolations.

\subsubsection{Anisotropic $\tau$-Estimation}

The anisotropic $\tau$-estimator is a generalization of the $\tau$-estimation method reviewed in Section 7.4. For the sake of readability and without loss of generality, all the mathematical statements in this section are for 2D formulations, where $N=\left(N_{\xi}, N_{\eta}\right)=\left(N_{1}, N_{2}\right)$ are the polynomial orders in the two directions of the reference element. However, all the statements and proofs can be directly generalized to $d$ dimensions.

\section{Assumptions}

Following assumptions are a consequence of the tensor product basis functions of the DGSEM and hold for sufficiently smooth solutions in the asymptotic range. The assumptions are:

(a) The truncation error has an anisotropic behavior and, therefore, can be decoupled in its directional components:

$$
\tau^{N_{1} N_{2}} \approx \tau_{1}^{N_{1} N_{2}}+\tau_{2}^{N_{1} N_{2}}
$$


Here, it is important to note that $\tau_{i}$ is the projection of the global truncation error, $\tau$, into the local direction of the reference space, $i$.

From now on, unless explicitly stated, a numeric subscript on the truncation error refers to directional components of the error, e.g. $\tau_{1}^{N}, \tau_{2}^{N}, \tau_{3}^{N}$.

(b) The locally-generated truncation error in each direction depends only on the polynomial order in that direction:

$$
\tau_{\Omega, i}^{N_{1} N_{2}} \approx \tau_{\Omega, i}^{N_{1} N_{2}}\left(N_{i}\right)
$$

where the first subscript specifies that we are considering the locally-generated error, and the second subscript is the directional component.

Assumptions (a) and (b) were numerically validated by Rubio et al. [176, 177] based on previous results by Georgoulis [83]. Furthermore, assumption (b) fits the anisotropic spectral convergence behavior of the truncation error (equations (7.28) and (7.32)).

Proposition 1 For sufficiently smooth solutions, the truncation error of a DGSEM discretization of order $\left(N_{1}, N_{2}\right)$ can be approximated from a semi-converged solution of order $\left(P_{1}, P_{2}\right)$, where $P_{i}>N_{i}$, as the sum of the directional $\tau$-estimations obtained by coarsening in the different reference coordinate directions:

$$
\tau^{N_{1} N_{2}} \approx \tau_{P_{1} P_{2}}^{N_{1} P_{2}}+\tau_{P_{1} P_{2}}^{P_{1} N_{2}}
$$

Proof. 1 (a) This proof is specific for the isolated truncation error. We refer to Proof 1(b) for the demonstration that proposition 1 also holds for the non-isolated truncation error under additional assumptions.

Let us note that assumptions (a) and (b) are consistent with the dependence of the isolated truncation error on the interpolation error (7.36) and the anisotropic behavior of the latter (7.6).

We start by obtaining the analytical expression for the isolated $\tau$-estimation. To that end, we use the same procedure as in Section 7.3.5.1. The estimate of the isolated truncation error in the DGSEM can be expressed for any basis function $\phi$ in an element e as

$$
\left.\hat{\tau}_{P}^{N}\right|_{\Omega^{e}}=\hat{\mathfrak{r}}\left(\mathbf{I}^{N}\left(\mathbf{q}^{P}\right)\right)=\int_{\partial \Omega^{e}}^{N} \overleftrightarrow{\mathbf{f}}^{N}\left(\mathbf{q}^{P}\right) \cdot \vec{n} \phi \mathrm{d} S-\int_{\Omega^{e}}^{N} \overleftrightarrow{\mathbf{f}}^{N}\left(\mathbf{q}^{P}\right) \cdot \vec{\nabla} \phi \mathrm{d} \Omega-\int_{\Omega^{e}}^{N} \mathbf{s}^{N} \phi d \Omega
$$

Instead of the exact solution to the problem, $\mathbf{q}$, we use a solution on a higher order mesh, $\mathbf{q}^{P}$. Furthermore, note that the subindex $j$ was dropped for readability. However, bear in mind that (8.5) is the isolated truncation error for the degree of freedom $j$ that corresponds to the basis function $\phi_{j} \leftarrow \phi$. 
Using the definition of interpolation error (7.3) and discretization error (7.9), the flux is

$$
\overleftrightarrow{\mathbf{f}}^{N}=\mathbf{I}^{N}\left(\overleftrightarrow{\mathbf{f}}\left(\mathbf{q}^{P}\right)\right)=\overleftrightarrow{\mathbf{f}}\left(\mathbf{q}^{P}\right)-\underset{\mathbf{\varepsilon}}{\stackrel{\leftrightarrow}{N}}=\overleftrightarrow{\mathbf{f}}(\mathbf{q})-\left.\frac{\partial \mathbf{f}}{\partial \mathbf{q}}\right|_{\mathbf{q}} \boldsymbol{\epsilon}^{P}-\underset{\mathbf{\varepsilon}}{\stackrel{\leftrightarrow}{N}}+\mathscr{O}\left(\left(\boldsymbol{\epsilon}^{P}\right)^{2}\right)
$$

and the source term is

$$
\mathbf{s}^{N}=\mathbf{I}^{N}(\mathbf{s})=\mathbf{s}-\boldsymbol{\varepsilon}_{\mathbf{s}}^{N} .
$$

Inserting equations (8.6) and (8.7) into (8.5), and summing by parts again, we obtain

$$
\left.\hat{\tau}_{P}^{N}\right|_{\Omega^{e}}=-\langle\vec{\nabla} \cdot \underset{\mathbf{\varepsilon}}{\stackrel{\leftrightarrow}{\mathbf{f}}}, \phi\rangle_{\Omega^{e}}^{N}-\left\langle\left.\vec{\nabla} \cdot \frac{\partial \overleftrightarrow{\mathbf{f}}}{\partial \mathbf{q}}\right|_{\mathbf{q}} \boldsymbol{\epsilon}^{P}, \phi\right\rangle_{\Omega^{e}}^{N}+\mathscr{O}\left(\left(\boldsymbol{\epsilon}^{P}\right)^{2}\right)+\mathscr{O}\left(e_{\Omega^{e}}^{N}\right) .
$$

Let us note that although the isolated truncation error of an element does not depend on external sources, its approximation by $\tau$-estimation is affected by the discretization error of the reference mesh, $\epsilon^{P}$. This translates into a slight influence of external (upwind) errors transmitted through the Riemann solver. In the twodimensional case and coarsening in only one direction (here the direction (1)), (8.8) becomes

$$
\left.\hat{\tau}_{P_{1} P_{2}}^{N_{1} P_{2}}\right|_{\Omega^{e}}=-\left\langle\vec{\nabla} \cdot \underset{\mathbf{\varepsilon}}{\stackrel{\leftrightarrow}{N_{1} P_{2}}}, \phi\right\rangle_{\Omega^{e}}^{N_{1} P_{2}}-\left\langle\left.\vec{\nabla} \cdot \frac{\partial \overleftrightarrow{\mathbf{f}}}{\partial \mathbf{q}}\right|_{\mathbf{q}} \boldsymbol{\epsilon}^{P_{1} P_{2}}, \phi\right\rangle_{\Omega^{e}}^{N_{1} P_{2}}+\mathscr{O}\left(\left(\boldsymbol{\epsilon}^{P_{1} P_{2}}\right)^{2}\right)+\mathscr{O}\left(e_{\Omega^{e}}^{N_{1} P_{2}}\right) .
$$

We now rewrite (8.9), decoupling the interpolation error in directional components and taking into account that $\varepsilon_{2}^{N_{1} P_{2}}=\varepsilon_{2}^{P_{1} P_{2}}$ (7.5),

$$
\begin{aligned}
& \hat{\tau}_{P_{1} P_{2}}^{N_{1} P_{2}}=-\left\langle\vec{\nabla} \cdot \underset{\mathbf{\varepsilon}, 1}{\stackrel{\leftrightarrow}{\leftrightarrow} N_{1} P_{2}}, \phi\right\rangle_{\Omega^{e}}^{N_{1} P_{2}}-\left\langle\vec{\nabla} \cdot \underset{\mathbf{\varepsilon}, \stackrel{\leftrightarrow}{f}, 2_{1}^{P_{1} P_{2}}}{,}, \phi\right\rangle_{\Omega^{e}}^{N_{1} P_{2}} \\
& -\left\langle\left.\vec{\nabla} \cdot \frac{\partial \overleftrightarrow{\mathbf{f}}}{\partial \mathbf{q}}\right|_{\mathbf{q}} \boldsymbol{\epsilon}^{P_{1} P_{2}}, \phi\right\rangle_{\Omega^{e}}^{N_{1} P_{2}}+\mathscr{O}\left(\left(\boldsymbol{\epsilon}^{P_{1} P_{2}}\right)^{2}\right)+\mathscr{O}\left(e_{\Omega^{e}}^{N_{1} P_{2}}\right) .
\end{aligned}
$$

Note that all terms on the right-hand side of (8.10), except for the first one and the quadrature error, are of the order of errors on the higher-order mesh. If the solution is smooth, the discretization and interpolation errors on the high order mesh of order $P$ are smaller than the errors on the current mesh $N$, and their gradients are bounded. Therefore, and taking into account that we are coarsening in direction (1), for sufficiently smooth solutions we can expect the first term on the right-hand side to be the leading term. Simplifying, the directional $\hat{\tau}$-estimation provides

$$
\hat{\tau}_{P_{1} P_{2}}^{N_{1} P_{2}} \approx-\left\langle\vec{\nabla} \cdot \underset{\mathbf{\varepsilon}}{\stackrel{\leftrightarrow}{\leftrightarrow}, N_{1} P_{2}}, \phi\right\rangle_{\Omega^{e}}^{N_{1} P_{2}}
$$


On the other hand, inserting (7.5) into (7.36), the isolated truncation error of a discretization of order $N=\left(N_{1}, N_{2}\right)$ becomes

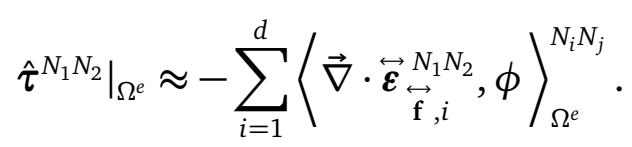

Notice, again, that the directional components of the interpolation error depend only on the polynomial order in the corresponding direction (7.5). Therefore, neglecting additional quadrature errors, we recover (8.4) for the isolated truncation error by combining equations (8.11) and (8.12),

$$
\hat{\tau}^{N_{1} N_{2}} \approx \hat{\tau}_{P_{1} P_{2}}^{N_{1} P_{2}}+\hat{\tau}_{P_{1} P_{2}}^{P_{1} N_{2}}
$$

We can conclude that when the $\tau$-estimation method is performed coarsening only in the direction $i$, the result is an approximation to the $i$-directional component of the truncation error, $\tau_{i}$. We can also arrive at this conclusion intuitively if we realize that $\mathbf{Q}^{P_{1} P_{2}}$ cannot be better than $\mathbf{Q}^{N_{1} P_{2}}$ at describing the solution in the direction (2).

Proposition 1 can be directly generalized to three dimensions to obtain

$$
\begin{aligned}
& \tau^{N_{1} N_{2} N_{3}} \approx \tau_{1}^{N_{1} N_{2} N_{3}}+\tau_{2}^{N_{1} N_{2} N_{3}}+\tau_{3}^{N_{1} N_{2} N_{3}} \\
& \tau^{N_{1} N_{2} N_{3}} \approx \tau_{P_{1} P_{2} P_{3}}^{N_{1} P_{2} P_{3}}+\tau_{P_{1} P_{2} P_{3}}^{P_{1} N_{2} P_{3}}+\tau_{P_{1} P_{2} P_{3} P_{2} N_{3}}^{P_{1}} .
\end{aligned}
$$

Proof. 1 (b) This is the demonstration that the non-isolated truncation error can be estimated anisotropically using Proposition 1. To do so, we need some additional assumptions.

\section{Additional Assumptions}

As in Section 8.3.1, the following assumptions are a consequence of the tensor product basis functions of the DGSEM and hold for sufficiently smooth solutions in the asymptotic range:

(c) The discretization error has an anisotropic behavior and, therefore, can be decoupled in directional components. For 2D,

$$
\boldsymbol{\epsilon}^{N_{1} N_{2}}=\boldsymbol{\epsilon}_{1}^{N_{1} N_{2}}+\boldsymbol{\epsilon}_{2}^{N_{1} N_{2}}
$$

As in (a), $\boldsymbol{\epsilon}_{i}$ is the projection of the global discretization error, $\boldsymbol{\epsilon}$, into a local direction of the reference space, $i$.

From now on, unless explicitly stated, a numeric subscript on the discretization error refers to directional components of the error, e.g. $\epsilon_{1}^{N}, \epsilon_{2}^{N}, \epsilon_{3}^{N}$.

(d) The locally-generated discretization error in each direction depends only on the polynomial order in that direction:

$$
\epsilon_{\Omega, i}^{N_{1} N_{2}}=\epsilon_{\Omega, i}^{N_{1} N_{2}}\left(N_{i}\right)
$$

where the first subscript specifies that we are considering the locally-generated error, and the second subscript is the directional component. 
(e) The $\tau$-estimation procedure is performed element-wise while keeping the polynomial order in other elements sufficiently high so that

$$
\left\|\epsilon_{\partial \Omega}^{N}\right\| \ll\left\|\epsilon_{\Omega}^{N}\right\|
$$

This assumption follows from Remark 2.

As for (a) and (b), assumptions (c) and (d) also follow from the work of Rubio et al. [176, 177]. Notice that assumptions (a), (b), (c) and (d) are consistent with the dependence of the non-isolated truncation error on the discretization error (7.27). Furthermore, assumption (e) imposes an additional requirement for Proposition 1 to hold, namely that the polynomial orders of the elements not being analyzed must be maintained high enough during the $\tau$-estimation procedure so that the externallygenerated contributions to the discretization error are smaller than the internallygenerated ones.

Let us note that the assumption (d) implies that the discretization error in one direction does not change considerably for smooth solutions in the asymptotic range when the polynomial order in another direction is changed,

$$
\begin{aligned}
& \boldsymbol{\epsilon}_{j}^{N_{i} P_{j}} \approx \boldsymbol{\epsilon}_{j}^{P_{i} P_{j}}, \\
& \boldsymbol{\epsilon}_{i}^{N_{i} P_{j}} \neq \boldsymbol{\epsilon}_{i}^{P_{i} P_{j}},
\end{aligned}
$$

with $i \neq j$, and $1 \leq i, j \leq 2$.

As was seen in Section 7.3.5, the steady-state non-isolated truncation error in the DGSEM can be expressed for any basis function $\phi$ in an element e as

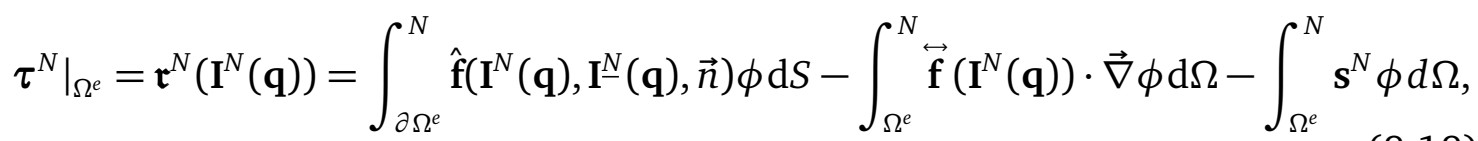

where the superindex " $e$ " has been dropped for the local solution. Since the DGSEM is a collocation method, the value computed with (8.18) corresponds to the non-isolated truncation error on the node of the basis function $\phi$.

By inserting the definition of the discretization error, $\mathbf{q}=\mathbf{q}^{N}+\boldsymbol{\epsilon}^{N}$ (7.9), and expanding the fluxes using Taylor series we obtain

$$
\left.\left.\tau^{N}\right|_{\Omega^{e}} \approx \int_{\partial \Omega^{e}}^{N} \frac{\partial \hat{\mathbf{f}}}{\partial \mathbf{q}}\right|_{\mathbf{q}^{N}, \mathbf{q}^{N}, \vec{n}} \boldsymbol{\epsilon}^{N} \phi \mathrm{d} S+\left.\int_{\partial \Omega^{e}}^{N} \frac{\partial \hat{\mathbf{f}}}{\partial \mathbf{q}^{-}}\right|_{\mathbf{q}^{N}, \mathbf{q}^{N}, \vec{n}} \boldsymbol{\epsilon}^{N} \phi \mathrm{d} S-\left.\int_{\Omega^{e}}^{N} \frac{\partial \overleftrightarrow{\mathbf{f}}}{\partial \mathbf{q}}\right|_{\mathbf{q}^{N}} \boldsymbol{\epsilon}^{N} \cdot \vec{\nabla} \phi \mathrm{d} \Omega
$$

where the interpolant of the discretization error is omitted for readability, $\mathbf{I}^{N}\left(\boldsymbol{\epsilon}^{N}\right) \leftarrow$ $\epsilon^{N}, \epsilon^{N}$ is the discretization error of the element $e, \epsilon^{N}$ is the discretization error of a neighbor element connected through the surface $\partial \Omega$, and the quadrature errors are assumed to be of a lower magnitude than the discretization error of the solution $\mathbf{q}^{N}$. Notice that, for the sake of readability, the symbol for the external polynomial orders is the same as of the internal ones, i.e. $N$, although they can be different. 
We now want to approximate the non-isolated truncation error through $\tau$-estimation. We part from the definition of the discretization error (7.9). Adding and subtracting the discrete solution on a higher order grid, $\mathbf{q}^{P}$, yields

$$
\begin{aligned}
& \boldsymbol{\epsilon}^{N}=\mathbf{q}-\mathbf{q}^{P}+\mathbf{q}^{P}-\mathbf{q}^{N} \\
& \boldsymbol{\epsilon}^{N}=\boldsymbol{\epsilon}^{P}+\mathbf{q}^{P}-\mathbf{q}^{N} .
\end{aligned}
$$

Reorganizing we have

$$
\mathbf{q}^{P}=\mathbf{q}^{N}+\epsilon^{N}-\epsilon^{P}
$$

Therefore, the $\tau$-estimation is

$$
\begin{aligned}
\left.\tau_{P}^{N}\right|_{\Omega^{e}}=\left.\mathfrak{r}\left(\mathbf{I}^{N}\left(\mathbf{q}^{P}\right)\right) \approx \int_{\partial \Omega^{e}}^{N} \frac{\partial \hat{\mathbf{f}}}{\partial \mathbf{q}}\right|_{\mathbf{q}^{N}, \mathbf{q}^{N}, \vec{n}}\left(\boldsymbol{\epsilon}^{N}-\boldsymbol{\epsilon}^{P}\right) \phi \mathrm{d} S \\
\quad+\left.\int_{\partial \Omega^{e}}^{N} \frac{\partial \hat{\mathbf{f}}}{\partial \mathbf{q}-}\right|_{\mathbf{q}^{N}, \mathbf{q}^{N}, \vec{n}}\left(\epsilon^{N}-\boldsymbol{\epsilon}^{P}\right) \phi \mathrm{d} S-\left.\int_{\Omega^{e}}^{N} \frac{\partial \overleftrightarrow{\mathbf{f}}}{\partial \mathbf{q}}\right|_{\mathbf{q}^{N}}\left(\boldsymbol{\epsilon}^{N}-\boldsymbol{\epsilon}^{P}\right) \cdot \vec{\nabla} \phi \mathrm{d} \Omega .
\end{aligned}
$$

Since (7.18) makes it possible to decouple the discretization error inside an element into a locally-generated and an externally-generated component, (8.21) can be rewritten as

$$
\begin{array}{r}
\left.\left.\tau_{P}^{N}\right|_{\Omega^{e}} \approx \int_{\partial \Omega^{e}}^{N} \frac{\partial \hat{\mathbf{f}}}{\partial \mathbf{q}}\right|_{\mathbf{q}^{N}, \mathbf{q}^{-}, \vec{n}}\left(\epsilon_{\Omega}^{N}-\epsilon_{\Omega}^{P}\right) \phi \mathrm{d} S-\left.\int_{\Omega^{e}}^{N} \frac{\partial \overleftrightarrow{\mathbf{f}}}{\partial \mathbf{q}}\right|_{\mathbf{q}^{N}}\left(\epsilon_{\Omega}^{N}-\epsilon_{\Omega}^{P}\right) \cdot \vec{\nabla} \phi \mathrm{d} \Omega \\
+\left.\int_{\partial \Omega^{e}}^{N} \frac{\partial \hat{\mathbf{f}}}{\partial \mathbf{q}}\right|_{\mathbf{q}^{N}, \mathbf{q}^{N}, \vec{n}}\left(\epsilon_{\partial \Omega}^{N}-\epsilon_{\partial \Omega}^{P}\right) \phi \mathrm{d} S-\left.\int_{\Omega^{e}}^{N} \frac{\partial \overleftrightarrow{\mathbf{f}}}{\partial \mathbf{q}}\right|_{\mathbf{q}^{N}}\left(\epsilon_{\partial \Omega}^{N}-\epsilon_{\partial \Omega}^{P}\right) \cdot \vec{\nabla} \phi \mathrm{d} \Omega \\
+\left.\int_{\partial \Omega^{e}}^{N} \frac{\partial \hat{\mathbf{f}}}{\partial \mathbf{q}^{-}}\right|_{\mathbf{q}^{N}, \mathbf{q}^{N}, \vec{n}}\left(\epsilon^{N}-\epsilon^{P}\right) \phi \mathrm{d} S .
\end{array}
$$

Equation (8.22) holds even for anisotropic discretizations, i.e. $N=\left(N_{1}, N_{2}, N_{3}\right)$ and $P=\left(P_{1}, P_{2}, P_{3}\right)$. Let us note that if the polynomial order of the elements that are not being analyzed is maintained as high as in the reference mesh, $\epsilon_{\partial \Omega}^{P}$ cancels out $\epsilon_{\partial \Omega}^{N}$ and $\boldsymbol{\epsilon}^{N}-\epsilon^{-} \approx 0$, i.e. the $\tau$-estimation provides the locally-generated truncation error.

Let us now consider the $2 \mathrm{D}$ anisotropic coarsening in the direction $i\left(\mathrm{~N}=\left(N_{i}, P_{j}\right)\right.$, $\left.\mathbf{P}=\left(P_{i}, P_{j}\right)\right)$. Taking into account assumptions $(c)$ and $(d)$, we obtain

$$
\begin{aligned}
\left.\tau_{\mathbf{P}}^{\mathrm{N}}\right|_{\Omega^{e}} \approx & \left.\int_{\partial \Omega^{e}}^{\mathbf{N}} \frac{\partial \hat{\mathbf{f}}}{\partial \mathbf{q}}\right|_{\mathbf{q}^{\mathrm{N}}, \mathbf{q}^{\mathrm{N}}, \vec{n}}\left(\epsilon_{\Omega, i}^{\mathbf{N}}-\epsilon_{\Omega, i}^{\mathbf{P}}\right) \phi \mathrm{d} S-\left.\int_{\Omega^{e}}^{\mathbf{N}} \frac{\partial \overleftrightarrow{\mathbf{f}}}{\partial \mathbf{q}}\right|_{\mathbf{q}^{\mathrm{N}}}\left(\epsilon_{\Omega, i}^{\mathbf{N}}-\epsilon_{\Omega, i}^{\mathbf{P}}\right) \cdot \vec{\nabla} \phi \mathrm{d} \Omega \\
+ & \left.\int_{\partial \Omega^{e}}^{\mathbf{N}} \frac{\partial \hat{\mathbf{f}}}{\partial \mathbf{q}}\right|_{\mathbf{q}^{\mathrm{N}}, \mathbf{q}^{\mathrm{N}, \vec{n}}}\left(\epsilon_{\partial \Omega}^{\mathbf{N}}-\epsilon_{\partial \Omega}^{\mathbf{P}}\right) \phi \mathrm{d} S-\left.\int_{\Omega^{e}}^{\mathbf{N}} \frac{\partial \overleftrightarrow{\mathbf{f}}}{\partial \mathbf{q}}\right|_{\mathbf{q}^{\mathbf{N}}}\left(\epsilon_{\partial \Omega}^{\mathbf{N}}-\epsilon_{\partial \Omega}^{\mathbf{P}}\right) \cdot \vec{\nabla} \phi \mathrm{d} \Omega \\
& +\left.\int_{\partial \Omega^{e}}^{\mathbf{N}} \frac{\partial \hat{\mathbf{f}}}{\partial \mathbf{q}-}\right|_{\mathbf{q}^{\mathrm{N}}, \mathbf{q}^{\mathrm{N}, \vec{n}}}\left(\epsilon^{-}-\epsilon^{-}\right) \phi \mathrm{d} S
\end{aligned}
$$


It is important to note that for sufficiently smooth solutions, the discretization errors on the high-order mesh of order $\mathbf{P}$ are smaller than the discretization errors on the mesh of order $\mathbf{N}$, and therefore can be neglected. Finally, if assumptions (c) and (e) hold, (8.23) implies that the anisotropic version of (8.22), where $N=\left(N_{1}, N_{2}, N_{3}\right)$, can be reconstructed by summing all the directional components (8.23) if the quadrature errors are neglected. I.e., we recover (8.4):

$$
\tau^{N_{1} N_{2}} \approx \tau_{P_{1} P_{2}}^{N_{1} P_{2}}+\tau_{P_{1} P_{2}}^{P_{1} N_{2}}
$$

In Chapter 5, the Jacobians of the viscous flux were evaluated with respect to $\mathbf{q}$ (with $\vec{\nabla} \mathbf{q}=$ constant) and $\vec{\nabla} \mathbf{q}$ (with $\mathbf{q}=$ constant). This enabled the evaluation of the Jacobians and the derivation of implicit discretizations. In this section, however, only the total Jacobians with respect to $\mathbf{q}$ are considered. This is a valid approach, even for viscous discretizations, since the Jacobians are only used for the proof and do not need to be evaluated for the error estimate.

\subsubsection{Convergence Behavior of the Anisotropic Truncation Error}

In this section we analyze the convergence properties of the truncation error map using Proposition 1. Let us first consider the directional components of the truncation error.

Proposition 2 The directional components of the locally-generated truncation error exhibit spectral convergence with respect to the polynomial order in the corresponding direction,

$$
\left\|\tau_{\Omega^{e}, i}\right\|_{\infty} \leq C_{i}^{e} e^{-N_{i}^{e} \eta_{i}^{e}} .
$$

Proof. 2 According to (8.2), the truncation error can be decoupled into directional components. If we insert (8.2) into (7.32) we obtain for $2 D$,

$$
\left\|\hat{\tau}_{\Omega^{e}, 1}^{N}+\hat{\tau}_{\Omega^{e}, 2}^{N}\right\|_{\infty} \leq C_{1}^{e} e^{-N_{1}^{e} \eta_{1}^{e}}+C_{2}^{e} e^{-N_{2}^{e} \eta_{2}^{e}} .
$$

Furthermore, according to (8.3), each directional component of the locally-generated truncation error, $\tau_{\Omega, i}$, depends solely on the polynomial order in the corresponding direction, $N_{i}$. Now, analyzing the dependencies term by term and considering that the constants $C_{1}$ and $C_{2}$ may be adjusted, we recover (8.25) for the isolated truncation error.

In the same way, if we insert (8.2) into (7.28), and take into account the dependencies (8.3), we recover (8.25) for the non-isolated truncation error.

Now, we are able to analyze the convergence behavior along lines of the truncation error map, where a line in $d$ dimensions is defined as,

$$
a_{1} N_{1}+a_{2} N_{2}+\cdots+a_{d} N_{d}=b
$$

with $a_{i}, b \in \mathbb{R}$. 
Proposition 3 The total truncation error does not necessarily decrease exponentially along lines of the truncation error map.

Proof. 3 The corresponding positive statement can be easily proven wrong with a counterexample. Let us consider a $2 D$ anisotropic discretization. From Proposition 2, we know that the truncation error of each directional component in an element, $\tau_{i}^{N}$, decreases exponentially when increasing $N_{i}$; and that the rate of decrease is $\eta_{i}$, a constant that depends on the smoothness of the solution in the direction $i$.

Let us suppose that for a certain element in a mesh, the directional components of the error have the same value for a specific combination of polynomial orders $\left(\bar{N}_{1}, \bar{N}_{2}\right)$ in the infinity norm,

$$
\left\|\tau_{1}^{\bar{N}_{1} \bar{N}_{2}}\right\|_{\infty}=\left\|\tau_{2}^{\bar{N}_{1} \bar{N}_{2}}\right\|_{\infty}=C .
$$

Let us analyze the convergence rate along an iso- $N_{i}$ line of the truncation error map with constant $N_{1}=\bar{N}_{1}$, i.e. the convergence rate of $\tau^{\bar{N}_{1} N_{2}}$ as a function of $N_{2}$. Note that, along that line we have, according to assumption (b),

$$
\begin{aligned}
\tau_{1}^{\bar{N}_{1} N_{2}} & \approx \tau_{1}^{\bar{N}_{1} \bar{N}_{2}} \\
\left\|\tau_{1}^{\bar{N}_{1} N_{2}}\right\|_{\infty} & \approx\left\|\tau_{1}^{\bar{N}_{1} \bar{N}_{2}}\right\|_{\infty}=C .
\end{aligned}
$$

Furthermore, assumption (a) states that the total truncation error along the line of the map is

$$
\tau^{\bar{N}_{1} N_{2}} \approx \tau_{1}^{\bar{N}_{1} N_{2}}+\tau_{2}^{\bar{N}_{1} N_{2}}
$$

Substituting (8.29) into (8.30) and using the triangle inequality yields:

$$
\left\|\tau^{\bar{N}_{1} N_{2}}\right\|_{\infty} \leq C+\left\|\tau_{2}^{\bar{N}_{1} N_{2}}\right\|_{\infty} .
$$

Remember that, according to Definition 8.1, the truncation error map is the dependence of $\log \left\|\tau^{N_{1} N_{2}}\right\|_{\infty}$ on $\left(N_{1}, N_{2}\right)$. Therefore, taking logarithms of both sides and rearranging, (8.31) can be rewritten in two equivalent forms:

$$
\begin{aligned}
& \text { i) } \quad \log \left\|\tau^{\bar{N}_{1} N_{2}}\right\|_{\infty} \leq \log \left\|\tau_{2}^{\bar{N}_{1} N_{2}}\right\|_{\infty}+\log \left(1+\frac{C}{\left\|\tau_{2}^{\bar{N}_{1} N_{2}}\right\|_{\infty}}\right), \\
& \text { ii) } \quad \log \left\|\tau^{\bar{N}_{1} N_{2}}\right\|_{\infty} \leq \log (C)+\log \left(1+\frac{\left\|\tau_{2}^{\bar{N}_{1} N_{2}}\right\|_{\infty}}{C}\right) .
\end{aligned}
$$

For $N_{2} \ll \bar{N}_{2}$, the second term on the right-hand side of (8.32) vanishes, which indicates that the convergence rate of the truncation error along an iso- $N_{i}$ line of constant $N_{1}=\bar{N}_{1}$ tends to $\eta_{2}$. On the other hand, for $N_{2} \gg \bar{N}_{2}$, the second term on the right-hand side of (8.33) vanishes, which implies that the convergence rate along an iso- $N_{i}$ line of constant $N_{1}=\bar{N}_{1}$ tends to zero, since the truncation error is bounded by $\log (C)$, a constant that does not depend on $N_{2}$. In other words, the iso- $N_{i}$ line on the hyperplane for $N_{1}=\bar{N}_{1}$ is not a straight line. 


\subsubsection{High-Order Extrapolation of the Truncation Error Estimations}

In this section, we show how to extrapolate the truncation error estimates (inner map) that can be obtained by applying Proposition 1. Since Proposition 3 rules out the possibility of extrapolating along iso- $N_{i}$ lines of the truncation error map, we take advantage of the anisotropic behavior of the truncation error in(8.2) and the spectral convergence of its directional components, Proposition 2. The proposed extrapolation procedure, which is valid for both the isolated and non-isolated truncation error, can be summarized in three steps:

1. Perform anisotropic coarsening to obtain $\tau_{i}$ using Proposition 1 and construct the inner truncation error map directly. In $d$ dimensions, this requires only $n_{\text {eval }}^{\text {new }}$ evaluations of the discrete partial differential operator $\mathfrak{R}^{N}$, where

$$
n_{\text {eval }}^{\text {new }}=\sum_{i=1}^{d}\left(P_{i}-1\right) \text {. }
$$

2. Compute $\log \left\|\tau_{i}\right\|_{\infty}$ and perform a linear regression analysis in the direction $i$ in order to describe the behavior of $\log \left\|\tau_{i}\right\|_{\infty}$ as a function of $N_{i}$. This is supported on the spectral behavior of the directional components of the truncation error proved in Proposition 2.

3. Construct the outer truncation error map using (8.4) and the extrapolated values of $\log \left\|\tau_{i}\right\|_{\infty}$,

$$
\log \|\tau\|_{\infty}=\log \left\|\sum_{i=1}^{d} \tau_{i}\right\|_{\infty} .
$$

We refer to this procedure as high-order extrapolation since it does not suppose that $\log \|\tau\|_{\infty}$ has a linear behavior. On the contrary, it only supposes (based on Proposition 2) that $\log \left\|\tau_{i}\right\|_{\infty}$ is linear. An example is provided in Section 8.4.

\subsubsection{Theoretical Comparison of the New Anisotropic $\tau$-Esti- mation with Previous Approaches}

Figure 8.3(a) illustrates the theoretically predicted behavior of the truncation error map that is obtained with the new anisotropic $\tau$-estimation method for a toy (illustrative) problem: The values of the inner map were arbitrarily chosen. As noted in the proof of Proposition 3, the truncation error firstly decays exponentially for low $N_{j \neq i}$ along an iso- $N_{i}$ line, and then tends asymptotically to a constant value for high $N_{j \neq i}$. As can be seen, contrary to the low-order extrapolation, the new anisotropic $\tau$-estimation does not assume that the truncation error map has a linear behavior. That is the reason why it is called high-order extrapolation. 
Figure 8.3(b) shows a comparison of the hyperplane behavior with the one obtained using the new anisotropic $\tau$-estimation method. As can be seen, the hyperplane tends to underpredict the truncation error for some combinations of polynomial orders as compared to the new truncation error estimator.

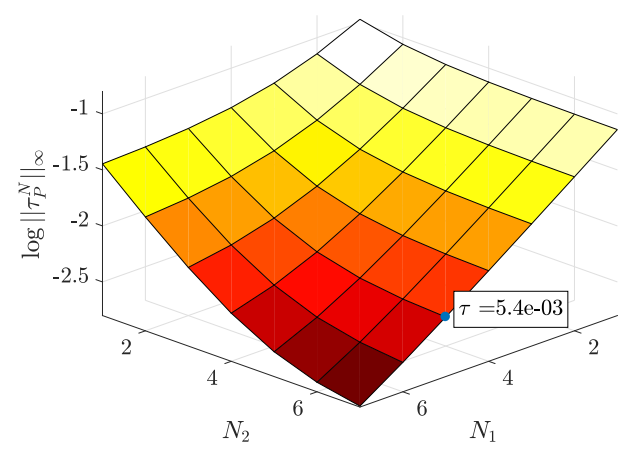

(a) Behavior predicted by the new anisotropic $\tau$-estimation method.

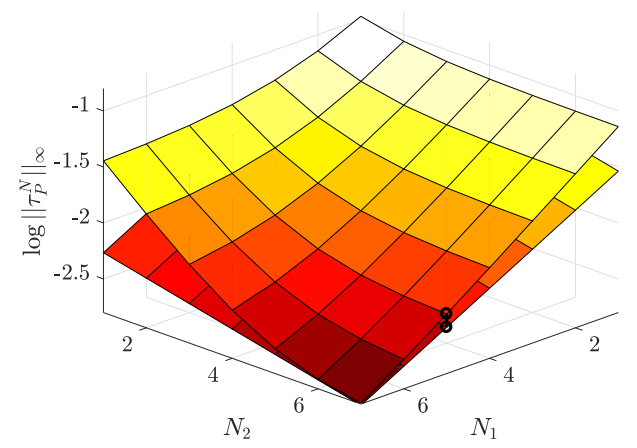

(b) Hyperplane behavior (Figure 8.2) overlapped with the values predicted by the new anisotropic $\tau$-estimation method

Figure 8.3: Spatial representation of two-dimensional anisotropic truncation error maps.

It is noteworthy that even though the method of Kompenhans et al. [110] supposes hyperplane behavior, their strategy to select the polynomial order in every direction independently (Step 4 of Section 8.2) minimizes the error of the low-order extrapolation (compare the values of $\|\tau\|_{\infty}$ in Figures 8.3(a) and 8.2). Furthermore, note that for $N_{1} \gg N_{2}$ and $N_{2} \gg N_{1}$, the new anisotropic estimation tends to have a hyperplane behavior. In fact, Kompenhans et al. [110, Section 5.1] state that only the values of the truncation error where $N_{1} \gg N_{2}$ or $N_{2} \gg N_{1}$ should be used for the least square fitting. The high-order extrapolation can be seen as a form of bypassing this requirement.

In summary, the main differences between the method of Kompenhans et al. with low-order extrapolation and the new p-anisotropic error estimator with highorder extrapolation are the following:

- The number of lower-order discretizations that are needed to generate the inner truncation error map (also, the number of evaluations of the discrete partial differential operator $-\mathfrak{R}^{N}$ ) in the method of Kompenhans et al. is

$$
n_{\text {eval }}=\prod_{i=1}^{d}\left(P_{i}-1\right),
$$

because the solution on the reference mesh must be injected into every single discretization of the inner map; whereas in the new $p$-anisotropic error 
estimator it is only

$$
n_{\text {eval }}^{\text {new }}=\sum_{i=1}^{d}\left(P_{i}-1\right),
$$

since only some lower-order discretizations are needed (8.4).

- The high-order extrapolation method, which uses the directional components of the truncation error, can provide more accurate estimates of the outer truncation error map for smooth solutions than the low-order extrapolation, which supposes a hyperplane behavior. The reason is that, while the high-order extrapolation relies on the spectral convergence of the directional components of the truncation error, which was proved in Proposition 2, Proposition 3 proved that the truncation error map does not have a hyperplane behavior.

- The price that must be paid for fewer evaluations of the discrete partial differential operator is a slightly decreased accuracy in the inner truncation error map when using the $p$-anisotropic error estimator proposed here. The reason is that the $\tau$-estimate in (8.8) and (8.21) contains a small error that depends on the discretization and/or interpolation errors on the reference mesh. When the truncation error is $\tau$-estimated for every single combination of polynomial orders (as in Kompenhans et al. [110]), this small error is only added once to the estimated value, whereas it is added once for each space direction when using the new $p$-anisotropic estimator.

\subsubsection{Additional Considerations}

As introduced in Section 2.2, the order of the numerical quadrature in the DGSEM is equal to the order of approximation. This improves the computational performance of the method but imposes a constraint on the geometry representation: The order of the mapping in every direction, $M_{i}$ in (2.33), must be at most the order of the DG approximation in that same direction, $M_{i} \leq N_{i}$, for the method to be free-stream (or constant state) preserving [112].

It is reasonable to think that the truncation error estimator presented here may fail if the mapping order on the reference mesh, $M^{P}$, is higher than the order of the DG discretizations that are needed to estimate the truncation error, i.e. if $M_{i}^{P}>N_{i}$. Namely, since the low-order discretizations must be constructed with an isoparametric mapping to be free-stream preserving, their geometry representation order is different than the one of the reference mesh. This causes an additional geometry representation error that is carried into in the truncation error estimate. Nevertheless, the truncation error estimator presented here is still consistent for $p$ adaptation purposes since the additional geometry representation error would also be present in the $p$-adapted meshes. See Section 8.4.4 for a demonstration of the validity of the truncation error estimate under high-order mappings. 
Finally, it is important to point out that the truncation error estimator presented in this chapter is not only useful for mesh adaptation purposes in the asymptotic regime, but may also be used outside of it. As has been pointed out before, the spectral convergence can only be expected when in the asymptotic regime. Therefore, if the solution is not smooth enough for the polynomial orders used or the selected mesh size, the errors are not expected to decrease exponentially fast when the polynomial order is increased. This is the case when shocks or any other kind of singularities are present in the solution. A low decay rate, a positive slope or a very low coefficient of determination in the linear regression, $R^{2}$, are signs that the analyzed element is not in the asymptotic regime.

When the solution inside an element is not in the asymptotic regime, it may be possible to bring the element into the asymptotic regime, or at least reduce the error considerably, by reducing its size. Namely, the size reduction can move the nonsmooth features to the element interface, or at least make the solution inside the element better behaved. The size reduction can be done by subdividing the elements via $h$-adaptation $[37,200]$, or by deforming the mesh via $r$-adaptation [20].

In this thesis, the analysis is restricted to $p$-adaptation methods. Therefore, meshes with appropriate element sizes for the specific applications are used, and in the uncommon case that the estimated truncation error does not exhibit a spectral decay, the maximum polynomial order is selected.

\subsection{Validation of the Anisotropic $\tau$-Estimation Method}

The compressible Navier-Stokes equations can be written in conservative form (see Appendix A) and discretized using the DGSEM, as explained in Section 2.2. To test the accuracy of the new $\tau$-estimation method presented in Section 8.3, a 2D manufactured solution test case is studied. The exact solution selected for the problem is

$$
\begin{aligned}
& \rho=p=e^{-5\left(4\left(x-\frac{1}{2}\right)^{2}+\left(y-\frac{1}{2}\right)^{2}\right)}+1, \\
& u=v=1,
\end{aligned}
$$

which is simulated in the unit square, as depicted in Figure 8.4.

By inserting (8.38) into the Navier-Stokes equations, the source term for the $2 \mathrm{D}$ compressible Navier-Stokes equations becomes

$$
\mathbf{s}=\left[\begin{array}{c}
s_{\rho} \\
s_{\rho u} \\
s_{\rho v} \\
s_{\rho e}
\end{array}\right]=\left[\begin{array}{c}
40\left(x-\frac{1}{2}\right)+10\left(y-\frac{1}{2}\right) \\
80\left(x-\frac{1}{2}\right)+10\left(y-\frac{1}{2}\right) \\
40\left(x-\frac{1}{2}\right)+20\left(y-\frac{1}{2}\right) \\
{\left[40\left(x-\frac{1}{2}\right)+10\left(y-\frac{1}{2}\right)\right]\left[\frac{1}{\gamma-1}+2\right]}
\end{array}\right] e^{5\left(4\left(x-\frac{1}{2}\right)^{2}+\left(y-\frac{1}{2}\right)^{2}\right)}
$$




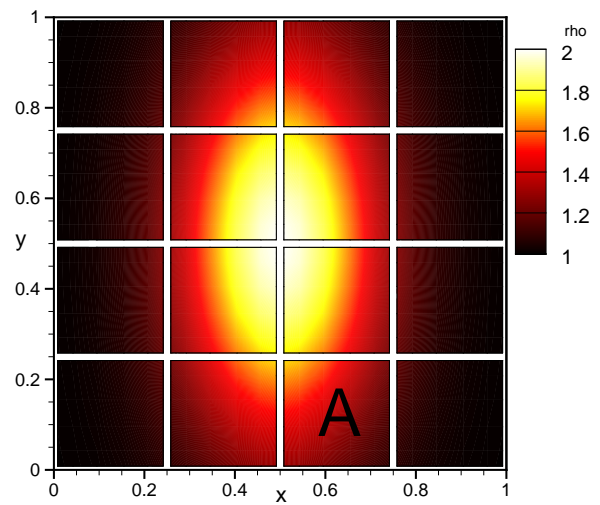

Figure 8.4: Density $(\rho)$ contours for the proposed manufactured solutions test case.

In Sections 8.4.1 and 8.4.2, we validate the new truncation error estimator and show that the estimates otained with it are more accurate than the ones obtained with the method of Kompenhans et al. [110] explained in Section 8.2. Since the method of Kompenhans et al. was formulated and used with the non-isolated truncation error, the results that are shown in Sections 8.4.1 and 8.4.2 were also obtained for the non-isolated truncation error estimator $\left(\tau_{P}^{N}\right)$. However, similar results can be obtained for the isolated truncation error.

In addition, in Section 8.4.3 we show that the isolated and non-isolated truncation error estimators perform similar when used to drive a $p$-adaptation procedure for the selected manufactured solution case.

Finally, Section 8.4.4 shows that the truncation error estimation generates accurate estimates under high-order mappings.

\subsubsection{Truncation Error Maps and Number of Degrees of Freedom}

In this section, we test the accuracy of the truncation error maps that are obtained with the new error estimator and the one developed by Kompenhans et al. [110] by comparing the estimates with the exact truncation error.

A fully time-converged solution $\left(\left\|\tilde{\mathfrak{R}}^{P}\right\|_{\infty}<10^{-10}\right)$ of order $5\left(P=P_{1}=P_{2}=5\right)$ is used to estimate the truncation error using the method of Section 8.3.1. The truncation error map that is estimated with the new method for element $A$ (marked in Figure 8.4) is depicted in Figure 8.5(a). Figure 8.5(b) shows the exact truncation error. It can be seen that the proposed method predicts a truncation error map that resembles the exact one, even for extrapolated values. These maps can be used to select an appropriate combination of polynomial orders so that a maximum truncation error threshold, $\tau_{\max }$, is achieved while employing a minimum number of degrees of freedom. 


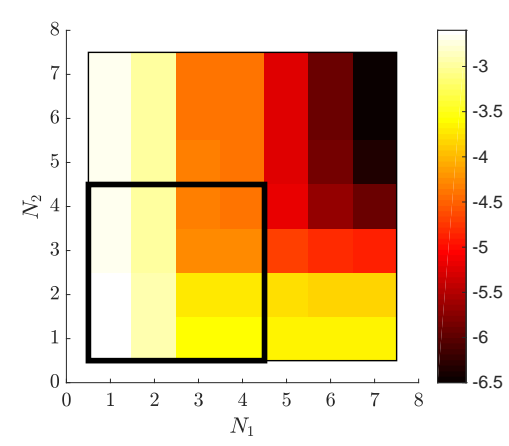

(a) Estimated $\left\|\tau_{5,5}^{N_{1} N_{2}}\right\|_{\infty}$ (the black box shows the limit between inner and outer maps).

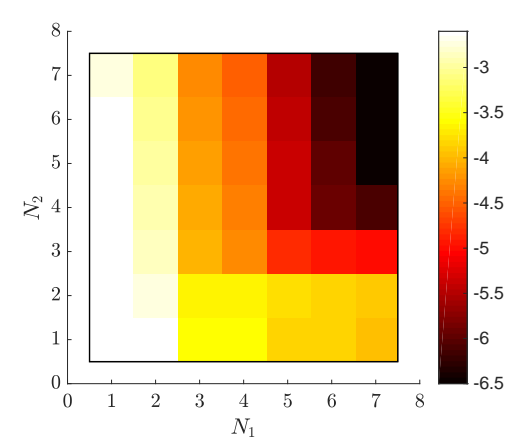

(b) Exact truncation error, $\left\|\tau^{N}\right\|_{\infty}$

Figure 8.5: Truncation error maps for element A (logarithmic scale).

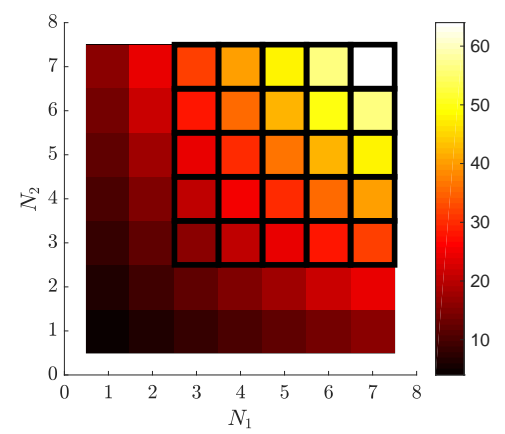

(a) $\tau_{\max }=10^{-4}$ (estimation).

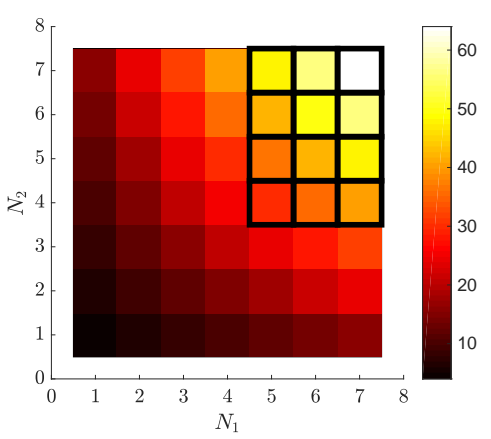

(c) $\tau_{\max }=10^{-5}$ (estimation).

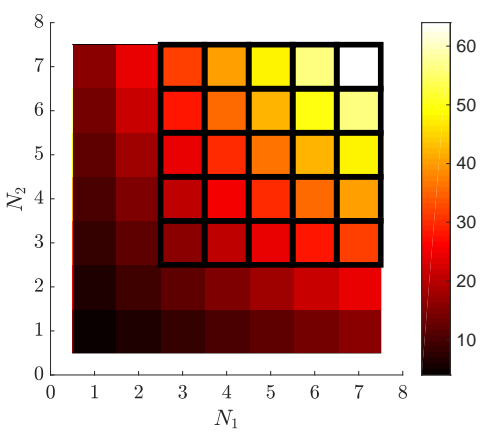

(b) $\tau_{\max }=10^{-4}$ (exact).

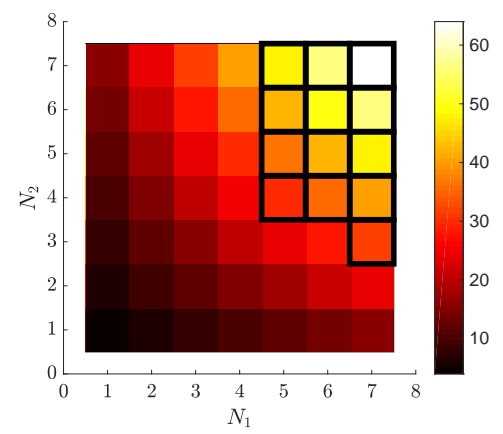

(d) $\tau_{\max }=10^{-5}$ (exact).

Figure 8.6: Contour of the number of degrees of freedom against the polynomial order. The combinations $\left(N_{1}, N_{2}\right)$ that fulfill the $\tau_{\max }$ threshold are marked with black squares. 
To select a combination of polynomial orders that fulfills a specific threshold, it is useful to take a look at the map of the number of degrees of freedom (NDOF). Figure 8.6 shows the map of NDOF for different $\left(N_{1}, N_{2}\right)$-combinations in the range $1 \leq N_{i} \leq 7$. The polynomial orders that achieve a specific truncation error $\|\tau\|_{\infty}<$ $\tau_{\max }$ (with $\tau_{\max }=10^{-4}$ and $\tau_{\max }=10^{-5}$ ) are marked with black squares. The estimated and the exact truncation error maps predict the exact same polynomial order combinations to fulfill the threshold $\tau_{\max }=10^{-4}$. However, for $\tau_{\max }=10^{-5}$, the estimated and exact maps differ in one predicted value. Let us remark that, although the results are not exactly the same for the estimated and exact truncation error maps, they both could be used for adaptation purposes. Notice that there are many alternatives that produce a truncation error in the desired range, but there is only one that minimizes the number of degrees of freedom and, therefore, the computational cost.

\subsubsection{Comparison with Previous Methods}

We now compare the output of the traditional and new truncation error estimators, together with the low-order and high-order extrapolation methods, respectively, against the exact truncation error for the manufactured solution problem.

Figure 8.7 shows the 3D representation of the exact truncation error map (a), the one obtained with the high-order extrapolation (b), and the one obtained with the low-order extrapolation (c) (here, we illustrate the complete hyperplane, although the method by Kompenhans et al. only constructs part of it), and an overlap of all the maps (d). The estimated maps were generated with the fully timeconverged solution of order $P_{1}=P_{2}=5$ that was used in last section. As can be seen, the truncation error map generated with the high-order extrapolation bears closer resemblance to the exact one than the hyperplane, which underpredicts the truncation error in some regions, as anticipated in Section 8.3.4.

The main drawback of the method of Kompenhans et al. [110] (Section 8.2) is the extrapolation. Figure 8.8(a) shows the 2D representation of the truncation error map that is generated with the method of Kompenhans et al. Note that, using this method, the full truncation error map is not generated but only the extrapolations for the iso- $N_{i}$ lines $N_{1}=P_{1}-1$ and $N_{2}=P_{2}-1$. Furthermore, we remark that although the method of Kompenhans et al. produces accurate results for $N_{i}<P_{i}$, it fails to predict the behavior of the truncation error for $N_{i} \geq P_{i}$, especially along the iso- $N_{1}$ line.

Figure 8.9(a) compares the truncation error estimations of the method of Kompenhans et al. and of the new method with the exact truncation error along the iso$N_{2}$ line that corresponds to a fixed value of $N_{2}=4$ (dotted lines of Figure 8.8(a)). It is remarkable that spectral convergence can be observed and both error estimators predict it.

The reason why the low-order extrapolation is able to predict spectral convergence along that iso- $N_{2}$ line can be drawn from Figure 8.9(b), which illustrates how 


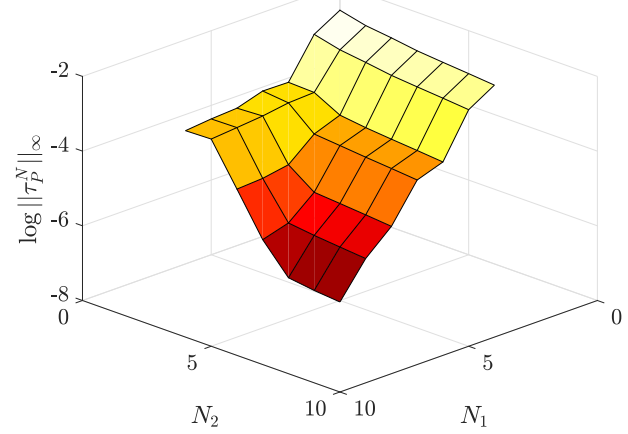

(a) Exact truncation error map

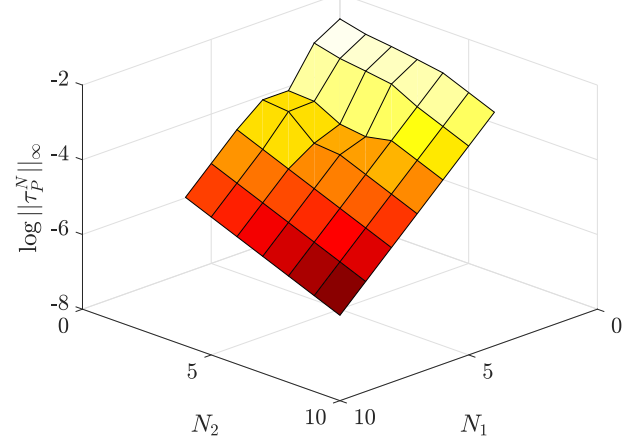

(c) Conventional $\tau$-estimation with loworder extrapolation (complete hyperplane)

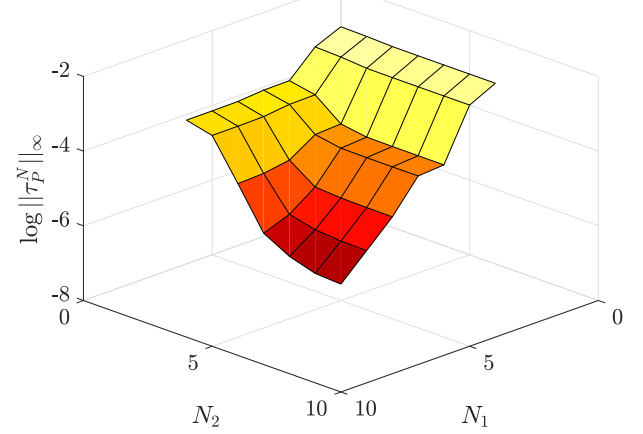

(b) New anisotropic $\tau$-estimation method with high-order extrapolation

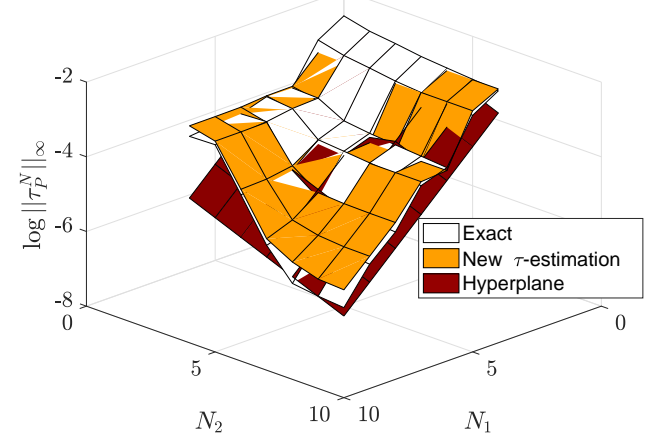

(d) Overlapped surfaces

Figure 8.7: Spatial representation of Two-dimensional anisotropic truncation error maps for the manufactured solutions test case. 


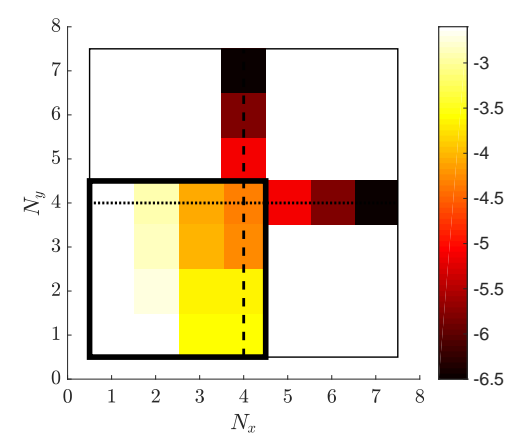

(a) Estimated $\left\|\tau_{5}^{N}\right\|_{\infty}$ using the method of Kompenhans et al. [110]. Outside the black box are the extrapolated values.

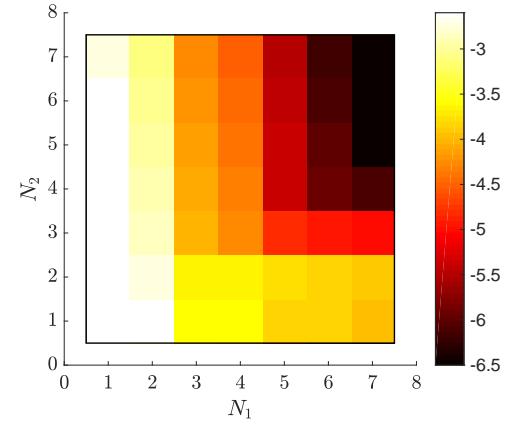

(b) Exact truncation error, $\left\|\tau^{N}\right\|_{\infty}$

Figure 8.8: Truncation error maps for element A (logarithmic scale).

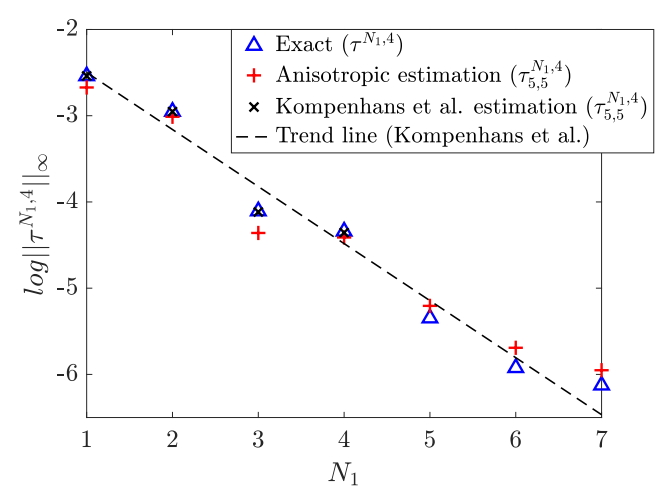

(a) Comparison with values obtained using (b) Estimated $\tau_{1}, \tau_{2}$ and $\tau_{5,5}^{N x, 4}$ with $P_{1}=P_{2}=$ the model of Kompenhans et al. [110].

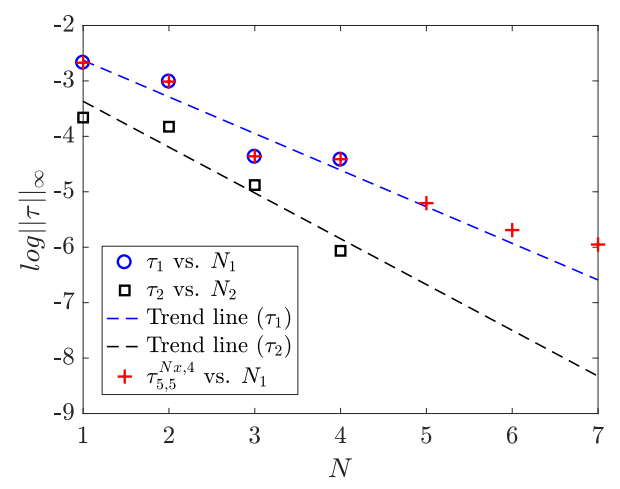

5 (proposed anisotropic model).

Figure 8.9: Truncation error estimation for $N_{2}=4$ in element A (dotted line of Figure 8.8).

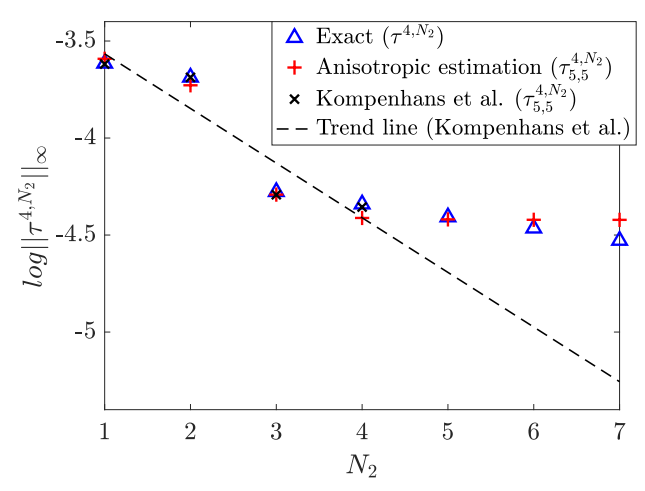

(a) Comparison with values obtained using (b) Estimated $\tau_{1}, \tau_{2}$ and $\tau_{5,5}^{4, N y}$ with $P_{1}=P_{2}=$ the model of Kompenhans et al. [110].

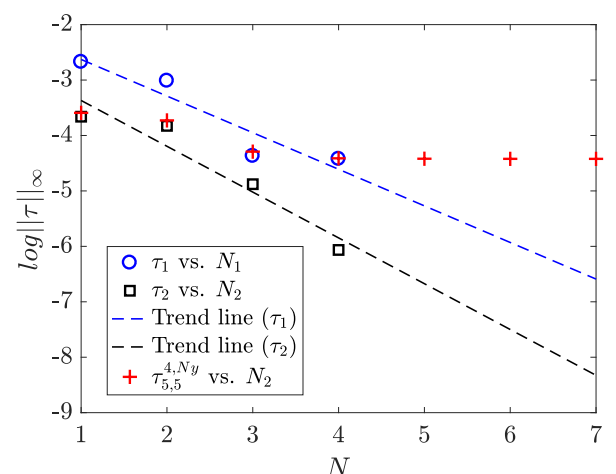

5 (proposed anisotropic model).

Figure 8.10: Truncation error estimation for $N_{1}=4$ in element A (dashed line of Figure 8.8). 
$\tau_{5,5}^{N_{1}, 4}$ is obtained using the new estimator of Section 8.3.1: The anisotropic contributions of the truncation error, $\tau_{1}$ and $\tau_{2}$, are used to generate independent trend lines and their values are then used to compute the total truncation error along the iso- $N_{2}$ line, $\tau_{5,5}^{N_{1}, 4}$. Spectral convergence is observed along the iso- $N_{2}=4$ line because the second component of the truncation error is very low,

$$
\left\|\tau_{2}\left(N_{2}=4\right)\right\|_{\infty} \approx 10^{-6}
$$

In fact, it is lower than all the values estimated for $\left\|\tau_{1}\right\|_{\infty}$. As a result, the total truncation error along the iso- $N_{2}=4$ line is mainly influenced by the first component. In other words, what we observe is actually the spectral convergence of $\tau_{1}$.

Let us now study the behavior of the truncation error for a fixed $N_{1}=4$ (dotted lines of Figure 8.8(a)). Figure 8.10(a) compares the truncation error estimates of the new method and the method of Kompenhans et al. with the exact truncation error. Let us note that the exact truncation error and the new estimate exhibit a stagnation behavior for $N_{2} \geq 3$, but the linear extrapolation of Kompenhans et al. under-predicts the truncation error for $N_{2}>4$.

To understand why the truncation error along the iso- $N_{1}$ line stagnates, Figure 8.10(b) illustrates how $\tau_{5,5}^{4, N_{2}}$ is obtained. Notice how, in this case, for $N_{2} \geq 3$ a stagnation in the decreasing rate of the truncation error occurs because

$$
\left\|\tau_{2}\left(N_{2} \geq 3\right)\right\|_{\infty} \leq\left\|\tau_{1}\left(N_{1}=4\right)\right\|_{\infty} .
$$

Therefore, the leading term of $\tau_{5,5}^{4, N_{2}}$ for $N_{2} \geq 4$ is $\left\|\tau_{1}\left(N_{1}=4\right)\right\|_{\infty}$

Spectral convergence can be expected for the decoupled terms $\left(\tau_{i}\right)$, but not necessarily for the total truncation error along iso- $N_{i}$ lines of the truncation error map (Propositions 2 and 3). In this particular case, when the assumptions of Remark 1 are violated, the error is underestimated in the outer truncation error map.

This simple example shows how the anisotropic error estimator formulated herein can produce more accurate estimates of the truncation error map for $N_{i} \geq P_{i}$ than previous estimators. Hence, in agreement with the results presented here, the assumptions of Section 8.3.1 are reasonable.

\subsection{3 $p$-Anisotropic Adaptation using the New Non-Isolated and Isolated Truncation Error Estimators}

As was discussed above, both the non-isolated and the isolated truncation error can be approximated using the anisotropic method introduced here. In this section, we study the exact truncation error of p-adapted discretizations obtained using the new error estimator for both the isolated and the non-isolated truncation error.

The fully converged solution of order $P_{1}=P_{2}=5$ is used as the reference mesh for the anisotropic $\tau$-estimation procedure with high-order extrapolation explained in Section 8.3.3. Truncation error thresholds are set as the target of the 
$p$-adaptation procedure in the range $10^{-7} \leq \tau_{\max }<10^{-1}$, and the polynomial order is selected after the estimation so that the number of degrees of freedom is minimized, which can be accomplished using the truncation error and NDOF maps as in Section 8.4.1). The maximum polynomial order allowed in any direction is set as $N_{\max }=10$, and the minimum polynomial order as $N_{\min }=1$.

Figure 8.11(a) shows the non-isolated truncation error that was achieved after the mesh adaptation as a function of the specified threshold, $\tau_{\max }$, and Figure 8.11(b) illustrates the isolated truncation error that was achieved for different values of $\tau_{\max }$. Two plateaus can be observed in both figures, one for $\tau_{\max } \leq 10^{-5}$ and one for $\tau_{\max } \geq 6 \times 10^{-3}$ as a consequence of the limiting polynomial orders. The first plateau corresponds to the minimum $\|\tau\|_{\infty}$ (and $\|\hat{\tau}\|_{\infty}$ ) that can be achieved when $N_{1}=N_{2}=N_{\max }=10$, and the second corresponds to the maximum $\|\tau\|_{\infty}$ (and $\|\hat{\tau}\|_{\infty}$ ) that can be achieved when $N_{1}=N_{2}=N_{\min }=1$ in every element. For the remaining specified thresholds, the truncation error achieved with both estimators is close to the specified value, the isolated truncation error being slightly better. The small gap between the ideal and achieved errors is attributed to the use of an estimate and not the exact truncation error.

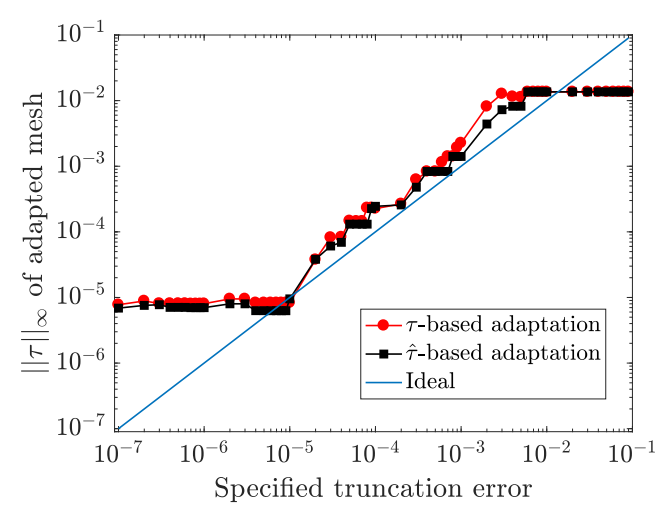

(a) Achieved non-isolated truncation error.

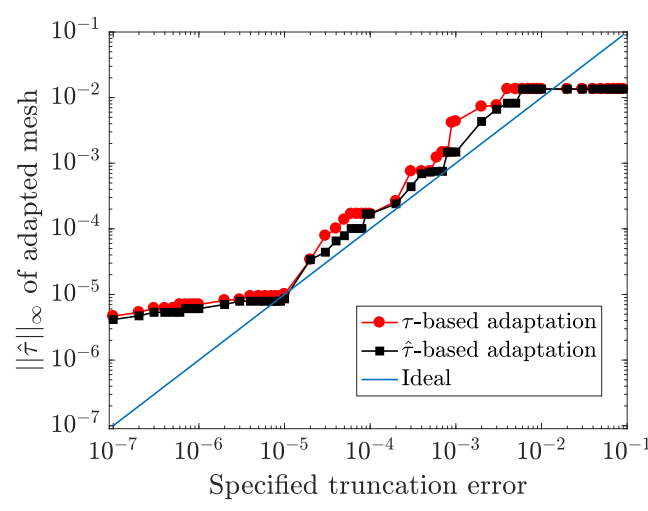

(b) b Achieved isolated truncation error.

Figure 8.11: Exact values of the achieved errors after the anisotropic $p$-adaptation based on non-isolated and isolated truncation error estimators with $N_{\min }=1$ and $N_{\max }=10$.

As these results show, controlling the isolated truncation error of a mesh also controls its non-isolated truncation error: a further advantage of the isolated estimator. In fact, we can write the non-isolated truncation error in terms of the isolated truncation error from the definitions in Sections 7.3 and 8.3.1:

$$
\tau^{N}=\hat{\tau}^{N}+\int_{\partial \Omega}^{N}\left(\hat{\mathbf{f}}\left(\mathbf{I}^{N}(\mathbf{q}), \mathbf{I}^{N}(\mathbf{q}), \vec{n}\right)-\overleftrightarrow{\mathbf{f}}\left(\mathbf{I}^{N}(\mathbf{q})\right) \cdot \vec{n}\right) \phi \mathrm{d} S
$$


Using the triangle inequality, (8.42) can be rewritten as

$$
\left\|\tau^{N}\right\|_{\infty}=\left\|\hat{\tau}^{N}\right\|_{\infty}+\left\|\int_{\partial \Omega}^{N}\left(\hat{\mathbf{f}}\left(\mathbf{I}^{N}(\mathbf{q}), \mathbf{I}^{N}(\mathbf{q}), \vec{n}\right)-\overleftrightarrow{\mathbf{f}}\left(\mathbf{I}^{N}(\mathbf{q})\right) \cdot \vec{n}\right) \phi \mathrm{d} S\right\|_{\infty} .
$$

Equation (8.43) suggests that the isolated truncation error is expected to control the non-isolated truncation error for sufficiently smooth solutions, for an appropriate choice of the numerical flux.

Taking into account that the main feature of the non-isolated truncation error is that it is affected by neighboring elements, we can conclude that the isolated estimator is a better driver for $p$-adaptation methods than the non-isolated truncation error estimator. Namely, because it would be excessively expensive to evaluate every possible combination of polynomial orders for each element of the mesh and its neighbors to feed the $p$-adaptation procedure.

\subsubsection{Anisotropic Error Estimation under High-Order Mappings}

In this section, the accuracy of the error estimator proposed in Section 8.3.3 is tested for elements with curved faces and high-order geometry mappings. For this test, the same manufactured solution is used with a mesh where all the element faces are sinusoidal functions (see Figure 8.12). Following (2.33), the referenceto-physical mapping can be approximated with polynomials of order $M_{i} \geq 1$, where $M_{1}=M_{2}=1$ means that the problem of Figure 8.4 is recovered. For higher values of $M_{i}$, the sinusoidal function is more accurately represented.

As in Section 8.4.1, a fully converged solution on a reference mesh of order $P_{1}=$ $P_{2}=5$ is used to approximate the truncation error using the new estimator for $1 \leq$ $N_{i} \leq 7$ The results are compared with the exact truncation error, which is computed by injecting the exact solution (8.38) into the polynomial order discretizations with $1 \leq N_{i} \leq 7$. Figure 8.13 shows the estimated and exact truncation errors for element A along two iso- $N_{i}$ lines, $N_{1}=4$ and $N_{2}=4$.

Let us now note that, to generate the truncation error map with the method of Section 8.3.3 and a reference solution of order $P_{1}=P_{2}=5$, it is necessary to generate eight additional low-order discretizations to estimate the error. Furthermore, as was discussed in Section 8.3.5, a DGSEM discretization must have $M_{i} \leq N_{i}$ to be free-stream preserving [112]. Therefore, for certain values of $M$, it is possible that the low-order discretizations require reduced order mappings.

For the sake of exposition, Table 8.1 shows the allowable mapping orders when the geometry is represented with a fourth order polynomial, $M_{1}=M_{2}=4$. As can be seen, only two of the lower-order discretizations $((5,4)$ and $(4,5))$ can retain the specified high-order mapping. This could be regarded as a limitation, but, as we will show, it does not pose a problem for the truncation error estimation. 


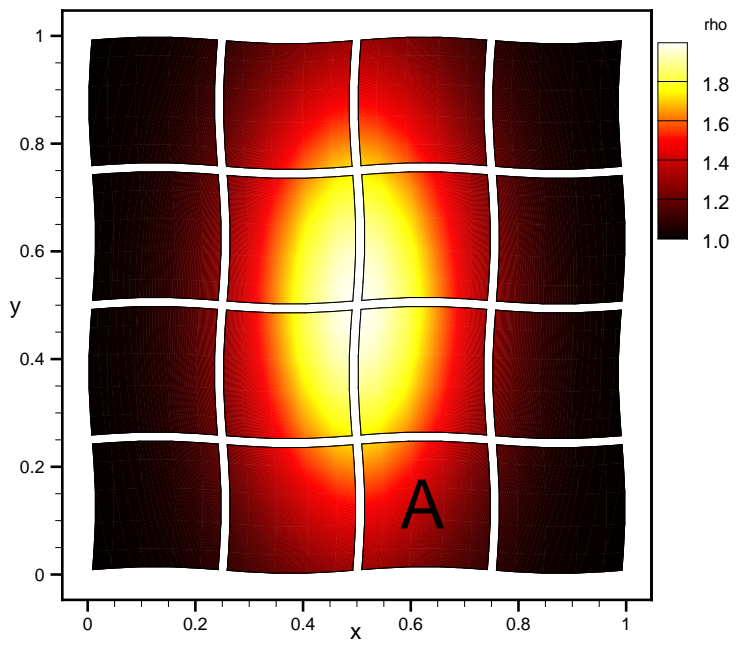

Figure 8.12: Density $(\rho)$ contours for the proposed manufactured solutions test case with curved boundaries.

Table 8.1: Allowable mapping orders for all the discretizations needed in an anisotropic error estimation. $P_{1}=P_{2}=5$ and $M_{1}=M_{2}=4$.

\begin{tabular}{l|ccccccccc}
\hline $\begin{array}{l}\text { Discretization } \\
\left(N_{1}, N_{2}\right)\end{array}$ & $(5,5)$ & $(5,4)$ & $(5,3)$ & $(5,2)$ & $(5,1)$ & $(4,5)$ & $(3,5)$ & $(2,5)$ & $(1,5)$ \\
\hline$M_{1}$ & 4 & 4 & 4 & 4 & 4 & 4 & 3 & 2 & 1 \\
$M_{2}$ & 4 & 4 & 3 & 2 & 1 & 4 & 4 & 4 & 4 \\
\hline
\end{tabular}

Two important conclusions can be drawn from Figure 8.13. First, the truncation error is higher for higher-order mappings (compare with Figures 8.9 and 8.10). This is consistent with the results reported by Botti [27], where the convergence deteriorates when the mapping order is increased. For this particular element, it can be seen that the $y$-component of the truncation error, $\tau_{2}$, is especially dependent on the mapping order for element A. Second, it can be seen that the computed error estimate is close to the exact truncation error for the different mapping orders in spite of the apparent limitation shown in Table 8.1. In fact, a new stagnation in the truncation error is successfully predicted for $N_{1} \geq 5$ when $M \geq 4$. As mentioned in Section 8.3.5, the extra geometry representation error contained in the lower-order discretizations is also present in the meshes where the exact truncation error is evaluated.

The accuracy of the DGSEM deteriorates when the mapping order is increased because no over-integration is performed. However, our estimation method still predicts the truncation error (which includes geometrically induced errors) accurately. 


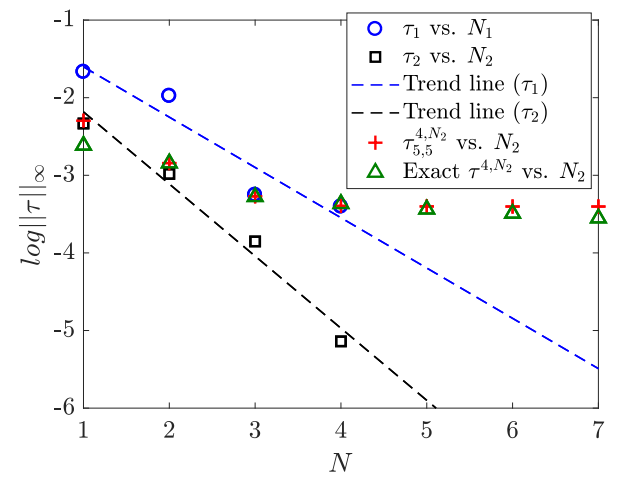

(a) $\tau_{5,5}^{4, N_{2}}$ and $\tau^{4, N_{2}}$ for $M=2$.

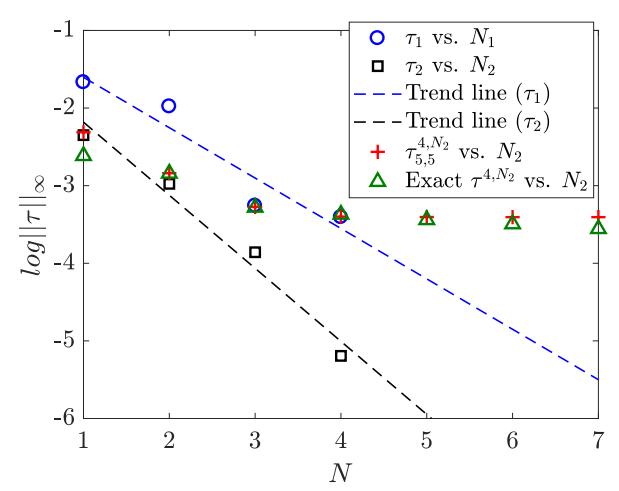

(c) $\tau_{5,5}^{4, N_{2}}$ and $\tau^{4, N_{2}}$ for $M=3$.

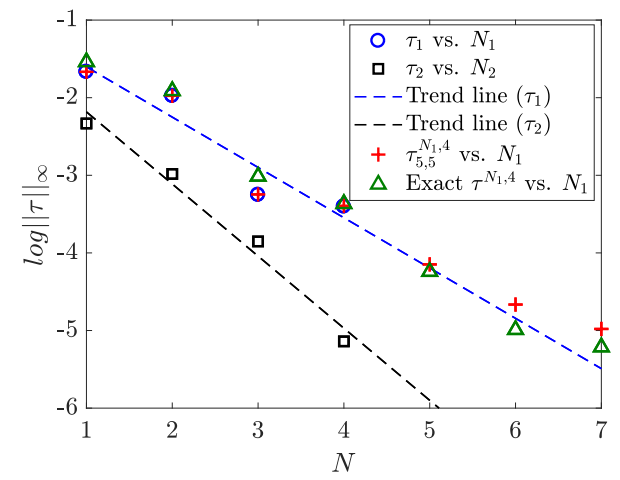

(b) $\tau_{5,5}^{N_{1}, 4}$ and $\tau^{N_{2}, 4}$ for $M=2$.

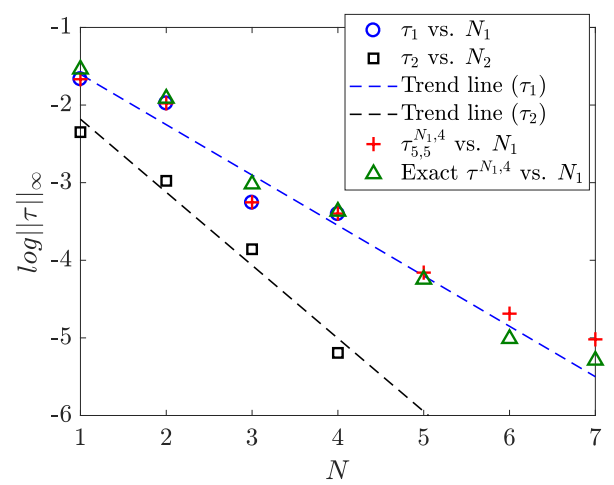

(d) $\tau_{5,5}^{N_{1}, 4}$ and $\tau^{N_{2}, 4}$ for $M=3$.

Figure 8.13: Anisotropic truncation error estimation (obtained with a reference solution of order $P_{1}=P_{2}=5$ ) under high-order mappings and comparison with the exact truncation error in element A for fixed $N_{1}=4(\mathrm{a}, \mathrm{c}, \mathrm{e}, \mathrm{g})$ and $N_{2}=4(\mathrm{~b}, \mathrm{~d}, \mathrm{f}, \mathrm{h})$ logarithmic scale. 


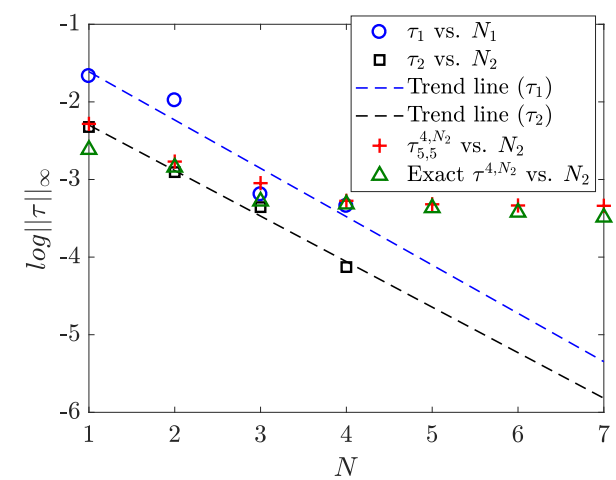

(e) $\tau_{5,5}^{4, N_{2}}$ and $\tau^{4, N_{2}}$ for $M=4$.

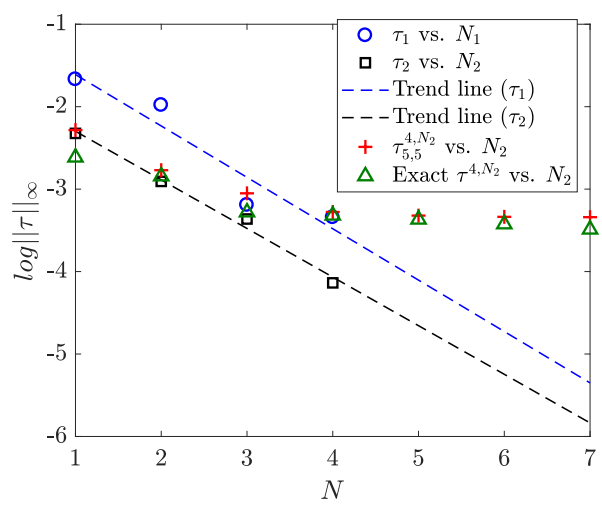

(g) $\tau_{5,5}^{4, N_{2}}$ and $\tau^{4, N_{2}}$ for $M=5$.

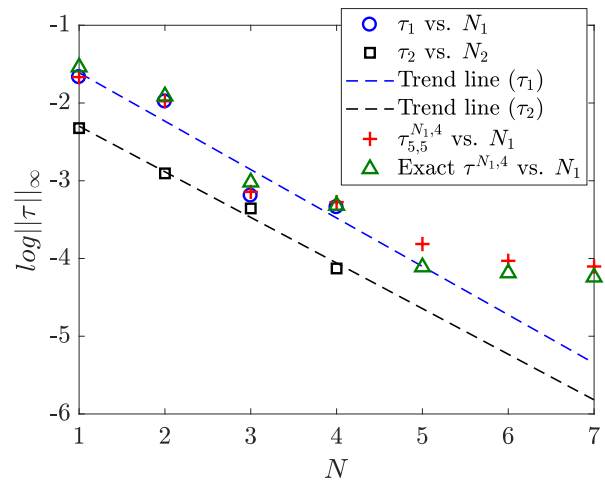

(f) $\tau_{5,5}^{N_{1}, 4}$ and $\tau^{N_{2}, 4}$ for $M=4$.

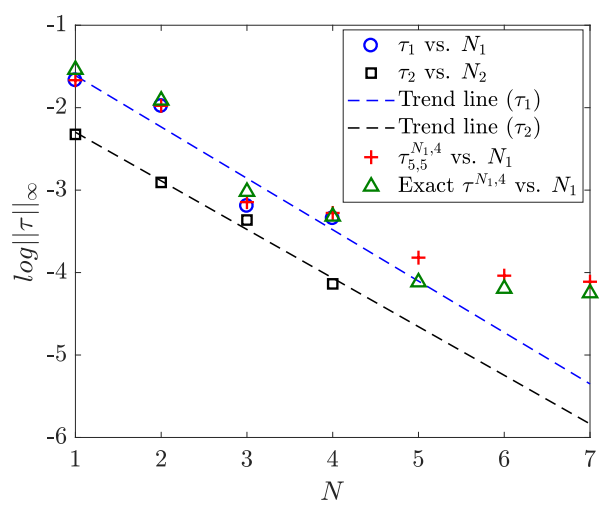

(h) $\tau_{5,5}^{N_{1}, 4}$ and $\tau^{N_{2}, 4}$ for $M=5$.

Figure 8.13: Anisotropic truncation error estimation (obtained with a reference solution of order $P_{1}=P_{2}=5$ ) under high-order mappings and comparison with the exact truncation error in element A for fixed $N_{1}=4$ (a,c,e,g) and $N_{2}=4$ (b,d,f,h) logarithmic scale. - Continued 


\subsection{Final Remarks}

In this chapter, a study of anisotropic truncation error estimators for the DGSEM, their convergence properties and accuracy was presented. The most important results are:

1. A new technique to evaluate the truncation error was developed, which is computationally more efficient than previous implementations: It requires a fewer number of floating-point operations and less memory. Furthermore, this technique extrapolates the truncation error better than previous methods. This allows one to use coarser reference meshes, hence improving the computational efficiency.

2. The new method provides truncation error estimations that are accurate enough to perform $p$-adaptation, as shown in Sections 8.4.1 and 8.4.3.

3. The isolated truncation error is better suited to drive a $p$-adaptation procedure than its non-isolated counterpart because the latter is affected by the discretization error in other regions. Also, as stated in Remark 2, the nonisolated truncation error estimator requires that the polynomial orders in the mesh are kept sufficiently high for the estimation procedure to work well. This translates into a more expensive $\tau$-estimation.

4. The method of Kompenhans et al. [110], in which every combination of $N=$ $\left(N_{1}, N_{2}, \cdots, N_{d}\right)$ is directly evaluated to generate the truncation error map, produces a slightly better estimate of the truncation error for $N_{i}<P_{i}$ than the error estimator proposed here, but fails to predict the truncation error for $N_{i} \geq P_{i}$ accurately. A good compromise could be to generate the truncation error map for $N_{i}<P$ using the method of Kompenhans et al., but then use the fully decoupled method to generate the extrapolated map. However, this strategy requires more storage and computational power. 

$\Gamma_{\text {Chapter }} 9$

\section{Steady $p$-Adaptation Using Truncation Error Estimates}

\section{Contents}

9.1 Introduction . . . . . . . . . . . . . . . . . . 143

9.2 Coupling the $p$-Anisotropic $\tau$-Estimation with Multigrid . . . 144

9.2 .1 Anisotropic Multigrid . . . . . . . . . . . . . . . . . . . 145

9.2.2 Uniform Coarsening versus High-Order Coarsening . . . . 146

9.3 The $p$-Adaptation Algorithm: Computing the New Polynomial Orders ....................... 148

9.4 Multi-Stage Adaptation Process . . . . . . . . . . . . . . . . 149

9.5 Numerical Results . . . . . . . . . . . . . . . . . . . 150

9.5.1 2D Flow over a Flat Plate . . . . . . . . . . . . . 150

9.5.2 3D Flow Around a Sphere . . . . . . . . . . . . . 157

9.6 Final Remarks . . . . . . . . . . . . . . . . . . . . . . 164

\subsection{Introduction}

In this chapter, we develop an efficient, anisotropic $p$-adaptation method for steadystate problems, which uses the new truncation error estimator that was developed in Chapter 8. This work has been reported by the author in $[178,179]$. As in the rest of this thesis, the DGSEM is used; however, the ideas presented here can be extended to any other DG method that uses tensor-product basis functions. We are interested in a low-cost estimation of the error, so we use the quasi a-priori $\tau$-estimation without correction of Section 7.4. 
In Section 9.2, it is shown that the anisotropic error estimation of Chapter 8 can be performed inside the multigrid cycle with negligible extra cost. As a consequence, it is possible to combine the error estimatod with the multigrid cycling strategy in a new anisotropic $p$-adaptation multigrid algorithm for steady-state problems that uses multigrid both as a solver and as an anisotropic error estimator. We describe how the coupling of multigrid and $p$-adaptation produces a speed-up in the computation of the solution.

At this point, we already have two of the three key tools to perform $p$-adaptation: an error estimator and a method to couple $p$-no-conforming elements (the mortar spaces of Section 7.5). The third key tool, an algorithm that computes the polynomial orders from the outcome of the error estimation, is detailed in Section 9.3.

Additionally, the coupling of multigrid and $p$-adaptation also enables us to propose a multi-stage adaptation process that uses a Full Multigrid Method (FMG) with increasing order reference solutions, which reduces the computational cost when very accurate results are required. That alternative to the original singlestage algorithm is presented in Section 9.4.

Finally, in Section 9.5, the $\tau$-based anisotropic $p$-adaptation is tested for the compressible Navier-Stokes equations, where we investigate two steady-state cases. First, the 2D boundary layer flow on a flat plate is used to assess the accuracy and computational cost of the algorithm, where a speed-up of 816 is achieved compared to the traditional explicit method without adaptation for the same error. Second, the 3D flow around a sphere is simulated and used to test the anisotropic properties of the $p$-adaptation method, where a speed-up of 152 is achieved compared to the traditional explicit method without adaptation for the same accuracy. The new multi-stage procedure achieved a speed-up of 2.6 in comparison to the single-stage method in highly accurate simulations.

\subsection{Coupling the $p$-Anisotropic $\tau$-Estimation with Multigrid}

In this section, we present a new technique to obtain steady-state solutions by coupling anisotropic $p$-adaptation methods and multigrid. As pointed out firstly by Brandt [33], and then recently used by Syrakos et al. [191] in the context of $h$-refinement techniques, the concept of truncation error arises naturally in nonlinear multigrid methods, also known as Full Approximation Scheme (FAS) multigrid methods (see Section 4.2.2). In fact, the first term of the non-isolated truncation error estimator (7.38),

$$
\mathscr{T}_{P}^{N}=\mathbf{H}^{N}\left(\underline{\mathbf{I}}_{P}^{N} \tilde{\mathbf{Q}}^{P}\right)-\underline{\mathbf{M}}^{N} \mathbf{S}^{N},
$$

is contained in the coarse-grid source term of the nonlinear multigrid scheme (4.13),

$$
\mathbf{S}^{N}=\underline{\mathbf{M}}^{-1} \mathbf{H}^{N}\left(\underline{\mathbf{I}}_{P}^{N} \tilde{\mathbf{Q}}^{P}\right)-\underline{\mathbf{I}}_{P}^{N} \tilde{\mathfrak{R}}^{P}
$$


Consequently, computing $\tau_{P}^{N}$ inside the multigrid cycle only involves a few additional operations. For the isolated truncation error, the operator $\hat{\mathbf{H}}^{N}$ must also be evaluated.

Two main differences with the traditional $\tau$-estimator, which directly injects the high-order solution into the coarser levels, can be identified. First, instead of interpolating the finest solution directly to every coarser discretization, the solution is interpolated level by level in multigrid methods. Second, the smoothing procedure modifies the finest solution before it is transferred to lower orders. In that regard, the error estimation is not expected to be affected negatively because the coarsegrid smoothing works on the fine-grid problem. As a consequence, the reference solution is expected to be improved after the smoothing sweeps. In fact, preliminary tests showed no significant difference between the multigrid $\tau$-estimations and the conventional ones.

In traditional $p$-multigrid techniques, the coarsening is usually performed in all coordinate directions simultaneously, i.e. the polynomial order of every local coordinate direction is decreased (isotropic multigrid). Therefore, only certain combinations of low-order polynomial orders are evaluated. This makes impossible to generate the full anisotropic truncation error map that is needed to perform anisotropic $p$-adaptation with the conventional $\tau$-estimation procedure (7.43),

$$
\tau_{P_{1} P_{2} P_{3}}^{N_{1} N_{2} N_{3}}=\mathfrak{r}^{N_{1} N_{2} N_{3}}\left(\mathbf{q}^{P_{1} P_{2} P_{3}}\right) .
$$

To be able to generate the full anisotropic truncation error map, a $p$-anisotropic multigrid procedure is proposed in Section 9.2.1.

\subsubsection{Anisotropic Multigrid}

In this section, we propose the use of an anisotropic multigrid method that works as both solver and $\tau$-estimator.

The classical approach to implement a $p$-multigrid method is to perform coarsening in all reference coordinate directions simultaneously. This strategy is referred to as isotropic multigrid. The anisotropic multigrid method that is presented here performs the coarsening in each reference coordinate direction at a time. In a 3D $p$-anisotropic multigrid method, when coarsening in $\xi$, the coarse grid problem is derived from (4.11) as

$$
\mathbf{R}^{N_{1} P_{2} P_{3}}\left(\mathbf{Q}^{N_{1} P_{2} P_{3}}\right)=\mathbf{S}^{N_{1} P_{2} P_{3}},
$$

where the source term is obtained from (4.13):

$$
\mathbf{S}^{N_{1} P_{2} P_{3}}=\underline{\mathbf{M}}^{-1} \mathbf{H}^{N_{1} P_{2} P_{3}}\left(\underline{\mathbf{I}}_{P_{1} P_{2} P_{3}}^{N_{1} P_{2} P_{3}} \tilde{\mathbf{Q}}^{P_{1} P_{2} P_{3}}\right)+\underline{\mathbf{I}}_{P_{1} P_{2} P_{3}}^{N_{1} P_{2} P_{3}} \mathfrak{R}^{P_{1} P_{2} P_{3}} .
$$

It is now evident that, in the same way as with the $p$-isotropic scheme, the first term of (9.5) requires the computation of a directional component of the truncation error,

$$
\mathscr{T}_{P_{1} P_{2} P_{3}}^{N_{1} P_{2} P_{3}}=\mathbf{H}^{N_{1} P_{2} P_{3}}\left(\underline{\mathbf{I}}_{P_{1} P_{2} P_{3}}^{N_{1} P_{2} P_{3}} \tilde{\mathbf{Q}}^{P_{1} P_{2} P_{3}}\right)-\underline{\mathbf{M}}^{N_{1} P_{2} P_{3}} \mathbf{S}^{N_{1} P_{2} P_{3}} .
$$


The new anisotropic truncation error estimator of Chapter 8 is recovered by summing all the directional components in (8.4):

$$
\mathscr{T}^{N_{1} N_{2} N_{3}} \approx \mathscr{T}_{1}^{N_{1} N_{2} N_{3}}+\mathscr{T}_{2}^{N_{1} N_{2} N_{3}}+\mathscr{T}_{3}^{N_{1} N_{2} N_{3}} \approx \mathscr{T}_{P_{1} P_{2} P_{3}}^{N_{1} P_{2} P_{3}}+\mathscr{T}_{P_{1} P_{2} P_{3}}^{P_{1} N_{2} P_{3}}+\mathscr{T}_{P_{1} P_{2} P_{3}}^{P_{1} P_{2} N_{3}} .
$$

It is noteworthy that both the isotropic and the anisotropic $p$-multigrid methods can be applied to $p$-isotropic and $p$-anisotropic discretizations interchangeably. If the anisotropic $p$-multigrid method is used to solve a $p$-isotropic discretization, the number of multigrid levels is the same in all coordinate directions, $N_{M G, i}=N_{M G, j}$. However, if the anisotropic $p$-multigrid method is used to solve a $p$-anisotropic discretization, the number of multigrid levels can be different in every coordinate direction, $N_{M G, i} \neq N_{M G, j}$.

To generate the truncation error estimation for all polynomial orders $N_{i}<P_{i}$, the V-cycling strategy of Section 4.2.2.1 is used. Figure 9.1 depicts the anisotropic 3V FAS cycle, where the coarsening is performed in one of the coordinate directions of the reference element in each V-cycle, and the directional component of the truncation error is estimated. In other words, instead of evaluating every possible combination of $N=\left(N_{1}, N_{2}, N_{3}\right)$ for $N_{i}<P_{i}$ (which can be a large number for 3D problems), as in $[110,176]$, the full truncation error map is constructed from the directional estimations of the anisotropic multigrid. One important advantage of using multigrid is that all the storage needed for the directional error estimators is already allocated in the anisotropic multigrid routines and only a few inexpensive additional computations are required.

In a general 3D mesh, the reference coordinates of an element, $(\xi, \eta, \zeta)$, are commonly not aligned with the reference coordinates of neighbor elements. This can pose a problem for the non-isolated truncation error estimator if an anisotropic $3 \mathrm{~V}$ FAS cycle is used, where all the elements of a mesh are coarsened simultaneously on the same reference coordinate direction. Namely, approximation errors can be advected and diffused into an element during the coarsening procedure in an amount that is difficult to quantify. On the other hand, the lack of alignment does not affect the isolated truncation error estimator as it neglects the contribution of the neighboring elements.

In this work, we want to generate coarse-grid discretizations with $\Delta N=1$ for two reasons. First, because $\Delta N=1$ ensures that the smoothing is acting in all possible frequencies, as explained in Section 4.2.2. Second, because it is desirable to have a truncation error estimate for every $N_{i}<P_{i}$ so that as many points are available for the $\tau$-extrapolation.

\subsubsection{Uniform Coarsening versus High-Order Coarsening}

We propose two ways to obtain the polynomial orders of the coarser discretizations. Let us define the tensor $\mathscr{N}$, which contains the polynomial orders in all reference frame directions of all the elements in a mesh,

$$
\mathscr{N}=\left(N^{1}, N^{2}, \cdots, N^{e}, \cdots, N^{K}\right)
$$




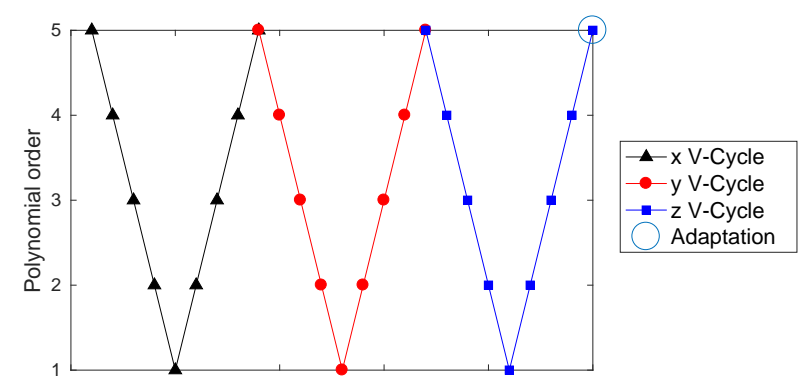

Figure 9.1: Adaptation process: Anisotropic 3V FAS cycle and subsequent adaptation.

where $K$ is the number of elements of the mesh, $e$ is the element index, and $N^{e}=$ $\left(N_{1}^{e}, N_{2}^{e}, N_{3}^{e}\right)$ are the polynomial orders of each element.

After the $p$-adaptation procedure is completed, the mesh has elements with non-uniform anisotropic polynomial orders. For $\Delta N=1$, the number of multigrid levels is $N_{M G}=\max (\mathscr{N})-N_{\text {coarse }}+1$ for the isotropic multigrid and $N_{M G, i}=\max \left(\mathscr{N}_{i}\right)-$ $N_{\text {coarse }}+1$ for each reference direction in the anisotropic multigrid (the latter is a function of the maximum polynomial order per coordinate direction). Let us define two ways to perform the coarsening inside a multigrid cycle:

- Uniform coarsening: The coarsening is performed in all elements simultaneously,

$$
\left(N_{i}^{e}\right)_{\text {level }}=\left(N_{i}^{e}\right)_{\text {level }+1}-\Delta N,
$$

except in the elements where the minimum polynomial order allowed has been reached:

$$
\text { if }\left(\left(N_{i}^{e}\right)_{\text {level }}<N_{\text {coarse }}\right) \text { then }\left(N_{i}^{e}\right)_{\text {level }}=N_{\text {coarse }} \text {. }
$$

- High-order coarsening: Since the maximum polynomial order in every multigrid level can be known beforehand,

$$
\left(N_{i}\right)_{\text {level }}^{\max }=\max \left(\mathscr{N}_{i}\right)-\Delta N\left(N_{M G, i}-\text { level }\right),
$$

we can coarsen only the elements that do not fulfill this condition:

$$
\text { if }\left(\left(N_{i}^{e}\right)_{\text {level }+1}>\left(N_{i}\right)_{\text {level }}^{\max }\right) \text { then }\left(N_{i}^{e}\right)_{\text {level }}=\left(N_{i}\right)_{\text {level }}^{\max } \text {. }
$$

In this way only the high-order elements are coarsened. Here, we use $N_{\text {coarse }}=$ $\Delta N=1$. Therefore, (9.11) reduces to

$$
\left(N_{i}\right)_{\text {level }}^{\max }=\text { level }
$$


Notice that equations (9.9) to (9.12) are valid for isotropic and anisotropic multigrid procedures. In the former, $N_{M G, i}$ must be simply replaced by $N_{M G}$, $\max \left(\mathscr{N}_{i}\right)$ by $\max (\mathscr{N})$, and the operations are performed in all coordinate directions. In the latter, the operations are only performed in the coordinate direction in which the coarsening is done. Furthermore, both coarsening methods are equivalent for isotropic polynomial discretizations.

In this work, only high-order coarsening is used. One could argue that the uniform coarsening involves less computational cost than the high-order coarsening since coarse discretizations have fewer degrees of freedom. Nevertheless, the latter has two main advantages:

- Several preliminary tests showed that uniform coarsening is more sensitive to the time-step size than high-order coarsening for highly anisotropic meshes in $2 \mathrm{D}$ and $3 \mathrm{D}$.

- In 3D meshes that are not 2D extrusions, $p$-nonconforming discretizations require the mapping order to be $M_{i} \leq N_{i} / 2$, as noted in Section 2.2. This means that the minimum polynomial order of the mesh must be $\min (\mathscr{N}) \geq 2$. If uniform coarsening is used, the mapping restriction forces the coarsest multigrid level to have a polynomial order $N_{\text {coarse }} \geq 2$. However, if high-order coarsening is used, the coarsest polynomial order can be as low as $N_{\text {coarse }} \geq 1$ since the two coarsest levels are always $p$-conforming. Having coarser multigrid levels is advantageous since they help to eliminate the low frequency components of the error.

\subsection{The $p$-Adaptation Algorithm: Computing the New Polynomial Orders}

Given a truncation error threshold, $\tau_{\max }$, that needs to be achieved, and a maximum allowed polynomial order, $N_{\max }$, the proposed adaptation process can be summarized in six steps:

1. A high-order discretization, $P=\left(P_{1}, P_{2}, P_{3}\right)$, is converged to a residual $\tau_{\max } / 10$ using the multigrid method described in Section 4.2.2.

2. The anisotropic multigrid procedure of Section 9.2.1 is used to estimate the truncation error contribution in every coordinate direction. For instance, when coarsening in the coordinate direction (1), the contribution is

$$
\mathscr{T}_{1}^{N_{1} N_{2} N_{3}} \approx \mathscr{T}_{P_{1} P_{2} P_{3}}^{N_{1} P_{2} P_{3}}=\mathbf{H}^{N_{1} P_{2} P_{3}}\left(\underline{\mathbf{I}}_{P_{1} P_{2} P_{3}}^{N_{1} P_{2} P_{3}} \tilde{\mathbf{Q}}^{P_{1} P_{2} P_{3}}\right)-\underline{\mathbf{M}}^{N_{1} P_{2} P_{3}} \mathbf{S}^{N_{1} P_{2} P_{3}} .
$$

3. The inner truncation error map (for $N_{i}<P_{i}$ ) is generated using (9.7). 
4. If $\tau_{\max }$ can be achieved using one of these combinations, it is selected and the simulation continues.

5. If $\tau_{\max }$ cannot be achieved in the inner truncation error map, the directional components of the truncation error, $\tau_{i}$, are extrapolated using a linear-log regression Afterwards, the full truncation error map is generated for $P_{i} \leq$ $N_{i} \leq N_{\max , i}$.

6. If $\tau_{\max }$ can be achieved using one of these combinations, the combination is selected. If not, $N_{1}=N_{2}=N_{3}=N_{\max }$ is selected.

In Steps 4 and 6, there can be multiple combinations $\left(N_{1}, N_{2}, N_{3}\right)$ that achieve $\|\tau\|_{\infty}<\tau_{\max }$. In that case, the combination with the lowest number of degrees of freedom is chosen. Notice that the two main differences with the method of Kompenhans et al. [110] are: (i) the way in which the truncation error is estimated for $N_{i}<P_{i}$ (step 3) and later for $N_{i} \geq P_{i}$ (step 5); and (ii) that if the truncation error condition is not met, the element is fully enriched in all coordinate directions, instead of in only one.

\subsection{Multi-Stage Adaptation Process}

In this section, we present a multi-stage adaptation process that uses the $p$-adaptation multigrid method of Section 9.2 and the Full Multigrid (FMG) cycling strategy of Section 4.2.2.1. The multi-stage technique is useful when the maximum allowable polynomial order after adaptation $\left(N_{\max }\right)$ is high and the desired truncation error threshold $\left(\hat{\tau}_{\max }\right)$ is low.

The FMG cycling strategy starts from the coarsest multigrid level and performs V-cycles with an increasing number of multigrid levels to obtain an approximation to the finest solution. The multi-stage adaptation strategy proposed here takes advantage of an FMG-cycle and performs multiple adaptation processes at different polynomial orders $\left(\mathscr{P}_{i}\right)$, as depicted in Figure 9.2. In an adaptation stage at level $\mathscr{P}_{i}$ (red circular markers), a $\tau$-estimation procedure is performed using a $3 \mathrm{~V}$ anisotropic multigrid cycle (Figure 9.1), and subsequently, the polynomial orders are adjusted accordingly, but never to a polynomial order that is higher than $\mathscr{P}_{i+1}$.

The use of low resolution reference solutions, whose resolution is only increased locally as needed during the simulation, differs from previous adaptation strategies based on $\tau$-estimation. Traditionally $[73,110,176]$, the whole domain had to be solved with a considerably high resolution before performing a single-stage adaptation process.

The main advantage of this strategy is that the zones of the domain that only require a low-order approximation are identified early in the simulation and are not enriched. As a result, the overall computational cost to compute the reference solution is reduced. 


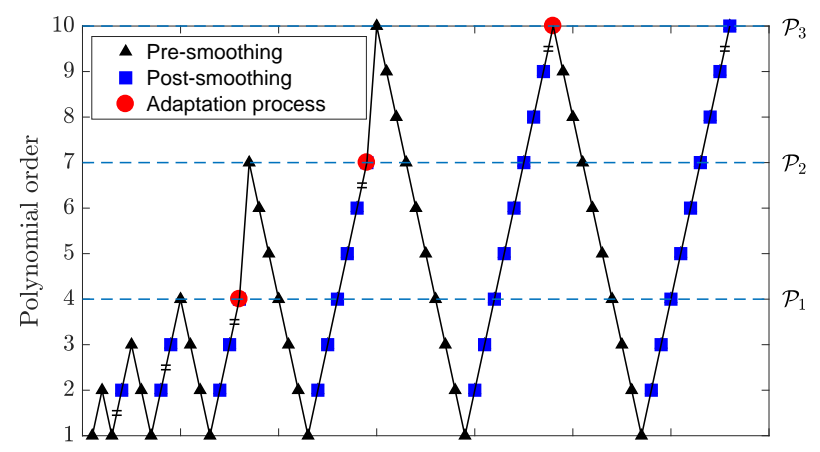

Figure 9.2: Proposed FMG cycle with multiple adaptation stages. Equal signs represent the continuation of the V-cycling process until the desired residual is reached.

After a truncation error estimation at the level $\mathscr{P}_{i}$, the algorithm checks if the maximum required polynomial order is lower or equal to the polynomial order of the next stage, $\mathscr{P}_{i+1}$. If so, the adaptation step that was just performed is marked as the last one and the simulation continues without any further adaptation processes.

\subsection{Numerical Results}

In this section, we test the accuracy and performance of the $p$-adaptation algorithms proposed in this chapter. In Section 9.5.1, the simulation of the flow over a flat plate is used to study the performance of the methods in $2 \mathrm{D}$ problems, and in Section 9.5.2 the simulation of the flow past a sphere is used to analyze the $p$-adaptation methods in $3 \mathrm{D}$ problems.

Since the isolated truncation error is not contaminated by neighbors' errors and it imposes fewer constraints on the error estimation, as seen in Section 7.3.5, we use the isolated truncation error estimator in this section.

All the results presented in this section were obtained using an 8-core $2.6 \mathrm{GHz}$ Intel Xeon E5-2670, 32 GB RAM, and shared memory parallelization (OpenMP + guided schedule with eight threads), as explained in [97]. Note that the parallel implementation has a near-optimal scalability, as can be seen in Appendix C.1.

\subsubsection{D Flow over a Flat Plate}

For this boundary layer test problem, the mesh is constructed using 458 quadrilateral elements, and the simulations use a Reynolds number of $\operatorname{Re}_{\infty}=6000$ (based on the reference length $L=12$ ) and a Mach number of $M_{\infty}=0.2$. Figure 9.3 shows the mesh and the distribution of the momentum in the $\mathrm{x}$ direction, $\rho u$. 


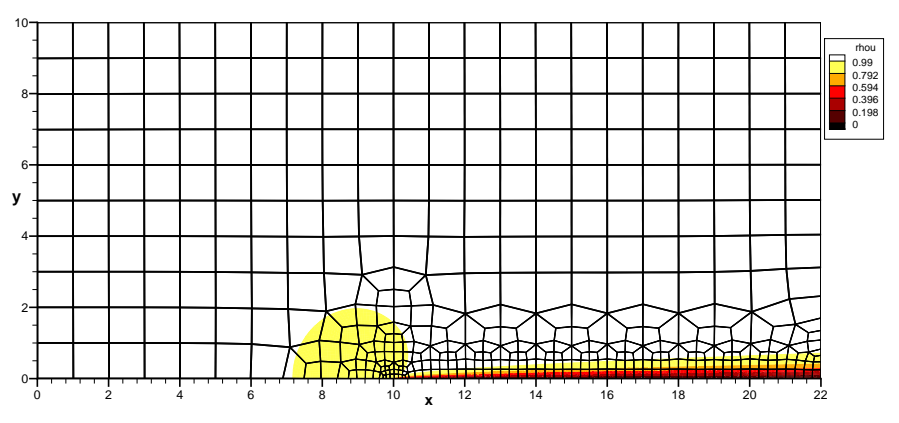

(a) $\mathrm{x}$-Momentum contour.

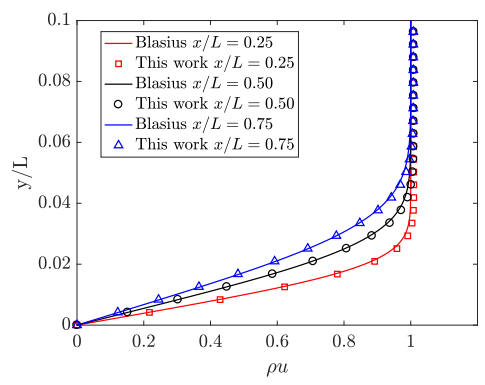

(b) Velocity profiles, $N=10$.

Figure 9.3: Flat plate at $\mathrm{Re}_{\infty}=6000$. $\mathrm{x}$-Momentum contour and velocity profiles at different positions, as compared with the exact solution by Blasius.

A uniform inflow boundary condition was imposed at $x=0$. On the boundary $y=0, x<10$, a free-slip boundary condition was prescribed, whereas for $y=0$, $x \geq 10$, a no-slip adiabatic wall boundary condition emulates the effect of the flat plate. On the remaining boundaries we use a subsonic outflow boundary condition where only the far-field pressure is specified.

\subsubsection{Performance of the Multigrid Method}

In this section, we use a discretization with uniform order, $N_{1}=N_{2}=10$, to assess the performance of a low-storage $3^{\text {rd }}$ order Runge-Kutta scheme (RK3), an isotropic FAS $p$-multigrid procedure, and an anisotropic FAS $p$-multigrid procedure. Both multigrid methods use RK3 as the smoother so that a fair comparison of the performance of solvers can be done.

All the results were obtained using $\beta_{1}^{0}=100$ pre-smoothing sweeps, $\beta_{2}^{0}=100$ post-smoothing sweeps, 400 smoothing sweeps on the coarsest multigrid level and the smoothing tuning explained in Section 4.2.2.2. For all the multigrid simulations of this chapter, the tuning uses $\eta \leq 1.1$, as that value showed to provide enough robustness to the V-cycling strategy for meshes with both uniform polynomial orders and also $p$-anisotropic nonconforming meshes.

In the multigrid simulations, we use an FMG cycling strategy to obtain an appropriate initial condition, where the polynomial order is increased in the FMG cycle using a residual-based strategy: A fixed residual of tol $=10^{-1}$ must be obtained before the polynomial order is raised to the next FMG level. This threshold was selected because it showed to enhance performance in the analyzed problem.

The convergence rate of the multigrid strategies is much higher than that of the completely explicit RK3 time integration, both in number of iterations and in CPUtime. This behavior can be observed in Figure 9.4, where the infinity norm of the residual as a function of the iteration number and the simulation time is plotted 


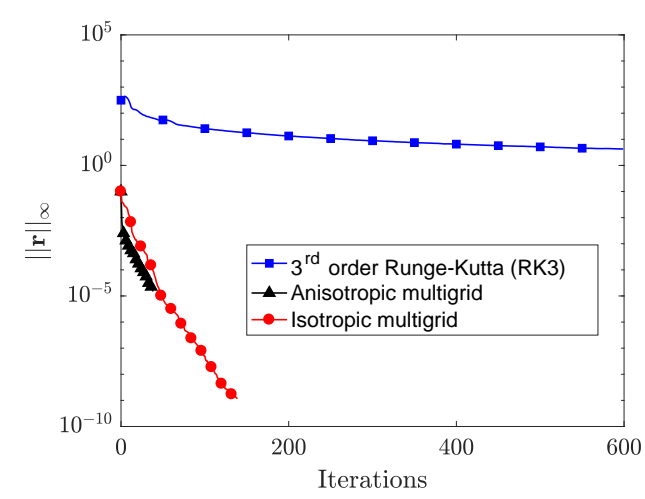

(a) Residual norm vs. iterations.

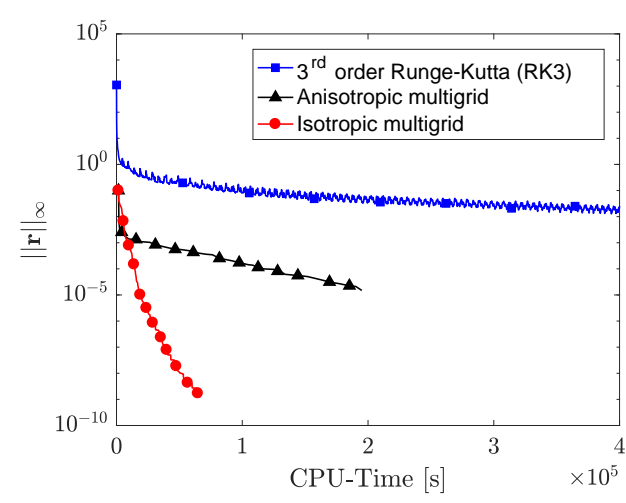

(b) Residual norm vs. CPU-time.

Figure 9.4: Comparison of the performance of an RK3 method and the isotropic / anisotropic FAS $p$-multigrid method (with RK3 as a smoother) for solving the subsonic boundary layer test problem $(N=10)$.

in blue for the RK3 scheme, in red for the isotropic multigrid, and in black for the anisotropic multigrid.

Figure 9.4(a) shows that the isotropic and anisotropic multigrid methods have a similar convergence rate with respect to the number of iterations, the latter being slightly better. Furthermore, it can be seen that RK3 reduces the residual by only a factor of ten over 600 iterations, compared to eight orders of magnitude over 150 iterations for multigrid methods. Nevertheless, this is not a fair comparison since every iteration of a FAS $p$-multigrid method involves several iterations of the RK3.

Figure 9.4(b) presents a fairer comparison between the RK3 and the FAS $p$ multigrid methods. Note that, although the isotropic and anisotropic multigrid methods have a similar convergence rate with respect to the number of iterations, the isotropic FAS multigrid procedure is more efficient than the anisotropic one when the computational time is considered. The reason is that in the isotropic multigrid the lower multigrid levels have fewer degrees of freedom than in the anisotropic multigrid because the coarsening is done in all coordinate directions. For this reason, in next sections the anisotropic FAS will only be used as the anisotropic $\hat{\tau}$-estimator (although during the estimation it is also used as a smoother) and the isotropic FAS will be mainly used as the solver.

\subsubsection{Single-Stage Adaptation}

In this section, we study the computational cost involved in solving the boundary layer test case for different accuracy levels by comparing uniform adaptation with the single-stage adaptation algorithm of Section 9.3. The two solvers used are the RK3 and the FAS solver. The single-stage adaptation process was performed for 
$N_{\max }=10$ and $N_{\max }=5$, with a reference mesh of $P_{1}=P_{2}=4$. Notice that the use of such a coarse discretization as a reference mesh is possible because of the accurate extrapolation of the anisotropic estimation method.

After adapting the mesh, the polynomial order jump across faces is limited to one, i.e.

$$
\left|N_{i}^{+}-N_{i}^{-}\right| \leq 1,
$$

where $N_{i}^{+}$and $N_{i}^{-}$indicate the polynomial order in the coordinate direction $i$ of an element and its neighbor, respectively (the relative rotation between neighboring elements is taken into account). Condition (9.15) provides robustness to the $p$ adapted discretization with respect to the CFL condition, and it is comparable with the two-to-one rule that is usually employed in $h$-adaptation methods $[37,129]$.

Since the anisotropic truncation error estimator (9.7) has been shown to generate more accurate extrapolations of the truncation error map than conventional $\hat{\tau}$-estimators, the single-stage $p$-adaptation method (that is used in all cases) employs a $3 \mathrm{~V}$ anisotropic V-cycle for estimating the isolated truncation error, even when the time-marching solver is RK3.

A higher-order solution of order $N_{1}=N_{2}=15$ was used to estimate the relative error in the drag coefficient,

$$
e_{d r a g}^{N=15}=\frac{\left|C_{d}-C_{d}^{N=15}\right|}{C_{d}^{N=15}},
$$

where $C_{d}^{N=15}=0.211$, a value that is comparable to results in the literatute [133] for a flat plate at $\operatorname{Re}_{\infty}=6000$.

The results obtained with the different methods are illustrated in Figure 9.5. As shown in Figure 9.5(a), the p-adapted meshes require much fewer degrees of freedom for achieving the same error than the uniformly adapted meshes. Note that the minimum relative error, which is achieved for low values of $\hat{\tau}_{\max }$, tends to the relative error that corresponds to a mesh with uniform $N_{\max }$, in the same way as the minimum $\|\hat{\tau}\|_{\infty}$ is a function of $N_{\max }$. This behavior was explained in Section 8.4.3, where we saw that after reaching this plateau, no further improvement in the functional error is expected. The plateau is not necessarily obtained when all elements have $N_{\max }$, as can be seen in Figure 9.6. This is the main advantage of $p$-adaptation methods: It is possible to obtain the maximum accuracy with fewer degrees of freedom if the maximum resolution is only used where needed.

As can be seen in Figure 9.5(b), the $p$-adaptation procedures are especially efficient in computational time when high accuracy is needed. Using a low $N_{\max }$ can lead to faster simulations, but the stagnation point is met sooner. It can also be observed that the most efficient procedure is the one that uses both FAS multigrid and $p$-adaptation. In fact, this combination achieves the maximum accuracy in drag after a two hours of simulation than the classical approach (uniform order + RK3) after several days of computations. Table 9.1 shows the CPU-time comparison of 


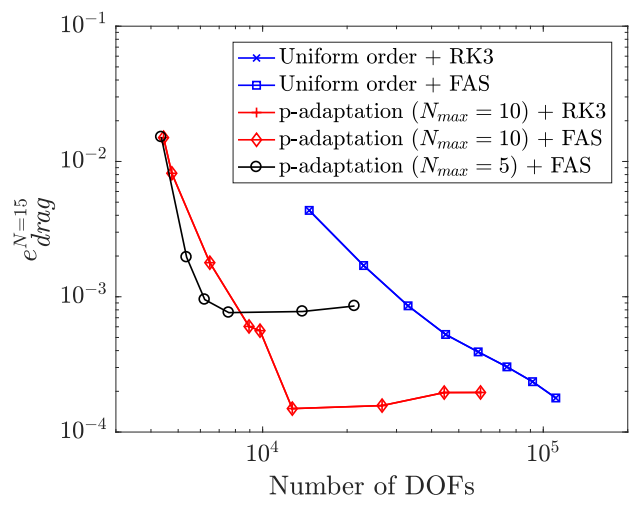

(a) Drag error vs. number of DOFs.

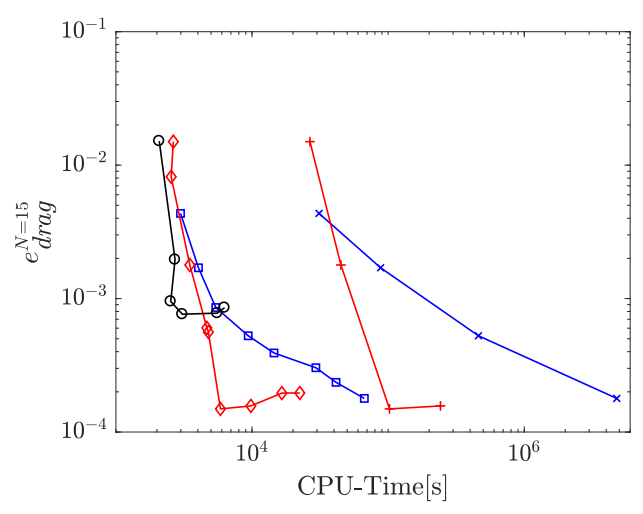

(b) Drag error vs. CPU-Time.

Figure 9.5: Relative error in the drag coefficient calculation for different methods. The reference drag $C_{d}^{P=15}$ was calculated on a uniformly refined mesh with $P=$ 15. The blue lines represent uniform refinement, the red lines represent the $\hat{\tau}$ based $p$-adaptation procedure with $N_{\max }=10$, and the black lines with $N_{\max }=5$. Overlapping curves in (a).

Table 9.1: Computation times and speed-up for four methods to get a relative drag error of at least $1.8 \times 10^{-4}$ after converging to $\|\mathfrak{R}\|_{\infty}<10^{-9}$.

\begin{tabular}{l|rrr} 
Method & CPU-time[s] & Time [\%] & Speed-up \\
\hline \hline RK3 & $4.78 \times 10^{6}$ & $100.00 \%$ & 1.00 \\
RK3 $+p$-adaptation & $1.02 \times 10^{5}$ & $2.14 \%$ & 46.72 \\
FAS & $6.69 \times 10^{4}$ & $1.40 \%$ & 71.51 \\
FAS $+p$-adaptation & $5.86 \times 10^{3}$ & $0.12 \%$ & 815.76
\end{tabular}

different solution procedures for reaching a drag error of at least $1.8 \times 10^{-4}$. The speed-up is as high as 815.76.

Figure 9.6 shows the final polynomial orders computed by the single-stage isolated truncation error estimator for $\hat{\tau}_{\max }=10^{-3}$ (equivalent to a drag error of $e_{d r a g}^{N=15}=1.49 \times 10^{-4}$ ). Most of the polynomial enrichment is performed on the leading edge of the flat plate, around the singularity, and in the regions where the boundary layer grows, as expected. Further polynomial enrichment can be observed in regions where the mesh size changes. 


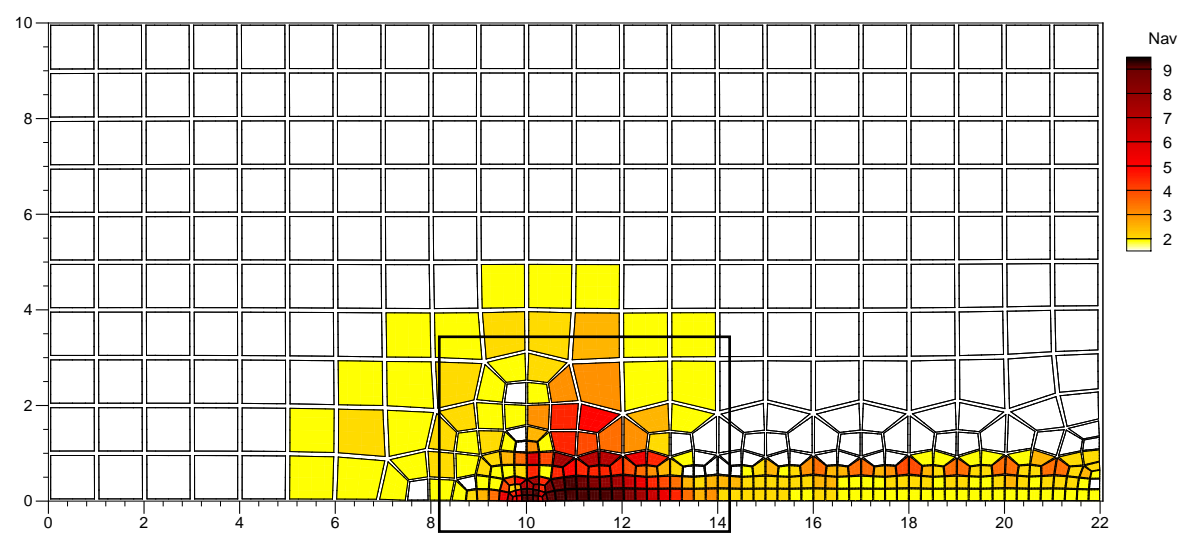

(a) Average polynomial order $\left(N_{a v}\right)$.

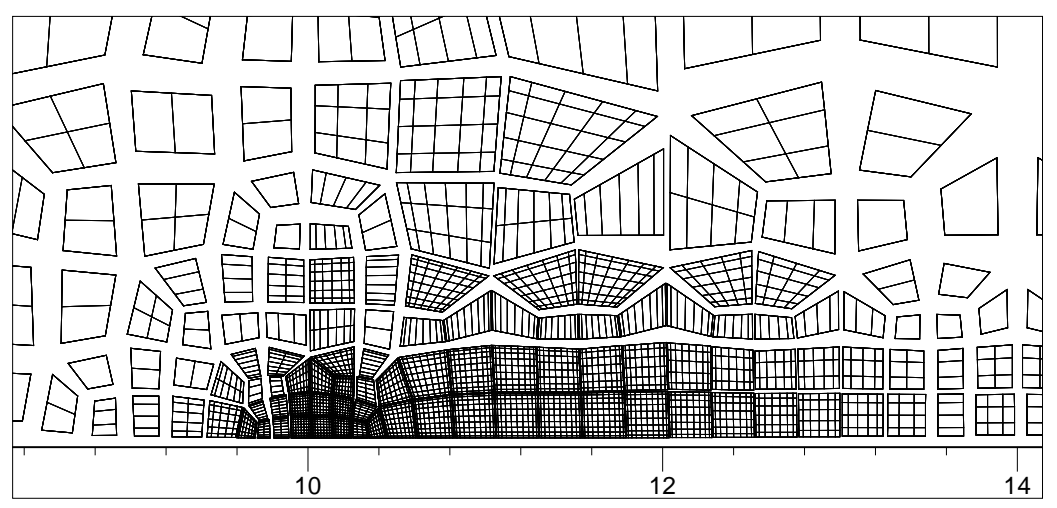

(b) Detail of the Gauss-points.

Figure 9.6: Contour indicating the final average polynomial orders after the adaptation procedure (a) and a detail of the Gauss-Points that shows the anisotropic nature of the $p$-adaptation method (b) for a threshold of $\hat{\tau}_{\max }=10^{-3}$, which produces a relative drag error of $e_{d r a g}^{N=15}=1.49 \times 10^{-4}$. White boxes represent $N_{1}=N_{2}=1$. $N_{a v}=\left(N_{1}+N_{2}\right) / 2$. 


\subsubsection{Multi-Stage Adaptation}

We now apply the multi-stage $p$-adaptation procedure that uses a full multigrid scheme (which was presented in Section 9.4) to the boundary layer test problem and see when it provides advantages.

Choosing a higher $N_{\max }$ increases the accuracy (as was seen in last section), but it also requires us to make additional considerations. A higher $N_{\max }$ means that a larger truncation error map must be computed. This means that more storage is needed, that more operations are needed to compute the values of the map, and that we have either to rely on extrapolations or to increase the order of the reference solution, $P$, which requires additional computational resources.

The problem with extrapolations is that their accuracy is highly dependent on the reference polynomial order, $P$. As we increase the area of the map where we have to extrapolate the values, the uncertainty of the estimations also increases. Figure 9.7 shows the number of degrees of freedom in the mesh after a single-stage adaptation procedure $\left(9 \times 10^{-4} \leq \hat{\tau}_{\max }<10^{-1}\right.$ and $\left.N_{\max }=20\right)$ for reference meshes of different order. As can be seen, the number of DOFs increases at a higher rate when the specified truncation error is reduced below a specific value. This value is higher for low-order reference meshes where, because of errors in the extrapolation procedure, the truncation error is over-predicted and some elements are over-enriched to $N_{\max }$.

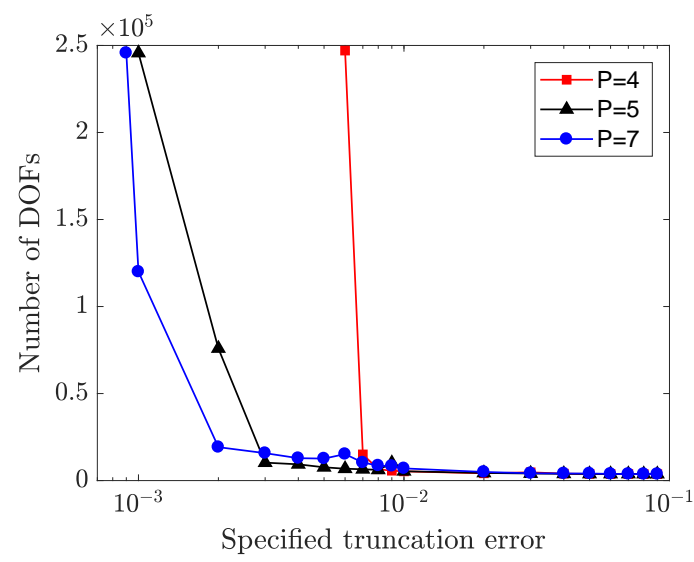

Figure 9.7: Number of degrees of freedom after adapting the mesh with different thresholds $\left(\hat{\tau}_{\max }\right)$ and different reference meshes $(P)$ for $N_{\max }=20$.

For high values of $N_{\max }$, a multi-stage $p$-adaptation procedure becomes very useful. As was explained in Section 9.4, instead of starting with a high-order reference mesh (which can be very expensive), a coarse reference mesh of order $P=\mathscr{P}_{1}$ is chosen to estimate the truncation error. With the estimate, the regions where a low-order approximation is enough are identified. Afterwards, the $p$-adaptation algorithm sets the polynomial orders of the mesh according to the $\hat{\tau}$-estimation 
and limits the over-enrichment in more complex flow regions to $\mathscr{P}_{2}$. In the second adaptation process at $P=\mathscr{P}_{2}$, and in subsequent adaptation stages, the polynomial orders of the mesh are corrected with a more accurate error estimate at hand.

To illustrate how the multi-stage adaptation method can reduce the computational cost of highly accurate simulations, we present a comparison of the convergence of the single-stage and the multi-stage $p$-adaptation procedures for $\hat{\tau}_{\max }=$ $4 \times 10^{-3}$ and $N_{\max }=20$ in Figure 9.8(a), and for $N_{\max }=30$ in Figure 9.8(b). The reference meshes of the multi-stage algorithm were selected at $\mathscr{P}_{1}=4, \mathscr{P}_{2}=8$ and $\mathscr{P}_{3}=16$. The measured speed-up is 1.69 for $N_{\max }=20$, and 1.72 for $N_{\max }=30$ with respect to the single-stage adaptation.

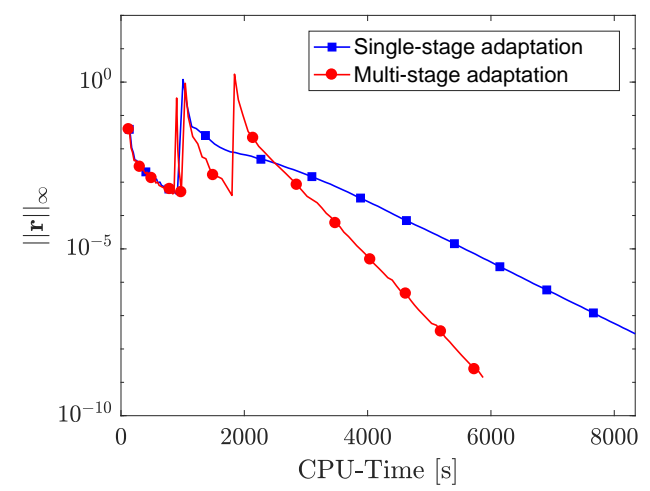

(a) $N_{\max }=20$.

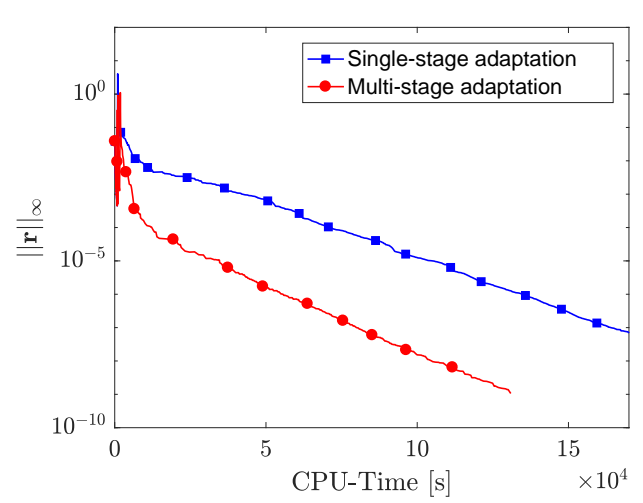

(b) $N_{\max }=30$.

Figure 9.8: Comparison of a single-stage and a multi-stage adaptation process for solving the boundary layer test case with a truncation error threshold of $\hat{\tau}_{\max }=$ $4 \times 10^{-3}: N_{\max }=20(\mathrm{a})$, and $N_{\max }=30$ (b).

\subsubsection{D Flow Around a Sphere}

For this test, the mesh is constructed with 1904 hexahedral elements, and the simulations are computed with a Reynolds number of $\operatorname{Re}_{\infty}=200$ and a Mach number of $\mathrm{M}_{\infty}=0.2$. The curvilinear hexahedral mesh has a mapping order $M=3$ and was created using the HOPR package [96]. Figure 9.9 shows the mesh and the distribution of the conserved variable $\rho u$ around the sphere.

To assess error of the adapted meshes, we use a relative drag error that is computed against a high-order solution of order $N=12$ on the same mesh,

$$
e_{d r a g}^{N=12}=\frac{\left|C_{d}-C_{d}^{N=12}\right|}{C_{d}^{N=12}} .
$$

Table 9.2 shows a comparison between the reference drag coefficient obtained in this work and in other studies. 
Table 9.2: Drag coefficient for sphere at $\operatorname{Re}_{\infty}=200$.

\begin{tabular}{lr} 
Author & Value \\
\hline \hline Campregher et al. [41] & 0.815 \\
Fornberg [70] & 0.7683 \\
Fadlun et al. [59] & 0.7567 \\
This work $(N=12)$ & 0.7771
\end{tabular}

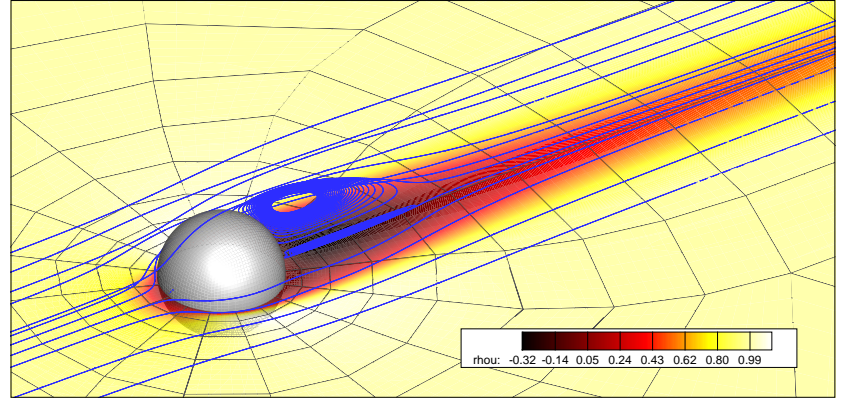

(a) x-Momentum contour.

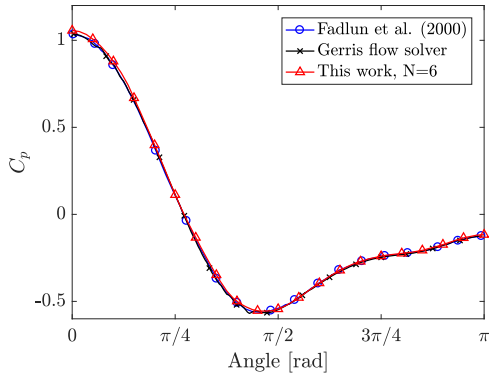

(b) Pressure coefficient.

Figure 9.9: Sphere at $\operatorname{Re}_{\infty}=$ 200. x-Momentum contour and pressure coefficient $\left(C_{p}\right)$ as compared with data by Fadlun et al. [59] and the Gerris flow solver [162].

\subsubsection{3D Considerations}

In the general 3D $p$-nonconforming DGSEM, the mapping order in every reference coordinate direction, $M_{i}$, is limited by the solution order as

$$
M_{i} \leq N_{i} / 2
$$

for the discretization to be free-stream preserving, as indicated in Section 2.2. Furthermore, since the $p$-adapted meshes are usually $p$-nonconforming, the minimum polynomial order after $p$-adaptation is set to $N_{\text {min }}=2$. Additionally, as noted in Section 9.2.2, we employ a $p$-multigrid method with high-order coarsening, as it allows us to use $N_{\text {coarse }}=1$ without violating condition (9.18).

We want to represent the curved boundary on the sphere as exactly as possible, i.e. to maintain the mapping order, $M$, as high as it can be. To avoid the strong constraints on the geometry representation that condition (9.18) imposes, we use a conforming algorithm, which changes the polynomial orders of all elements on that surface after the $p$-adaptation, so that there is no polynomial order jump across their faces. When the discretization is completely conforming on the curved surface, a mapping of order $M_{i} \leq N_{i}$ can be used there without having free-stream preservation issues. 
Finally, let us remark that in 3D, the condition of (9.15), which enforces a polynomial jump across faces of 1 , can cause a steep increase in the number of degrees of freedom because the polynomial enriching is transmitted in three coordinate directions. Therefore, for this test case the polynomial order jump across faces after $p$-adaptation is softened to

$$
N_{i}^{+} \geq\left\lfloor\frac{2}{3} N_{i}^{-}\right\rfloor
$$

where $\lfloor\cdot\rfloor$ is the integer part floor function.

Condition (9.19) provided enough robustness to the $p$-adapted discretizations with respect to the CFL number and lowered the number of degrees of freedom of the adapted meshes. The conforming algorithm that is used on the sphere boundary and the algorithm that controls the polynomial order jump everywhere must be executed iteratively, until no further changes are needed, to ensure that the final mesh has all the desired properties.

\subsubsection{Single-Stage Adaptation}

The single-stage $p$-adaptation algorithm that uses $p$-anisotropic truncation error estimates reduces the computational resources (storage and computing time) that are needed to solve the 3D flow around a sphere. The performance of the singlestage strategy is assessed for $N_{\max }=7$, where a reference mesh of order $P_{1}=P_{2}=$ $P_{3}=5$ is used. The truncation error threshold was tested in the range $10^{-1} \leq \hat{\tau}_{\max } \leq$ $10^{-4}$.

Following parameters were used for the simulations. The isotropic and conforming reference mesh is converged to a residual of $\|\tilde{\mathfrak{R}}\|_{\infty} \leq \hat{\tau}_{\text {max }} / 10$ using a $p$-multigrid algorithm with $\beta_{1}^{0}=\beta_{2}^{0}=100$ pre- and post-smoothing sweeps, and 400 smoothing sweeps on the coarsest multigrid level. After the $p$-adaptation, the pre- and post-smoothing sweeps are $\beta_{1}^{0}=\beta_{2}^{0}=50$, and the number of smoothing sweeps on the coarsest multigrid level is 200 (this combination exhibited the best performance). The smoothing tuning detailed in Section 4.2.2.2 is used and an FMG cycling strategy is employed to obtain an appropriate initial condition with a residual of $\|\tilde{\mathfrak{R}}\|_{\infty} \leq 1.0$.

Figure 9.10 shows a comparison between the lift and drag errors obtained using the $\hat{\tau}$-based adaptation procedure and the ones using uniform $p$-refinement. As could be expected, the maximum error in lift and drag corresponds to the one obtained with a uniform mesh of $N_{1}=N_{2}=N_{3}=N_{\text {min }}=2$. Similarly, the minimum error in lift coefficient tends to $\left|C_{l}^{N=7}\right|$ and the minimum error in drag tends to $e_{\text {drag }}(N=7)$. Namely, as the error threshold, $\hat{\tau}_{\max }$, is decreased, the polynomial orders of the mesh tend to the maximum specified polynomial order, $N_{\max }=7$. Depending on factors like small asymmetries of the discretization, discrepancies in the error estimation, among others, the absolute lift might fall below $\left|C_{l}^{N_{\max }}\right|$ for some intermediate thresholds. However, the expected value for $\|\hat{\tau}\|_{\infty} \rightarrow 0$ is $C_{l}^{N_{\max }}$. 
The number of degrees of freedom is reduced for any accuracy when using the $\hat{\tau}$-based $p$-adaptation, compared to uniform refinement, as can be observed in Figures 9.10(a) and 9.10(b). Namely, the minimum drag error, $e_{\text {drag }} \leq 5.3 \times 10^{-4}$, is obtained on the $p$-adapted meshes with only $6 \times 10^{4}$ degrees of freedom, whereas the uniformly refined meshes need $10^{6}$ degrees of freedom to reach that accuracy. Similarly, the minimum lift, $\left|C_{l}\right| \leq 3.3 \times 10^{-4}$, is obtained with $8 \times 10^{4}$ degrees of freedom on the $p$-adapted meshes, whereas the uniformly refined meshes need $10^{6}$ degrees of freedom to reach that accuracy.

The computation times for the $\hat{\tau}$-based $p$-adaptive simulations are shorter than those for the uniformly refined simulations when high accuracy is sought (in this case $\left|C_{l}\right| \leq 5 \times 10^{-3}$ and $e_{d r a g} \leq 10^{-2}$ ), as seen in Figures 9.10(c) and 9.10(d). It is interesting to notice that for high truncation error thresholds, the $\hat{\tau}$-based adaptation does not provide an advantage in CPU-time. This occurs because the cost to obtain a semi-converged solution on the reference mesh of $P=5$ is not counterbalanced with a reduction of the computation time, e.g. when the final polynomial order post-adaptation is $N<5$.

Using the data provided by Figures $9.10(\mathrm{c})$ and $9.10(\mathrm{~d})$, it is possible to compute the speed-up as a function of the drag or lift errors. Tables 9.3 and 9.4 show the computation times and speed-ups achieved for the lowest error obtained in lift and drag $\left(e_{d r a g}^{N=12}\right.$ and $\left.\left|C_{l}\right|\right)$. The maximum speed-up is 151.94 for this challenging 3D flow.

Table 9.3: Computation times and speed-up to get a drag error of $e_{\text {drag }} \leq 5.31 \times 10^{-4}$ (the lowest achieved). All simulations are converged to $\|\mathfrak{R}\|_{\infty}<10^{-9}$.

\begin{tabular}{l|rrr|} 
Method & CPU-time[s] & Time [\%] & Speed-up \\
\hline \hline RK3 & $7.46 \times 10^{6}$ & $100.00 \%$ & 1.00 \\
FAS & $2.72 \times 10^{5}$ & $3.65 \%$ & 27.41 \\
FAS $+p$-adaptation & $4.91 \times 10^{4}$ & $0.68 \%$ & 151.94
\end{tabular}

Table 9.4: Computation times and speed-up to get a lift error of $\left|C_{l}\right| \leq 3.34 \times 10^{-4}$ (the lowest achieved). All simulations are converged to $\|\mathfrak{R}\|_{\infty}<10^{-9}$.

\begin{tabular}{l|rrr|} 
Method & CPU-time[s] & Time [\%] & Speed-up \\
\hline \hline RK3 & $7.46 \times 10^{6}$ & $100.00 \%$ & 1.00 \\
FAS & $2.72 \times 10^{5}$ & $3.65 \%$ & 27.41 \\
FAS $+p$-adaptation & $5.80 \times 10^{4}$ & $1.06 \%$ & 128.55
\end{tabular}

Figure 9.11 shows the polynomial order distribution after $p$-adaptation for $\hat{\tau}_{\max }=$ $4 \times 10^{-4}$, which corresponds to a drag error of $e_{\text {drag }}=8.08 \times 10^{-4}$ and an absolute 


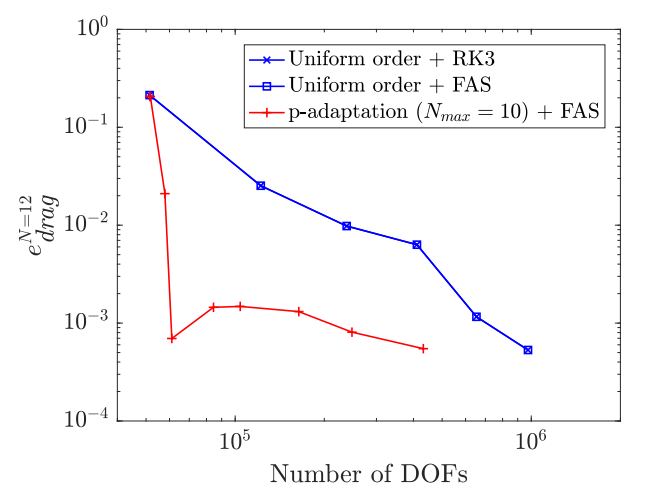

(a) Drag error vs. number of DOFs.

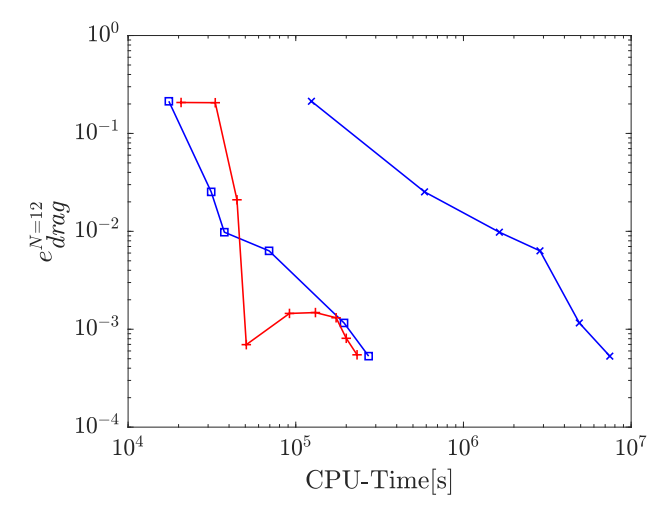

(c) Drag error vs. CPU-Time.

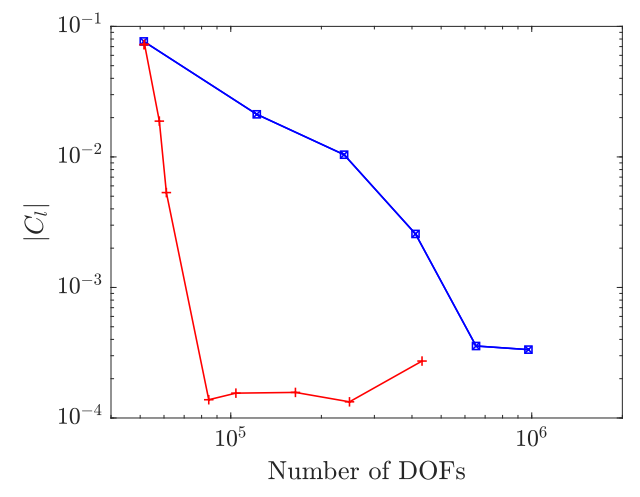

(b) Absolute lift vs. number of DOFs.

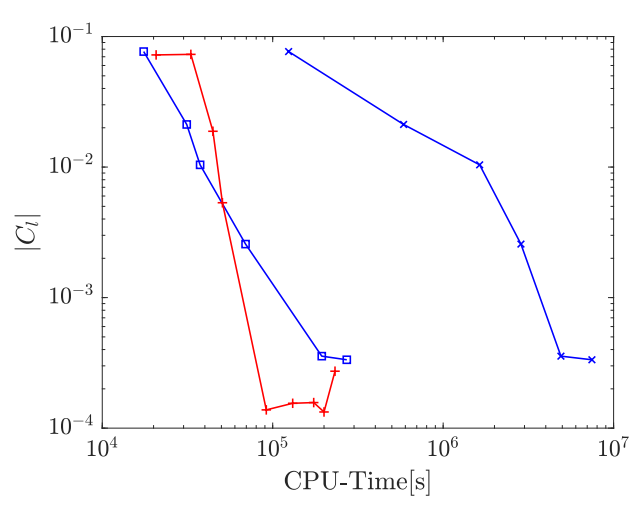

(d) Absolute lift vs. CPU-Time.

Figure 9.10: Relative error in the drag and lift coefficients for the sphere flow problem. The blue lines represent uniform refinement, and the red lines represent the $\hat{\tau}$-based $p$-adaptation procedure with $N_{\max }=7$. 
lift of $\left|C_{l}\right|=1.33 \times 10^{-4}$. It can be seen that intensive polynomial enrichment is performed in the recirculation bubble, the wake, and in the boundary layer, as expected. Further polynomial enrichment can be observed in regions where the mesh size changes drastically. In particular, we observe that the polynomial enrichment is higher in the region that connects the wake with the recirculation bubble than in the boundary layer. Indeed, the original $h$-mesh showed large elements downstream of the sphere, which required local refinement. This over-enrichment of the recirculation bubble increases the number of degrees of freedom and may explain why the the speed-up is not as high as in the flat plate case: The zone that is expected to contribute the most to the lift and drag is the boundary layer, which is enriched to a lesser extent.

The slight asymmetries are due to small discrepancies in the error estimation and the fact that the polynomial order is a discrete function: A slight difference in the error estimate can cause important differences in polynomial orders. In addition, the relative rotation between neighboring elements can also affect the truncation error estimation process in the anisotropic coarsening on a small scale. Although this effect is more important for the non-isolated truncation error estimation, it is also weakly present for the isolated truncation error.

The rate of convergence in CPU-time deteriorates after the $p$-adaptation, as the $p$-anisotropic nonconforming discretizations are more difficult to solve. Further investigation on multigrid, or other solution methods, could help solve this decrease in the rate of convergence and, hence, improve the speed-ups reported here.

\subsubsection{Multi-Stage Adaptation}

The multi-stage adaptation procedure introduced in Section 9.4 becomes useful when the maximum allowable polynomial order after adaptation $\left(N_{\max }\right)$ is increased and the specified isolated truncation threshold $\left(\hat{\tau}_{\max }\right)$ is low. In this section, we use the multi-stage $p$-adaptation procedure on the sphere and set the maximum polynomial order after adaptation to $N_{\max }=11$, the truncation error threshold to $\hat{\tau}_{\text {max }}=10^{-4}$, and the adaptation stages to $\mathscr{P}_{1}=4$ and $\mathscr{P}_{2}=8$. Figure 9.12 shows a comparison of performance (in CPU-Time) between the multi-stage $p$-adaptation procedure and two single-stage procedures with $P=4$ and $P=5$. The maximum polynomial order after adaptation in the single stage cases is also $N_{\max }=11$.

Figure 9.12 shows that the convergence rate (with respect to CPU-time) of the multi-stage $p$-adapted mesh is higher than for single-stage $p$-adapted meshes since the former has fewer degrees of freedom. For this reason, the multi-stage $p$-adapted mesh is able to achieve a speed-up of 2.69 compared to the single-stage method to converge to a near-round-off residual $\|\tilde{\mathfrak{R}}\|_{\infty}=10^{-9}$. Table 9.5 shows a summary of results for the simulations of this section.

The number of degrees of freedom for the single-stage $P=4$ is the highest, since in that case many elements are enriched to the maximum $N_{1}=N_{2}=N_{3}=11$ due to overshoots in the error estimate, as explained in Section 9.5.1.3). In the 


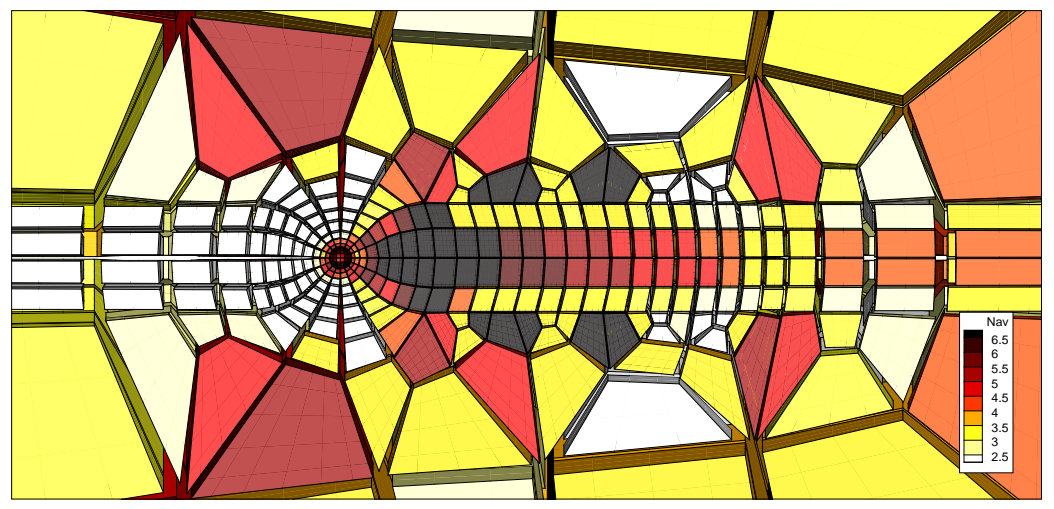

(a) Average polynomial order $\left(N_{a v}\right)$.

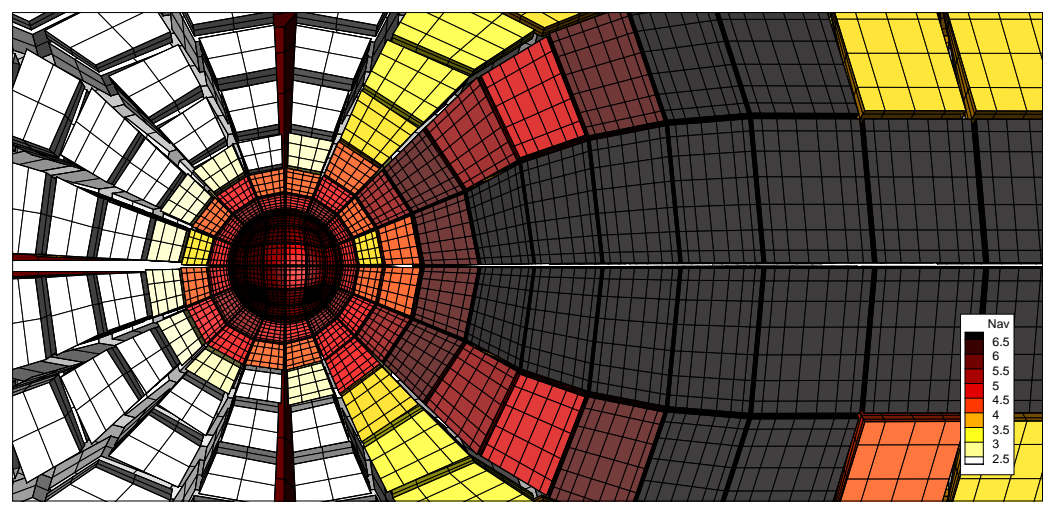

(b) Detail of the Gauss-points.

Figure 9.11: Contours indicating the final polynomial orders after $p$-adaptation for the sphere test case: Average polynomial orders $\left(N_{a v}\right)$ (a) and a detail of the Gauss-Points that shows the anisotropic nature of the $p$-adaptation method (b) for a threshold of $\hat{\tau}_{\max }=4 \times 10^{-4}$. White boxes represent $N_{1}=N_{2}=N_{3}=2$.. $N_{a v}=\left(N_{1}+N_{2}+N_{3}\right) / 3$.

Table 9.5: Summary of performance for single- and multi-stage simulations with $\hat{\tau}_{\max }=10^{-4}$

\begin{tabular}{l|ccccc} 
Strategy & DOFs & CPU-Time(s) & Speed-up & $e_{\text {drag }}$ & $\left|C_{l}\right|$ \\
\hline \hline S. Stage $P=4$ & $1.07 \times 10^{6}$ & $4.53 \times 10^{5}$ & 1.00 & $2.27 \times 10^{-5}$ & $1.39 \times 10^{-5}$ \\
\hline S. Stage $P=5$ & $7.90 \times 10^{5}$ & $2.68 \times 10^{5}$ & 1.69 & $3.57 \times 10^{-5}$ & $1.90 \times 10^{-4}$ \\
\hline Multi-Stage & $1: 6.20 \times 10^{5}$ & $1.75 \times 10^{5}$ & 2.59 & $4.50 \times 10^{-5}$ & $2.12 \times 10^{-5}$ \\
$\mathscr{P}=[4,8]$ & $2: 3.85 \times 10^{5}$ & & & &
\end{tabular}




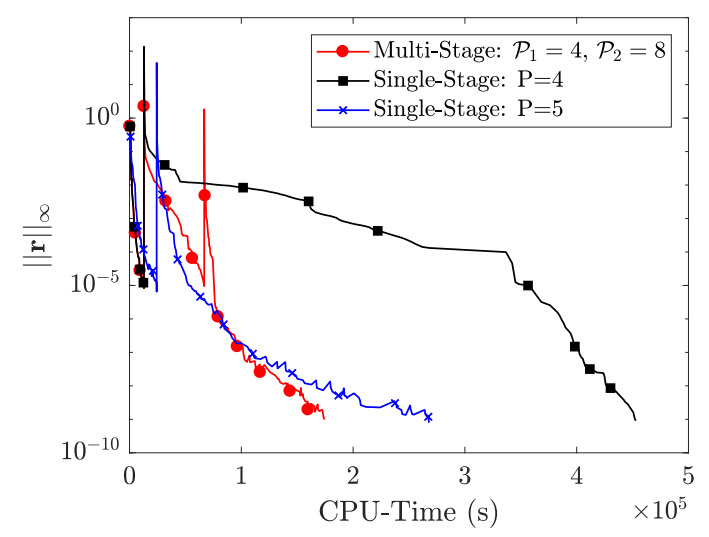

Figure 9.12: Comparison of single-stage $(P=4$ and $P=5)$ and multi-stage adaptation $\left(\mathscr{P}_{1}=4, \mathscr{P}_{2}=8\right)$ processes for the sphere. $N_{\max }=11, \hat{\tau}_{\max }=10^{-4}$.

single-stage $P=5$ this behavior is also observed, but to a lesser extent.

The number of degrees of freedom in the first stage of the multi-stage method is limited by the condition $N_{\max , 1}=8$, and the distribution of polynomial orders is then corrected in the second stage, where the number of degrees of freedom decreases, even though the maximum polynomial order is $N_{\max , 2}=11$.

It is remarkable that the multi-stage adapted mesh can achieve comparable drag and lift errors with about one third of the degrees of freedom and a speed-up of 2.59 with respect to the single-stage $P=4$.

\subsection{Final Remarks}

In this chapter, a solver using truncation error estimators, anisotropic $p$-adaptation and multigrid was developed. The most important results of this work are:

1. A novel anisotropic $p$-adaptation multigrid algorithm is presented, which uses the multigrid method both as a solver and as a truncation error estimator.

2. The coupling of single-stage $p$-adaptation strategies and multigrid methods resulted in a speed-up of 816 for a 2D boundary layer case and of 152 for the 3D sphere case.

3. The technique to evaluate the truncation error that is presented in Chapter 8 can be performed directly inside an anisotropic multigrid procedure, needing only a few additional operations.

4. Isotropic multigrid methods show better performance than anisotropic multigrid methods. The reason is that the successive coarse grids are cheaper to compute when the polynomial order is reduced in all coordinate directions. 
5. A multi-stage $p$-adaptation technique that couples $\tau$-estimations and multigrid was developed. Experiments show that multi-stage is advantageous for highly accurate simulations compared with single-stage adaptation procedures.

The multi-stage procedure showed to be a promising alternative for 3D simulations, since coarser reference meshes can be used: the elements that do not need to be enriched are identified early and their polynomials are frozen to a low value. The achieved speed-ups with this methods were as high as 2.59 with respect to the single-stage adaptation.

The isotropic versions of the $p$-multigrid strategy and the $\tau$-based $p$-adaptation procedure can be extended to any nodal or modal DG scheme on triangular or tetrahedral elements, where the isotropic multigrid can be readily used as an isotropic $\tau$-estimator. On the other hand, the anisotropic versions of these methods require tensor-product basis functions.

To the author's knowledge, there are two kinds of mapping strategies that retain tensor-product bases in triangular elements. The most known one employs collapsed-edge quadrilaterals, as in $[172,187]$. Another option divides one physical triangular edge into two edges [126, Figure 3]. The anisotropic truncation error estimator, $p$-multigrid procedures and adaptation strategies that are explored in this chapter can be implemented in triangles/tetras/pyramids using any of these mappings.

All the methods of this chapter rely on the anisotropic $\tau$-estimation, which can be done in any DG method that uses tensor-product bases. Therefore, although the $p$-adaptation methods are tested for the DGSEM, they are applicable to any high-order DG method that uses tensor-product basis functions. 

Fow 10

\section{Unsteady p-Adaptation Using Truncation Error Estimates}

\section{Contents}

10.1 Introduction . . . . . . . . . . . . . . . . . . . . . . . 167

10.2 Formulation of the Truncation Error . . . . . . . . . . . . . 169

10.2.1 Traditional Formulation . . . . . . . . . . . . . . . 169

10.2.2 New Formulation . . . . . . . . . . . . . . . . 170

10.3 Truncation Error Estimation in Unsteady Problems . . . . . 172

$10.4 p$-Adaptation Strategies $\ldots \ldots \ldots \ldots$. . . . . . . 173

10.4.1 Dynamic p-Adaptation . . . . . . . . . . . . . . . . . 174

10.4.2 Static $p$-Adaptation . . . . . . . . . . . . . . . . . 175

10.5 Numerical Results . . . . . . . . . . . . . . . 178

10.5.1 Advection of a Vortex in a Uniform Flow . . . . . . . . . 178

10.5.2 Subsonic Flow Past a Cylinder . . . . . . . . . . . . 181

10.6 Final Remarks $\ldots \ldots \ldots \ldots \ldots \ldots$

\subsection{Introduction}

In this chapter, the $\tau$-estimation method is extended to unsteady problems and it is successfully utilized to adapt the polynomial orders of a DGSEM discretization locally and anisotropically, so that the relevant unsteady flow features are captured accurately. This is a novel contribution, as a thorough search of the relevant literature found no applications of the $\tau$-estimation method to unsteady adaptive DG simulations. 
To achieve an efficient unsteady $\tau$-based $p$-adaptation scheme, we revise the definition of the truncation error and propose a new formulation. Furthermore, we present and analyze two different strategies to estimate the truncation error in unsteady problems. Moreover, a static and a dynamic $p$-adaptation methods are presented and tested in unsteady simulations of the compressible Euler and Navier-Stokes equations.

In Section 10.2, we show that the truncation error can be formulated in several forms, depending on the choice of the continuous and discrete partial differential operators. Herein, a thorough analysis of the formulation that is traditionally used in the DG community $[110,111,176,178,180]$ is presented, a new formulation for the truncation error is proposed, and a comparative analysis of both is detailed. The new formulation performs better than the traditional one for the problems presented in this chapter, as will be shown in the Numerical Results section.

Section 10.3 provides two strategies to estimate the truncation error in unsteady problems, both of which are derived from the $\tau$-estimation method. The first strategy is directly derived from the variational DG formulation, is directly obtainable from the fine-grid DG discretization, and does not require changes to the temporal discretization. The second strategy uses a dual time-stepping pseudo-time discretization. Although the second strategy requires the adoption of a specific time-marching scheme, it allows unsteady $p$-adaptation problems to be treated as if they were steady problems. As a result, all the algorithms that have been developed for steady state, e.g. in Chapters 9 and 8, can be used.

In Section 10.4, two $p$-adaptation algorithms for unsteady problems are proposed, which use an unsteady $\tau$-estimation method. First, a dynamic $p$-adaptation strategy is described, which performs several stages of estimation and $p$-adaptation throughout a simulation. This is an effective approach, but it has technical challenges, such as the development of efficient load balancing techniques and the implementation of low-cost truncation error estimators. Second, a static $p$-adaptation strategy is introduced, which performs several truncation error estimation stages, but only one $p$-adaptation stage. This approach is useful in statistically steady flows and provides several implementation advantages with respect to the dynamic $p$ adaptation, but with some limitations. A detailed discussion on the advantages and limitations of both methods is presented.

The methods are applied to unsteady problems modeled by the compressible Euler and Navier-Stokes equations in Section 10.5, and a detailed analysis of their performance is presented. First, we show the performance of both the static and the dynamic $\tau$-based $p$-adaptation methods when solving the advection of an inviscid vortex through a domain. In that problem, the dynamic $p$-adaptation method is more efficient than the static since the flow features move through a large part of the physical domain. Then, we compare both $\tau$-based $p$-adaptation methods when simulating the vortex shedding past a cylinder. In this problem, the static $p$-adaptation method works better since it is a statistically steady flow where the difficult-to-solve flow features are concentrated in a small part of the physical do- 
main.

Finally, the most important findings of this chapter are summarized in Section 10.6 .

\subsection{Formulation of the Truncation Error}

In this section, we show that the mathematical formulation of the truncation error can expressed in several ways while maintaining consistency with the original definition (see Def. 7.4 and 7.5),

$$
\tau^{N}=\mathfrak{r}^{N}\left(\mathbf{I}^{N}(\mathbf{q})\right)-\mathfrak{r}(\mathbf{q}),
$$

where $\mathfrak{r}^{N}$ is a discrete partial differential operator and $\mathfrak{r}$ is the exact partial differential operator, and $\mathbf{q}$ is the exact solution.

Throughout this thesis, and in previous works [110, 111, 176, 178, 180], a specific definition of the differential operators has been used, which will be referred to as the traditional formulation. In section 10.2.1, we analyze the traditional formulation and extend it to unsteady problems. In Section 10.2.2, we introduce a new formulation that is also consistent with (10.1).

\subsubsection{Traditional Formulation}

Traditionally, the discrete partial differential operator has been defined point-wise using the variational formulation,

$$
\mathfrak{r}_{j}^{N}\left(\mathbf{I}^{N}(\mathbf{q})\right)=\int_{\partial \Omega^{e}}^{N} \hat{\mathbf{f}}\left(\mathbf{I}^{N}(\mathbf{q}), \mathbf{I}^{N}(\mathbf{q}), \vec{n}\right) \phi_{j} \mathrm{~d} S \cdot-\int_{\Omega^{e}}^{N} \overleftrightarrow{\mathbf{f}}\left(\mathbf{I}^{N}(\mathbf{q})\right) \cdot \vec{\nabla} \phi_{j} \mathrm{~d} \Omega-\int_{\Omega^{e}}^{N} \mathbf{s}^{N} \phi_{j} \mathrm{~d} \Omega
$$

which is a natural choice when using nodal variational formulations (DG or others) When we are analyzing the non-isolated truncation error, (10.2) allows us to write the discrete PDE in sampled form,

$$
\underline{\mathbf{M}} \frac{\mathrm{d} \mathbf{Q}^{N}}{\mathrm{~d} t}+\mathfrak{R}^{N}\left(\mathbf{Q}^{N}\right)=\mathbf{0} .
$$

To maintain consistency, the continuous partial differential operator must be defined in integral form as well. For steady problems, it was advantageous to define it using the exact integrals as

$$
\left.\mathfrak{r}_{j}(\mathbf{q})\right|_{\text {steady }}=\int_{\partial \Omega^{e}} \stackrel{\mathbf{f}}{\vec{n}} \phi_{j} \mathrm{~d} S .-\int_{\Omega^{e}} \overleftrightarrow{\mathbf{f}}(\mathbf{q}) \cdot \vec{\nabla} \phi_{j} \mathrm{~d} \Omega-\int_{\Omega^{e}} \mathbf{s} \phi_{j} \mathrm{~d} \Omega,
$$

since that term, the exact residual, takes the value of zero in steady state and, therefore, can be neglected. However, since in unsteady problems that term does 
not vanish, we want an equation of the form of (10.3) for the continuous solution,

$$
\underline{\mathbf{M}} \frac{\mathrm{d} \mathbf{Q}}{\mathrm{d} t}+\mathfrak{R}(\mathbf{Q})=\mathbf{0},
$$

where $\mathbf{Q}=\boldsymbol{I}^{N} \mathbf{q}$ is the sampled exact solution, as defined in Section 7.3.4.

An equation of the form (10.5) can be derived if we use a quadrature rule instead of the exact integrals,

$$
\left.\mathfrak{r}_{j}(\mathbf{q})\right|_{\text {unsteady }}=\int_{\partial \Omega^{e}}^{N} \overleftrightarrow{\mathbf{f}} \cdot \vec{n} \phi_{j} \mathrm{~d} S .-\int_{\Omega^{e}}^{N} \overleftrightarrow{\mathbf{f}}(\mathbf{q}) \cdot \vec{\nabla} \phi_{j} \mathrm{~d} \Omega-\int_{\Omega^{e}}^{N} \mathbf{s} \phi_{j} \mathrm{~d} \Omega .
$$

The similarities between (10.3) and (10.5), together with the definition of the truncation error, allow one to write the Discretization Error Transfer Equation (DETE) in sampled form,

$$
\underline{\mathbf{M}} \frac{\mathrm{d} \mathscr{E}}{\mathrm{d} t}+\left[\mathfrak{R}^{N}\left(\mathbf{I}^{N} \mathbf{q}\right)-\mathfrak{R}^{N}\left(\mathbf{Q}^{N}\right)\right]=\mathscr{T}^{N},
$$

where it becomes evident that the truncation error acts as the source/production term of the discretization error.

\subsubsection{New Formulation}

A new formulation of the truncation error can be obtained by redefining the discrete and continuous partial differential operators, while still being consistent with the definition of the truncation error (10.1). Let us first redefine the discrete partial differentiation operator as

$$
\tilde{\mathfrak{R}}^{N}=\underline{\mathbf{M}}^{-1} \mathfrak{R}^{N}\left(\mathbf{Q}^{N}\right) .
$$

In the DGSEM, (10.8) leads directly to the point-wise definition,

$$
\tilde{\mathfrak{r}}_{j}^{N}\left(\mathbf{I}^{N}(\mathbf{q})\right)=\frac{1}{J_{j} w_{j}}\left[\int_{\partial \Omega^{e}}^{N} \hat{\mathbf{f}}\left(\mathbf{I}^{N}(\mathbf{q}), \mathbf{I}^{N}(\mathbf{q}), \vec{n}\right) \phi_{j} \mathrm{~d} S .-\int_{\Omega^{e}}^{N} \stackrel{\leftrightarrow}{\mathbf{f}}\left(\mathbf{I}^{N}(\mathbf{q})\right) \cdot \vec{\nabla} \phi_{j} \mathrm{~d} \Omega\right]-\mathbf{s}^{N}
$$

where $J_{j}$ is the point-wise determinant of the mapping on the node $j$ and $w_{j}$ is the corresponding quadrature weight. As a result, the sampled form of the discretized equation is

$$
\frac{\mathrm{d} \mathbf{Q}^{N}}{\mathrm{~d} t}+\tilde{\mathfrak{R}}^{N}\left(\mathbf{Q}^{N}\right)=\mathbf{0} .
$$

With this definition of the discrete partial differential operator, a consistent definition of the continuous partial differentiation operator is simply the strong form of the PDE,

$$
\tilde{\mathfrak{r}}(\mathbf{q})=\vec{\nabla} \cdot \overleftrightarrow{\mathbf{f}}-\mathbf{s}
$$


which can also be written in sampled form as

$$
\frac{\mathrm{d} \mathbf{Q}}{\mathrm{d} t}+\tilde{\mathfrak{R}}(\mathbf{Q})=\mathbf{0}
$$

Using (10.1), (10.10) and (10.12), we obtain a new sampled form of the DETE,

$$
\frac{\partial \mathscr{E}}{\partial t}+\left[\tilde{\mathfrak{R}}^{N}\left(\boldsymbol{I}^{N} \mathbf{q}\right)-\tilde{\mathfrak{R}}^{N}\left(\mathbf{Q}^{N}\right)\right]=\tilde{\mathscr{T}}^{N}
$$

where the new definition of the truncation error is simply,

$$
\tilde{\mathscr{T}}^{N}=\underline{\mathbf{M}}^{-1} \mathscr{T}^{N}
$$

We have derived two formulations that rely on the weak or strong forms of the PDE, and showed that each leads to a different version of the DETE. Although both formulations are apparently similar, a few remarks can be made about their properties.

1. The traditional version of the truncation error acts as a source term for the discretization error, after projecting it point-wise on the basis functions, $\phi_{j}$, that build the finite element subspace $\mathscr{V}^{N}$. On the other hand, the new approximation of the truncation error acts directly as a source term of the point-wise values of the discretization error.

2. Since the DGSEM is a collocation method and the mass matrix is a diagonal matrix containing the mapping Jacobian and quadrature weights, as seen in Section 2.2.1, each form of the truncation error can be obtained from the other by scaling it point-wise with $J_{j} w_{j}$. In other words, the main difference between both formulations is the weight that they give to the element size.

3. Because of the strong similarities between the two truncation error approximations, the anisotropic properties analyzed in Chapter 8 and the possibility to estimate the error in a multigrid cycle (Chapter 9) hold for both error measures. In fact, the new formulation of the truncation error can be directly obtained from the coarse-grid source term of the multigrid method (4.13) without scaling with the mass matrix terms.

Due to the similarities of both truncation error formulations, the tilde notation will be dropped in next sections, and the expressions will hold for both, unless the contrary is explicitly stated. A complete analysis of the differences between the two forms of the truncation error is out of the scope of this thesis and is left for future investigations. 


\subsection{Truncation Error Estimation in Unsteady Problems}

Now that we have a definition of the truncation error that is suitable for unsteady problems, we need a method to estimate the error when the exact solution is not available. In previous chapters, we considered steady problems, where only the first term of (10.1) takes nonzero values. We approximated that term with the steadystate version of the $\tau$-estimation method, using the solution on a high-order mesh $(P>N)$,

$$
\mathfrak{r}^{N}\left(\mathbf{I}^{N}(\mathbf{q})\right) \approx \mathfrak{r}^{N}\left(\mathbf{I}^{N}\left(\mathbf{q}^{P}\right)\right)
$$

In unsteady problems, we can either estimate the second term of (10.1) or reduce the problem to a steady-state case. The two main alternatives are listed below.

1. Variational DG form: To estimate the second term of (10.1), it is also reasonable to rely on the high-order solution, $\mathbf{q}^{P}$, and to approximate the continuous partial differential operator by the high-order one, $\mathfrak{r}^{P}$, in order to obtain

$$
\mathfrak{r}(\mathbf{q}) \approx \mathfrak{r}^{P}\left(\mathbf{q}^{P}\right)
$$

2. Dual time-stepping: The original PDE can be reformulated using a dual timestepping technique as $[8,181]$,

$$
\frac{\partial \mathbf{q}}{\partial \tau}+\frac{\partial \mathbf{q}}{\partial t}+\vec{\nabla} \cdot \stackrel{\leftrightarrow}{\mathbf{f}}=\mathbf{s}
$$

where $\tau$ is a pseudo-time that is marched to steady state in every time step of the physical time, $t$. In dual time-stepping methods, the time derivative is usually discretized with an implicit method and the pseudo-time derivative is either discretized with implicit or explicit methods.

The advantage of using an explicit scheme for the pseudo-time derivative is that the physical problem is integrated in time implicitly, without having to solve linear systems. All in all, the discretized/sampled system yields

$$
\underline{\mathbf{M}} \frac{\delta_{s}}{\delta \tau} \mathbf{Q}^{N}+\underline{\mathbf{M}} \frac{\delta_{p}}{\delta t} \mathbf{Q}^{N}+\mathbf{H}^{N}\left(\mathbf{Q}^{N}\right)=\underline{\mathbf{M}} \mathbf{S}^{N}
$$

where $\delta_{s} / \delta \tau$ is an operator that imposes the chosen pseudo-time-integration scheme and $\delta_{p} / \delta t$ is the operator for the chosen time-integration scheme.

Since we now have a steady problem, it is possible to consider only the first term of (10.1) if the partial differential operators are redefined as

$$
\mathfrak{R}^{N}\left(\mathbf{Q}^{N}\right)=\underline{\mathbf{M}} \frac{\delta_{p}}{\delta t} \mathbf{Q}^{N}+\mathbf{H}^{N}\left(\mathbf{Q}^{N}\right)-\underline{\mathbf{M}} \mathbf{S}^{N}
$$


for the traditional formulation, and

$$
\tilde{\mathfrak{R}}^{N}\left(\mathbf{Q}^{N}\right)=\frac{\delta_{p}}{\delta t} \mathbf{Q}^{N}+\underline{\mathbf{M}}^{-1} \mathbf{H}^{N}\left(\mathbf{Q}^{N}\right)-\mathbf{S}^{N}
$$

for the new formulation.

One could also be tempted to approximate q directly by the local (elemental) value of $\mathbf{q}^{P}$, substitute it in the definition of $\mathfrak{r}$, which is physics-dependent, and compute the derivatives and other operations locally and symbolically to approximate the second term of $(10.1)$ as $\mathfrak{r}(\mathbf{q}) \approx \mathfrak{r}\left(\mathbf{q}^{P}\right)$. However, this procedure would be equivalent to the variational DG approach, and would necessarily yield the isolated truncation error estimation (only local operations are taken into account). Therefore, it would not always be consistent with the definition of $\mathfrak{r}^{N}$.

In the rest of this chapter, we use the variational DG form of the unsteady truncation error since it is obtained directly from the fine-grid solution. The operator $\mathfrak{r}^{P}\left(\mathbf{q}^{P}\right)$ is taken as the isolated or non-isolated truncation error, so that the expression is consistent with the first term of (10.1) .

As a final remark, note that if a multigrid method is used to estimate the truncation error, the truncation error obtained with the variational DG form can be computed directly from the coarse-grid source term in (4.13).

\section{4 -Adaptation Strategies}

Two adaptation strategies can be identified in unsteady flow simulations: dynamic and static adaptation. These two strategies have already been widely used for unsteady adaptivity. See for example [25, 38] for dynamic adaptation methods, or $[67,150]$ for static adaptation methods.

In the following sections, we present a detailed description of how dynamic and static $p$-adaptation can be implemented for $\tau$-based $p$-adaptation methods. The main difference between the strategies presented here and those encountered in the literature is the way the adaptation algorithms treat the error estimates.

Most error estimation strategies available in the literature are designed to mark a number of the elements for enrichment or order reduction [25, 38, 150]. As a result, in every adaptation stage the polynomial orders are increased or reduced by one. On the contrary, the truncation error estimation provides an exact value of the needed polynomial order for each coordinate direction of every element after each estimation stage. This property of the $\tau$-estimation method, which is discussed in more detail in Section 7.2, provides several advantages for the $p$-adaptation of unsteady computations, as will be discussed in what follows. 


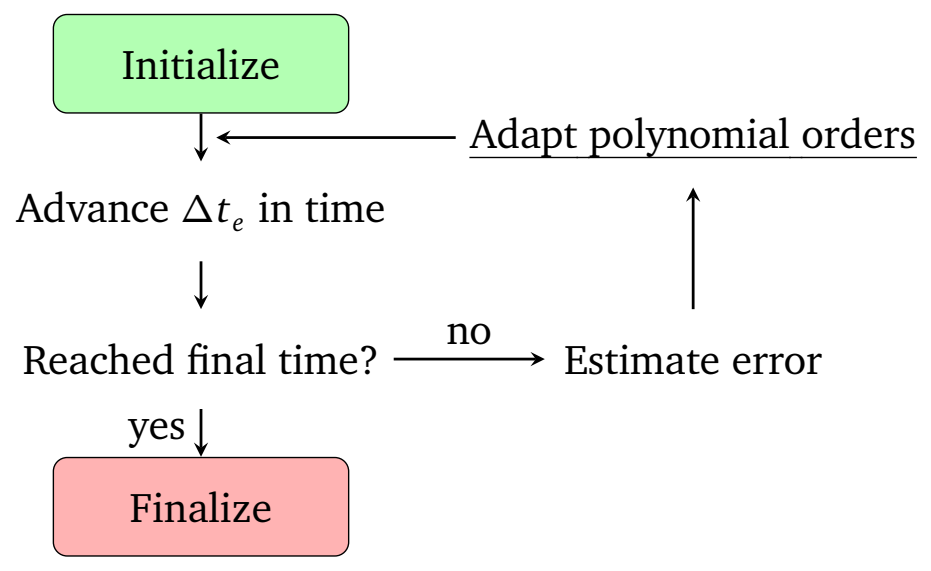

Figure 10.1: Flowchart of the dynamic p-adaptation.

\subsubsection{Dynamic $p$-Adaptation}

Dynamic $p$-adaptation is the most straight-forward $p$-adaptation strategy for unsteady flows. It computes an error measure periodically during a simulation (e.g. with the concepts of Section 10.3), and adapts the polynomial orders of the discretization according to the estimated error (e.g. with the method of Section 8.3.3), right after every estimation procedure.

Figure 10.1 illustrates the dynamic $p$-adaptation process. The interval between adaptation stages, $\Delta t_{e}$, can be specified as a physical time, as a number of iterations (time steps), or can be changed throughout the simulation. Every time a $p$-adaptation stage, the underlined process, is performed, the storage must be reallocated and the solution projected to the new polynomial spaces. Since that process is done several times during the solution procedure, overhead is expected. Therefore, the construction of the data structures for the new spatial resolution and the transfer of information are critical steps that must be optimized to enhance the performance. Furthermore, if the $\tau$-estimation method is used, a number of low-order $(N<P)$ discretizations are needed to evaluate the truncation error. As a result, an extra overhead is added in the construction of these coarse grids.

Note that a traditional error estimator, which simply marks some elements for refinement or coarsening, may perform poorly with the dynamic $p$-adaptation strategy of Figure 10. Such an error estimator imposes a one-by-one increase in the polynomial order. Therefore, if $\Delta t_{e}$ is too large, a dynamic $p$-adaptation strategy may not have enough time to increase the resolution of a zone of the domain before the flow feature of interest goes out of it. In other words, the refinement zones are likely to lag behind the difficult-to-capture flow features. On the contrary, since the truncation error estimator identifies what polynomial order is needed immediately, the resolution can be increased to the necessary level right away. As a result, the truncation error estimator may be more suitable to handle larger values of $\Delta t_{e}$ than traditional estimators. 
As was shown in Section 9.5.1.3, it is possible to obtain overshoots in the truncation error estimates if the reference mesh polynomial order, $P$, is too low, as illustrated in Figure 9.7. Therefore, a $\tau$-based dynamic $p$-adaptation method may suffer unneeded polynomial order oscillations that are caused, and nurtured, by the constant jump between a low and a high $P$. These polynomial order oscillations may deteriorate the accuracy and, therefore, should be avoided when possible. A possible way to attenuate this phenomenon is to limit the maximum polynomial order jump (by element and coordinate direction) after each $p$-adaptation stage.

Additionally, in parallelized simulations, a dynamic $p$-adaptation strategy requires dynamic load balancing to maintain an even workload between the processors and avoid deadlocks. Otherwise, the reduction in the number of degrees of freedom that is achieved with the enhanced spatial discretization may not translate into shorter computation times. The design of efficient dynamic load balancing algorithms is a challenging topic of research that is not treated in this work.

\subsubsection{Static $p$-Adaptation}

The static $p$-adaptation strategy differs from the dynamic $p$-adaptation in that only one $p$-adaptation process is performed. Figure 10.2 presents a flowchart of the static adaptation strategy. First, the solution is advanced in time with a fixed spatial resolution until a final estimation time, $T_{e}$, is reached. During this stage, periodic error estimations are performed with an interval of $\Delta t_{e}$ but, instead of changing the spatial resolution immediately, the error estimation is stored for future processing. When $T_{e}$ is reached, a $p$-adaptation procedure is performed using all the stored error estimates. Subsequently, the simulation is advanced in time with the new fixed spatial resolution until the final time.

Note that a traditional error estimator, which only marks some elements for oneby-one refinement or coarsening, may also perform poorly in the static $p$-adaptation algorithm of Figure 10.2. As a matter of fact, if such an error estimator is used, the algorithm would have to be slightly modified, so that after the $p$-adaptation stage the simulation goes back to the error estimation stage, as in [150]. That extra loop would have to be repeated a specific number of times, or until no element is marked for refinement or coarsening., which represents increased computational cost. Therefore, the ability to predict the exact polynomial that is needed makes the truncation error estimator an attractive indicator for statically $p$-adapted unsteady simulations.

The static $p$-adaptation strategy provides several implementation advantages over the dynamic $p$-adaptation. First, the construction of the data structures and the projection of the solution to the new spatial discretization are no longer critical steps as the $p$-adaptation procedure is only done once. Therefore, these operations can even be performed off-line and their computational cost does not significantly impact the performance of the method. Second, the coarse-grid discretizations that are needed for the truncation error estimation are only constructed once at 


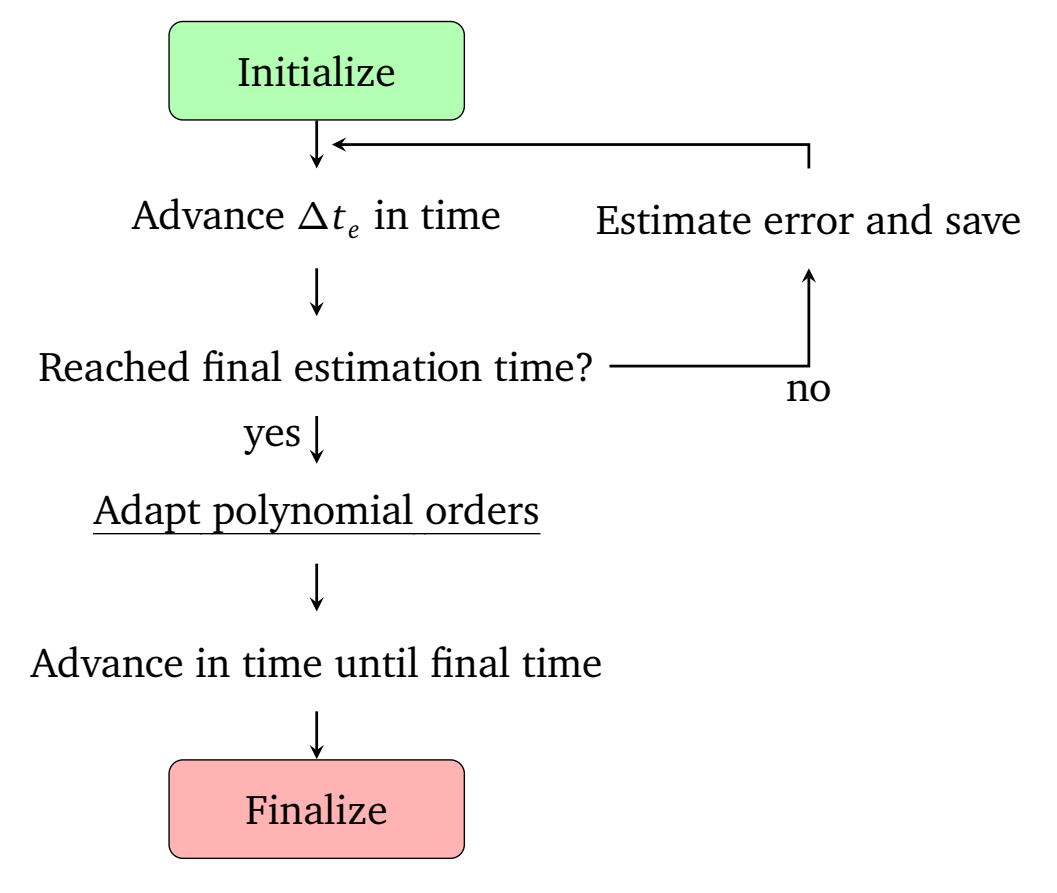

Figure 10.2: Flowchart of the static $p$-adaptation.

the beginning of the simulation and used throughout the whole estimation stage. Finally, dynamic load balancing is no longer needed since the loads must only be balanced once after the $p$-adaptation step.

The static $p$-adaptation algorithm has two drawbacks. First, it is only useful for statistically steady flows, or where the features that need high spatial resolution are located in a specific region of the domain. If the flow features move through a large part of the domain, dynamic $p$-adaptation probably yields better results. Second, the static $p$-adaptation strategy requires a preliminary simulation to estimate the error.

In the case of aerodynamic simulations of external flow, the flows are usually statistically steady and the interesting flow features are concentrated in a small region of the domain. Moreover, the estimation time is generally much shorter than the total simulation time.

To process the $p$-anisotropic truncation error estimation data and feed the $p$ adaptation algorithm, two main approaches can be identified.

1. At each estimation stage, $s$, select the polynomial orders for each of the elements in the mesh, $N_{i}^{e, s}$, and then predict a final polynomial order from the estimates,

$$
N_{i}^{e}=F\left(N_{i}^{e, 1}, \ldots, N_{i}^{e, n_{e}}\right),
$$

where $n_{e}$ is the number of estimation stages.

2. At each estimation stage, $s$, generate the truncation error map for every element of the mesh, $\left\|\tau^{N_{1} N_{2}}\right\|_{\infty}^{e, s}$, compute a total truncation error map for every 
element based on the estimations,

$$
\left\|\tau^{N_{1} N_{2}}\right\|_{\infty}^{e}=F\left(\left\|\tau^{N_{1} N_{2}}\right\|_{\infty}^{e, 1}, \ldots,\left\|\tau^{N_{1} N_{2}}\right\|_{\infty}^{e, n_{e}}\right)
$$

and select the polynomial order from the total truncation error map.

The function $F(\cdot)$ can be defined in multiple ways, for example the average or maximum functions,

$$
F_{\mathrm{av}}\left(N_{i}^{e, 1}, \ldots, N_{i}^{e, s}\right)=\frac{1}{n_{e}} \sum_{s=1}^{n_{e}} N_{i}^{e, s}, \quad F_{\max }\left(N_{i}^{e, 1}, \ldots, N_{i}^{e, s}\right)=\max _{s}\left|N_{i}^{e, s}\right| .
$$

As a conservative criterion, we use $F_{\max }$ to guarantee that the specified truncation error threshold is satisfied throughout the whole simulation.

Approach 1 needs less storage space and can be implemented more easily than approach 2. However, it may lead to the over-enrichment of some areas of the domain when combined with $p$-anisotropic discretizations. To illustrate this, let us consider a specific element in a hypothetical two-stage estimation procedure $\left(n_{e}=2\right)$ of a 2D simulation that uses $F_{\max }$. Let us assume $N_{\min }=1, N_{\max }=3$, and use the algorithm of Section 9.3, which selects the polynomial order combination that minimizes the number of degrees of freedom (NDOF).

Table 10.1 shows a possible outcome of the two-stage estimation procedure. There are five polynomial orders that fulfill the specified error threshold, $\tau_{\max }$, in each estimation stage. Among those, approach 1 would select the red underlined polynomial orders in each estimation stage because they minimize the instant NDOF. As can be observed, at the end of the estimation, approach 1 selects the polynomial orders $N^{e}=(3,3)$, that correspond to NDOF $=16$. This outcome is not optimal since $N^{e}=(2,2)$, with an associated $\mathrm{NDOF}=8$, would actually fulfill $\tau_{\max }$ with fewer degrees of freedom.

\begin{tabular}{|c|c|c|c|c|c|c|c|c|c|c|}
\hline \multirow[t]{2}{*}{$\begin{array}{c}\text { Coordinate } \\
\text { direction } \\
i \\
1\end{array}$} & & $\begin{array}{l}\text { lyn } \\
\text { Sta }\end{array}$ & nia & $\begin{array}{c}\text { orc } \\
1\end{array}$ & ers & vith & \multicolumn{3}{|c|}{$\begin{array}{c}\left\|\tau^{N}\right\|_{\infty}<\tau_{\max } \\
\text { Stage } s=2 \\
N_{i}^{e, 2}\end{array}$} & $\begin{array}{c}\text { Selected } \\
\text { order } \\
N_{i}^{e} \\
\end{array}$ \\
\hline & 3 & 2 & 3 & 2 & 1 & 3 & 2 & 3 & & 3 \\
\hline 2 & 3 & 3 & 2 & 2 & $\overline{3}$ & 3 & 3 & 2 & 2 & 3 \\
\hline NDOF & 16 & 12 & 12 & 9 & 8 & 16 & 12 & 12 & 9 & 16 \\
\hline
\end{tabular}

Table 10.1: Possible outcome of the approach 1 to static $p$-adaptation.

Approach 2 generates a total truncation error map by applying $F_{\max }$. The use of $F_{\max }$ implies that the polynomial order combinations that fulfill $\tau_{\max }$ in the total map are the ones that fulfill $\tau_{\max }$ in all the estimation stages, i.e. the combinations that are not red underlined. Out of that set of combinations, approach 2 clearly selects $N^{e}=(2,2)$, with an associated $\mathrm{NDOF}=8$. 
As was shown in this simple example, approach 2 is better than approach 1 . Therefore, approach 2 is selected for the static $p$-adaptation simulations that are shown in this chapter.

In spite of being superior, approach 2 has two drawbacks. First and foremost, the extrapolated truncation error map must be obtained for each estimation process, so that the total truncation error map can be obtained with (10.22). This requires a slight modification to the algorithm of Section 9.3 and more computational resources per estimation stage. Second, if an element is not in the asymptotic range in any of its reference coordinate directions ${ }^{1}$, it may not be possible to extrapolate the values of the inner truncation error map. In such cases, instead of extrapolating the directional truncation error, $\tau_{i}$, a very high value is assigned to it for $N_{i} \geq P_{i}$ as a secure criterion.

\subsection{Numerical Results}

In this section, we test the performance of the methods described in this chapter to perform $p$-adaptation of unsteady flow problems using truncation error estimates. Because of the superiority of the isolated truncation error, it is employed in this section to drive the $p$-adaptation procedures.

The time marching scheme in following examples is the Williamson's low-storage third order Runge-Kutta method (Section 3.2.1). Additionally, the time-step size is changed dynamically using the CFL condition in all simulations, as explained in Section 3.2.2. The main reason is that we want to take as large time steps as possible, and the time-step size is a function, among others, of the polynomial order. Furthermore, since the flow features that are analyzed have a periodicity in time, the interval between estimation/adaptation stages, $\Delta t_{e}$, is selected as a constant time for each simulation, and not as the time that corresponds to a number of time steps.

\subsubsection{Advection of a Vortex in a Uniform Flow}

In this section, the advection of a Gaussian vortex flowing in a square domain with periodic boundary conditions is simulated with the compressible Euler equations of gas dynamics and $\mathrm{Ma}_{\infty}=0.5$. The initial condition is prescribed as

$$
\begin{array}{ll}
u=1 & \rho=e^{5\left(x^{2}+y^{2}\right)}+1 \\
v=0 & p=1
\end{array}
$$

and a 2D structured mesh of 841 quadrilateral elements is used, as shown in Figure 10.3. The simulation is stopped at $t=29$, at the time where the vortex should be back were it started.

\footnotetext{
${ }^{1}$ As was discussed in Section 8.3.5, a positive slope in the estimation or a low correlation coefficient indicates that an element is not in the asymptotic range.
} 


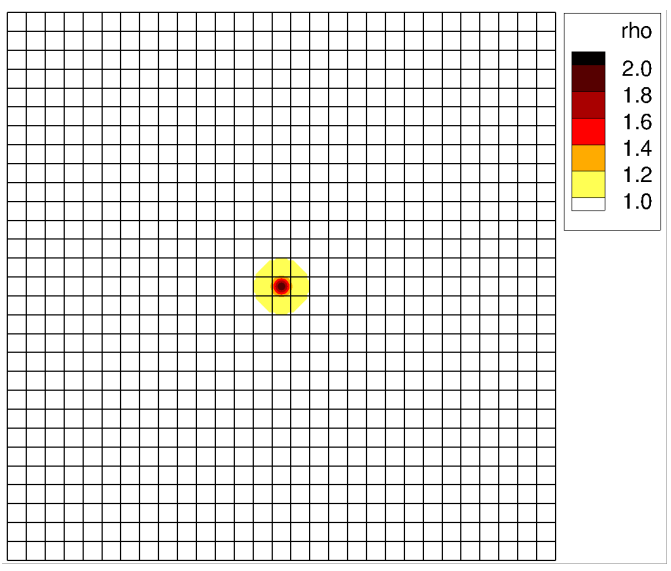

Figure 10.3: Initial condition of the flowing vortex simulation.

In this test case, both forms of the truncation error (traditional and new) perform equivalently. Namely, the polynomial order distributions that are obtained with the new formulation of the truncation error are almost identical to the ones obtained with the traditional formulation, if the specified error threshold, $\tau_{\max }$, is scaled with the (constant) element size. As concluded in Section 10.2, the main difference between the two formulations is that the traditional truncation error is scaled with the element size, and, since the size is uniform in the whole domain, no significant difference is observed between using one or the other. Following results are reported with the traditional isolated truncation error.

Both the static and the dynamic $p$-adaptation algorithms are tested with truncation error thresholds ranging between $10^{-3} \leq \hat{\tau}_{\text {max }} \leq 1$, and intervals between adaptation/estimation stages ranging between $0.5 \leq \Delta t_{e} \leq 10$. The polynomial order is adapted according to the output of the error estimations in the range $1 \leq N_{i} \leq 8$ for each direction $i$ of any element. Furthermore, the two different polynomial order jump conditions that were introduced in Section 9.5 are considered:

(a) The first one imposes that the polynomial order after every adaptation stage must fulfill

$$
N_{i}^{+} \geq\left\lfloor\frac{2}{3} N_{i}^{-}\right\rfloor
$$

where $N_{i}^{+}$is the polynomial order of the element in the coordinate direction $i$, and $N_{i}^{-}$is the polynomial order of a neighbor element in the matching coordinate direction $i$.

(b) The second polynomial order jump condition imposes

$$
N_{i}^{+} \geq\left\lfloor N_{i}^{-}-1\right\rfloor
$$




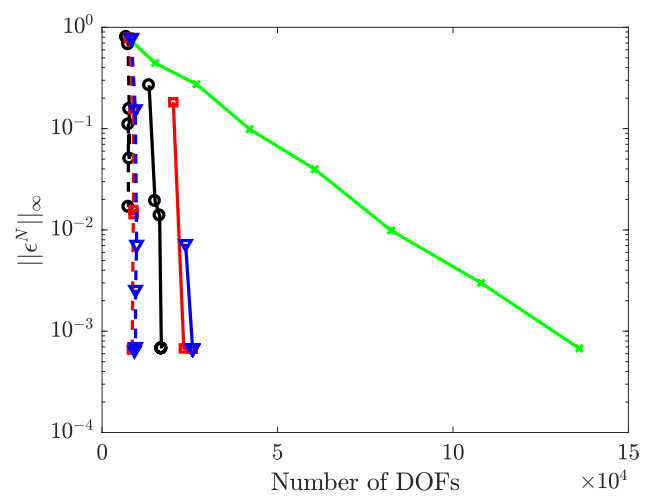

(a) Maximum error vs. number of DOFs.

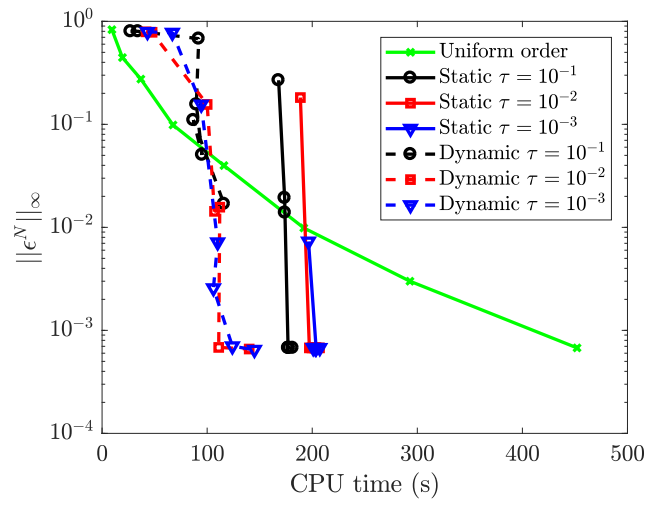

(b) Maximum error vs. CPU-Time.

Figure 10.4: Error performance of the static and dynamic $p$-adaptation procedures for a polynomial order jump codition of $N_{i}^{+} \geq\left\lfloor 2 N_{i}^{-} / 3\right\rfloor$.

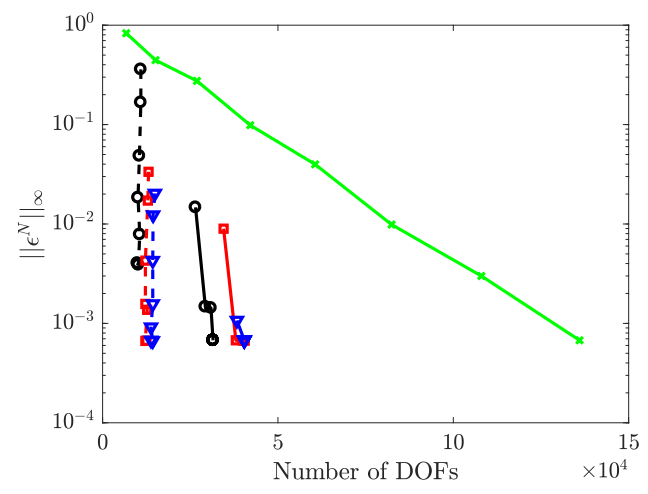

(a) Maximum error vs. number of DOFs.

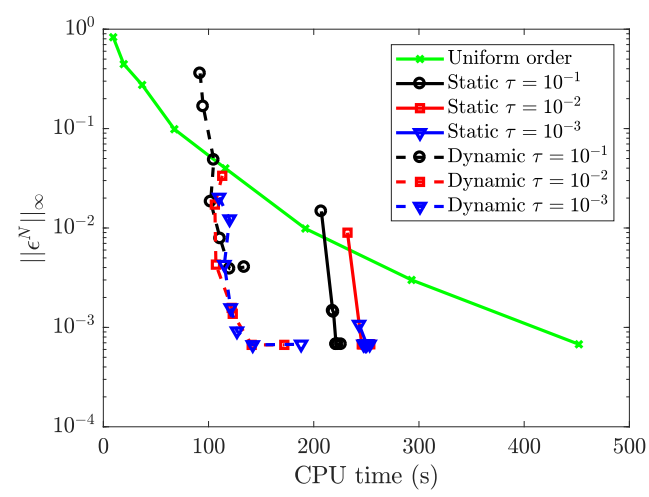

(b) Maximum error vs. CPU-Time.

Figure 10.5: Error performance of the static and dynamic $p$-adaptation procedures for a polynomial order jump codition of $N_{i}^{+} \geq\left\lfloor N_{i}^{-}-1\right\rfloor$.

In the static $p$-adaptation cases, a preliminary simulation must be run to estimate the error, as seen in Figure 10.2. Since the vortex is always changing its position, the preliminary simulation must be run for 29 time units to obtain a significant sample. To have enough points to extrapolate the anisotropic truncation error estimates, the $\tau$-estimation simulation uses a discretization of uniform order $P=3$.

All simulations are run in serial with a sixth generation 8-core intel i7 processor and 32GB of RAM, and the Roe solver is used as the advective numerical flux.

Figures 10.4 and 10.5 show the behavior of the dissipation error as a function of the number of the number of degrees of freedom and the computation time for the $p$-adaptive simulations with the polynomial order jump conditions of (10.25) 
and (10.26), respectively. The dissipation error is measured as the difference in $\rho$ between the exact solution and the simulation outcome at the centroid of the moving Gaussian. The dispersion error, which can be measured as the absolute value of the position of the Gaussian centroid, is of the order of machine zero (the DGSEM exhibits very low dispersion errors in this case). Note that the computation time needed for the estimation simulation in the static $p$-adaptation cases has already been added to the simulation time in Figures 10.4(b) and 10.5(b).

As can be observed, the $\tau$-based $p$-adaptation techniques perform better than the uniform refinement when a dissipation error $\left\|\epsilon^{N}\right\|_{\infty}<10^{-2}$ is desired, as they achieve the same errors with a fewer number of degrees of freedom, which translates into shorter computation times for a given accuracy.

Figures 10.4(a) and 10.5(a) show that the number of degrees of freedom for the statically $p$-adaptive simulations is $2-4$ times higher than for the dynamically $p$-adaptive simulation. This makes sense since the static $p$-adaptation algorithm enriches all the regions the vortex passes, whereas the dynamic $p$-adaptation algorithm effectively follows it. The longer computation times that are observed in Figures 10.4(b) and 10.5(b) for the statically $p$-adaptive simulations are not only the result of this effect, but also of the extra computation time that is invested for the preliminary $\tau$-estimation simulation.

The number of degrees of freedom of the $p$-adaptive simulations that obey the polynomial order jump condition (b) is higher than for the condition (a). This is expected since many more elements are enriched in the former, as can be observed in Figure 10.6. The extra enrichment translates to computation times up to $25 \%$ higher when using the condition (10.26).

An additional difference between the two polynomial order jump conditions, that can be inferred from Figure 10.6, is that the condition (a) is more sensitive to the estimation/adaptation interval, $\Delta t_{e}$. On the one hand, in the dynamically $p$-adaptive simulations and for a given $\Delta t_{e}$, it is more likely that the vortex escapes the refined area for the condition (a) than for (b), and arrives to an area where no $\tau$-estimation is possible $(P=1)$ or where no extrapolation is possible $(P<3)$. On the other hand, in the statically $p$-adaptive simulations and for a given $\Delta t_{e}$, the refinement areas are more likely to be connected if the condition (b) is used instead of (a). This behavior is also illustrated in Figure 10.7 for the static $p$-adaptation with the threshold $\hat{\tau}_{\max }=10^{-1}$.

\subsubsection{Subsonic Flow Past a Cylinder}

The flow around a circular cylinder at a Reynolds number of $\operatorname{Re}_{\infty}=100$ and a Mach number of $\mathrm{Ma}_{\infty}=0.15$ is simulated on a high-order curved $(M=3)$ mesh with 1282 quadrilateral elements and the DGSEM method. Several simulations are performed to assess the performance of the $\tau$-based static and dynamic $p$-adaptation methods, and of uniform refinement. All simulations use the Roe solver [170] as the advective numerical flux and BR1 [14] as the diffusive numerical flux. 


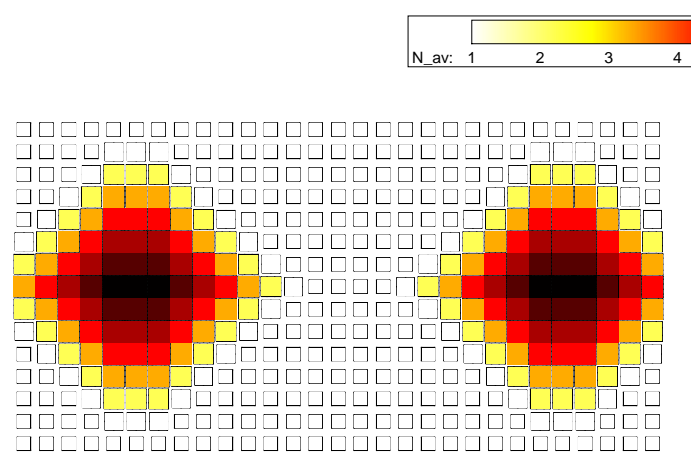

(a) $N_{i}^{+} \geq\left\lfloor N_{i}^{-}-1\right\rfloor, \Delta t_{e}=10$.

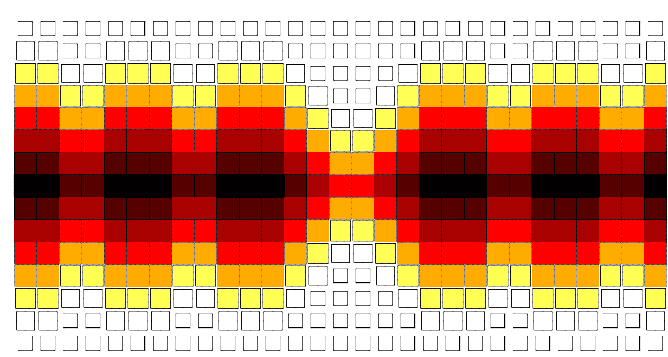

(c) $N_{i}^{+} \geq\left\lfloor N_{i}^{-}-1\right\rfloor, \Delta t_{e}=5$.

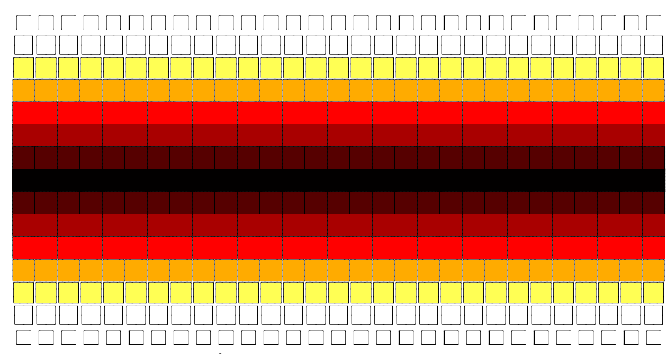

(e) $N_{i}^{+} \geq\left\lfloor N_{i}^{-}-1\right\rfloor, \Delta t_{e}=1$.

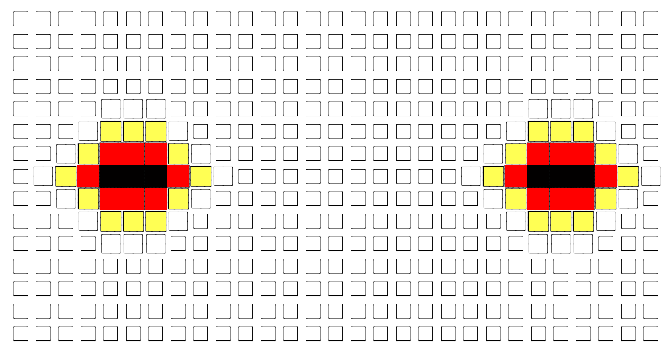

(b) $N_{i}^{+} \geq\left\lfloor 2 N_{i}^{-} / 3\right\rfloor, \Delta t_{e}=10$.

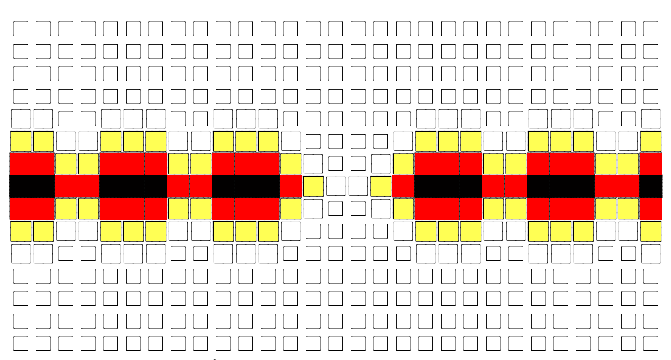

(d) $N_{i}^{+} \geq\left\lfloor 2 N_{i}^{-} / 3\right\rfloor, \Delta t_{e}=5$.

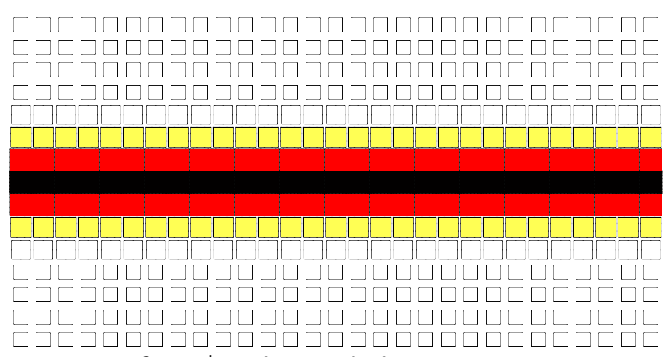

(f) $N_{i}^{+} \geq\left\lfloor 2 N_{i}^{-} / 3\right\rfloor, \Delta t_{e}=1$.

Figure 10.6: Average polynomial order distribution for static $p$-adaptation with $\tau_{\max }=10^{-1}$ and different estimation intervals.

Figure 10.8 shows the mesh that was used, the instantaneous horizontal velocity contours and an instantaneous distribution of polynomial orders for the dynamic $p$-adaptation method.

The results presented in this section were obtained using a 40-core $2.10 \mathrm{GHz}$ Intel(R) Xeon(R) Gold 6230 CPU with 170 GB of RAM. Each simulation was run with 10 cores and shared memory parallelization (OpenMP + guided schedule) to compute the spatial terms. Note that this parallel implementation has a near-optimal scalability for $p$-anisotropic discretizations and the selected OpenMP schedule, as can be seen in Appendix C.1. We remark that the guided OMP schedule acts directly as a dynamic load balancing technique in the simulations with dynamic $p$ adaptation.

For the $p$-adaptive simulations, the new form of the truncation error is retained 


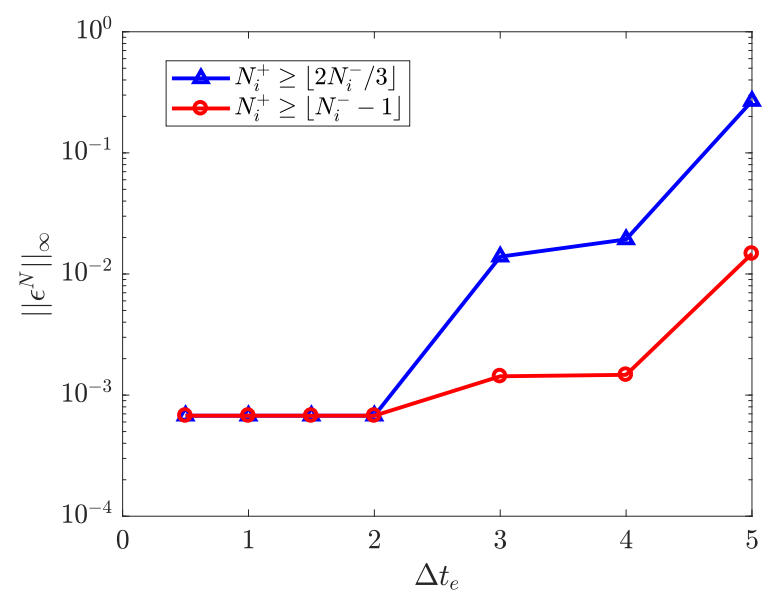

Figure 10.7: Combined effect of the polynomial order jump condition and the estimation interval on the error.

as it showed to work more efficiently on the lift and drag than the traditional form. The reason why is easily spotted in Figure 10.9, which shows the contours of the average polynomial orders for both formulations of the truncation error as the error threshold, $\tau_{\max }$, is reduced in a static $p$-adaptation method. For a similar number of degrees of freedom, the $p$-adaptation -algorithm that is uses the traditional form of $\tau$ tends to enrich large elements that are away from the cylinder, whereas the new form tends to enrich only the boundary layer area and the wake. As was explained in Section 10.2, the main difference between the two approaches is the weight they assign to the each element's volume.

The $\tau$-based static and dynamic $p$-adaptation algorithms are tested with truncation error thresholds ranging between $10^{-1} \leq \tilde{\tau}_{\max } \leq 10^{2}$, and four estimation/adaptation intervals $\Delta t_{e}=0.5,1,3,6$ in non-dimensional time units, taking into account that the vortex shedding period is $T=6$. Furthermore, the polynomial order jump across faces is limited to $N_{i}^{+} \geq\left\lfloor N_{i}^{-}-1\right\rfloor(10.26)$, since this condition provides robustness to the simulation, as discussed in Chapter 9, and allows larger estimation intervals, as discussed in Section 10.5.1. In addition, the maximum polynomial order was set to $N_{\max }=8$ and the minimum polynomial order to $N_{\min }=3$. This minimum polynomial order allows the dynamic $p$-adaptation to always have enough points to perform the directional truncation error extrapolation.

In the dynamic $p$-adaptation algorithm, the sub-meshes that are used for the truncation error estimation are constructed every $\Delta t_{e}$ time units. After that, the error is estimated using the $\tau$-estimation method explained in Chapter 8 and the polynomial orders are changed accordingly. The maximum polynomial order decrease is limited to one in each $p$-adaptation stage as a way to reduce the spurious oscillations that may arise because of large polynomial order jumps throughout the simulation, a phenomenon discussed in Section 10.4.1. In the static $p$-adaptation 


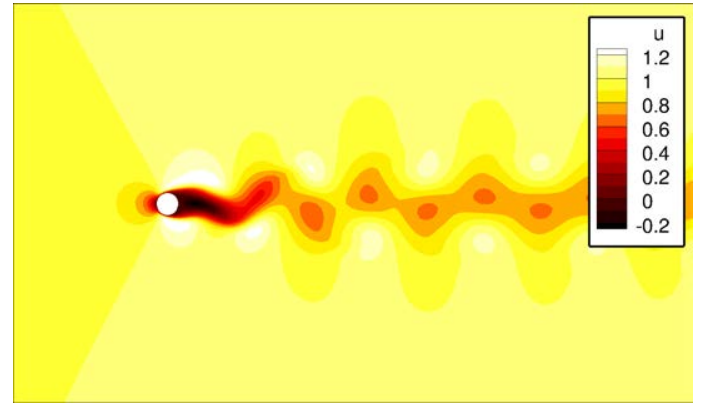

(a) Instant horizontal velocity contours.

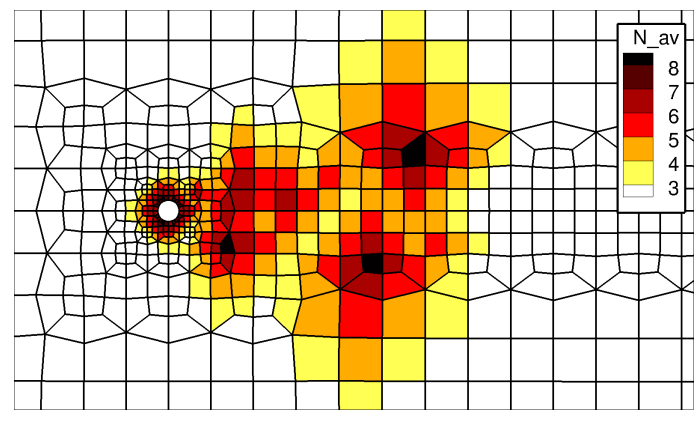

(b) Instant average polynomial orders (dynamic $p$-adaptation) for $\hat{\tilde{\tau}}_{\max }=5$.

Figure 10.8: Vortex shedding behind a cylinder at $\operatorname{Re}_{\infty}=100$.

algorithm, the $\tau$-estimation sub-meshes are only constructed once at the beginning of the simulation. Thereafter, an estimation simulation with polynomial order $P=4$ is run for a sampling time of $T_{e}=12$, i.e. two vortex shedding cycles. The polynomial orders are then adapted using strategy 2 (10.22), and the rest of the simulation is executed without further modifying them.

Figure 10.10 shows the performance of the uniform $p$-refinement, the dynamic and the static $\tau$-based $p$-adaptation algorithms. The mean absolute lift, the mean drag error and the Strouhal number error (the two latter with respect to a solution of order $N=9$ ) are plotted as a function of the number of degrees of freedom (NDOF) and the computation time for each of the methods. The lift and drag are monitored for 100 time units, and the mean and frequency values are extracted by applying a fast Fourier transform (FFT). In particular, the Strouhal number that is reported in Figures 10.10(e) and 10.10(f) is obtained from the lift curve. The reported computation time is the sum of the CPU-time that is needed to advance 100 time units, and the CPU-time that is needed for the estimation.

A Williamson's low-storage third-order Runge-Kutta method (RK3) is used as the time-marching scheme and the time-step size is dynamically adapted in each time step according to the advective and viscous CFL conditions, so that as large time steps as possible are taken. The adaptive time-stepping is an important feature since it favors a fair comparison of the computation times of discretizations with different polynomial orders. Because of the adaptive time-stepping, the monitored variables had to be resampled at a uniform time-step sequence to apply the FFT. In addition, the number of degrees of freedom that is shown for the dynamic $p$ adaptation simulations corresponds to a weighted average,

$$
\mathrm{NDOF}_{\mathrm{dyn}}=\frac{1}{S} \sum_{i=1}^{S} \mathrm{NDOF}_{i}
$$

where $S$ is the number of time steps of the simulation and $\mathrm{NDOF}_{i}$ corresponds to 


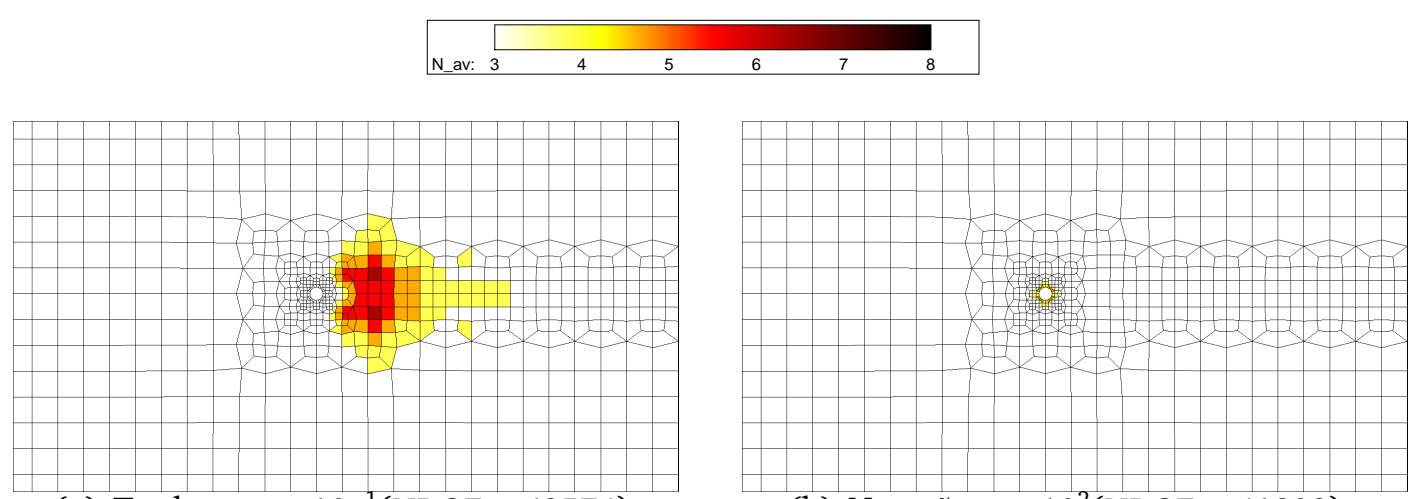

(a) Trad. $\tau_{\max }=10^{-1}(\mathrm{NDOF}=43574)$.

(b) New. $\tilde{\tau}_{\max }=10^{2}(\mathrm{NDOF}=41808)$.

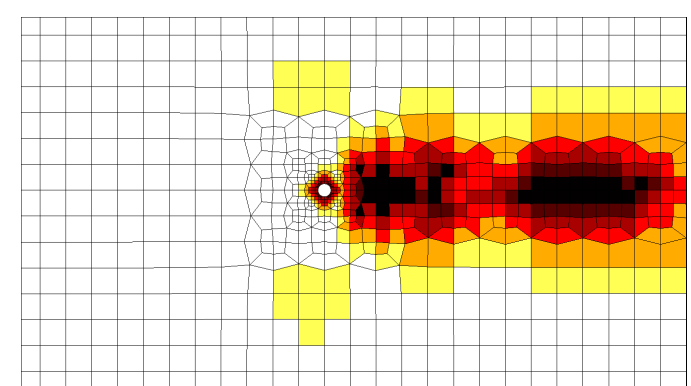

(c) Trad. $\tau_{\max }=10^{-2}(\mathrm{NDOF}=69644)$.

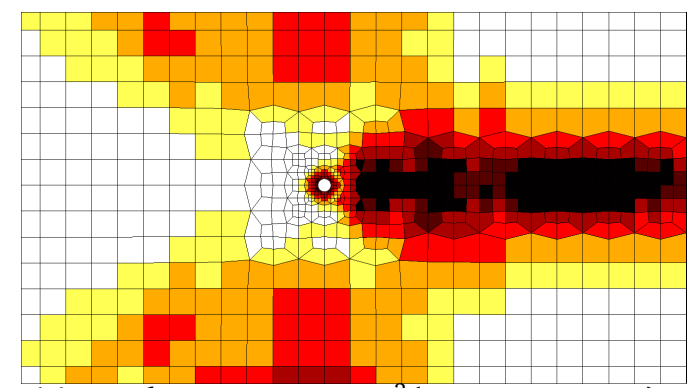

(e) Trad. $\tau_{\max }=5 \times 10^{-3}(\mathrm{NDOF}=90192)$.

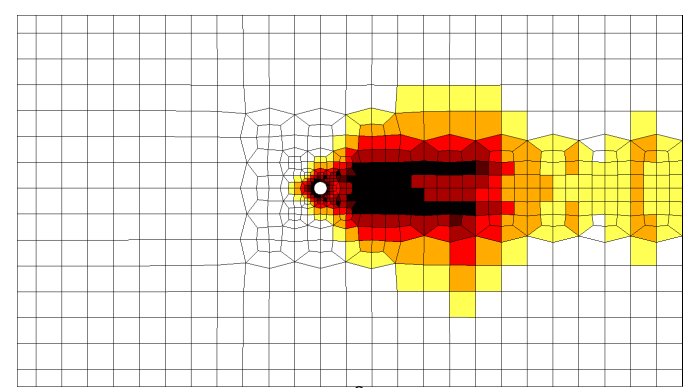

(d) New. $\tilde{\tau}_{\max }=10^{0}(\mathrm{NDOF}=67766)$.

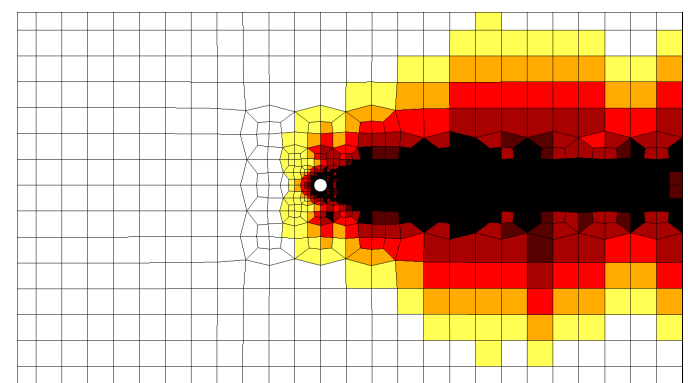

(f) New. $\tilde{\tau}_{\max }=10^{-1}(\mathrm{NDOF}=95930)$.

Figure 10.9: Comparison of the two possible formulations of the truncation error: traditional (left) and new (right). Average polynomial order distribution for static $p$-adaptation for different error thresholds.

the number of degrees of freedom of the discretization in the iteration $i$.

It can be observed that the $\tau$-based locally adaptive simulations need fewer degrees of freedom than the simulations with uniform order. Furthermore, as opposed to the advected vortex example, the static $p$-adaptation method needs fewer degrees of freedom than the dynamic $p$-adaptation method for the same levels of accuracy. The main reason for that behavior is the overestimation of the polynomial order needed when the reference mesh (estimation) polynomial order, $P$, is low (a behavior that was described in Section 9.5.1.3 and discussed in Section 
10.4.1). In fact, the dynamic $p$-adaptation algorithm is more likely to overpredict the polynomial order needed than the static algorithm since the minimum specified polynomial order sometimes acts as the estimation polynomial order in dynamically $p$-adaptive simulations, $P=N_{\min }=3$, which is lower than the estimation polynomial order of the static $p$-adaptation algorithm, $P=4$.

The behavior of the error with respect to the computation times is highly dependent on the implementation, the hardware that is employed and the problem. However, the results obtained with the current implementation in HORSES3D (Appendix C) are reported as a reference. As can be observed, the performance is different for each analyzed variable, but in general a speed-up of about 2.2 can be observed for the static $p$-adaptation algorithm at the highest level of accuracy that is reached. The dynamic $p$-adaptation algorithm has the same performance as the static $p$-adaptation algorithm in some cases, and in some others it exhibits a far worse performance. The main reason for that is that the dynamic $p$-adaptation algorithm is much more sensitive to the estimation interval, $\Delta t_{e}$, and that it may also suffer from non-physical oscillations in the solution and its gradients due to the frequent jumps in the polynomial order.

The $\tau$-based $p$-adaptation methods show the best performance when measuring the mean absolute lift, where speed-ups can be observed in virtually all the error range considered for small enough $\Delta t_{e}$. When measuring the mean drag error, $\tau$ based $p$-adaptation performs relatively similar to the uniform $p$-refinement (if $\Delta t_{e}$ is small enough) down to an error of $\left|\bar{C}_{d}-\bar{C}_{d}^{N=9}\right| \approx 5 \times 10^{-5}$. Below that error, the $\tau$-based $p$-adaptation algorithms outperform the uniform refinement technique. In contrast, the computational performance observed when measuring the Strouhal number is worse in the $\tau$-based $p$-adaptive cases than in uniform refinement for errors greater than $\left|\mathrm{St}_{d}-\mathrm{St}_{d}^{N=9}\right| \approx 5 \times 10^{-7}$. Below that error, the $\tau$-based $p$-adaptation algorithms outperform the uniform refinement technique once again. The main reason is that the error in the measured shedding frequency decreases very rapidly for low polynomial orders, where uniform refinement is still computationally more efficient than local $p$-adaptation, taking into account the overhead of estimating the error and changing the polynomial orders. This causes the $p$-adaptation methods to only show advantages for low errors in the Strouhal number, $\left|\mathrm{St}-\mathrm{St}^{N=9}\right|<4 \times 10^{-7}$.

\subsection{Final Remarks}

In this chapter, a study of $\tau$-based $p$-adaptation methods for unsteady flow problems is presented. The most relevant results of this study are:

1. The $\tau$-estimation method was extended to analyze the truncation error of unsteady flow problems with the DGSEM. The method developed here retains the anisotropic properties and the ability to be estimated in a multigrid cycle.

2. Two $\tau$-based $p$-adaptation strategies were proposed, the dynamic and the 
static adaptation methods. These strategies were analyzed and successfully used to enhance the performance of a DGSEM code.

3. The static $p$-adaptation method performs better than the dynamic one in statistically steady problems where the flow features are concentrated in a small part of the domain, as was verified in the cylinder test case. Similarly, the dynamic $p$-adaptation method outperforms the static one when the flow features move through a large portion of the domain, as was observed in the flowing vortex case.

4. A new form of the truncation error was presented, which holds close similarities to the one traditionally used in the literature for variational methods. This new form of the truncation error was shown to perform equally as to traditional form for a test case with uniform mesh size, and it was shown to yield better results than the traditional formulation for a selected test case with non-uniform mesh size.

Future research includes the evaluation of dual time-stepping methods, both as accelerators and as a way to estimate the truncation error for the $p$-adaptation method, and a detailed analysis of the differences between the traditional and new truncation error formulations. 


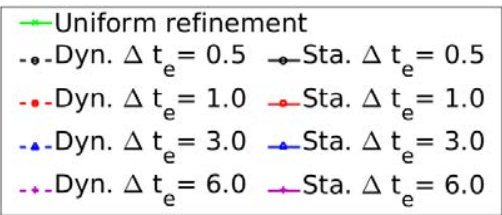

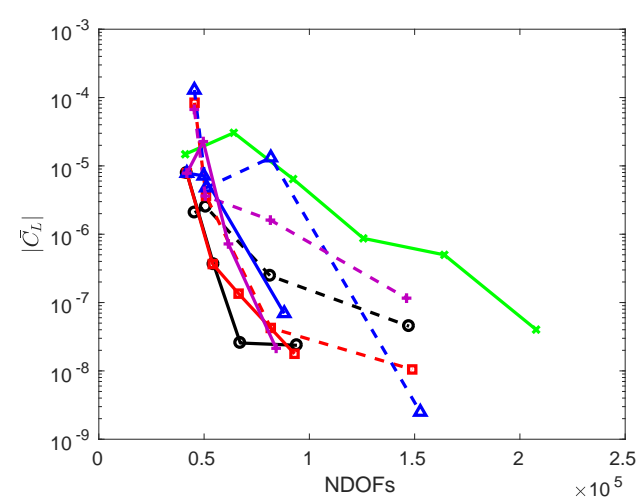

(a) Absolute lift vs. NDOF.

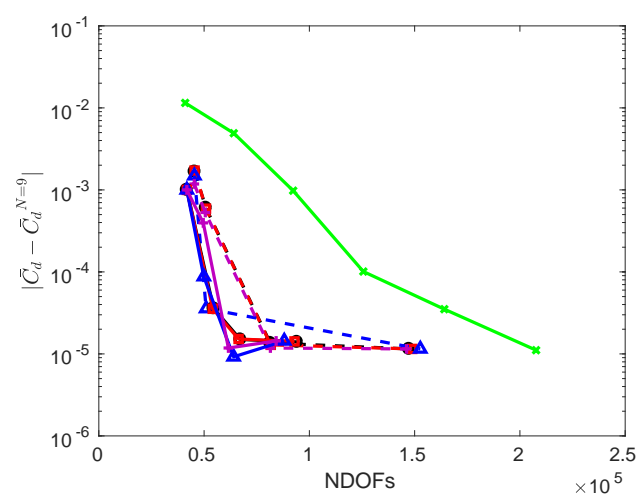

(c) Drag error vs. NDOF.

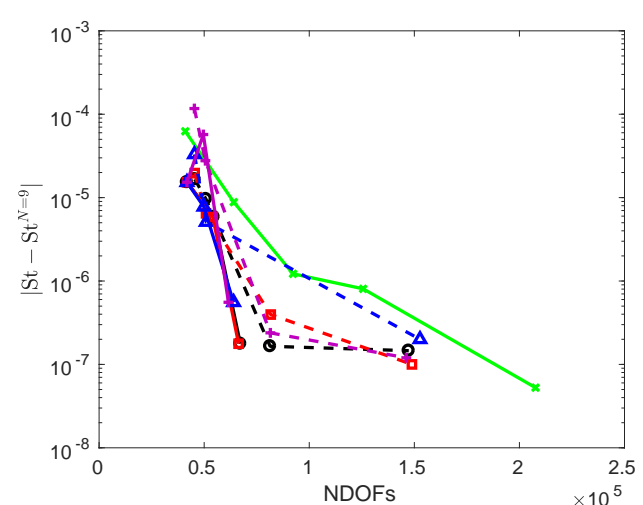

(e) Strouhal error vs. NDOF.

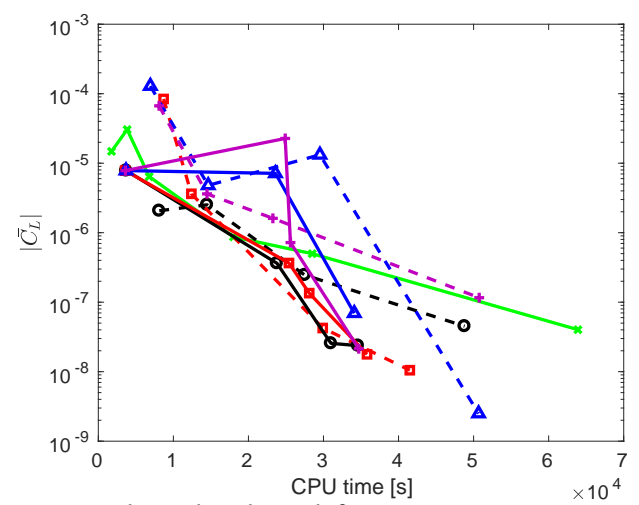

(b) Absolute lift vs. CPU-Time.

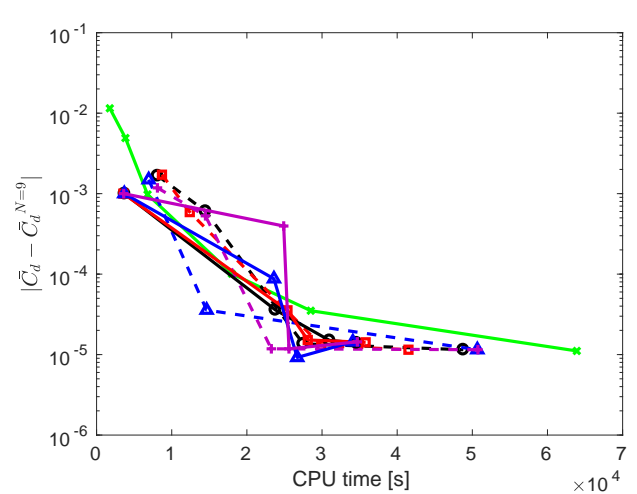

(d) Drag error vs. CPU-Time.

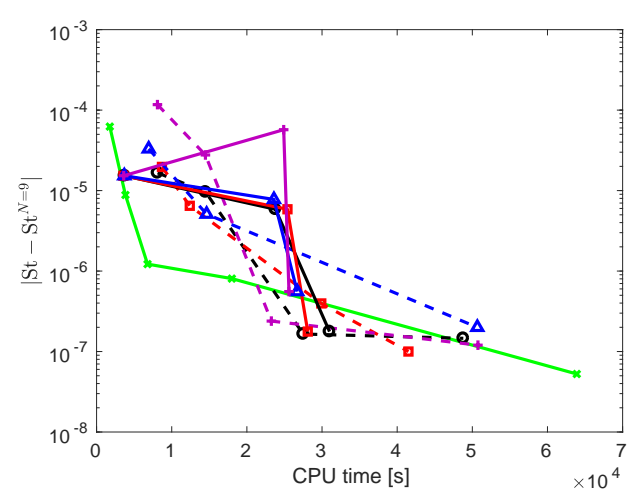

(f) Strouhal error vs. CPU-Time.

Figure 10.10: Performance of the static and dynamic $p$-adaptation procedures for the flow past a cylinder at $\operatorname{Re}_{\infty}=100, \mathrm{Ma}_{\infty}=0.15$. 


\section{Conclusions}

The results of this thesis have enhanced the computational efficiency of high-order discontinuous Galerkin (DG) methods for the solution of the time-dependent compressible Navier-Stokes equations. Two main areas were studied: (i) the improvement of the solution techniques that depend on the selected temporal discretization, with special emphasis on implicit time-integration methods and multigrid techniques, and (ii) the enhancement of the DG spatial discretization by means of local anisotropic p-adaptation strategies using truncation error estimations.

Regarding the implicit time-integration methods, the expressions for the DGSEM Jacobian matrix of the 3D compressible Navier-Stokes equations were derived and implemented numerically and analytically. In addition, it was shown that the DGSEM in Gauss-Lobatto points (GL-DGSEM) can be formulated as a Schur complement problem and solved using static condensation while keeping orthogonal basis functions, something that is not directly possible in other DG methods. Significant speed-ups are achieved using this technique when using moderate/high polynomial orders with both direct and Krylov subspace iterative methods, since the condensed systems are smaller in size and are better conditioned than the systems that are obtained with the original DG formulation.

With regard to the spatial discretization techniques, two main topics were analyzed: the formulation of an error estimator to drive mesh adaptation methods and the development of steady and unsteady anisotropic p-adaptation methods. First, a new truncation error estimator using the $\tau$-estimation method was developed for the DGSEM that is specifically designed to account for p-anisotropic representations that can have different polynomial orders in different directions of each element. This novel truncation error estimator was shown to be computationally cheaper and to yield a more accurate extrapolation of the estimated quantity than previous approaches that used the $\tau$-estimation method for the p-anisotropic DGSEM. Even though the method was developed and implemented for the DGSEM, it is applicable to any DG method with tensor-product basis functions.

The truncation error estimator was successfully used to perform local anisotropic p-adaptation in steady and unsteady flow problems. In steady-state problems, significant speed-ups were achieved by coupling the presented error estimator with an adaptation algorithm that minimizes the number of degrees of freedom for a desired accuracy, and a multigrid solver. For unsteady flow problems, two 
p-adaptation strategies were developed that use the new truncation error estimator: a static and a dynamic p-adaptation procedure. Both methods were shown to reduce the required number of degrees of freedom for a specific accuracy, being the latter the best choice in statistically steady flows.

Although the 3D compressible Navier-Stokes equations were studied in this work, the methods developed here can be directly extended to any advectiondiffusion equation, and easily to any PDE in conservative form.

\section{Future Challenges}

Ongoing work includes coupling the implicit time-integration methods with the p-adaptation strategies that were developed in this thesis. By doing this, further speed-ups are expected. A major challenge when doing dynamic p-adaptation is foreseen in the reallocation of the storage for the data structures of the implicit time-integration methods (Jacobian matrix and vectors), and in the dynamic load balancing. At the time of submission of this manuscript, the analytical Jacobian matrix is only implemented for p-conforming representations in HORSES3D.

The development of efficient preconditioners for DG is still an active research topic. On top of that, it was shown in Chapter 6 that a specific preconditioner can act very differently on the global linear system from a DG discretization than on the reduced-size linear system that results from applying static condensation. Consequently, an interesting field of research is to develop preconditioners that are specifically tailored for the statically-condensed systems.

Regarding the mesh adaptation strategies, future work includes a rigorous comparative analysis of the two truncation error formulations, new and traditional, and the development of hp- and hr-adaptation strategies that use truncation error estimations. As was discussed throughout the thesis, p-adaptation strategies are useful when the solution is smooth enough inside each element. As this is not always the case, the mesh adaptation strategy can be improved if certain elements of the mesh, where the solution is not smooth, are subdivided or shrunk. The truncation error estimator can be easily used to assess the smoothness of the solution, and therefore to mark where a reduction of the element size is advantageous to improve the discrete representation. The major challenge here is the implementation of efficient data structures for h-adaptation and dynamic load balancing techniques. 
Appendices 



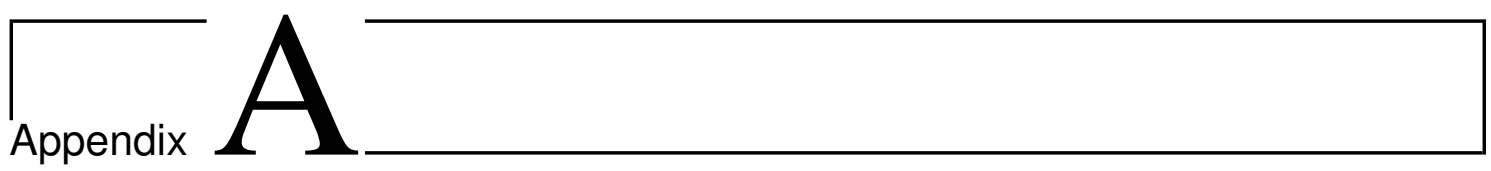

\section{Compressible Navier-Stokes Equations}

The compressible Navier-Stokes equations in conservative form can be written in nondimensional form as

$$
\partial_{t} \mathbf{q}+\vec{\nabla} \cdot\left(\overleftrightarrow{\mathbf{f}}^{a}-\overleftrightarrow{\mathbf{f}}^{v}\right)=\mathbf{s}
$$

where the conserved quantities are the mass, momentum and energy (per unit of volume), $\mathbf{q}=[\rho, \rho \vec{v}, \rho E]^{T}$, $\mathbf{s}$ is an external source term, and $\overleftrightarrow{\mathbf{f}}^{a}$ and $\overleftrightarrow{\mathbf{f}}^{v}$ are called the advective and diffusive flux tensors, respectively. The Euler equations of gas dynamics are defined in the same way, but omitting the viscous flux. The flux tensors can be written in compact form as

$$
\stackrel{\leftrightarrow}{\mathbf{f}}^{a}(\mathbf{q})=\left[\begin{array}{c}
\rho \vec{v} \\
\rho \vec{v} \otimes \vec{v}+\underline{\mathbf{I}} p \\
\vec{v}(\rho E+p)
\end{array}\right], \stackrel{\mathbf{f}}{ }^{v}(\mathbf{q}, \vec{\nabla} \mathbf{q})=\frac{1}{\operatorname{Re}_{\infty}}\left[\begin{array}{c}
\overrightarrow{0} \\
\underline{\tau} \\
\underline{\tau} \vec{v}+\kappa \vec{\nabla} T
\end{array}\right]
$$

Here, $\rho$ is the fluid's density, $\vec{v}$ is the velocity vector, $p$ is the (static) pressure, $E$ is specific the total energy (internal energy plus kinetic energy), $\tau$ is the stress tensor, $T$ is the temperature, $\operatorname{Re}_{\infty}$ is the reference Reynolds number, and $\kappa$ is a non-dimensional thermal conductivity.

The nondimensionalization is performed using reference values for the density $\left(\rho_{\infty}\right)$, velocity $\left(V_{\infty}\right)$, length $\left(L_{\infty}\right)$, dynamic viscosity $\left(\mu_{\infty}\right)$, and temperature $\left(T_{\infty}\right)$, which give the definition of the Reynolds number,

$$
\operatorname{Re}_{\infty}=V_{\infty} L_{\infty} \rho_{\infty} / \mu_{\infty}
$$

and of the nondimensional thermal conductivity,

$$
\kappa=\frac{\mu}{(\gamma-1) \operatorname{Pr} \mathrm{Ma}_{\infty}}
$$

where $\gamma=c_{p} / c_{v}$ is the heat capacity ratio, $c_{p}$ and $c_{v}$ are the heat capacity at constant pressure and constant volume, respectively, $\mathrm{Ma}_{\infty}=V_{\infty} / c_{\infty}$ is the reference Mach number, $c=\sqrt{\gamma p / \rho}$ is the speed of sound, $\operatorname{Pr}=c_{p} \mu / \kappa_{t h}$ is the Prandtl number (assumed to be constant), and $\kappa_{t h}$ is the thermal conductivity of the fluid. 
In external flows, as the ones considered in this thesis, the reference values are typically obtained from the far-field state, except from the reference length, which is a geometric characteristic length.

Furthermore, the pressure $p$ is computed using the calorically perfect gas approximation,

$$
p=(\gamma-1) \rho e,
$$

where $e=E-\|\vec{v}\|^{2} / 2$ is the specific internal energy, and the stress tensor is computed using the Stokes hypothesis,

$$
\underline{\tau}=\mu\left((\vec{\nabla} \vec{v})^{T}+\vec{\nabla} \vec{v}\right)-\lambda \vec{\nabla} \cdot \vec{v} \underline{\mathbf{I}}
$$

with $\lambda=-\frac{2}{3} \mu$ the bulk viscosity coefficient. In this thesis, we chose the typical parameters for air: $\operatorname{Pr}=0.72, \gamma=1.4$, while $\mu$ is calculated using Sutherland's law,

$$
\mu=\frac{1+T_{\text {suth }} / T_{\infty}}{T+T_{\text {suth }} / T_{\infty}} T^{\frac{3}{2}}
$$

where $T_{\text {suth }}=110.4 \mathrm{~K}$ is the Sutherland's temperature.

For three dimensional flows, $\vec{v}=[u, v, w]^{T}$, the compressible Navier-Stokes equations imply the conservation of $\mathbf{q}=[\rho, \rho u, \rho v, \rho w, \rho E]^{T}$ under the influence of the advective fluxes,

$$
\mathbf{f}_{1}^{a}=\left[\begin{array}{c}
\rho u \\
p+\rho u^{2} \\
\rho u v \\
\rho u w \\
u(\rho E+p)
\end{array}\right], \mathbf{f}_{2}^{a}=\left[\begin{array}{c}
\rho v \\
\rho u v \\
p+\rho v^{2} \\
\rho v w \\
v(\rho E+p)
\end{array}\right], \mathbf{f}_{3}^{a}=\left[\begin{array}{c}
\rho w \\
\rho u w \\
\rho v w \\
p+\rho w^{2} \\
w(\rho E+p)
\end{array}\right]
$$

and the diffusive fluxes,

$$
\mathbf{f}_{1}^{v}=\frac{1}{\operatorname{Re}_{\infty}}\left[\begin{array}{c}
0 \\
\tau_{x x} \\
\tau_{x y} \\
\tau_{x z} \\
v_{i} \tau_{1 i}+\kappa \partial_{x} T
\end{array}\right], \mathbf{f}_{2}^{v}=\frac{1}{\operatorname{Re}_{\infty}}\left[\begin{array}{c}
0 \\
\tau_{y x} \\
\tau_{y y} \\
\tau_{y z} \\
v_{i} \tau_{2 i}+\kappa \partial_{y} T
\end{array}\right], \mathbf{f}_{3}^{v}=\frac{1}{\operatorname{Re}_{\infty}}\left[\begin{array}{c}
0 \\
\tau_{z x} \\
\tau_{z y} \\
\tau_{z z} \\
v_{i} \tau_{3 i}+\kappa \partial_{z} T
\end{array}\right] .
$$

\section{A.1 Jacobians}

This section contains the flux Jacobians for the 3D compressible Navier-Stokes equations. 


\section{A.1.1 Advective Flux}

The derivative of the advective flux with respect to the conserved state is the third order tensor,

$$
\underline{\mathbf{J}}^{a}(\mathbf{q})=\frac{\partial \overleftrightarrow{\mathbf{f}}^{a}}{\partial \mathbf{q}}
$$

whose components are:

$$
\begin{aligned}
& \underline{\mathbf{J}}_{1}^{a}=\left[\begin{array}{ccccc}
0 & 1 & 0 & 0 & 0 \\
-u^{2}+\frac{1}{2}+(\gamma-1)\|\vec{v}\|^{2} & (3-\gamma) u & -(\gamma-1) v & -(\gamma-1) w & (\gamma-1) \\
-u v & v & u & 0 & 0 \\
-u w & w & 0 & u & 0 \\
u\left(\frac{1}{2}(\gamma-1)\|\vec{v}\|^{2}-H\right) & H-(\gamma-1) u^{2} & -(\gamma-1) u v & -(\gamma-1) u w & \gamma u
\end{array}\right], \\
& \underline{\mathbf{J}}_{2}^{a}=\left[\begin{array}{ccccc}
0 & 0 & 1 & 0 & 0 \\
-u v & v & u & 0 & 0 \\
-v^{2}+\frac{1}{2}+(\gamma-1)\|\vec{v}\|^{2} & -(\gamma-1) u & (3-\gamma) v & -(\gamma-1) w & (\gamma-1) \\
-v w & 0 & w & v & 0 \\
v\left(\frac{1}{2}(\gamma-1)\|\vec{v}\|^{2}-H\right) & -(\gamma-1) u v & H-(\gamma-1) v^{2} & -(\gamma-1) v w & \gamma v
\end{array}\right], \\
& \underline{\mathbf{J}}_{3}^{a}=\left[\begin{array}{ccccc}
0 & 0 & 0 & 1 & 0 \\
-u w & w & 0 & u & 0 \\
-v w & 0 & w & v & 0 \\
-w^{2}+\frac{1}{2}+(\gamma-1)\|\vec{v}\|^{2} & -(\gamma-1) u & -(\gamma-1) v & (3-\gamma) w & (\gamma-1) \\
w\left(\frac{1}{2}(\gamma-1)\|\vec{v}\|^{2}-H\right) & -(\gamma-1) u w & -(\gamma-1) v w & H-(\gamma-1) w^{2} & \gamma w
\end{array}\right],
\end{aligned}
$$

where $H$ is the specific stagnation enthalpy:

$$
H=E+\frac{p}{\rho}
$$

\section{A.1.2 Viscous Flux}

Since the viscous flux, $\stackrel{\leftrightarrow}{\mathbf{f}}^{v}(\mathbf{q}, \vec{\nabla} \mathbf{q})$, depends on both the conserved variables, $\mathbf{q}$, and their gradients, $\vec{\nabla} \mathbf{q}$, it will be useful to define two kinds of gradients, $\underline{\mathbf{J}}$ and $\underline{\underline{\underline{\mathbf{G}}}}$.

Let us first consider the Jacobian with respect to $\vec{\nabla} \mathbf{q}$, when $\mathbf{q}$ is constant. The viscous flux of the compressible Navier-Stokes equations has a linear dependence on $\vec{\nabla} \mathbf{q}$, such that,

$$
\stackrel{\leftrightarrow}{\mathbf{f}}^{v}(\mathbf{q}, \vec{\nabla} \mathbf{q})=\underline{\mathbf{G}}_{i j}(\mathbf{q}) \frac{\partial \mathbf{q}}{\partial x_{j}} \hat{\imath}_{i}
$$


Here, the matrices $\underline{\mathbf{G}}_{i j}$ are components of the fourth order tensor,

$$
\underline{\underline{\underline{\mathbf{G}(\mathbf{q})}}}=\left.\frac{\partial \stackrel{\leftrightarrow}{\mathbf{f}^{v}}}{\partial(\vec{\nabla} \mathbf{q})}\right|_{\mathbf{q}=\text { const }}
$$

The Jacobians for the flux in the x-direction are,

$$
\begin{aligned}
& \underline{\mathbf{G}}_{11}=\frac{\mu}{\rho \operatorname{Re}}\left[\begin{array}{ccccc}
0 & 0 & 0 & 0 & 0 \\
-\frac{4}{3} u & \frac{4}{3} & 0 & 0 & 0 \\
-v & 0 & 1 & 0 & 0 \\
-w & 0 & 0 & 1 & 0 \\
-\left(\frac{1}{3} u^{2}+\|\vec{v}\|^{2}+\frac{\gamma}{\operatorname{Pr}}\left(E-\|\vec{v}\|^{2}\right)\right) & u\left(\frac{4}{3}-\frac{\gamma}{\operatorname{Pr}}\right) & v\left(1-\frac{\gamma}{\operatorname{Pr}}\right) & w\left(1-\frac{\gamma}{\operatorname{Pr}}\right) & \frac{\gamma}{\operatorname{Pr}}
\end{array}\right], \\
& \underline{\mathbf{G}}_{12}=\frac{\mu}{\rho R e}\left[\begin{array}{ccccc}
0 & 0 & 0 & 0 & 0 \\
\frac{2}{3} v & 0 & -\frac{2}{3} & 0 & 0 \\
-u & 1 & 0 & 0 & 0 \\
0 & 0 & 0 & 0 & 0 \\
-\frac{1}{3} u v & v & -\frac{2}{3} u & 0 & 0
\end{array}\right], \underline{G}_{13}=\frac{\mu}{\rho R e}\left[\begin{array}{ccccc}
0 & 0 & 0 & 0 & 0 \\
\frac{2}{3} w & 0 & 0 & -\frac{2}{3} & 0 \\
0 & 0 & 0 & 0 & 0 \\
-u & 1 & 0 & 0 & 0 \\
-\frac{1}{3} u w & w & 0 & -\frac{2}{3} u & 0
\end{array}\right]
\end{aligned}
$$

the Jacobians for the flux in the y-direction are:

$$
\begin{aligned}
& \underline{\mathbf{G}}_{22}=\frac{\mu}{\rho \operatorname{Re}}\left[\begin{array}{ccccc}
0 & 0 & 0 & 0 & 0 \\
-u & 1 & 0 & 0 & 0 \\
-\frac{4}{3} v & 0 & \frac{4}{3} & 0 & 0 \\
-w & 0 & 0 & 1 & 0 \\
-\left(\frac{1}{3} v^{2}+\|\vec{v}\|^{2}+\frac{\gamma}{\operatorname{Pr}}\left(E-\|\vec{v}\|^{2}\right)\right) & u\left(1-\frac{\gamma}{\operatorname{Pr}}\right) & v\left(\frac{4}{3}-\frac{\gamma}{\operatorname{Pr}}\right) & w\left(1-\frac{\gamma}{\operatorname{Pr}}\right) & \frac{\gamma}{\operatorname{Pr}}
\end{array}\right] \text {, } \\
& \underline{\mathbf{G}}_{21}=\frac{\mu}{\rho R e}\left[\begin{array}{ccccc}
0 & 0 & 0 & 0 & 0 \\
-v & 0 & 1 & 0 & 0 \\
\frac{2}{3} u & -\frac{2}{3} & 0 & 0 & 0 \\
0 & 0 & 0 & 0 & 0 \\
-\frac{1}{3} u v & -\frac{2}{3} v & u & 0 & 0
\end{array}\right], \underline{G}_{23}=\frac{\mu}{\rho R e}\left[\begin{array}{ccccc}
0 & 0 & 0 & 0 & 0 \\
0 & 0 & 0 & 0 & 0 \\
\frac{2}{3} w & 0 & 0 & -\frac{2}{3} & 0 \\
-v & 0 & 1 & 0 & 0 \\
-\frac{1}{3} v w & 0 & w & -\frac{2}{3} v & 0
\end{array}\right]
\end{aligned}
$$

and the Jacobians for the flux in the z-direction are,

$$
\underline{\mathbf{G}}_{31}=\frac{\mu}{\rho \operatorname{Re}}\left[\begin{array}{ccccc}
0 & 0 & 0 & 0 & 0 \\
-w & 0 & 0 & 1 & 0 \\
0 & 0 & 0 & 0 & 0 \\
\frac{2}{3} u & -\frac{2}{3} & 0 & 0 & 0 \\
-\frac{1}{3} u w & -\frac{2}{3} w & 0 & u & 0
\end{array}\right], \underline{G}_{32}=\frac{\mu}{\rho \operatorname{Re}}\left[\begin{array}{ccccc}
0 & 0 & 0 & 0 & 0 \\
0 & 0 & 0 & 0 & 0 \\
-w & 0 & 0 & 1 & 0 \\
\frac{2}{3} v & 0 & -\frac{2}{3} & 0 & 0 \\
-\frac{1}{3} v w & 0 & -\frac{2}{3} w & v & 0
\end{array}\right]
$$




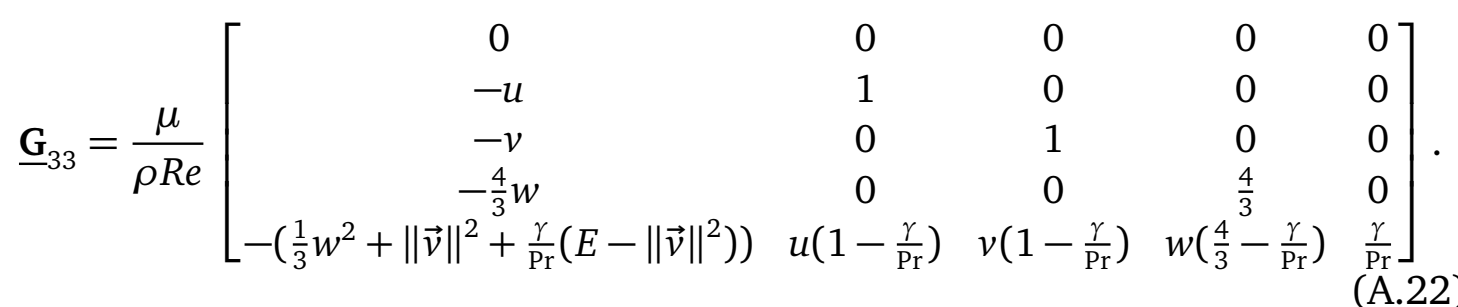

On the other hand, the Jacobian with respect to $\mathbf{q}$ is the third order tensor,

$$
\underline{\underline{\mathbf{J}}}^{v}(\mathbf{q}, \vec{\nabla} \mathbf{q})=\left.\frac{\partial \overleftrightarrow{\mathbf{f}^{v}}}{\partial \mathbf{q}}\right|_{\vec{\nabla} \mathbf{q}=\text { const }},
$$

which is defined in terms of the derivative of Sutherland's law,

$$
\frac{\partial \mu}{\partial \mathbf{q}}=\frac{1+T_{\text {suth }} / T_{\infty}}{T+T_{\text {suth }} / T_{\infty}} \sqrt{T}\left(\frac{3}{2}-\frac{T}{T+T_{\text {suth }} / T_{\infty}}\right) \frac{\partial T}{\partial \mathbf{q}},
$$

where

$$
\frac{\partial T}{\partial \mathbf{q}}=\left[\begin{array}{lllll}
\|\vec{v}\|^{2}-E & -u & -v & -w & 1
\end{array}\right]
$$

The components of $\underline{\mathbf{J}}^{v}$ are written in equations (A.26), (A.27) and (A.28). 


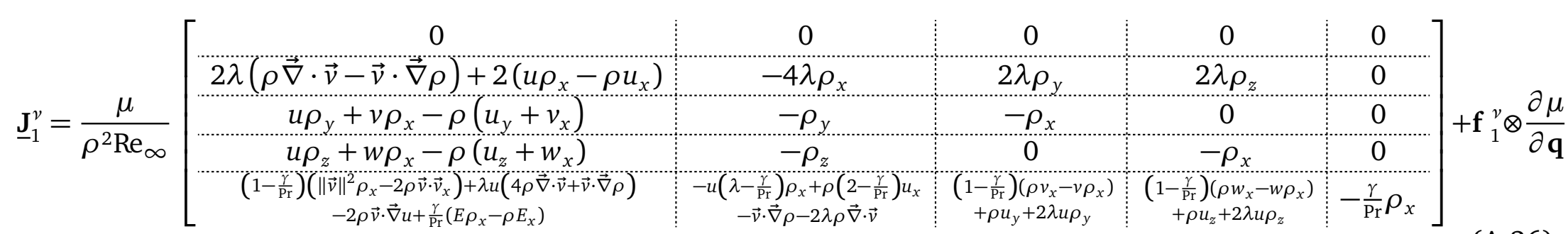

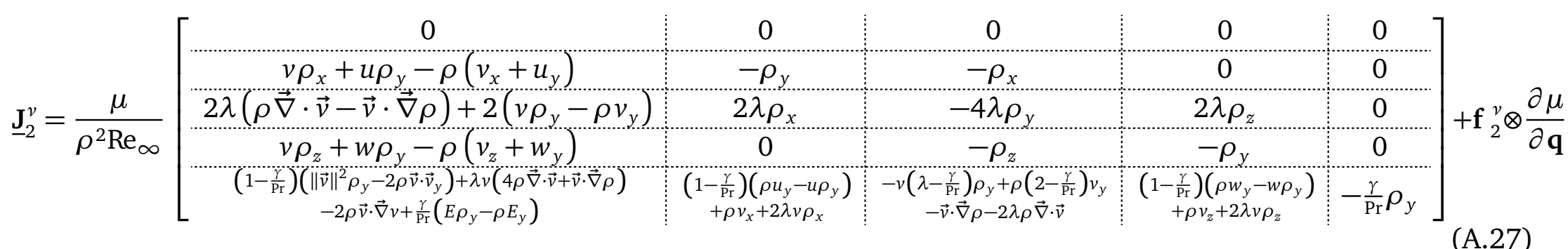

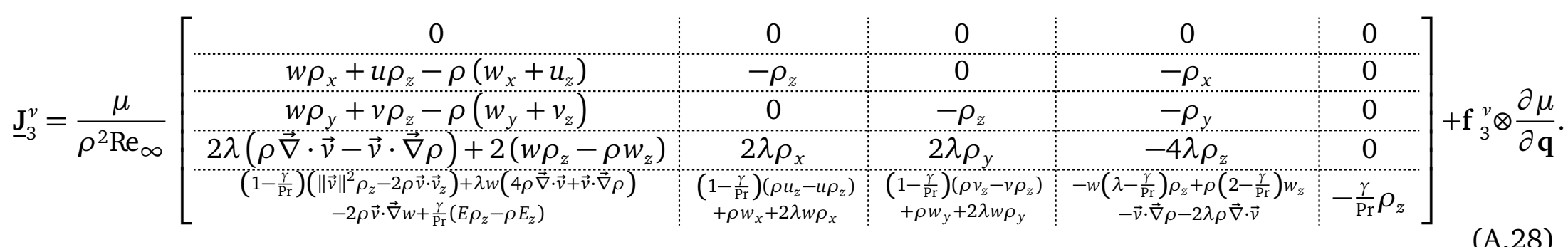




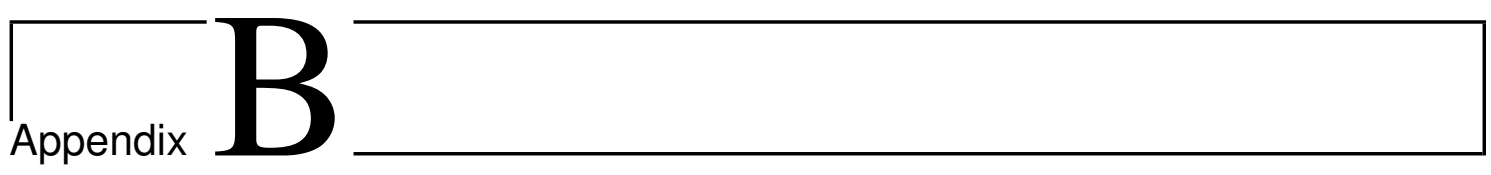

\section{Alternative Forms to Discretize the Viscous Terms}

In Section 2.1.1, we presented the conservative gradients approach to discretize conservation laws that have viscous terms. The conservative gradients approach relies on the fact that viscous fluxes can usually be written as a function of the gradients of the conservative variables.

Besides the conservative gradients approach, the viscous terms may also be discretized using the primitive gradients approach or the whole flux approach.

\section{B.1 Primitive Gradients Approach}

The primitive gradients approach is useful when the viscous flux of the PDE can be written as a function of the gradients of some primitive variables that are not necessarily the conserved quantities. For instance, the viscous flux of the compressible Navier-Stokes equations (see Appendix A) depends solely on the gradients of the velocity and the temperature. As a result, it can be written as

$$
\stackrel{\leftrightarrow}{\mathbf{f}}^{v}(\mathbf{q}, \vec{\nabla} \mathbf{u})
$$

where $\mathbf{u}=[u, v, w, T]^{T}$.

This dependency allows one to define the auxiliary block vector, $\stackrel{\mathrm{g}}{\mathrm{as}}$

$$
\overleftrightarrow{\mathbf{g}}=\vec{\nabla} \mathbf{u}
$$

As a result, the original system of conservation laws is split into two equations,

$$
\left\{\begin{array}{rlr}
\partial_{t} \mathbf{q}+\vec{\nabla} \cdot\left(\overleftrightarrow{\mathbf{f}}^{a}(\mathbf{q})-\overleftrightarrow{\mathbf{f}}^{v}(\mathbf{q}, \overleftrightarrow{\mathbf{g}})\right) & =\mathbf{s} & \text {, in } \Omega, \\
\vec{\nabla} \mathbf{u} & =\overleftrightarrow{\mathbf{g}} \text {, in } \Omega .
\end{array}\right.
$$

The system (B.3) can be solved using the methods detailed in Chapter 2, but there is one additional advantage: The second equation in (B.3) has a smaller size and, therefore, solving it requires less computational power and storage. 
Two main drawbacks of this approach can be identified. First, it only applies to certain conservation laws. Second, since in the derivation of analytical Jacobians for time-integration schemes we want to linearize all the terms with respect a unique variable, usually $\mathbf{q}$, the primitive gradients approach implies doing an extra operation to relate $\vec{\nabla} \mathbf{u}$ with $\vec{\nabla} \mathbf{q}$.

\section{B.2 Whole Flux Approach}

The whole flux approach has been used by Hartmann [91] and Gassner [76]. It is also called the ultra weak formulation by the latter author.

The whole flux approach defines the auxiliary block vector as $\overleftrightarrow{\mathbf{g}}=\overleftrightarrow{\mathbf{f}}^{v}$. Therefore, the split system of equations becomes

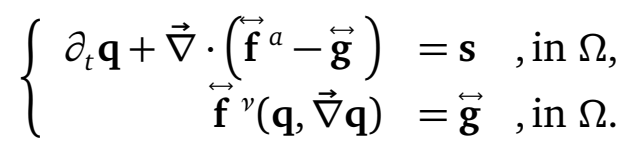

This approach is useful when the viscous flux has a linear dependence on the gradient, as in the Navier-Stokes equations (see Appendix A)

$$
\overleftrightarrow{\mathbf{g}}=\underline{\mathbf{G}}_{i j}(\mathbf{q}) \frac{\partial \mathbf{q}}{\partial x_{j}} \hat{\imath}_{i}
$$

The first equation can be discretized with the strategy presented in Chapter 2. From (2.8), and dropping the superindex $e$ for readability, the first equation of (B.4) becomes

$$
\left\langle\mathbf{q}_{t}^{N}, \phi_{j}\right\rangle_{\Omega}-\left\langle\overleftrightarrow{\mathbf{f}}^{a}, \vec{\nabla} \phi_{j}\right\rangle_{\Omega}+\left\langle\overleftrightarrow{\mathbf{g}}^{N}, \vec{\nabla} \phi_{j}\right\rangle_{\Omega}+\int_{\partial \Omega}\left(\hat{\mathbf{f}}^{a}-\hat{\mathbf{g}}\right) \phi_{j} \mathrm{~d} S=\left\langle\mathbf{s}^{N}, \phi_{j}\right\rangle_{\Omega}
$$

Second equation is multiplied by a vector test function, $\vec{\varphi}$, and integrated by parts twice to obtain

$$
\begin{aligned}
\int_{\Omega} \overleftrightarrow{\mathbf{g}} \cdot \vec{\varphi} d \Omega & =\int_{\Omega} \underline{\mathbf{G}}_{r s}(\mathbf{q}) \frac{\partial \mathbf{q}}{\partial x_{s}} \varphi_{r} d \Omega+\int_{\partial \Omega} \varphi_{r} \underline{\mathbf{G}}_{r s}(\hat{\mathbf{q}}-\mathbf{q}) n_{s} d S \\
& =\int_{\Omega} \mathbf{f}_{r}^{v} \varphi_{r} d \Omega+\int_{\partial \Omega} \varphi_{r} \underline{\mathbf{G}}_{r s}(\hat{\mathbf{q}}-\mathbf{q}) n_{s} d S \\
\langle\overleftrightarrow{\mathbf{g}}, \vec{\varphi}\rangle_{\Omega} & =\left\langle\overleftrightarrow{\mathbf{f}}^{v}, \vec{\varphi}\right\rangle_{\Omega}+\int_{\partial \Omega} \varphi_{r} \underline{\mathbf{G}}_{r s}(\hat{\mathbf{q}}-\mathbf{q}) n_{s} d S,
\end{aligned}
$$

where $\hat{\mathbf{q}}$ is the numerical trace of the solution state vector. Using $\vec{\varphi}=\vec{\nabla} \phi_{j}$ and inserting equation (B.9) in (B.6), we recover the DG discretization: 


$$
\left\langle\mathbf{q}_{t}, \phi_{j}\right\rangle_{\Omega}-\left\langle\stackrel{\mathbf{f}}{\vec{\nabla}} \vec{\nabla} \phi_{j}\right\rangle_{\Omega}+\int_{\partial \Omega} \phi_{j}\left(\mathbf{f}^{a *}-\mathbf{g}^{*}\right) \mathrm{d} S+\int_{\partial \Omega} \frac{\partial \phi}{\partial x_{r}} \mathbf{G}_{r s}(\hat{\mathbf{q}}-\mathbf{q}) n_{s} \mathrm{~d} S=\left\langle\mathbf{s}^{N}, \phi_{j}\right\rangle_{\Omega}
$$

The main advantage is that (B.10) condenses the system (B.4) in just one mathematical expression. This was shown to be advantageous to obtain the analytical Jacobian by Hartmann [91].

The drawback of this approach is the requirement of the viscous flux to be linearly dependent on the gradient of the conservative variables (B.5). 



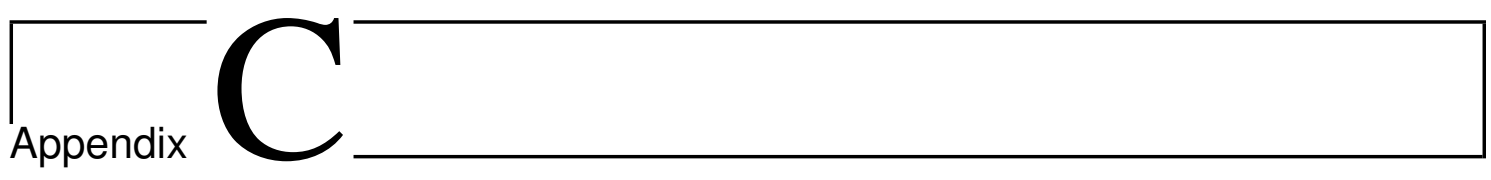

\section{HORSES3D: A High Order (DG) Spectral Element Solver}

HORSES3D is a 3D parallel code that uses the high-order Discontinuous Galerkin Spectral Element Method (DGSEM) and is written in modern object-oriented Fortran $(2008+)$. The code is currently being developed at ETSIAE-UPM (the School of Aeronautics of the Universidad Politécnica de Madrid). HORSES3D is built on the NSLITE3D code by David A. Kopriva, which is based on the book "Implementing Spectral Methods for Partial Differential Equations" [113]. The main developers of HORSES3D are Juan Manzanero, Gonzalo Rubio and Andrés M. Rueda-Ramírez.

HORSES3D and provides following features:

- Hybrid (shared and distributed memory) CPU-based parallelization with OpenMP and MPI.

- A multiphysics environment where the compressible Euler equations of gas dynamics, the compressible Navier-Stokes equations, the incompressible Navier-Stokes equations with artificial compressibility [186], and the CahnHilliad equation for multiphase flow are implemented.

- Support for standard and split form DGSEM.

- Support for arbitrary high-order, $p$-anisotropic discretizations.

- Implicit time-integration schemes such as the Backward Differentiation Formulas (BDFs) and the Rosenbrock type implicit Runge-Kutta methods.

- Multiple linear solvers for the implicit time-integration schemes: a preconditioned matrix-free GMRES solver, a direct LU solver (the implementation of PARDISO that is present in Intel's Math Kernel Library -MKL), a matrix-based GMRES solver (from the PETSc library [1]) and static-condensation methods.

- Numerical Jacobian computations with a coloring algorithm. 
- Analytical Jacobian computations for nonlinear advection and/or diffusion systems of conservation laws.

- Nonlinear multigrid techniques with explicit (Runge-Kutta schemes) and implicit (BDF + iterative methods) smoothers. Two cycling stratefies are implemented: Full Multigrid (FMG) and V-Cycling.

- Support for curvilinear, hexahedral, conforming meshes in SpecMesh/HOHQMesh format $(*$.mesh) and HOPR [96] format $(*$.h5).

- Static and dynamic $p$-adaptation methods for steady and unsteady simulations that use $p$-anisotropic truncation error estimates.

\section{C.1 Strong Scalability}

The code is equipped with distributed memory parallelization using MPI, and shared memory parallelization using OpenMP. In this section, we present strong scalability tests for HORSES3D.

\section{C.1.1 Shared Memory (OpenMP)}

The shared-memory parallelization distributes the most expensive tasks (i.e. the volume and surface integral computations) to different threads using OpenMP directives.

In this section, a strong scalability test is presented for the sphere test case $\left(\operatorname{Re}_{\infty}=200\right.$, Section 9.5.2). Roe is used as the inviscid Riemann Solver and BR1 as the viscous Riemann Solver.

Two common OpenMP schedules were tested: static and guided. The static schedule divides the loops of elements and faces into equal-sized chunks (or as equal as possible) for the different available threads, i.e.

$$
\text { chunk_size }=\text { num_of_elements/num_of_threads, }
$$

and

$$
\text { chunk_size }=\text { num_of_faces/num_of_threads, }
$$

respectively. The guided schedule gives a smaller chunk of loop iterations to each thread. When a thread finishes, it retrieves another chunk to compute. The chunk size starts large and decreases as the loop computation is completed.

The computation time needed for taking 100 time steps (RK3) was measured using a 2-socket Intel CPU with $2 \times 20$ cores at $2.2 \mathrm{GHz}$ and 529 GB RAM. Two discretizations with roughly the same number of DOFs $( \pm 5 \%)$ were used:

(a) A uniform order $\left(N_{1}=N_{2}=N_{3}=5\right)$ discretization (411264 DOFs). 
(b) The $p$-anisotropic discretization that was obtained with the $\tau$-based $p$-adaptation procedure for $\hat{\tau}=10^{-4}$, which provided an improved accuracy (comparable to the $N=N_{\max }=7$ discretization) for a similar number of degrees of freedom (431667 DOFs).

Each of the simulations was run five times and the computation time was averaged. The results are shown in Figure C.1.

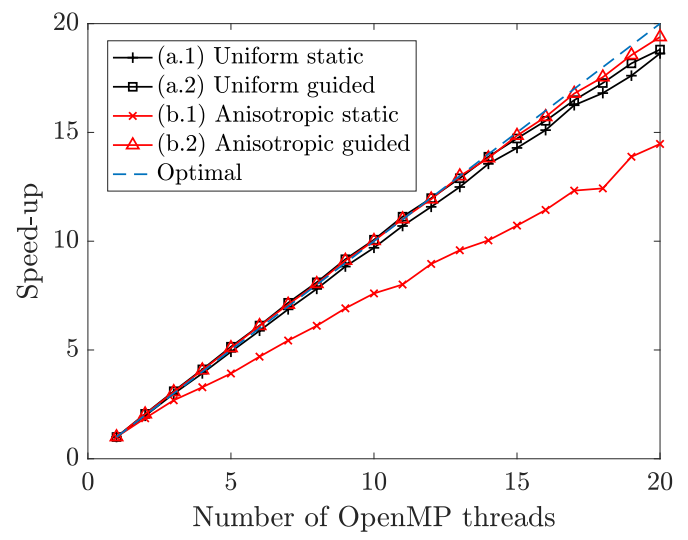

Figure C.1: Strong OpenMP scalability test.

As can be seen in Figure C.1, the code has a near-optimal scalability when using OpenMP + guided schedule. Only the anisotropically $p$-adapted discretization performs suboptimally when the OpenMP schedule is static. However, in OpenMP the guided schedule provides an efficient load balancing.

\section{C.1.2 Distributed Memory (MPI/OpenMP+MPI)}

The benchmark solved herein is the inviscid Taylor-Green Vortex (TGV) problem. Hence, we solve the Euler equations (Appendix A) in a periodic box in the domain $[-\pi, \pi]^{3}$, where we set the initial condition,

$$
\left\{\begin{aligned}
\rho_{0} & =1 \\
(\rho u)_{0} & =\sin (x) \cos (y) \cos (z) \\
(\rho v)_{0} & =-\cos (x) \sin (y) \cos (z) \\
(\rho w)_{0} & =0 \\
p_{0} & =100+\frac{1}{16}(\cos (2 x) \cos (2 z)+2 \cos (y)+2 \cos (2 x)+\cos (2 y) \cos (2 z))
\end{aligned}\right.
$$

We consider a coarse mesh with $32^{3}$, and a fine mesh with $64^{3}$ elements, and we vary the polynomial order from $N=3$ to $N=6$. The solver scalability curves 
are depicted in Figures C.2 and C.3. We consider both pure MPI, and Hybrid (OpenMP+MPI) strategies. The number of total cores ranges from 1 to 700, and the number of OpenMP threads, when enabled, is 48.

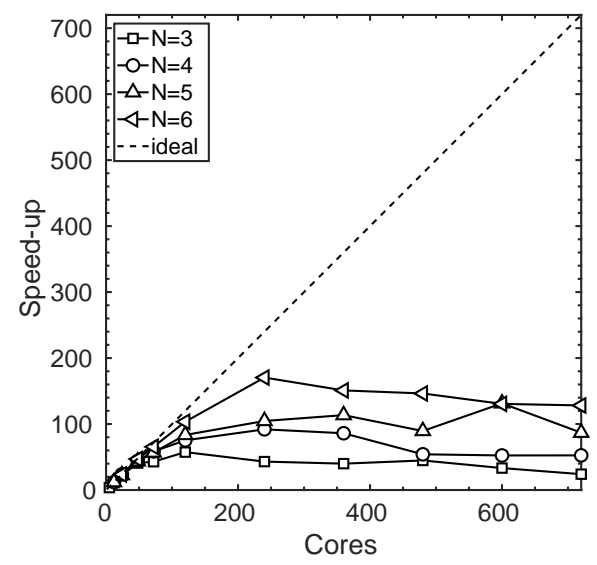

(a) MPI parallelization

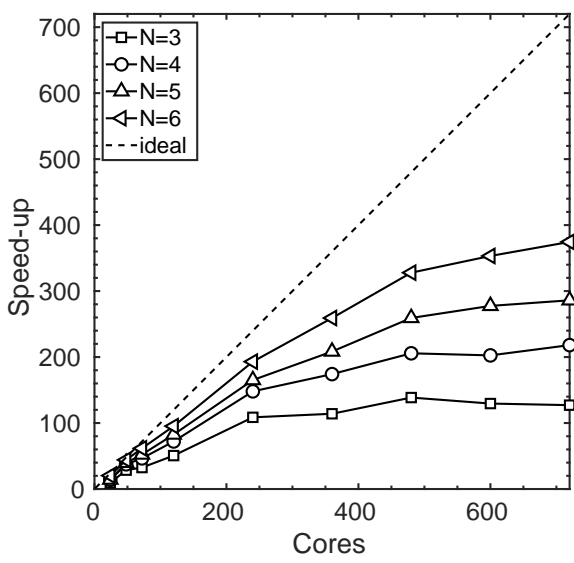

(b) Hybrid MPI+OpenMP parallelization

Figure C.2: Strong scalability solving the TGV problem with a $32^{3}$ mesh.

The $32^{3}$ mesh, using MPI (Figure C.2(a)) does not show an increase in the solver speed-up when increasing the number of cores. Since this mesh is coarse (81 elements per core when using 400 cores, 54 elements per core when using 600), it seems normal that communication time is the bottleneck in this simulation. However, with this configuration it is possible to obtain speed-ups of 100 using 100 cores, depending on the polynomial order. The Hybrid strategy C.2(b), on the other hand, is capable to maintain high speed-ups before the stagnation, approximately on 500 cores. The higher the polynomial order, the higher the speed-up since more calculations inside the elements are performed (i.e. they do not involve communication).

The medium mesh is represented in Figure C.3. Regarding the MPI strategy, Figure C.3(a), we observe an improvement when compared to the coarser mesh in Figure C.2(a). We do not observe stagnation for high polynomial orders, and in the rest, the stagnation occurs after using approximately 400 cores. The Hybrid strategy in Figure C.3(b) does not show stagnation in the range considered. Despite being slighly irregular for lower polynomial orders, a straight line is achieved with $N=6$. 


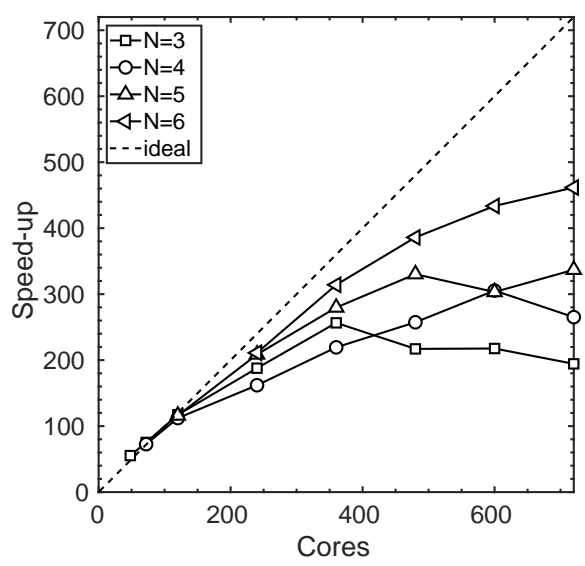

(a) MPI parallelization

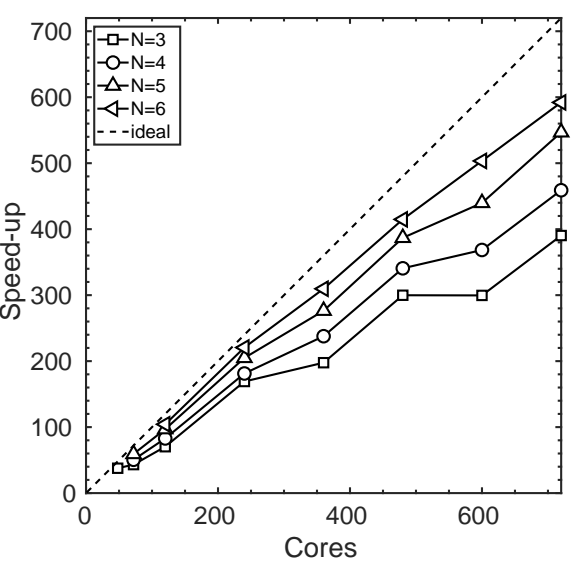

(b) Hybrid MPI+OpenMP parallelization

Figure C.3: Strong scalability solving the TGV problem with a $64^{3}$ mesh. 



\section{Bibliography}

[1] S. Abhyankar, J. Brown, E. M. Constantinescu, D. Ghosh, B. F. Smith, and H. Zhang. PETSc/TS: A Modern Scalable ODE/DAE Solver Library. arXiv preprint arXiv:1806.01437, 2018. Cited on page(s) 51, 72, 91, 203.

[2] M. J. Aftosmis. Upwind method for simulation of viscous flow on adaptively refined meshes. AIAA Journal, 32(2):268-277, 1994. Cited on page(s) 99.

[3] H. B. An, J. Wen, and T. Feng. On finite difference approximation of a matrixvector product in the Jacobian-free Newton Krylov method. Journal of Computational and Applied Mathematics, 236(6):1399-1409, 2011. Cited on page(s) 60.

[4] E. Anderson, Z. Bai, C. Bischof, S. Blackford, J. Demmel, J. Dongarra, J. Du Croz, A. Greenbaum, S. Hammarling, A. McKenney, and D. Sorensen. LAPACK Users' Guide. Society for Industrial and Applied Mathematics, Philadelphia, PA, third edition, 1999. Cited on page(s) 87.

[5] A. F. Antoniadis, D. Drikakis, B. Zhong, G. Barakos, R. Steijl, M. Biava, L. Vigevano, A. Brocklehurst, O. Boelens, M. Dietz, M. Embacher, and W. Khier. Assessment of CFD methods against experimental flow measurements for helicopter flows. Aerospace Science and Technology, 19(1):86-100, 2012. Cited on page(s) 1, 5.

[6] D. N. Arnold, F. Brezzi, B. Cockburn, and D. Marini. Unified analysis of discontinuous Galerkin methods for elliptic problems. SIAM J. Numer. Anal., 39(5):1749-1779, 2002. Cited on page(s) 22, 24, 25, 68, 78.

[7] W. E. Arnoldi. The principle of minimized iterations in the solution of the matrix eigenvalue problem. Quarterly of Applied Mathematics, 9(1):17-29, 1951. Cited on page(s) 49.

[8] A. Arnone. Integration of Navier-Stokes equations using dual time stepping and a multigrid method. AIAA Journal, 33(6):985-990, 1995. Cited on page(s) 172. 
[9] I. Babuska, J. E. Flaherty, W. D. Henshaw, J. E. Hopcroft, J. E. Oliger, and T. Tezduyar. Modeling, mesh generation, and adaptive numerical methods for partial differential equations, volume 75. Springer Science \& Business Media, 2012. Cited on page(s) 3.

[10] I. Babuška and A. Miller. The post-processing approach in the finite element method-part 1: Calculation of displacements, stresses and other higher derivatives of the displacements. International Journal for numerical methods in engineering, 20(6):1085-1109, 1984. Cited on page(s) 99.

[11] I. Babuška and A. Miller. The post-processing approach in the finite element method-Part 2: The calculation of stress intensity factors. International Journal for numerical methods in Engineering, 20(6):1111-1129, 1984. Cited on page(s) 99.

[12] F. Bassi, L. Botti, A. Colombo, A. Ghidoni, and F. Massa. Linearly implicit Rosenbrock-type Runge-Kutta schemes applied to the Discontinuous Galerkin solution of compressible and incompressible unsteady flows. Computers \& Fluids, 118:305-320, 2015. Cited on page(s) 6.

[13] F. Bassi, D. Ghidoni, S. Rebay, and P. Tesini. High-order accurate p-multigrid discontinuous Galerkin solution of the Euler Equations. International Journal for Numerical Methods in Fluids, 60(24 September 2008):847-865, 2009. Cited on page(s) 7, 42, 44, 45.

[14] F. Bassi and F. Rebay. A high-order accurate discontinuous finite element method for the numerical solution of the compressible Navier-Stokes equations. Journal of Computational Physics, 131:267-279, 1997. Cited on page(s) 4, 24, 181.

[15] F. Bassi, S. Rebay, G. Mariotti, S. Pedinotti, and M. Savini. A high-order accurate discontinuous finite element method for inviscid and viscous turbomachinery flows. In Proceedings of the 2nd European Conference on Turbomachinery Fluid Dynamics and Thermodynamics, pages 99-109. Technologisch Instituut, Antwerpen, Belgium, 1997. Cited on page(s) 24.

[16] A. D. Beck, T. Bolemann, D. Flad, H. Frank, G. J. Gassner, F. Hindenlang, and C.-D. Munz. High-order discontinuous Galerkin spectral element methods for transitional and turbulent flow simulations. International Journal for Numerical Methods in Fluids, 76:522-548, 2014. Cited on page(s) 5.

[17] A. D. Beck, D. G. Flad, C. Tonhäuser, G. Gassner, and C. D. Munz. On the Influence of Polynomial De-aliasing on Subgrid Scale Models. Flow, Turbulence and Combustion, 97(2):475-511, 2016. Cited on page(s) 5. 
[18] R. Becker and R. Rannacher. Weighted A Posteriori Error Control in FE Methods. ENUMATH-97, Heidelberg, pages $621-637,1996$. Cited on page(s) 99.

[19] M. J. Berger. Adaptive finite difference methods in fluid dynamics. In In Von Karman Inst. for Fluid Dynamics, Computational Fluid Dynamics 50 p (SEE N88-15951 08-34), pages 08-34, 1987. Cited on page(s) 100, 101.

[20] A. Bhatia, S. Roy, and R. Gosse. 2-D Hypersonic Non-equilibrium Flow Simulation using r-p Adaptive Time-Implicit Discontinuous Galerkin Method. In 51st Aerospace Sciences Meeting, 2013. Cited on page(s) 129.

[21] P. Birken. Numerical methods for the unsteady compressible Navier-Stokes equations. 2013. Cited on page(s) 31, 41, 48, 51.

[22] P. Birken, G. Gassner, M. Haas, and C.-d. Munz. Efficient Time Integration for Discontinous Galaerkin Method for the Unsteady 3D Navier-Stokes Equations. In European Congress on Computational Methods and Applied Sciences and Engineering (ECCOMAS 2012), pages 1-20, 2012. Cited on page(s) 36.

[23] K. Black. A conservative spectral element method for the approximation of compressible fluid flow. Kybernetika, 35(1):133-146, 1999. Cited on page(s) 5, 25.

[24] L. S. Blackford, A. Petitet, R. Pozo, K. Remington, R. C. Whaley, J. Demmel, J. Dongarra, I. Duff, S. Hammarling, G. Henry, and Others. An updated set of basic linear algebra subprograms (BLAS). ACM Transactions on Mathematical Software, 28(2):135-151, 2002. Cited on page(s) 87.

[25] S. Blaise and A. St-Cyr. A Dynamic hp -Adaptive Discontinuous Galerkin Method for Shallow-Water Flows on the Sphere with Application to a Global Tsunami Simulation . Monthly Weather Review, 140(3):978-996, 2012. Cited on page(s) 173.

[26] M. Bohm, A. R. Winters, G. J. Gassner, D. Derigs, F. Hindenlang, and J. Saur. An entropy stable nodal discontinuous Galerkin method for the resistive MHD equations. Part I: Theory and numerical verification. Journal of Computational Physics, 1:1-35, 2018. Cited on page(s) 5, 27, 74.

[27] L. Botti. Influence of reference-to-physical frame mappings on approximation properties of discontinuous piecewise polynomial spaces. Journal of Scientific Computing, 52(3):675-703, 2012. Cited on page(s) 138.

[28] L. Botti, A. Colombo, and F. Bassi. h-multigrid agglomeration based solution strategies for discontinuous Galerkin discretizations of incompressible flow problems. Journal of Computational Physics, 347:382-415, 2017. Cited on page(s) 7, 42, 44. 
[29] M. Braack. Finite Elements With Local Projection Stabilization for Incompressible Flow Problems. Journal of Computational Mathematics, 27(2):116147, 2009. Cited on page(s) 3.

[30] M. Braack and E. Burman. Local Projection Stabilization for the Oseen Problem and its Interpretation as a Variational Multiscale Method. SIAM Journal on Numerical Analysis, 43(6):2544-2566, 2012. Cited on page(s) 3.

[31] F. Bramkamp, P. Lamby, and S. Müller. An adaptive multiscale finite volume solver for unsteady and steady state flow computations. Journal of Computational Physics, 197(2):460-490, 2004. Cited on page(s) 3.

[32] A. Brandt. Multi-level adaptive solutions to boundary-value problems. Mathematics of Computation, 31(138):333-390, 1977. Cited on page(s) 41, 45, 46.

[33] A. Brandt and O. E. Livne. Multigrid Techniques: 1984 Guide with Applications to Fluid Dynamics, Revised Edition. SIAM, 2011. Cited on page(s) 3, 42, 46, 52, 101, 144.

[34] W. L. Briggs, S. F. McCormick, and Others. A multigrid tutorial, volume 72. Siam, 2000. Cited on page(s) 3.

[35] O. M. F. Browne, G. Rubio, E. Ferrer, and E. Valero. Sensitivity analysis to unsteady perturbations of complex flows: A discrete approach. International Journal for Numerical Methods in Fluids, 76(12):1088-1110, 2014. Cited on page(s) 5, 57.

[36] G. L. Bryan, M. L. Norman, B. W. O. Shea, T. Abel, J. H. Wise, M. J. Turk, D. R. Reynolds, D. C. Collins, P. Wang, S. W. Skillman, B. Smith, R. P. Harkness, J. Bordner, J.-h. Kim, M. Kuhlen, H. Xu, and N. Goldbaum. ENZO : An Adaptive Mesh Refinement Code for Astrophysics. The Astrophysical Journal Supplement Series, 211(2):1-52, 2014. Cited on page(s) 1, 5.

[37] N. K. Burgess. An Adaptive Discontinuous Galerkin Solver for Aerodynamic Flows. PhD thesis, University of Wyoming, 2011. Cited on page(s) 98, 129, 153.

[38] J. S. Cagnone and S. K. Nadarajah. A stable interface element scheme for the p-adaptive lifting collocation penalty formulation. Journal of Computational Physics, 231(4):1615-1634, 2012. Cited on page(s) 173.

[39] X.-C. Cai and M. Sarkis. A Restricted Additive Schwarz Preconditioner for Feneral Sparse Linear Systems. SIAM Journal on Scientific Computing, 21(2):792-797, 1999. Cited on page(s) 3. 
[40] L. Cambier. M echanics I ndustry The Onera elsA CFD software : input from research and feedback from industry. Mechanics \& Industry, 14(2013):159174, 2019. Cited on page(s) 1, 5.

[41] R. Campregher, J. Militzer, R. Campregher, S. S. Mansur, and A. da Silveira Neto. Computations of the flow past a still sphere at moderate reynolds numbers using an immersed boundary method. Journal of the Brazilian Society of Mechanical Sciences and Engineering, 31(4):344-352, 2009. Cited on page(s) 158.

[42] C. Canuto, M. Y. Hussaini, A. Quarteroni, A. Thomas Jr, and Others. Spectral methods in fluid dynamics. Springer Science \& Business Media, 2012. Cited on page(s) 4, 102, 105.

[43] J. Carrero, B. Cockburn, and D. Schötzau. Hybridized globally divergencefree LDG methods. Part I: The Stokes problem. Mathematics of Computation, 75(254):533-564, 2005. Cited on page(s) 75, 76, 78.

[44] Casoni, E. and Huerta, A. Shock capturing for discontinuous galerkin methods, 2011. Cited on page(s) 100.

[45] S. C. Chapra, R. P. Canale, and Others. Numerical methods for engineers. Boston: McGraw-Hill Higher Education, 2010. Cited on page(s) 32.

[46] A. Choudhary and C. J. Roy. Structured Mesh r-Refinement using Truncation Error Equidistribution for 1D and 2D Euler Problems. In 21st AIAA Computational Fluid Dynamics Conference, page 2444. American Institute of Aeronautics and Astronautics, Reston, Virginia, 2013. Cited on page(s) 100 .

[47] B. Cockburn, J. Gopalakrishnan, and R. Lazarov. Unified Hybridization of Discontinuous Galerkin, Mixed, and Continuous Galerkin Methods for Second Order Elliptic Problems. Society for Industrial and Applied Mathematics, 47(2):1319-1365, 2009. Cited on page(s) 75, 76, 78.

[48] B. Cockburn, G. E. Karniadakis, and C.-W. Shu. The Development of Discontinuous Galerkin Methods. Discontinuous Galerkin Methods, 11(0):3-50, 2000. Cited on page(s) 4.

[49] B. Cockburn and C.-W. Shu. The Local Discontinuous Galerkin Method for Time-Dependent Convection-Diffusion Systems. SIAM Journal on Numerical Analysis, 35(6):2440-2463, 1998. Cited on page(s) 4.

[50] T. F. Coleman and J. J. Moré. Estimation of Sparse Jacobian Matrices and Graph Coloring Problems. SIAM Journal on Numerical Analysis, 20(1):187209, 1983. Cited on page(s) 58. 
[51] A. W. Craig and O. C. Zienkiewicz. A multigrid algorithm using a hierarchical finite element basis. In Multigrid Methods for Integral and Differential Equations, pages 301-312. Clarendon Press, Oxford, 1985. Cited on page(s) 41.

[52] A. De Coninck, B. De Baets, D. Kourounis, F. Verbosio, O. Schenk, S. Maenhout, and J. Fostier. Needles: Toward Large-Scale Genomic Prediction with Marker-by-Environment Interaction. Genetics, 203(1):543-555, 2016. Cited on page(s) 47.

[53] B. de Veubeke. Displacement and equilibrium models in the finite element method. Stress analysis, pages chapter--9, 1965. Cited on page(s) 75, 76, 77.

[54] S. Deng. Numerical simulation of optical coupling and light propagation in coupled optical resonators with size disorder. Applied Numerical Mathematics, 57(5-7 SPEC. ISS.):475-485, 2007. Cited on page(s) 5.

[55] J. E. Dennis, Jr. and J. J. Moré. Quasi-Newton Methods, Motivation and Theory. SIAM Review, 19(1):46-89, 1977. Cited on page(s) 41.

[56] J. Douglas and T. Dupont. Interior Penalty Procedures for Elliptic and Parabolic Galerkin Methods. Computing Methods in Applied Sciences, pages 207-216, 2008. Cited on page(s) 24, 25.

[57] R. Eymard, T. Gallouët, and R. Herbin. Finite volume methods. Handbook of numerical analysis, 7:713-1018, 2000. Cited on page(s) 3.

[58] A. Facius. Iterative Solution of Linear Systems with Improved Arithmetic and Result Verification. PhD thesis, Universität Karlsruhe, 2000. Cited on page(s) 49.

[59] E. A. Fadlun, R. Verzicco, P. Orlandi, and J. Mohd-Yusof. Combined Immersed-Boundary Finite-Difference Methods for Three-Dimensional Complex Flow Simulations. Journal of Computational Physics, 161(1):3560, 2000. Cited on page(s) xxiii, 158.

[60] R. D. Falgout and U. M. Yang. hypre: A Library of High Performance Preconditioners. In P. M. A. Sloot, A. G. Hoekstra, C. J. K. Tan, and J. J. Dongarra, editors, Computational Science - ICCS 2002, pages 632-641, Berlin, Heidelberg, 2002. Springer Berlin Heidelberg. Cited on page(s) 51, 72.

[61] P. Fernandez, N. C. Nguyen, and J. Peraire. The hybridized Discontinuous Galerkin method for Implicit Large-Eddy Simulation of transitional turbulent flows. Journal of Computational Physics, 336(May):308-329, 2017. Cited on page(s) 78 . 
[62] E. Ferrer. A high order Discontinuous Galerkin - Fourier incompressible 3D Navier-Stokes solver with rotating sliding meshes for simulating cross-flow turbines by. PhD thesis, Oxford University, 2012. Cited on page(s) 4.

[63] E. Ferrer. An interior penalty stabilised incompressible discontinuous Galerkin-Fourier solver for implicit large eddy simulations. Journal of Computational Physics, 348:754-775, 2017. Cited on page(s) 4.

[64] E. Ferrer and R. H. Willden. A high order Discontinuous Galerkin - Fourier incompressible 3D Navier-Stokes solver with rotating sliding meshes. Journal of Computational Physics, 231(21):7037-7056, 2012. Cited on page(s) 4, 7, 97.

[65] E. Ferrer and R. H. J. Willden. A high order Discontinuous Galerkin Finite Element solver for the incompressible Navier-Stokes Equations. Computers I\& Fluids, 46(1):224-230, 2011. Cited on page(s) 25.

[66] J. H. Ferziger and M. Peric. Computational methods for fluid dynamics. Springer Science \& Business Media, 2012. Cited on page(s) 2.

[67] K. J. Fidkowski. Output error estimation strategies for discontinuous Galerkin discretizations of unsteady convection-dominated flow. International Journal for Numerical Methods in Engineering, 88:1297-1322, 2011. Cited on page(s) 173.

[68] K. J. Fidkowski, T. A. Oliver, J. Lu, and D. L. Darmofal. p-Multigrid solution of high-order discontinuous Galerkin discretizations of the compressible Navier-Stokes equations. Journal of Computational Physics, 207(1):92-113, 2005. Cited on page(s) 7, 42, 44, 45.

[69] T. C. Fisher, M. H. Carpenter, J. Nordström, N. K. Yamaleev, and C. Swanson. Discretely conservative finite-difference formulations for nonlinear conservation laws in split form: Theory and boundary conditions. Journal of Computational Physics, 234(1):353-375, 2013. Cited on page(s) 74.

[70] B. Fornberg. Steady viscous flow past a sphere at high Reynolds numbers. J. Fluid Mech., 190:471-489, 1988. Cited on page(s) 158.

[71] F. Fraysse, C. Redondo, G. Rubio, and E. Valero. Upwind methods for the Baer-Nunziato equations and higher-order reconstruction using artificial viscosity. Journal of Computational Physics, 326:805-827, 2016. Cited on page(s) 5 .

[72] F. Fraysse, G. Rubio, J. De Vicente, and E. Valero. Quasi-a priori mesh adaptation and extrapolation to higher order using $\tau$-estimation. Aerospace Science and Technology, 38:76-87, 2014. Cited on page(s) 100, 101. 
[73] F. Fraysse, E. Valero, and J. Ponsín. Comparison of Mesh Adaptation Using the Adjoint Methodology and Truncation Error Estimates. AIAA Journal, 50(9):1920-1932, 2012. Cited on page(s) 99, 100, 101, 149.

[74] F. Fraysse, E. Valero, and G. Rubio. Quasi-a priori truncation error estimation and higher order extrapolation for non-linear partial differential equations. Journal of Computational Physics, 253:389-404, 2013. Cited on page(s) 100, 101.

[75] G. Gassner and D. A. Kopriva. A Comparison of the Dispersion and Dissipation Errors of Gauss and Gauss-Lobatto Discontinuous Galerkin Spectral Element Methods. SIAM Journal on Scientific Computing, 33(5):2560-2579, 2011. Cited on page(s) 74.

[76] G. J. Gassner. Discontinuous Galerkin methods for the unsteady compressible Navier-Stokes equations. PhD thesis, University of Stuttgart, 2009. Cited on page(s) 104, 200.

[77] G. J. Gassner. A Skew-Symmetric Discontinuous Galerkin Spectral Element Discretization and Its Relation to SBP-SAT Finite Difference Methods. SIAM Journal on Scientific Computing, 35(3):A1233-A1253, 2013. Cited on page(s) 5, 74.

[78] G. J. Gassner, F. Hindenlang, and C.-D. Munz. A Runge-Kutta Based Discontinuous Galerkin Method With Time Accurate Local Time Stepping. In Adaptive High-Order Methods in Computational Fluid Dynamics, pages 95118. World Scientific, 2011. Cited on page(s) 35.

[79] G. J. Gassner, A. R. Winters, F. J. Hindenlang, and D. A. Kopriva. The BR1 Scheme is Stable for the Compressible Navier - Stokes Equations, volume 77. Springer US, 2018. Cited on page(s) 25.

[80] G. J. Gassner, A. R. Winters, and D. A. Kopriva. Split form nodal discontinuous Galerkin schemes with summation-by-parts property for the compressible Euler equations. Journal of Computational Physics, 327:39-66, 2016. Cited on page(s) 5, 74.

[81] P. Gatto, R. E. Christiansen, and J. S. Hesthaven. A preconditioner based on a low-rank approximation with applications to topology optimization. Journal of Scientific Computing, 2015. Cited on page(s) 93.

[82] C. W. Gear and Y. Saad. Iterative solution of linear equations in ODE codes. SIAM journal on scientific and statistical computing, 4(4):583-601, 1983. Cited on page(s) 60.

[83] E. Georgoulis. Discontinuous Galerkin methods on Shape-Regular and Anisotropic Meshes. Philosophy, 2003. Cited on page(s) 105, 119. 
[84] M. Giles, N. Pierce, M. Giles, and N. Pierce. Adjoint equations in CFD Duality, boundary conditions and solution behaviour. In 13th Computational Fluid Dynamics Conference, page 1850, 2013. Cited on page(s) 99.

[85] A. Griewank. On Automatic Differentiation. Mathematical Programming: recent developments and applications, 6(6):83-107, 1988. Cited on page(s) 72 .

[86] L. Grigori, E. G. Boman, S. Donfack, and T. A. Davis. Hypergraph-based unsymmetric nested dissection ordering for sparse LU factorization. SIAM Journal on Scientific Computing, 32(6):3426-3446, 2010. Cited on page(s) 47.

[87] R. J. Guyan. Reduction of stiffness and mass matrices. AIAA journal, 3(2):380, 1965. Cited on page(s) 75, 76.

[88] W. Habashi, J. Dompierre, Y. Bourgault, D. Ait-Ali-Yahia, M. Fortin, and M.G. Vallet. Anisotropic mesh adaptation: Towards user-independent, meshindependent and solver-independent CFD. Part I: General principles. International Journal for Numerical Methods in Fluids, 32(6):725-744, 2000. Cited on page(s) 3.

[89] E. Haber and U. M. Ascher. Fast Finite Volume Simulation of 3D Electromagnetic Problems with Highly Discontinuous Coefficients. SIAM Journal on Scientific Computing, 22(6):1943-1961, 2001. Cited on page(s) 3.

[90] A. Harten and J. M. Hyman. Self adjusting grid methods for one-dimensional hyperbolic conservation laws. Journal of Computational Physics, 50(2):235269, 1983. Cited on page(s) 24.

[91] R. Hartmann. Discontinuous Galerkin methods for compressible flows: higher order accuracy, error estimation and adaptivity. VKI LS 2006-01: CFD-Higher Order Discretization Methods, pages 1-50, 2005. Cited on page(s) 65, 200, 201.

[92] R. Hartmann. Error estimation and adjoint-based adaptation in aerodynamics. European Conference on Computational Fluid Dynamics, pages 1-14, 2006. Cited on page(s) 99.

[93] R. Hartmann and P. Houston. Adaptive Discontinuous Galerkin Finite Element Methods for the Compressible Euler Equations. Journal of Computational Physics, 183(2):508-532, 2002. Cited on page(s) 99, 100.

[94] L. Haupt, J. Stiller, and W. E. Nagel. A fast spectral element solver combining static condensation and multigrid techniques. Journal of Computational Physics, 255:384-395, 2013. Cited on page(s) 45. 
[95] J. S. Hesthaven and T. Warburton. Nodal discontinuous Galerkin methods: algorithms, analysis, and applications. Springer Science \& Business Media, 2007. Cited on page(s) 4, 23, 34, 102, 104, 105.

[96] F. Hindenlang, T. Bolemann, and C.-D. Munz. Mesh Curving Techniques for High Order Discontinuous Galerkin Simulations. pages 133-152. Springer, 2015. Cited on page(s) 104, 157, 204.

[97] F. Hindenlang, G. J. Gassner, C. Altmann, A. Beck, M. Staudenmaier, and C. D. Munz. Explicit discontinuous Galerkin methods for unsteady problems. Computers and Fluids, 61:86-93, 2012. Cited on page(s) 4, 32, 150.

[98] C. Hirsch. Numerical computation of internal and external flows: The fundamentals of computational fluid dynamics. Elsevier, 2007. Cited on page(s) 31 .

[99] K. A. Hoffmann and S. T. Chiang. Computational fluid dynamics volume I. 2000. Cited on page(s) 2.

[100] K. A. Hoffmann and S. T. Chiang. Computational fluid dynamics volume II. Engineering Education System, fourth edi edition, 2001. Cited on page(s) $2,3$.

[101] M. Hortmann, M. Perić, and G. Scheuerer. Finite volume multigrid prediction of laminar natural convection: Bench-mark solutions. International Journal for Numerical Methods in Fluids, 11(2):189-207, 1990. Cited on page(s) 3, 7, 41.

[102] T. J. Hughes. Recent progress in the development and understanding of SUPG methods with special reference to the compressible Euler and NavierStokes equations. International Journal for Numerical Methods in Fluids, 7(11):1261-1275, 1987. Cited on page(s) 3.

[103] C. Johnson and R. Rannacher. On error control in CFD. In Numerical methods for the Navier-Stokes equations, pages 133-144. Springer, 1994. Cited on page(s) 99.

[104] G. Karniadakis and S. Sherwin. Spectral/hp element methods for computational fluid dynamics. Oxford University Press, 2013. Cited on page(s) 4, 34, 75, 76, 77.

[105] S. S. Khalafvand, E. Y. Ng, and L. Zhong. CFD simulation of flow through heart: A perspective review. Computer Methods in Biomechanics and Biomedical Engineering, 14(1):113-132, 2011. Cited on page(s) 1. 
[106] R. M. Kirby and G. E. Karniadakis. Selecting the Numerical Flux in Discontinuous Galerkin Methods for Diffusion Problems. Journal of Scientific Computing, 22(1-3):385-411, 2005. Cited on page(s) .

[107] C. Klaij and C. Vuik. SIMPLE-type preconditioners for cell-centered, colocated finite volume discretization of incompressible Reynolds-averaged Navier-Stokes equations. International Journal for Numerical Methods in Fluids, 17:830-849, 2013. Cited on page(s) 3.

[108] D. A. Knoll and D. E. Keyes. Jacobian-free Newton-Krylov methods: A survey of approaches and applications. Journal of Computational Physics, 193(2):357-397, 2004. Cited on page(s) 60, 61.

[109] M. Kompenhans. Adaptation Strategies for Discontinuous Galerkin Spectral Element Methods by Means of Truncation Error Estimations. PhD thesis, Universidad Politécnica de Madrid, 2016. Cited on page(s) 116.

[110] M. Kompenhans, G. Rubio, E. Ferrer, and E. Valero. Adaptation strategies for high order discontinuous Galerkin methods based on Tau-estimation. Journal of Computational Physics, 306:216-236, 2016. Cited on page(s) 5, 7, 100, 101, 105, 106, 107, 109, 110, 114, 115, 116, 117, 127, 128, 130, 132, 134, 141, 146, 149, 168, 169.

[111] M. Kompenhans, G. Rubio, E. Ferrer, and E. Valero. Comparisons of padaptation strategies based on truncation- and discretisation-errors for high order discontinuous Galerkin methods. Computers and Fluids, 139:36-46, 2016. Cited on page(s) $98,99,100,101,106,107,110,114,115,168$, 169.

[112] D. A. Kopriva. Metric identities and the discontinuous spectral element method on curvilinear meshes. Journal of Scientific Computing, 26(3):301327, 2006. Cited on page(s) 28, 128, 137.

[113] D. A. Kopriva. Implementing spectral methods for partial differential equations: Algorithms for scientists and engineers. Springer Science \& Business Media, 2009. Cited on page(s) 5, 9, 25, 26, 27, 28, 203.

[114] D. A. Kopriva, F. Hindenlang, T. Boleman, and G. J. Gassner. FreeStream Preservation for Curved Geometrically Non-Conforming Discontinuous Galerkin Spectral Elements. arXiv preprint arXiv:1809.05206, 2018. Cited on page(s) 28 .

[115] D. A. Kopriva, J. Nordström, and G. J. Gassner. Error Boundedness of Discontinuous Galerkin Spectral Element Approximations of Hyperbolic Problems. Journal of Scientific Computing, 72(1):314-330, 2017. Cited on page(s) 104. 
[116] D. A. Kopriva, S. L. Woodruff, and M. Y. Hussaini. Computation of electromagnetic scattering with a non-conforming discontinuous spectral element method. International Journal for Numerical Methods in Engineering, 53(1):105-122, 2002. Cited on page(s) 4, 5, 7, 110, 111.

[117] D. Kourounis, A. Fuchs, and O. Schenk. Towards the Next Generation of Multiperiod Optimal Power Flow Solvers. IEEE Transactions on Power Systems, PP(99):1-10, 2018. Cited on page(s) 47.

[118] A. G. Kravchenko and P. Moin. On the effect of numerical errors in large Eddy simulations of turbulent flows. Journal of Computational Physics, 131(2):310-322, 1997. Cited on page(s) 74.

[119] L. Krivodonova and J. E. Flaherty. Error estimation for discontinuous $\{\mathrm{G}\}$ alerkin solutions of multidimensional hyperbolic problems. Advances in Computational Mathematics, 19:57-71, 2003. Cited on page(s) 99.

[120] L. Krivodonova, J. Xin, J.-F. Remacle, N. Chevaugeon, and J. E. Flaherty. Shock detection and limiting with discontinuous Galerkin methods for hyperbolic conservation laws. Applied Numerical Mathematics, 48(3-4):323338, 2004. Cited on page(s) 99.

[121] G. Kuru and M. De la Llave Plata. An adaptive variational multiscale discontinuous Galerkin method for large eddy simulation. 54th AIAA Aerospace Sciences Meeting, page p. 0584, 2016. Cited on page(s) 100.

[122] R. B. Lehoucq, D. C. Sorensen, and C. Yang. ARPACK users' guide: solution of large-scale eigenvalue problems with implicitly restarted Arnoldi methods, volume 6. Siam, 1998. Cited on page(s) 90.

[123] H.-J. Leister and M. Perié. Numerical simulation of a 3D Czochralski-melt flow by a finite volume multigrid-algorithm. Journal of Crystal Growth, 123:567-574, 1992. Cited on page(s) 7, 41.

[124] R. J. LeVeque. Finite volume methods for hyperbolic problems, volume 31. Cambridge university press, 2002. Cited on page(s) 23.

[125] R. J. LeVeque. Finite difference methods for ordinary and partial differential equations: steady-state and time-dependent problems, volume 98. Siam, 2007. Cited on page(s) 31, 32 .

[126] W. Li, J. Pan, and Y.-x. Ren. The discontinuous Galerkin spectral element methods for compressible flows on two-dimensional mixed grids. Journal of Computational Physics, 364(February):314-346, 2018. Cited on page(s) 165. 
[127] X. S. Li. An Overview of SuperLU: Algorithms, Implementation, and User Interface. ACM Transactions on Mathematical Software (TOMS), 31(3):302325, 2005. Cited on page(s) 47.

[128] X. S. Li, J. W. Demmel, J. R. Gilbert, iL. Grigori, M. Shao, and I. Yamazaki. SuperLU Users' Guide. Technical Report LBNL-44289, Lawrence Berkeley National Laboratory, 1999. Cited on page(s) 47.

[129] T. Liszka, W. Tworzydlo, J. Bass, S. Sharma, T. Westermann, and B. Yavari. ProPHLEX-An hp-adaptive finite element kernel for solving coupled systems of partial differential equations in computational mechanics. Computer Methods in Applied Mechanics and Engineering, 150(1-4):251-271, 1997. Cited on page(s) 153.

[130] F. Lörcher, G. Gassner, and C. D. Munz. An explicit discontinuous Galerkin scheme with local time-stepping for general unsteady diffusion equations. Journal of Computational Physics, 227(11):5649-5670, 2008. Cited on page(s) 35 .

[131] H. Luo, J. D. Baum, and R. Löhner. A p-multigrid discontinuous Galerkin method for the Euler equations on unstructured grids. Journal of Computational Physics, 211(2):767-783, 2006. Cited on page(s) 7, 42, 44.

[132] H. Luo, J. D. Baum, and R. Löhner. A fast, p-Multigrid Discontinuous Galerkin Method for Compressible Flows at All Speeds. AIAA journal, 46(3):635-652, 2008. Cited on page(s) 7, 42, 44.

[133] T. B. Lynn, D. W. Bechert, and D. A. Gerich. Direct drag measurements in a turbulent flat-plate boundary layer with turbulence manipulators. Experiments in Fluids, 19(6):405-416, 1995. Cited on page(s) 153.

[134] J. Manzanero, E. Ferrer, G. Rubio, and E. Valero. On the role of numerical dissipation in stabilising under-resolved turbulent simulations using discontinuous Galerkin methods. Journal of Computational Physics, 2018. Cited on page(s) 5 .

[135] J. Manzanero, G. Rubio, E. Ferrer, E. Valero, and D. A. Kopriva. Insights on Aliasing Driven Instabilities for Advection Equations with Application to Gauss-Lobatto Discontinuous Galerkin Methods. Journal of Scientific Computing, 75(3):1262-1281, 2018. Cited on page(s) 5, 74.

[136] J. Manzanero, G. Rubio, D. A. Kopriva, E. Ferrer, and E. Valero. A freeenergy stable nodal discontinuous Galerkin approximation with summationby-parts property for the Cahn-Hilliard equation. arXiv Numerical Analysis, 2019. Cited on page(s) 5, 74. 
[137] J. Manzanero, G. Rubio, D. A. Kopriva, E. Ferrer, and E. Valero. Entropystable discontinuous Galerkin approximation with summation-by-parts property for the incompressible Navier-Stokes equations with variable density and artificial compressibility. 2019. Cited on page(s) 74.

[138] J. Manzanero, A. M. Rueda-Ramírez, G. Rubio, and E. Ferrer. The Bassi Rebay 1 scheme is a special case of the Symmetric Interior Penalty formulation for discontinuous Galerkin discretisations with Gauss-Lobatto points. Journal of Computational Physics, 363:1-10, 2018. Cited on page(s) 25, 74.

[139] S. Marras, J. F. Kelly, M. Moragues, A. Müller, M. A. Kopera, M. Vázquez, F. X. Giraldo, G. Houzeaux, and O. Jorba. A Review of Element-Based Galerkin Methods for Numerical Weather Prediction: Finite Elements, Spectral Elements, and Discontinuous Galerkin. Archives of Computational Methods in Engineering, 23(4):673-722, 2016. Cited on page(s) 1, 5.

[140] R. Martin and H. Guillard. A second order defect correction scheme for unsteady problems. Computers and Fluids, 25(1):9-27, 1996. Cited on page(s) 98, 99.

[141] C. Mavriplis. Nonconforming Discretizations and a Posteriori Error Estimators for Adaptive Spectral Element Techniques. PhD thesis, Massachusetts Institute of Technology, 1989. Cited on page(s) 98, 100, 110.

[142] C. Mavriplis. Adaptive mesh strategies for the spectral element method. Computer methods in applied mechanics and engineering, 116(1-4):77-86, 1994. Cited on page(s) 98, 100.

[143] D. J. MAVRIPLIS. Accurate multigrid solution of the Euler equations on unstructured and adaptive meshes. AIAA Journal, 28(2):213-221, 1990. Cited on page(s) 3 .

[144] D. J. Mavriplis. An Assessment of Linear Versus Nonlinear Multigrid Methods for Unstructured Mesh Solvers. Journal of Computational Physics, 175(1):302-325, 2002. Cited on page(s) 42, 44.

[145] C. Mettot, F. Renac, and D. Sipp. Computation of eigenvalue sensitivity to base flow modifications in a discrete framework: Application to open-loop control. Journal of Computational Physics, 269:234-258, 2014. Cited on page(s) 60.

[146] H. W. Meuer. The TOP500 project: Looking back over 15 years of supercomputing experience. Informatik-Spektrum, 31(3):203-222, 2008. Cited on page(s) 2 . 
[147] A. Mignone, G. Bodo, S. Massaglia, T. Matsakos, O. Tesileanu, C. Zanni, and A. Ferrari. PLUTO: a Numerical Code for Computational Astrophysics. The Astrophysical Journal Supplement Series, 170:228-242, 2007. Cited on page(s) 1, 5 .

[148] C. A. A. Minoli and D. A. Kopriva. Discontinuous Galerkin spectral element approximations on moving meshes. Journal of Computational Physics, 230(5):1876-1902, 2011. Cited on page(s) 4, 5, 104.

[149] W. F. Mitchell and C. S. Division. The hp -Multigrid Method Applied to hp -Adaptive Refinement of Triangular Grids. Numerical Linear Algebra with Applications, 17(1):211-228, 2010. Cited on page(s) 7, 42, 44.

[150] F. Naddei, M. De La LLave Plata, V. Couaillier, and F. Coquel. A comparison of refinement indicators for the p-adaptive simulation of steady and unsteady flows with discontinuous Galerkin methods. Journal of Computational Physics, 376(1 January 2019):508-533, 2018. Cited on page(s) 99, 100, 173, 175.

[151] C. R. Nastase and D. J. Mavriplis. High-order discontinuous Galerkin methods using an hp-multigrid approach. Journal of Computational Physics, 213:330-357, 2006. Cited on page(s) 7, 42, 44, 45.

[152] N. C. Nguyen, J. Peraire, and B. Cockburn. An implicit high-order hybridizable discontinuous Galerkin method for nonlinear convection-diffusion equations. Journal of Computational Physics, 228(23):8841-8855, 2009. Cited on page(s) 75, 79.

[153] W. L. Oberkampf and C. J. Roy. Verification and validation in scientific computing. Cambridge University Press, 2010. Cited on page(s) 98.

[154] W. Pazner and P. O. Persson. Approximate tensor-product preconditioners for very high order discontinuous Galerkin methods. Journal of Computational Physics, 354:344-369, 2018. Cited on page(s) 93.

[155] J. Peraire, N.-C. Nguyen, and B. Cockburn. A Hybridizable Discontinuous Galerkin Method for the Compressible Euler and Navier-Stokes Equations. 20th AIAA Computational Fluid Dynamics Conference, page 3228, 2011. Cited on page(s) 75, 79.

[156] P.-O. Persson and J. Peraire. Sub-Cell Shock Capturing for Discontinuous Galerkin Methods. 44th AIAA Aerospace Sciences Meeting and Exhibit, pages 1-13, 2006. Cited on page(s) 99, 100.

[157] P.-O. Persson and J. Peraire. Newton-GMRES Preconditioning for Discontinuous Galerkin Discretizations of the Navier-Stokes Equations. SIAM Journal on Scientific Computing, 30(6):2709-2733, 2008. Cited on page(s) 92. 
[158] S. Petersen, C. Farhat, and T. Radek. A space-time discontinuous Galerkin method for the solution of the wave equation in the time domain Steffen. International Journal for Numerical Methods in Engineering, 78:275-295, 2009. Cited on page(s) 78.

[159] T. S. Phillips and C. J. Roy. Residual Methods for Discretization Error Estimation. 20th AIAA Computational Fluid Dynamics Conference, 3870(2730):665, 2011. Cited on page(s) 99, 103.

[160] N. A. Pierce and M. B. Giles. Adjoint Recovery of Superconvergent Functionals from PDE Approximations. SIAM Review, 42(2):247-264, 2003. Cited on page(s) 99.

[161] N. A. Pierce and M. B. Giles. Adjoint and defect error bounding and correction for functional estimates. Journal of Computational Physics, 200(2):769794, 2004. Cited on page(s) 99.

[162] S. Popinet. Gerris: A tree-based adaptive solver for the incompressible Euler equations in complex geometries. Journal of Computational Physics, 190(2):572-600, 2003. Cited on page(s) xxiii, 158.

[163] A. Pothen, A. H. Gebremedhin, and F. Manne. What Color Is Your Jacobian? Graph Coloring for Computing Derivatives. SIAM Review, 47(4):629, 2005. Cited on page(s) 58 .

[164] M. Raschi, F. Mut, G. Byrne, C. M. PInternational journal for numerical methods in biomedical engineeringutman, S. Tateshima, F. Vi $\{\backslash \sim \mathrm{n}\}$ uela, T. Tanoue, K. Tanishita, and J. R. Cebral. CFD and PIV analysis of hemodynamics in a growing intracranial aneurysm. International journal for numerical methods in biomedical engineering, 28(2):214-225, 2012. Cited on page(s) 1.

[165] P. Rasetarinera and M. Hussaini. An Efficient Implicit Discontinuous Spectral Galerkin Method. Journal of Computational Physics, 172(2):718-738, 2001. Cited on page(s) 4, 5 .

[166] W. H. Reed and T. R. Hill. Triangular mesh methods for the neutron transport equation. Triangular mesh methods for the neutron transport equation, Technical(LA-UR-73-479):1-23, 1973. Cited on page(s) 2, 3.

[167] J.-F. Remacle, J. E. Flaherty, and M. S. Shephard. An Adaptive Discontinuous Galerkin Technique with an Orthogonal Basis Applied to Compressible Flow Problems. Society for Industrial and Applied Mathematics. SIAM Review, 45(1):53-72, 2003. Cited on page(s) 99. 
[168] B. Rivière. Discontinuous Galerkin Methods for Solving Elliptic and Parabolic Equations Theory and Implementation. SIAM, 2008. Cited on page(s) 4, 7, 25.

[169] P. J. Roache. Verification and validation in computational science and engineering, volume 895. Hermosa Albuquerque, NM, 1998. Cited on page(s) 98, 99.

[170] P. L. Roe. Approximate Riemann solvers, parameter vectors, and difference schemes. Journal of Computational Physics, 43(2):357-372, 1981. Cited on page(s) 23, 24, 181.

[171] P. L. Roe and J. Pike. Efficient construction and utilisation of approximate Riemann solutions. In Proc. of the sixth int'l. symposium on Computing methods in applied sciences and engineering, VI, pages 499-518. North-Holland Publishing Co., 1985. Cited on page(s) 23, 24.

[172] J. Romero and A. Jameson. Extension of the Flux Reconstruction Method to Triangular Elements using Collapsed-Edge Quadrilaterals. In 54th AIAA Aerospace Sciences Meeting, page 1825, 2016. Cited on page(s) 165.

[173] E. M. Rønquist and A. T. Patera. Spectral element multigrid. I. Formulation and numerical results. Journal of Scientific Computing, 2(4):389-406, 1987. Cited on page(s) 41, 44, 53, 92.

[174] C. Roy. Review of Discretization Error Estimators in Scientific Computing. In 48th AIAA Aerospace Sciences Meeting Including the New Horizons Forum and Aerospace Exposition, Reston, Virigina, jan 2010. American Institute of Aeronautics and Astronautics. Cited on page(s) 98, 100.

[175] G. Rubio, F. Fraysse, J. De Vicente, and E. Valero. The estimation of truncation error by $\tau$-estimation for Chebyshev spectral collocation method. Journal of Scientific Computing, 57(1):146-173, 2013. Cited on page(s) 101, 114.

[176] G. Rubio, F. Fraysse, D. A. Kopriva, and E. Valero. Quasi-a priori truncation error estimation in the DGSEM. Journal of Scientific Computing, 64(2):425455,2015 . Cited on page(s) $97,100,101,102,103,105,106,107,108$, $114,119,122,146,149,168,169$.

[177] G. Rubio Calzado. Truncation error estimation in the Discontinuous Galerkin Spectral Element Method. PhD thesis, Universidad Politécnica de Madrid. School of Aeronautics (ETSIAE), 2015. Cited on page(s) 97, 101, 102, 105, 106, 107, 114, 119, 122. 
[178] A. M. Rueda-Ramírez, J. Manzanero, E. Ferrer, G. Rubio, and E. Valero. A pmultigrid strategy with anisotropic p-adaptation based on truncation errors for high-order discontinuous Galerkin methods. Journal of Computational Physics, 378:209-233, 2019. Cited on page(s) 8, 143, 168, 169.

[179] A. M. Rueda-Ramírez, G. Rubio, E. Ferrer, and E. Valero. An Anisotropic p-Adaptation Multigrid Scheme for the Discontinuous Galerkin Spectral Element Method. Lecture Notes in Computational Science and Engineering. Proceedings of the International Conference on Spectral and High-Order Methods (ICOSAHOM 2018). London, UK., 2019. Cited on page(s) 143.

[180] A. M. Rueda-Ramírez, G. Rubio, E. Ferrer, and E. Valero. Truncation Error Estimation in the p-Anisotropic Discontinuous Galerkin Spectral Element Method. Journal of Scientific Computing, 78(1):433-466, 2019. Cited on page(s) 5, 8, 101, 114, 168, 169.

[181] C. L. Rumsey, M. D. Sanetrik, R. T. Biedron, N. D. Melson, and E. B. Parlette. Efficiency and accuracy of time-accurate turbulent. AIAA Paper, page 1835, 1995. Cited on page(s) 172.

[182] Y. Saad. Iterative methods for sparse linear systems. SIAM, 2003. Cited on page(s) 46, 47, 49, 51, 91.

[183] D. Schwamborn, T. Gerhold, and R. Heinrich. The dlr tau-code: recent applications in research and industry. Eccomas, pages 1-25, 2006. Cited on page(s) 1,5 .

[184] K. Shahbazi. An explicit expression for the penalty parameter of the interior penalty method. Journal of Computational Physics, 205(2):401-407, 2005. Cited on page(s) 25.

[185] K. Shahbazi, D. J. Mavriplis, and N. K. Burgess. Multigrid algorithms for high-order discontinuous Galerkin discretizations of the compressible Navier-Stokes equations. Journal of Computational Physics, 228(21):79177940, 2009. Cited on page(s) 7, 42, 44, 45.

[186] J. Shen. Pseudo-compressibility methods for the unsteady incompressible navier-stokes equations. In Proceedings of the 1994 Beijing symposium on nonlinear evolution equations and infinite dynamical systems, pages 68-78, 1997. Cited on page(s) 203.

[187] S. Sherwin and G. Karniadakis. A triangular spectral element method; applications to the incompressible Navier-Stokes equations. Computer Methods in Applied Mechanics and Engineering, 123(1-4):189-229, 1995. Cited on page(s) 165. 
[188] S. J. Sherwin, R. M. Kirby, J. Peiró, R. L. Taylor, and O. C. Zienkiewicz. On 2D elliptic discontinuous Galerkin methods. International Journal for Numerical Methods in Engineering, 65(5):752-784, 2006. Cited on page(s) 75, 76, 77, 78, 90 .

[189] S. Soon, B. Cockburn, and H. K. Stolarski. A hybridizable discontinuous Galerkin method for linear elasticity. International Journal for Numerical Methods in Engineering, 80:1058-1092, 2009. Cited on page(s) 78.

[190] P. R. Spalart and V. Venkatakrishnan. On the role and challenges of CFD in the aerospace industry. Aeronautical Journal, 120(1223):209-232, 2016. Cited on page(s) 2 .

[191] A. Syrakos, G. Efthimiou, J. G. Bartzis, and A. Goulas. Numerical experiments on the efficiency of local grid refinement based on truncation error estimates. Journal of Computational Physics, 231(20):6725-6753, 2012. Cited on page(s) 100, 101, 144.

[192] A. Syrakos and A. Goulas. Finite volume adaptive solutions using SIMPLE as smoother. International Journal for Numerical Methods in Fluids, 52(11):1215-1245, 2006. Cited on page(s) 100, 101.

[193] E. F. Toro. Riemann solvers and numerical methods for fluid dynamics: a practical introduction. Springer Science \& Business Media, 2013. Cited on page(s) 3, 4, 23, 64 .

[194] L. N. Trefethen and D. Bau III. Numerical linear algebra, volume 50. Siam, 1997. Cited on page(s) 47.

[195] S. Turek and A. Ouazzi. Unified edge-oriented stabilization of nonconforming FEM for incompressible flow problems: Numerical investigations. Journal of Numerical Mathematics, 15(4):299-322, 2007. Cited on page(s) 3.

[196] D. A. Venditti and D. L. Darmofal. Grid Adaptation for Functional Outputs: Application to Two-Dimensional Inviscid Flows. Journal of Computational Physics, 176(1):40-69, 2002. Cited on page(s) 99.

[197] F. Verbosio, A. D. Coninck, D. Kourounis, and O. Schenk. Enhancing the scalability of selected inversion factorization algorithms in genomic prediction. Journal of Computational Science, 22(Supplement C):99-108, 2017. Cited on page(s) 47.

[198] H. K. Versteeg and W. Malalasekera. An introduction to computational fluid dynamics: the finite volume method. Pearson Education, 2007. Cited on page(s) 7,41 . 
[199] P. E. J. Vos, S. J. Sherwin, and R. M. Kirby. From h to p efficiently: Implementing finite and spectral/hp element methods to achieve optimal performance for low- and high-order discretisations. Journal of Computational Physics, 229(13):5161-5181, 2010. Cited on page(s) 4, 75, 76, 77.

[200] L. Wang and D. Mavriplis. Adjoint-based h-p Adaptive Discontinuous Galerkin Methods for the Compressible Euler Equations. Journal of Computational Physics, 228(20):7643-7661, 2009. Cited on page(s) 7, 42, $44,98,99,129$.

[201] L. Wang and D. J. Mavriplis. Implicit solution of the unsteady Euler equations for high-order accurate discontinuous Galerkin discretizations. Journal of Computational Physics, 225(2):1994-2015, 2007. Cited on page(s) 7, 42.

[202] Z. Wang, K. Fidkowski, R. Abgrall, F. Bassi, D. Caraeni, A. Cary, H. Deconinck, R. Hartmann, K. Hillewaert, H. Huynh, N. Kroll, G. May, P.-O. Persson, B. van Leer, and M. Visbal. High-order CFD methods: current status and perspective. International Journal for Numerical Methods in Fluids, 72(8):811-845, 2013. Cited on page(s) 2, 4, 6 .

[203] J. H. Williamson. Low-storage Runge-Kutta schemes. Journal of Computational Physics, 35(1):48-56, 1980. Cited on page(s) 33, 87.

[204] A. R. Winters, R. C. Moura, G. Mengaldo, G. J. Gassner, S. Walch, J. Peiro, and S. J. Sherwin. A comparative study on polynomial dealiasing and split form discontinuous Galerkin schemes for under-resolved turbulence computations. Journal of Computational Physics, 372:1-21, 2018. Cited on page(s) 5,75 .

[205] S. H. Yina. on Structur nconditi ionally Stable Explicit Method for Stru uctural An Un Dyna amics. In The Twelfth East Assia-Pacific Conference on Structural Engineering and Construction, volume 14, pages 2519-2526, 2011. Cited on page(s) 32 .

[206] T. A. Zang, Y. S. Wong, and M. Y. Hussaini. Spectral multigrid methods for elliptic equations II. Journal of Computational Physics, 54(3):489-507, 1984. Cited on page(s) 41.

[207] O. C. Zienkiewicz. The Finite Element Method. McGraw-hill, London, 2000. Cited on page(s) 3.

[208] O. C. Zienkiewicz. The background of error estimation and adaptivity in finite element computations. Computer Methods in Applied Mechanics and Engineering, 195(4-6):207-213, 2006. Cited on page(s) 98. 


